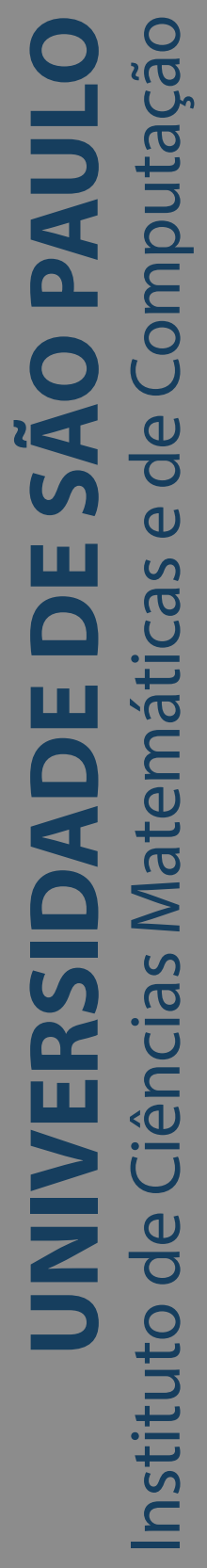

Models and algorithms for high school timetabling problems

\title{
Landir Saviniec
}

Tese de Doutorado do Programa de Pós-Graduação em Ciências de Computação e Matemática Computacional (PPG-CCMC) 

Data de Depósito:

Assinatura:

\section{Landir Saviniec}

\section{Models and algorithms for high school timetabling problems}

Doctoral dissertation submitted to the Institute of Mathematics and Computer Sciences - ICMC-USP, in partial fulfillment of the requirements for the degree of the Doctorate Program in Computer Science and Computational Mathematics. FINAL VERSION

Concentration Area: Computer Science and Computational Mathematics

Advisor: Prof. Dra. Maristela Oliveira dos Santos

Co-advisor: Prof. Dr. Alysson Machado Costa

\section{USP - São Carlos}

January 2018 
Ficha catalográfica elaborada pela Biblioteca Prof. Achille Bassi e Seção Técnica de Informática, ICMC/USP, com os dados inseridos pelo(a) autor(a)

Saviniec, Landir

S267m Models and algorithms for high school

timetabling problems / Landir Saviniec; orientador

Maristela Oliveira Santos; coorientador Alysson

Machado Costa. -- São Carlos, 2018.

$157 \mathrm{p}$.

Tese (Doutorado - Programa de Pós-Graduação em Ciências de Computação e Matemática Computacional) Instituto de Ciências Matemáticas e de Computação, Universidade de São Paulo, 2018.

1. Problema de horários educacionais. 2 . Programação linear inteira mista. 3. Metaheurísticas paralelas. 4. Geração de colunas. I. Santos, Maristela Oliveira, orient. II. Costa, Alysson Machado, coorient. III. Título.

Bibliotecários responsáveis pela estrutura de catalogação da publicação de acordo com a AACR2: Gláucia Maria Saia Cristianini - CRB - 8/4938

Juliana de Souza Moraes - CRB - 8/6176 


\section{Landir Saviniec}

\section{Modelos e algoritmos para problemas de horários escolares}

Tese apresentada ao Instituto de Ciências Matemáticas e de Computação - ICMC-USP, como parte dos requisitos para obtenção do título de Doutor em Ciências - Ciências de Computação e Matemática Computacional. VERSÃO REVISADA

Área de Concentração: Ciências de Computação e Matemática Computacional

Orientador: Prof. Dra. Maristela Oliveira dos Santos

Coorientador: Prof. Dr. Alysson Machado Costa 

I would like to thank all people who directly or indirectly contributed to this thesis. First, I thank God for giving me health and energy to finish this PhD course. Second, I thank Professors Maristela Oliveira dos Santos and Alysson Machado Costa for advising me, and my family for supporting me during this journey.

I also appreciate all attention and help from people who received me in the School of Mathematics and Statistics at The University of Melbourne, where I developed part of this thesis. Moreover, I would like to thank friends and colleagues from the University of São Paulo, especially those from the Optimization Laboratory, who used to share their coding and writing experience with me.

I am also grateful to Professor George H. G. Fonseca and his research group for sharing the code of their solver GOAL, which I used for comparisons in this thesis.

Lastly, I would like to thank FAPESP (Fundação de Amparo à Pesquisa do Estado de São Paulo) which funded this research through the grant numbers 2013/13563-3 and BEPE 2015/10032-2. 



\section{ABSTRACT}

SAVINIEC, L. Models and algorithms for high school timetabling problems. 2018. 157 p. Tese (Doutorado em Ciências - Ciências de Computação e Matemática Computacional) Instituto de Ciências Matemáticas e de Computação, Universidade de São Paulo, São Carlos SP, 2018.

High school timetabling problems consist in assigning meetings between classes and teachers, with the goal of minimizing the violation of specific soft requisites. This category of problems has been extensively studied since the 1950s, mostly via mixed-integer programming and metaheuristic techniques. However, the computation of optimal or near-optimal solutions using mixed-integer programs or metaheuristics is still a challenge for most practical problems. In this thesis, we investigate new mixed-integer programming formulations, column generation approaches and parallel metaheuristic based algorithms to compute lower bounds and solutions for high school timetabling problems. Extensive computational experiments conducted with real-world instances demonstrate that our best formulations are competitive with best-known formulations, while our parallel algorithms present superior performance than the state-of-the-art methods.

Keywords: High school timetabling problem, Mixed-integer programming, Column generation, Parallel metaheuristics. 



\section{RESUMO}

SAVINIEC, L. Modelos e algoritmos para problemas de horários escolares. 2018. 157 p. Tese (Doutorado em Ciências - Ciências de Computação e Matemática Computacional) Instituto de Ciências Matemáticas e de Computação, Universidade de São Paulo, São Carlos SP, 2018.

Problemas de horários escolares consistem em alocar encontros entre turmas e professores, com objetivo de minimizar violações a requisitos qualitativos específicos. Esta categoria de problemas tem sido largamente estudada desde 1950, particularmente via técnicas de programação linear inteira mista e metaheurísticas. Entretanto, a computação de soluções ótimas ou quase ótimas usando programas inteiro-mistos ou metaheurísticas ainda é um desafio na maioria dos problemas práticos. Nesta tese, nós investigamos novas formulações inteiro-mistas, decomposições por geração de colunas e algoritmos baseados em metaheurísticas paralelas para computar limitantes inferiores e soluções para problemas de horários escolares. Extensivos experimentos computacionais conduzidos com instâncias reais demonstram que nossas melhores formulações são competitivas com as melhores formulações existentes, enquanto nossos algoritmos paralelos são superiores em performance computacional quando comparados com métodos que são estado-da-arte.

Palavras-chave: Problema de horários escolares, Programação linear inteira mista, Geração de colunas, Metaheurísticas paralelas. 

Figure 1 - Class split. . . . . . . . . . . . . . . 37

Figure 2 - Class split with reshuffle. . . . . . . . . . . . 37

Figure 3 - Collaborative teaching. . . . . . . . . . . . 37

Figure $4-$ High school timetabling structure. . . . . . . . . . . . 46

Figure 5 - Parallel multi-start metaheuristic frameworks. . . . . . . . . . . . 9 90

Figure 6 - Best results for CMB with homogeneous ILS agents (4 threads). . . . . . . 94

Figure 7 - Best results for CMB with homogeneous TS and LAS agents (4 threads). . . 94

Figure 8 - Best results for CMB with homogeneous SA agents (4 threads). . . . . . . 95

Figure 9 - Best results for CMB with heterogeneous agents (4 threads). . . . . . . . 95

Figure 10 - Best results for DIMB parallel metaheuristics. . . . . . . . . . . . . 96

Figure 11 - Results of the best parallel algorithm DIMB-19I1D-8-0.05 compared to results of the best sequential ILS in all 34 instances from Table 15. . . . . . 97

Figure 12 - Results of the algorithm DIMB-19I1D-8-0.05 on problem HSTP-B with instances of Table 20. . . . . . . . . . . . . . . . . . . . 99

Figure 13 - Results of algorithm DIMB-19I1D-8-0.05 after 25 and 625 seconds compared to results of GOAL after 625 seconds on instances of Table 15. . . . . . . 101

Figure 14 - An illustration of the algorithm DIMB-CG. . . . . . . . . . . . . . . 104

Figure 15 - Timetable encodings. . . . . . . . . . . . . . . . 105

Figure 16 - Construction of solutions with information provided by columns. . . . . . . 106

Figure 17 - Preprocessing of variables belonging to class $c_{2}$ in subproblem $t_{4} \ldots \ldots$. . 109

Figure 18 - Proposed timetable encoding for the HSTP . . . . . . . . . . . . . . 133

Figure 19 - The TQ neighborhood generation with the proposed timetable encoding. . . 134

Figure 20 - Results of sequential TS for different parameter configurations. . . . . . . . 141

Figure 21 - Results of sequential SA for different parameter configurations. . . . . . . . 141

Figure 22 - Results of sequential LAS for different parameter configurations. . . . . . . 142

Figure 23 - Best configurations for sequential metaheuristics. . . . . . . . . . . . . 142 

Algorithm 1 - Pseudo-code of the CG procedure. . . . . . . . . . . . . . . . . 104

Algorithm 2 - Pseudo-code of the CCGH procedure . . . . . . . . . . . . 108

Algorithm 3 - Pseudo-code of the sequential ILS used by algorithm DIMB-CG. . . . . 109

Algorithm 4 - Pseudo-code of the diversifier procedure. . . . . . . . . . . . . . 110

Algorithm 5 - Pseudo-code of the manager procedure. . . . . . . . . . . . 111

Algorithm 6 - Pseudo-code of an intensifier procedure . . . . . . . . . . . . . . 112

Algorithm 7 - Pseudo-code of the constructive heuristic. . . . . . . . . . . . 135

Algorithm 8 - Pseudo-code of the sequential ILS . . . . . . . . . . . . . . . . . . . . . . . . . . . . .

Algorithm 9 - Pseudo-code of the sequential TS. . . . . . . . . . . . . . 137

Algorithm 10 - Pseudo-code of the sequential SA . . . . . . . . . . . 138

Algorithm 11 - Pseudo-code of the sequential LAS. . . . . . . . . . . . . 139 

Table 1 - Classification of university course timetabling requisites. . . . . . . . . . 33

Table 2 - List of examination timetabling requisites. . . . . . . . . . . . 35

Table 3 - List of high school timetabling requisites. . . . . . . . . . . . . . 40

Table 4 - Solution techniques for high school timetabling. . . . . . . . . . . . 43

Table 5 - Notation used in formulation CF1 . . . . . . . . . . . . . 50

Table 6 - An illustration of all possible assignment layouts in a day with four teaching periods. . . . . . . . . . . . . . . . . 56 56

Table 7 - Notation used in formulation CF2 . . . . . . . . . . . . 57

Table 8 - Notation used in formulation EF1. . . . . . . . . . . . . . . 64

Table 9 - Notation used in subproblem $\mathscr{P}_{r} \ldots \ldots \ldots \ldots$

Table 10 - Notation used in subproblem $\mathscr{P}_{t} \ldots \ldots \ldots$. . . . . . . . . . . . 67

Table 11 - Notation used in formulation EF2 . . . . . . . . . . . . . . . . 68

Table 12 - Notation used in subproblem $\mathscr{Q}_{t} \ldots \ldots \ldots$. . . . . . . . . . . . 69

Table 13 - Notation used in formulation EF3. . . . . . . . . . . . . . . . . 71

Table 14 - Notation used in subproblem $\mathscr{B}_{d} \ldots \ldots \ldots$. . . . . . . . . . . 72

Table 15 - Features of the 34 real instances from Brazilian high schools. . . . . . . . . . 74

Table 16 - Comparison of linear relaxations between formulations CF1 and CF2. . . . . 75

Table 17 - Results of the integer programs of formulations CF1 and CF2 in a time limit of three hours. . . . . . . . . . . . . . . . . . 77

Table 18 - The lower bounds of formulations EF1, EF2 and EF3 compared to lower bounds of formulation CF2-Cuts. . . . . . . . . . . . . . . . 79

Table 19 - Number of generated columns (NoC) in formulations EF1, EF2 and EF3. . . 80

Table 20 - Features of the instances proposed by Souza (2000). . . . . . . . . . . . . 81

Table 21 - The lower bounds generated by formulation EF2 on the instances of Souza (2000). . . . . . . . . . . . . . . . . . 81

Table 22 - Formulation CF2-Cuts compared to formulation CF-MCFP on problem HSTP-B. 82

Table 23 - Results of formulations CF2-Cuts $(\mathrm{TL}=3 \mathrm{~h})$ and EF2 on problem HSTP-C. . 84

Table 24 - Results of algorithm DIMB-19I1D-8-0.05 compared to results of formulation CF2 in all 34 instances of Table 15. . . . . . . . . . . . . . . . . . . 98

Table 25 - Results of algorithm DIMB-19I1D-8-0.05 compared to results of GOAL on problem HSTP-C with instances of Table 15. . . . . . . . . . . . . . . 100

Table 26 - List of parameter values used in the algorithm DIMB-CG. . . . . . . . . . 112

Table 27 - Comparison of convergence time (in seconds) between SCG and CG+CCGH. 113 
Table 28 - Number of columns generated by the SCG compared to CG+CCGH. . . . . . 114

Table 29 - Effectiveness of the two strategies used by the CCGH heuristic. . . . . . . 115

Table 30 - Quality of the CCGH solutions. . . . . . . . . . . . . . . 116

Table 31 - Results of the algorithm DIMB-CG compared to DIMB-19I1D-8-0.05 on problem HSTP-A. . . . . . . . . . . . . . . . . . 117

Table 32 - Results of the algorithm DIMB-CG compared to DIMB-19I1D-8-0.05 on problem HSTP-B. . . . . . . . . . . . . . . . . . . 118

Table 33 - Results of the algorithm DIMB-CG compared to DIMB-19I1D-8-0.05 on problem HSTP-C. . . . . . . . . . . . . . . . . . . . . 118 


\title{
LIST OF ABBREVIATIONS AND ACRONYMS
}

\author{
3-SAT 3-satisfiability problem \\ B\&B Branch-and-bound \\ BA Bee algorithm \\ C-ETP Capacitated examination timetabling problem \\ CB-CTP Curriculum-based course timetabling problem \\ CCG Cut and column generation \\ CCGH Constructive column generation heuristic \\ CCS Classes with compact schedules \\ CF-MCFP Compact formulation based on a multi-commodity flow problem \\ CF1 Compact formulation 1 \\ CF2 Compact formulation 2 \\ CF2-Cuts Formulation CF2 with the addition of Fenchel cuts \\ CG Column generation \\ CMB Central memory based algorithm \\ $\mathrm{CP} \quad$ Constraint programming \\ CSO Cat swarm optimization algorithm \\ CT Collaborative teaching \\ DIMB Diversification-intensification memory based algorithm \\ DIMB-CG DIMB algorithm with column generation \\ EB-CTP Post enrollment-based course timetabling problem \\ EF1 Extended formulation 1 \\ EF2 Extended formulation 2 \\ EF3 Extended formulation 3 \\ ETP Examination timetabling problem \\ F\&O Fix-and-optimize heuristic \\ GA Genetic algorithm \\ GHSTP Generalized high school timetabling problem \\ GOAL Heuristic solver of Fonseca et al. (2016) \\ GRASP Greedy randomized adaptive search procedure \\ HH Hyper-heuristics \\ HM Homogeneous algorithm
}


HSTP High school timetabling problem

HSTP-A The high school timetabling problem introduced by Saviniec (2013)

HSTP-B The high school timetabling problem introduced by Souza (2000)

HSTP-C A modified version of problem HSTP-B that is included in the ITC-2011

HT Heterogeneous algorithm

ILS Iterated local search

ITC-2002 First International Timetabling Competition

ITC-2007 Second International Timetabling Competition

ITC-2011 Third International Timetabling Competition

KHE Software library for high school timetabling by Jeff Kingston (KINGSTON, 2015)

LAS Late acceptance strategy

LB Lower bound

LP The optimal objective value of a linear program

LS-MaxSAT A local search algorithm with neighborhoods defined by maxSAT problems

MIP Mixed-integer programming

MISTA Multidisciplinary International Scheduling Conference: Theory \& Applications

NCCS No classes with compact schedules

NoC Number of columns

NRA No room allocation

NTPS No teachers preassigned to classes' subjects

PATAT International Conference on the Practice and Theory of Automated Timetabling

PB Population-based metaheuristics

PB-PI Parallel islands

PB-PIE Parallel individuals evaluation

RA Room allocation

SA Simulated annealing

STC Simultaneous temporary classes

TB Trajectory-based metaheuristics

TB-MA Move acceleration

TB-PM Parallel moves

TB-PMS Parallel multi-start

TL Time limit

TPS Teachers preassigned to classes' subjects

TQ Torque neighborhood operator

TS Tabu search

TSD-MIP Two-stage decomposition of an MIP model

U-ETP Uncapacitated examination timetabling problem 
UB Upper bound

UCTP University course timetabling problem

VNS Variable neighborhood search

XHSTT XML-based format to represent high school timetabling problems

XML Extensible markup language 



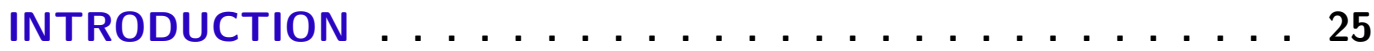

$1.1 \quad$ Research questions .................. 26

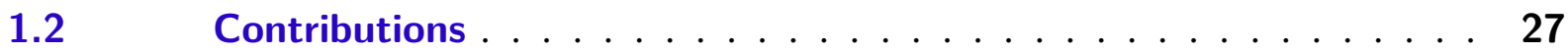

$1.3 \quad$ Thesis outline $\ldots \ldots \ldots \ldots \ldots \ldots$

2 EDUCATIONAL TIMETABLING AND PROBLEM DEFINITION . 29

$2.1 \quad$ University course timetabling problems . . . . . . . . . . 30

2.1.1 Curriculum-based course timetabling problems . . . . . . . . . . . 31

2.1.2 Post enrollment-based course timetabling problems . . . . . . . . . . 32

2.1.3 University course timetabling requisites . . . . . . . . . . . . . . . 33

2.2 Examination timetabling problems . . . . . . . . . . 33

2.2.1 Examination timetabling requisites . . . . . . . . . . . . 34

$2.3 \quad$ High school timetabling problems . . . . . . . . . . 35

2.3.1 High school timetabling requisites . . . . . . . . . . . . . 40

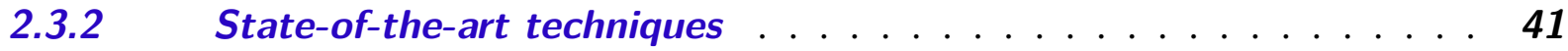

$2.4 \quad$ The problem under study . . . . . . . . . . . . . . 44

2.4.1 Definition ......................... 45

3 MIXED-INTEGER PROGRAMMING MODELS ........ 49

3.1 Compact formulations . . . . . . . . . . . . . . 49

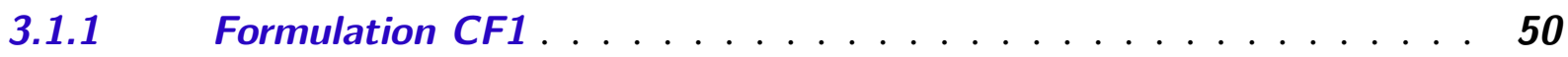

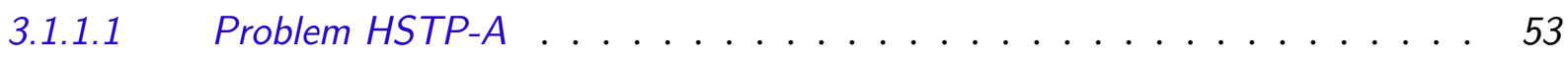

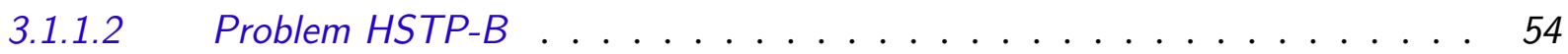

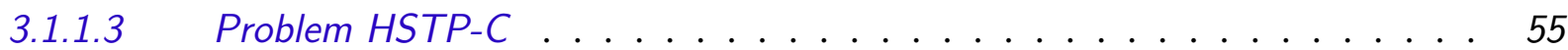

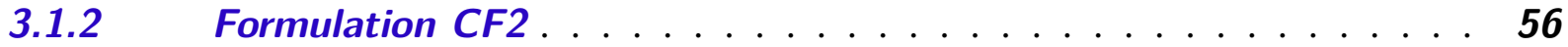

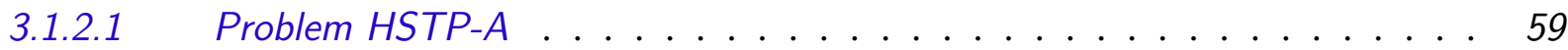

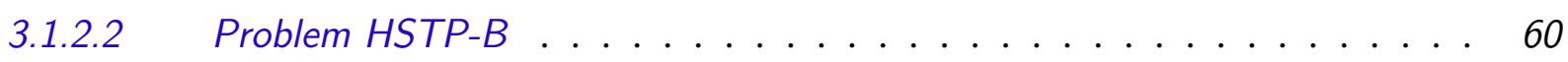

3.1.2.3 Problem HSTP-C . . . . . . . . . . . . . . . . 60

3.1.2.4 Cutting planes generation . . . . . . . . . . . . . 61

$3.2 \quad$ Column generation $\ldots \ldots \ldots \ldots \ldots$

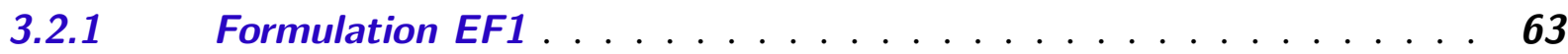

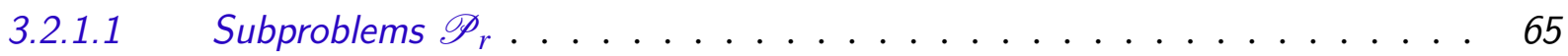

3.2.1.2 Subproblems $\mathscr{P}_{t} \ldots \ldots \ldots \ldots \ldots$ 


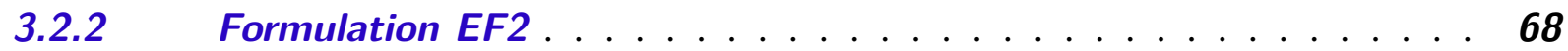

3.2.2.1 Subproblems $\mathscr{Q}_{t} \ldots \ldots \ldots \ldots$. . . . . . . . . . . . . 69

3.2.3 Formulation $E F 3 \ldots \ldots \ldots \ldots \ldots$

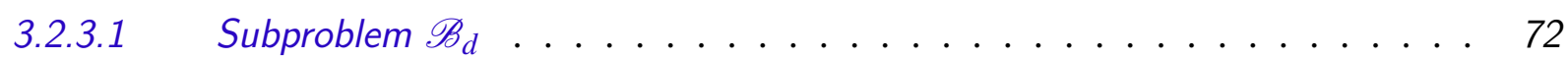

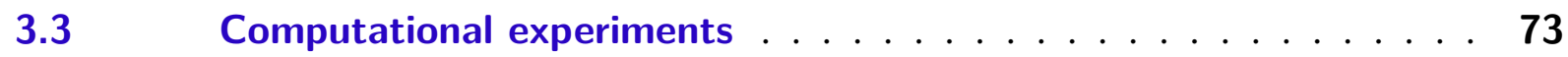

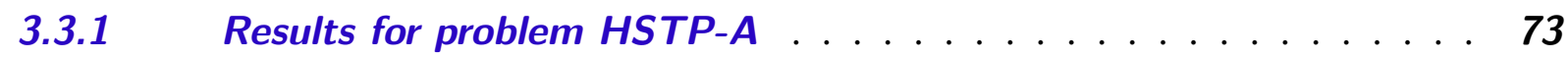

3.3.1.1 Comparing the linear programs of formulations CF1 and CF2 . . . . . . 74

3.3.1.2 Comparing the integer programs of formulations CF1 and CF2 . . . . . 75

3.3.1.3 Comparing formulation CF2 with EF1, EF2 and EF3 . . . . . . . 78

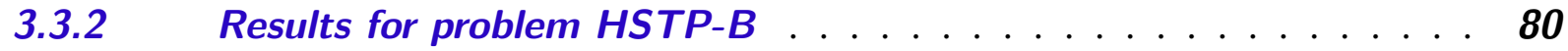

3.3.3 Results for problem HSTP-C . . . . . . . . . . . . . . 83

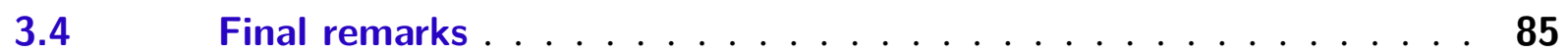

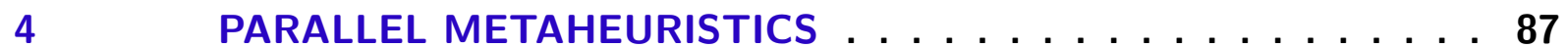

$4.1 \quad$ Existing parallel algorithms for high school timetabling . . . . . . 88

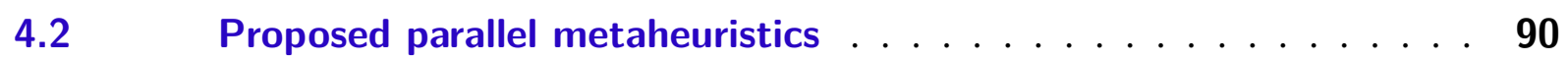

4.2.1 Central memory based algorithms . . . . . . . . . . . . 90

4.2.2 Diversification-intensification memory based algorithms . . . . . . 91

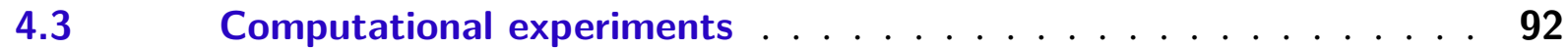

4.3.1 Comparisons among proposed methods . . . . . . . . . . 93

4.3.1.1 Results for central memory based algorithms . . . . . . . . . . . . . 93

4.3.1.2 Results for diversification-intensification memory based algorithms . . . . 96

4.3.1.3 Sequential versus parallel algorithms . . . . . . . . . . . . . . 97

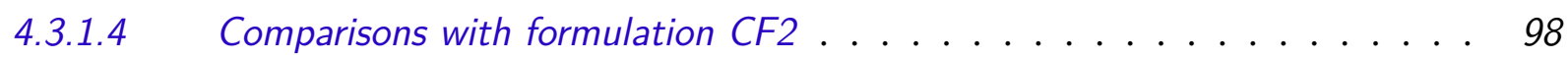

4.3.2 Comparisons with state-of-the-art algorithms . . . . . . . . 99

4.3.2.1 Comparisons with methods for problem HSTP-B . . . . . . . . . . . . 99

4.3.2.2 Comparisons with methods for problem HSTP-C . . . . . . . . . . . 100

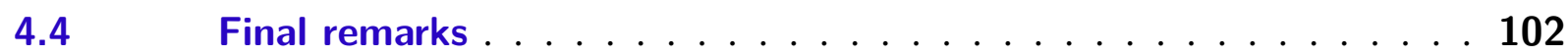

5 PARALLEL METAHEURISTIC WITH COLUMN GENERATION . . 103

$5.1 \quad$ A DIMB algorithm with column generation . . . . . . . 103

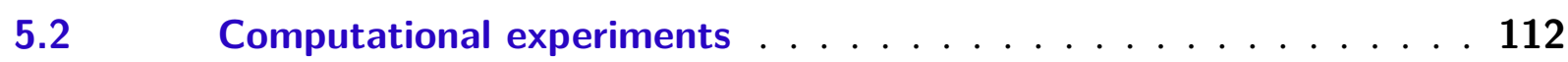

5.2.1 Analysis of the CCGH heuristic . . . . . . . . . . . 113

5.2.2 Analysis of the DIMB-CG algorithm . . . . . . . . . . 116

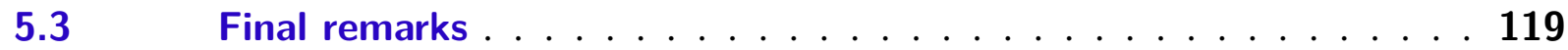

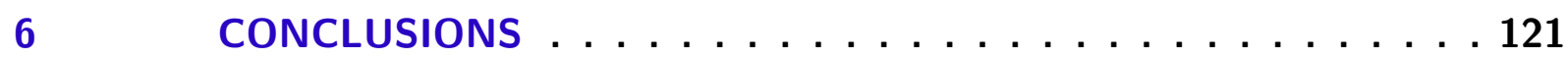

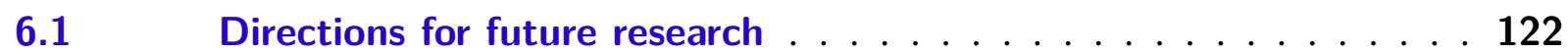


APPENDIX A DESCRIPTION AND TUNING OF THE STAND-ALONE METAHEURISTICS . . . . . . . . . . . . 133

A.1 Timetable encoding . . . . . . . . . . . . 133

A.2 Neighborhood structure . . . . . . . . . . . . . 134

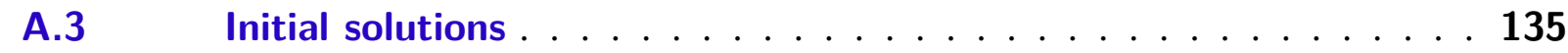

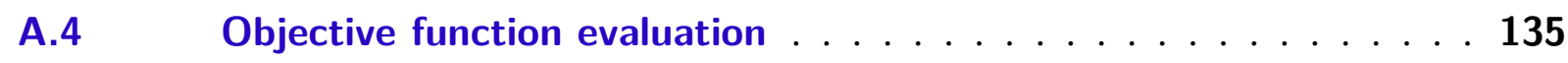

A.5 Sequential metaheuristics for high school timetabling . . . . . 136

A.5.1 Iterated local search . . . . . . . . . . . . . . . . 137

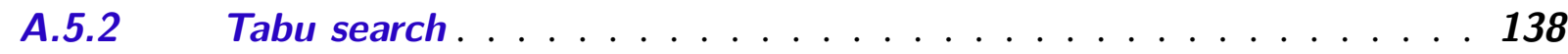

A.5.3 Simulated annealing . . . . . . . . . . . . . . 139

A.5.4 Late acceptance strategy . . . . . . . . . . . . . . . 139

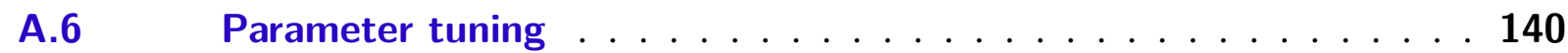

A.6.1 Results ..................... 140

APPENDIX B THE IMPROVED RELAX-AND-FIX HEURISTIC . . . 145 

CHAPTER

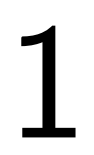

INTRODUCTION

Educational timetabling problems consist in scheduling encounters between teachers (or exams) and students. Specific situations give origin to different problems with a richness of characteristics. The scientific literature has branched this family of problems in three main categories: university course timetabling (LEWIS, 2008), examination timetabling (QU et al., 2009), and high school timetabling (WERRA, 1985; POST et al., 2012; PILLAY, 2014). Each of these categories has their set of specific constraints.

In this thesis, we address the high school timetabling problem (HSTP). Particularly, we focus on problems motivated by Brazilian high schools. A simplified decision version of the HSTP was shown to be NP-complete by polynomial reduction of the 3-SAT problem (EVEN; ITAI; SHAMIR, 1975). Instances originating from practical contexts naturally extend the requisites of this simplified problem. Extra requisites are usually associated with pedagogical preferences and social/cultural particularities. Examples of extra requisites are the need to assign double lessons (two consecutive lessons within the same day) for some classes' subjects (BIRBAS; DASKALAKI; HOUSOS, 2009; SANTOS et al., 2012), avoiding idle periods for teachers (periods during which the teacher is not busy, but is busy in earlier and later periods of the same day) (AVELLA et al., 2007), and the need to obtain compact schedules for teachers (SOUZA; MACULAN; OCHI, 2004; BIRBAS; DASKALAKI; HOUSOS, 2009).

Apart from its scientific importance, the high school timetabling is also of great significance in practice, because the school staff spend long hours to produce timetables that are usually of low quality or not acceptable. The automation of the timetabling process gives the schools more freedom to allocate their staff to other activities, and it produces timetables that offer more quality in the events scheduled for students and teachers.

Traditional compact mixed-integer programming (MIP) models (SOUZA, 2000; SANTOS et al., 2012; DORNELES; ARAÚJO; BURIOL, 2014; SøRENSEN; DAHMS, 2014; KRISTIANSEN; SØRENSEN; STIDSEN, 2015) for high school timetabling problems usually 
have weak linear programming relaxations, and the computation of optimal solutions for large size instances is still a challenge for actual black-box solvers. Given these limitations, many researchers have focused on combining heuristic algorithms and relaxation methods to obtain high-performance hybrid approaches and optimality bounds. On the one hand, research on heuristic algorithms (e.g., (TASSOPOULOS; BELIGIANNIS, 2012a; DORNELES; ARAÚJO; BURIOL, 2014; FONSECA et al., 2016; FONSECA; SANTOS; CARRANO, 2016; SAVINIEC; CONSTANTINO, 2017; SKOULLIS; TASSOPOULOS; BELIGIANNIS, 2017)) aims at the computation of high-quality solutions with reduced computational effort. On the other hand, the research on relaxation methods (e.g., decomposition by column generation (PAPOUTSIS; VALOUXIS; HOUSOS, 2003; SANTOS et al., 2012; DORNELES; ARAÚJO; BURIOL, 2017)) aims at the computation of tight lower bounds on the optimal solutions.

In this thesis, we address all the above topics. Section 1.1 enumerates our main research questions. Section 1.2 describes how we have contributed to push the boundaries of knowledge in each of these topics, and Section 1.3 presents an outline of the thesis.

\subsection{Research questions}

We have identified three main challenges for research in high school timetabling problems:

1. Improve compact mixed-integer programming formulations.

As mentioned before, traditional compact MIP models for high school timetabling problems are known to have weak linear relaxations, and therefore, their programs are still unable in providing good integer solutions within feasible computational time. Stronger formulations have been proposed (DORNELES; ARAÚJO; BURIOL, 2017; FONSECA et al., 2017). However, they still cannot effectively solve medium and large size instances.

2. Devise relaxation methods to compute tight lower bounds.

Strong lower bounds for high school timetabling problems can be generated by solving the linear relaxation of extended MIP formulations via column generation. In this case, such bounds can be compared with solutions found by heuristic algorithms to provide information on optimality gaps. Some extended formulations proposed to HSTP have shown strong lower bounds (PAPOUTSIS; VALOUXIS; HOUSOS, 2003; SANTOS et al., 2012; DORNELES; ARAÚJO; BURIOL, 2017), and recent heuristic approaches (FONSECA; SANTOS; CARRANO, 2016; SAVINIEC; CONSTANTINO, 2017; SKOULLIS; TASSOPOULOS; BELIGIANNIS, 2017) have found good quality solutions. However, optimality gaps for medium and large size instances are still not sufficiently small to conclude that state-of-the-art formulations and heuristics are effective for many practical instances. 
3. Devise parallel metaheuristic strategies that use the power of actual multiprocessor machines to exploit the search space better and improve the search quality.

Few strategies employing parallelism have been proposed and tested for educational timetabling problems. For high school timetabling problems, we found only three parallel approaches (ABRAMSON, 1991; ABRAMSON; ABELA, 1992; SRNDIC et al., 2009).

\subsection{Contributions}

In this thesis, we have investigated all research questions pointed above. Below, we describe our main findings on solution methods for high school timetabling problems, which are organized into five parts.

In the first contribution, we propose two MIP formulations which have a relatively small number of variables when applied to instances of practical problems. As we can explicitly generate all of their variables, such formulations will be denoted by compact models. The first formulation is based on traditional models and has a weak linear relaxation. The second formulation is based on the concept of "patterns", in which we enumerate all possible ways of assigning meetings to teaching periods within the days. This last formulation has a better linear relaxation, allowing for the generation of new cuts via the Fenchel enumeration cutting planes technique (BOYD, 1994a; BOYD, 1994b). In many instances, the additional cuts are sufficient to provide tight or even optimal lower bounds.

In the second contribution, we propose three alternative extended formulations for high school timetabling problems. As these formulations have a massive number of variables that are impracticable to generate explicitly, we resort to the column generation technique (DESAULNIERS; DESROSIERS; SOLOMON, 2005) to solve their linear relaxations. The optimal linear relaxation solution of such formulations provides a tight lower bound on the objective value of the optimal integer solutions.

In the third contribution, we propose parallel metaheuristic based algorithms. We investigate parallelization schemes in which different solution methods (agents) run concurrently in different processor threads. We analyze strategies such as:

Agents cooperation: the effect of allowing the agents to cooperate by exchanging their best solutions.

Diversification: the effect of using all agents as search intensification mechanisms or allowing at least one agent to diversify the search.

Agents diversity: all threads execute the same metaheuristic (homogeneous) or they execute different metaheuristics (heterogeneous).

In the fourth contribution, we propose a parallel metaheuristic with an embedded column generation. The method is composed of a column generation procedure, two constructive heuris- 
tics and a team of cooperative metaheuristics. While the column generation procedure computes a lower bound on the optimal solution of the problem, the constructive heuristics exploit the generated columns to build feasible solutions that are further improved by the metaheuristic threads. When the execution ends, the method provides a feasible integer solution and a lower bound on its optimal value.

In the last contribution, we propose a constructive heuristic for building initial feasible solutions. Our method is an improvement of the well-known MIP-based heuristic called relaxand-fix (DILLENBERGER et al., 1994). The proposed improvement is a strategy to avoid infeasible partitions, in which a proximity problem (FISCHETTI; MONACI, 2014) is solved to find an augmented partial solution, as soon as an infeasible partition is detected. This part is presented as an appendix of this thesis.

\subsection{Thesis outline}

The thesis is organized as follows. Chapter 2 gives an overview of educational timetabling problems, situating the high school timetabling problem among other problems of the same family. We present a detailed literature review of high school timetabling problems, classifying different problem variants and constraints, along with a discussion of the state-of-the-art techniques. The chapter closes with the definition of the problem to be studied throughout the thesis.

Chapter 3 is dedicated to mixed-integer programming techniques. We describe the two compact formulations, the generation of Fenchel cuts, and the three extended formulations which are solved by column generation. We close the chapter with an extensive set of experiments to evaluate the proposed formulations.

Chapter 4 describes our exploratory study that investigates the design of parallel metaheuristics for high school timetabling problems. In this chapter, we present an introduction to different parallelization schemes, review the existing parallel algorithms for high school timetabling problems and then, we propose several algorithm implementations based on two parallel frameworks. We close the chapter with a thorough numerical experimentation that compares our best parallel algorithm with state-of-the-art algorithms.

Chapter 5 describes the proposed parallel metaheuristic with embedded column generation. We outline the main ideas behind each part of this algorithm and provide experiments to assess its performance and the quality of the constructive heuristics.

Chapter 6 concludes the thesis with final remarks and directions for future research.

Finally, two appendices complete this thesis. Appendix A presents details on the standalone metaheuristics used by our parallel algorithms and their parameter setting procedures. The thesis ends with Appendix B, which describes the improved relax-and-fix heuristic. 
CHAPTER

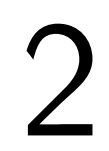

(1)

\section{EDUCATIONAL TIMETABLING AND PROBLEM DEFINITION}

Educational timetabling problems consist in assigning meetings between teachers (or exams) and students, considering a list of different requisites. Usually, these requisites are of two types: hard (mandatory) or soft (non-mandatory). A timetable that satisfies all hard requisites is said to be feasible, and a timetable that is feasible and also has a minimum number of violated soft requisites is said to be optimal.

In many educational organizations, the timetable construction is still made by hand, without the help of any computational tool. As frequent re-optimizations are necessary during the semester or year, the timetabling process usually consumes long working days.

The automated design of educational timetables started in the late 1950s, with methods that used to simulate manual procedures. Junginger (1986) presents a review of the first papers on timetabling problems, showing that simple heuristics were the most common solution methods. The article also reports on early computer programs that had been developed (GOTLIEB, 1963) using exact methods. However, they were able to address only small instances of simplified problems. Since then, timetabling problems have been extensively studied. The scientific literature has branched this family of problems in three main categories (SCHAERF, 1999b): university course timetabling problems (UCTP), examination timetabling problems (ETP), and high school timetabling problems (HSTP).

Educational timetabling problems have also been the subject of several international conferences and competitions. While the conferences aim at connecting researchers and promote new ideas, the competitions stimulate the field by encouraging researchers to share common datasets to validate their methods. Two international biennial conferences have been dedicated to educational timetabling, namely, the International Conference on the Practice and Theory of Automated Timetabling (PATAT) and the Multidisciplinary International Scheduling Conference: Theory \& Applications (MISTA). Also, the community of timetabling researchers have organized 
three international competitions:

- The First International Timetabling Competition (ITC-2002): this competition was dedicated to university course timetabling problems in 2002.

- The Second International Timetabling Competition (ITC-2007): this competition (<http:// www.cs.qub.ac.uk/itc2007/>) was dedicated to university course and examination timetabling problems in 2007 . The competition comprised three tracks:

- Track 1: it was dedicated to examination timetabling problems - there are 12 instances available.

- Track 2: it was dedicated to the post enrollment-based course timetabling problem, that is a variant of the UCTP - there are 24 instances available.

- Track 3: it was devoted to the curriculum-based course timetabling problem, that is another variant of the UCTP - there are 21 instances available.

- The Third International Timetabling Competition (ITC-2011): this competition ( $<$ https: //www.utwente.nl/ctit/hstt/itc2011/welcome/>) was dedicated to high school timetabling problems in 2011 (POST et al., 2016). There are 25 instances available (not including deprecated ones).

In the remainder of this chapter, we discuss each category of educational timetabling problems. In Sections 2.1 and 2.2, we present an overview of the key features of the university course and examination timetabling problems. In Section 2.3, we discuss the different variants of high school timetabling problems and review the state-of-the-art methods. We close the chapter in Section 2.4 with a definition of the problem studied in this thesis.

\subsection{University course timetabling problems}

The university course timetabling problem arises when the institution offers a set of courses, each course is taught several times a week, and students can enroll in their preferred courses. As some courses will have students in common, they must be scheduled to different periods, so that the students can attend all their courses (LEWIS et al., 2007; GASPERO; MCCOLLUM; SCHAERF, 2007; LEWIS, 2008). Furthermore, the use of space is another issue (e.g. capacity and availability) in this class of problems. Therefore, the use of classrooms must be optimized (SCHAERF, 1999b). Given these characteristics, the problem consists in assigning periods and classrooms to a set of courses, such that conflicting courses (courses with students in common) are scheduled to different periods.

We can also distinguish between two variants of university course timetabling problems (KRISTIANSEN; STIDSEN, 2013): curriculum-based course timetabling problems 
(CB-CTP) and post enrollment-based course timetabling problems (EB-CTP). In the former, conflicting courses are grouped by curriculum, while in the latter, conflicting courses are determined after students enrollment.

\subsubsection{Curriculum-based course timetabling problems}

In curriculum-based course timetabling problems, courses are grouped by curriculum. A curriculum is a group of courses that may have students in common. Therefore, all courses of a curriculum must be scheduled to different periods. Let us consider the following notations:

- $K$ : a set of courses.

- $G$ : a set of curricula.

- $H$ : a set of weekly periods.

- $l_{k}$ : the number of weekly lectures to be scheduled for course $k \in K$.

- $r_{h}$ : the number of classrooms available at period $h \in H$.

- $y_{k h}$ : binary decision variable which indicates whether course $k \in K$ is scheduled to period $h \in H$.

The basic curriculum-based course timetabling problem can be formulated as an integer linear programming model as follows (WERRA, 1985; SCHAERF, 1999b):

Find $y_{k h}$

Subject to:

$$
\begin{array}{ll}
\sum_{h \in H} y_{k h}=l_{k} & \forall k \in K \\
\sum_{k \in K} y_{k h} \leq r_{h} & \forall h \in H \\
\sum_{k \in \widehat{K}} y_{k h} \leq 1 & \forall h \in H ; \widehat{K} \in G \\
y_{k h} \in\{0,1\} & \forall k \in K ; h \in H
\end{array}
$$

In this formulation, constraints (2.2) ensure that the lectures of each course are scheduled. Constraints (2.3) avoid schedules in which there are more courses in a given period than the number of classrooms available. Lastly, constraints (2.4) prevent courses belonging to a curriculum $\widehat{K} \in G$ be scheduled to the same period.

This basic formulation can be used only for finding feasible solutions. Practical problems may involve a number of additional requisites. The reader is referred to the CB-CTP utilized in 
track 3 of the ITC-2007 for a more realistic problem (GASPERO; MCCOLLUM; SCHAERF, 2007).

\subsubsection{Post enrollment-based course timetabling problems}

In post enrollment-based course timetabling problems, there are no curricula, and conflicting courses are determined after the students have enrolled in their preferred courses. Therefore, any pair of conflicting courses must be scheduled to different periods. Let us consider the following notations:

- $K$ : a set of courses.

- $H$ : a set of weekly periods.

- $l_{k}$ : the number of weekly lectures to be scheduled for course $k \in K$.

- $r_{h}$ : the number of classrooms available at period $h \in H$.

- $C_{|K| \times|K|}$ : a matrix of conflicting courses, in which $c_{i j}$ is the number of students in common enrolled in both courses $i, j \in K$;

- $y_{k h}$ : binary decision variable which indicates whether course $k \in K$ is scheduled to period $h \in H$.

The basic post enrollment-based course timetabling problem can be formulated as an integer non-linear programming model as follows (PETROVIC; BURKE, 2004):

$$
\begin{aligned}
\text { Find } \quad y_{k h} & \\
\text { Subject to: } & \\
\sum_{h \in H} y_{k h}=l_{k} & \forall k \in K \\
\sum_{k \in K} y_{k h} \leq r_{h} & \forall h \in H \\
\sum_{i \in K} \sum_{j \in K} \sum_{h \in H} c_{i j} y_{i h} y_{j h}=0 & \\
y_{k h} \in\{0,1\} \quad & \forall k \in K ; h \in H
\end{aligned}
$$

This formulation is similar to formulation (2.1) - (2.5), except that constraints (2.4) are replaced by constraints (2.9), which state that every pair of conflicting courses cannot run simultaneously. The reader is referred to track 2 of the ITC-2007 for a practical EB-CTP (LEWIS et al., 2007). 


\subsubsection{University course timetabling requisites}

Practical situations may involve a number of other requisites. Bonutti et al. (2012) reviewed the literature and classified the main hard $(\mathrm{H})$ and soft $(\mathrm{S})$ requisites that may appear in university course timetabling problems. Their classification has two groups: the basic group formed by the most common requisites found when comparing a number of different problems, which constitutes the core of the UCTP, and the optional group - which is the group of unusual requisites. We summarize this classification in Table 1.

Table 1 - Classification of university course timetabling requisites.

\begin{tabular}{|c|c|c|}
\hline Requisite & Description & Type \\
\hline \multicolumn{3}{|c|}{ Basic requisites } \\
\hline Lectures & The lectures of all courses must be scheduled. & $\mathrm{H}$ \\
\hline Conflicts & $\begin{array}{l}\text { Courses that have the same teacher or students in common must be scheduled } \\
\text { to different periods. }\end{array}$ & $\mathrm{H}$ \\
\hline RoomOccupancy & Each classroom must host only one course per period. & $\mathrm{H}$ \\
\hline RoomCapacity & Classrooms must not be overloaded. & $\mathrm{H}$ \\
\hline Availability & Do not assign courses to periods in which their teachers are unavailable. & $\mathrm{H}$ \\
\hline \multicolumn{3}{|c|}{ Optional requisites } \\
\hline RoomStability & All lectures of a course should be scheduled to the same classroom. & S \\
\hline MinimumWorkingDays & $\begin{array}{l}\text { The lectures of each course should be spread over a minimum number of } \\
\text { days. }\end{array}$ & S \\
\hline ConsecutiveLectures & Courses of the same curriculum should be scheduled to consecutive periods. & S \\
\hline DoubleLessons & $\begin{array}{l}\text { Some courses may require some double lessons (two consecutive lessons } \\
\text { within the same day). }\end{array}$ & S \\
\hline StudentMinMaxLoad & $\begin{array}{l}\text { For each curriculum the number of daily lectures should be within a given } \\
\text { range ( } \min , \max ) \text {. }\end{array}$ & S \\
\hline RoomSuitability & $\begin{array}{l}\text { Course should be scheduled to classrooms that contain required equipments } \\
\text { (e.g. projectors, computers, etc). }\end{array}$ & $S$ \\
\hline TravelDistance & $\begin{array}{l}\text { Students should have enough time to move from one building to another } \\
\text { between two lectures. }\end{array}$ & $\mathrm{S}$ \\
\hline
\end{tabular}

The requisite "RoomCapacity" can also be soft, as in Gaspero, McCollum and Schaerf (2007).

\subsection{Examination timetabling problems}

The examination timetabling problem consists in assigning periods and classrooms to a set of exams so that students can attend all their required exams without clashes. Abdullah (2006) distinguishes between two variants of examination timetabling problems: capacitated examination timetabling problems (C-ETP), when classroom capacity is considered, and uncapacitated examination timetabling problems (U-ETP), when classroom capacity is not considered.

The examination timetabling problem can be formulated (SCHAERF, 1999b) similarly as made for the university course timetabling problem in the previous section. Let us consider the following notations: 
- $E$ : a set of exams that must be attended by students.

- $H$ : a set of periods (e.g. a planning horizon distributed over one or more weeks).

- $l_{h}$ : the maximum number of exams that can be scheduled at period $h \in H$.

- $G$ : a set of conflicting exam groups $\widehat{E}_{1}, \widehat{E}_{2}, \cdots$. In a conflicting exam group, all exams must be scheduled to different periods because they have students in common.

- $y_{e h}$ : binary decision variable which indicates whether exam $e \in E$ is scheduled to period $h \in H$.

The basic examination timetabling problem can be formulated as an integer linear programming model as follows (SCHAERF, 1999b):

Find $y_{e h}$

Subject to:

$$
\begin{array}{ll}
\sum_{h \in H} y_{e h}=1 & \forall e \in E \\
\sum_{e \in E} y_{e h} \leq l_{h} & \forall h \in H \\
\sum_{e \in \widehat{E}} y_{e h} \leq 1 & \forall h \in H ; \widehat{E} \in G \\
y_{e h} \in\{0,1\} & \forall e \in E ; h \in H
\end{array}
$$

In this formulation, constraints (2.12) ensure that all exams are scheduled. Constraints (2.13) ensure that no more than $l_{h}$ exams are scheduled for each period $h$. These last constraints are useful if it is required to place only one exam per classroom, and in each period $h$ there are only $l_{h}$ classrooms available. Lastly, constraints (2.14) prevent the exams belonging to the same conflicting group be scheduled to the same period.

Although the basic formulation of the ETP is similar to the basic formulation of the CBCTP presented in Section 2.1.1, practical problems usually include a number of other constraints that make the two problems different from each other. The reader is referred to the ETP used in track 1 of the ITC-2007 for a more detailed problem (MCCOLLUM et al., 2007).

\subsubsection{Examination timetabling requisites}

In this section, we list the most common examination timetabling requisites found in the literature. The list is shown in Table 2. This list is mainly based on Burke et al. (1996) and Gaspero and Schaerf (2001). Burke et al. (1996) surveyed the most common requisites considered in British universities through a questionnaire sent to 95 universities. However, they 
do not specify the type of each requisite, and we describe them as being hard or soft in Table 2 . Gaspero and Schaerf (2001) also describe some important hard requisites in their case study, as shown in Table 2.

Table 2 - List of examination timetabling requisites.

\begin{tabular}{|c|c|c|}
\hline Requisite & Description & Type \\
\hline \multicolumn{3}{|c|}{ Burke et al. (1996) } \\
\hline RoomCapacity & Classrooms must not be overloaded. & $\mathrm{H} / \mathrm{S}$ \\
\hline SimilarExams & Exams with questions in common must be scheduled to the same period. & $\mathrm{H} / \mathrm{S}$ \\
\hline Preference & Some exams may only be scheduled within a particular set of periods. & $\mathrm{H} / \mathrm{S}$ \\
\hline Preference & Only exams of the same duration should be placed into the same room. & $\mathrm{H} / \mathrm{S}$ \\
\hline Preference & $\begin{array}{l}\text { Exams that have the greatest number of students should be scheduled to } \\
\text { early periods. }\end{array}$ & $\mathrm{H} / \mathrm{S}$ \\
\hline RoomPreference & Some exams must be placed only into particular classrooms. & $\mathrm{H} / \mathrm{S}$ \\
\hline RoomPreference & Large exam halls must be scheduled in preference to smaller ones. & $\mathrm{H} / \mathrm{S}$ \\
\hline ExamPrecedence & Some exams must be scheduled before others. & $\mathrm{H} / \mathrm{S}$ \\
\hline ScatteredExams & No student is scheduled to attend exams in two consecutive periods. & $\mathrm{H} / \mathrm{S}$ \\
\hline ExamDailyLimit & No student is scheduled to more than one exam per day. & $\mathrm{H} / \mathrm{S}$ \\
\hline ScatteredExams & $\begin{array}{l}\text { Students should have their exams scattered as much as possible over the } \\
\text { planning horizon. }\end{array}$ & $\mathrm{H} / \mathrm{S}$ \\
\hline edExams & No student is scheduled to attend exams on two consecutive days. & $\mathrm{H} / \mathrm{S}$ \\
\hline Location & Exams must be scheduled to classrooms near their department. & $\mathrm{H} / \mathrm{S}$ \\
\hline \multicolumn{3}{|c|}{ Gaspero and Schaerf (2001) } \\
\hline Exams & All exams must be scheduled. & $\mathrm{H}$ \\
\hline Conflicts & Exams that have students in common must be scheduled to different periods. & $\mathrm{H}$ \\
\hline Capacity & $\begin{array}{l}\text { The number of students scheduled to attend exams at a given period must } \\
\text { not exceed the total number of available seats. }\end{array}$ & $\mathrm{H}$ \\
\hline Preassignments & Some exams may have to be necessarily scheduled to specific periods. & $\mathrm{H}$ \\
\hline Availability & Some exams may not be scheduled to some predefined periods. & $\mathrm{H}$ \\
\hline
\end{tabular}

\subsection{High school timetabling problems}

In high school timetabling problems, students are usually grouped by class. The classes are disjoint groups of students enrolled in the same list of subjects. Each subject has a number of weekly lessons that are usually taught by a preassigned teacher. Given these characteristics, the problem consists in assigning weekly periods to a set of class/teacher meetings, such that no class or teacher is required to attend more than one meeting per period.

The HSTP has several variants. Related to the use of classrooms, we can distinguish between:

- Problems without classroom allocation (NRA): in this variant, each class has its fixed classroom and therefore, there is no need for considering classroom allocations (SOUZA; MACULAN; OCHI, 2004; VALOUXIS; HOUSOS, 2003; SOUSA; MORETTI; PODESTÁ, 2008).

- Problems with classroom allocation (RA): in this variant, the schools do not have enough space to assign a fixed classroom for each class. Therefore, classrooms must be properly 
allocated during the timetable construction (JACOBSEN; BORTFELDT; GEHRING, 2006; WILKE; OSTLER, 2008; POST; AHMADI; GEERTSEMA, 2012).

Other two variants are related to the assignment of teachers to classes' subjects.

- Problems without teachers preassigned to classes' subjects (NTPS): in this variant, the assignment of teachers to each class' subject is made by the scheduling system during the timetable construction (ALVAREZ-VALDÉS; PARREÑO; TAMARIT, 2002).

- Problems with teachers preassigned to classes' subjects (TPS): in this variant, the assignment of teachers to each class' subject is previously made by the school staff (e.g. using trivial rules, such as priority due to seniority). Therefore, the scheduling system only needs to assign periods (or both, periods and classrooms) to meetings (SOUZA; MACULAN; OCHI, 2004; SOUSA; MORETTI; PODESTÁ, 2008).

We can also distinguish between:

- Problems in which classes do not have compact schedules (NCCS): the classes have free periods during the week, and usually, these free periods should be scheduled to the last periods of the days (JACOBSEN; BORTFELDT; GEHRING, 2006; AVELLA et al., 2007).

- Problems in which classes have compact schedules (CCS): the classes are busy in all periods during the week (SOUZA; MACULAN; OCHI, 2004; SOUSA; MORETTI; PODESTÁ, 2008).

Moreover, some variants may also involve the creation of temporary classes and collaborative teaching (BELIGIANNIS et al., 2008; BIRBAS; DASKALAKI; HOUSOS, 2009; TASSOPOULOS; BELIGIANNIS, 2012a).

- Simultaneous temporary classes (STC): during some periods, the students of one or more classes are re-organized into temporary classes, that must be simultaneously taught by different teachers. We may have two cases:

- Class split: a class $c$ may have a subject (e.g. computers, physics or technology) that requires lab work. If the lab room cannot fit all students, then two sub-classes $c_{i}$ and $c_{j}$ are formed. While the sub-class $c_{i}$ attends the mentioned subject with a teacher $t_{i}$, the other sub-class $c_{j}$ attends another subject with a teacher $t_{j}$, as illustrated in Figure 1. 


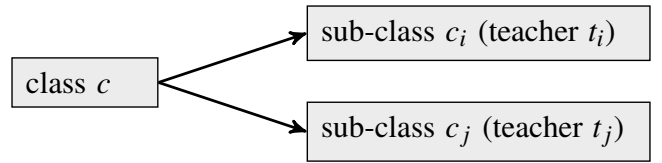

Figure 1 - Class split.

- Class split with reshuffle: some subjects, such as foreign languages, may have two levels, beginners and advanced. For example, supposing that there is only one teacher of English (say $t_{h}$ ), then two classes $c_{i}$ and $c_{j}$ might be split into sub-classes $c_{i 1}$, $c_{i 2}, c_{j 1}$ and $c_{j 2}$, so that the students in sub-classes $c_{i 1}$ and $c_{j 1}$ are joined together to form a new class (say $c_{h}$ ) to attend the beginners level, while the remaining students form another class (say $c_{k}$ ) and attend another subject (e.g. physical education), as illustrated in Figure 2.

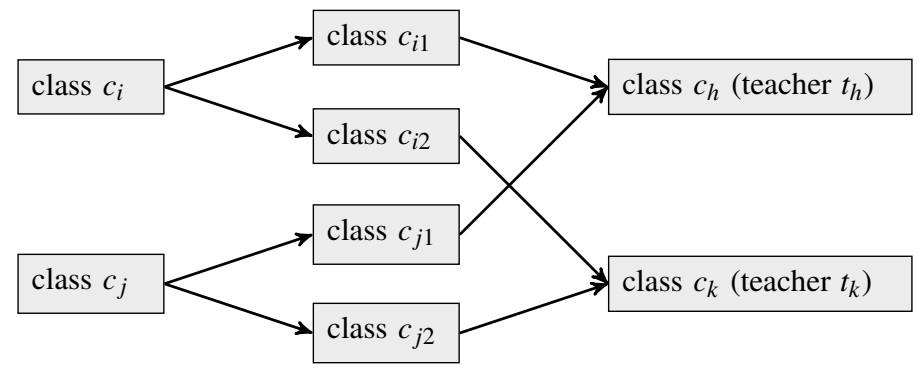

Figure 2 - Class split with reshuffle.

- Collaborative teaching (CT): some subjects may require lab lectures with two teachers working together to teach a large group of students, as illustrated in Figure 3.

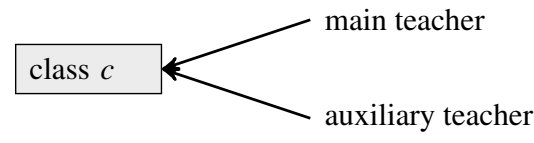

Figure 3 - Collaborative teaching.

Let us consider the following notations:

- $C$ : a set of classes.

- $T$ : a set of teachers.

- $D$ : a set of days (usually one week).

- $H$ : a set of teaching periods per day.

- $r_{c t}$ : the number of weekly lectures that class $c \in C$ must attend with teacher $t \in T$. 
- $x_{c t d h}$ : binary decision variable which indicates whether class $c \in C$ and teacher $t \in T$ meet at period $h \in H$ of day $d \in D$.

The basic high school timetabling problem can be formulated as an integer linear programming model as follows (WERRA, 1971; SCHAERF, 1999b):

\section{Find $x_{c t d h}$}

Subject to:

$$
\begin{aligned}
\sum_{d \in D} \sum_{h \in H} x_{c t d h}=r_{c t} & \forall c \in C ; t \in T \\
\sum_{t \in T} x_{c t d h} \leq 1 & \forall c \in C ; d \in D ; h \in H \\
\sum_{c \in C} x_{c t d h} \leq 1 & \forall t \in T ; d \in D ; h \in H \\
x_{c t d h} \in\{0,1\} & \forall c \in C ; t \in T ; d \in D ; h \in H
\end{aligned}
$$

In this formulation, constraints (2.17) ensure that all required lectures are scheduled, and constraints (2.18) and (2.19) impose that no class or teacher is required to attend more than one meeting per period. This fundamental problem can be solved in polynomial time by a min-cost network flow algorithm (WERRA, 1971).

Werra (1985) describes a formulation to deal with pre-assignment of lectures and unavailability of teachers and classes that is NP-Complete (EVEN; ITAI; SHAMIR, 1975). Let us consider the following parameters:

- $f_{c t d h}$ : binary constant that indicates whether class $c$ is pre-assigned to meet teacher $t$ at period $h$ of day $d$.

- $m_{c d h}$ : binary constant that indicates whether class $c$ is available at period $h$ of day $d$.

- $q_{t d h}$ : binary constant that indicates whether teacher $t$ is available at period $h$ of day $d$.

The problem with pre-assignments and unavailabilities can be formulated as: 
Find $x_{c t d h}$

Subject to:

$$
\begin{aligned}
\sum_{d \in D} \sum_{h \in H} x_{c t d h}=r_{c t} & \forall c \in C ; t \in T \\
\sum_{t \in T} x_{c t d h} \leq m_{c d h} & \forall c \in C ; d \in D ; h \in H \\
\sum_{c \in C} x_{c t d h} \leq q_{t d h} & \forall t \in T ; d \in D ; h \in H \\
x_{c t d h} \geq f_{c t d h} & \forall c \in C ; t \in T ; d \in D ; h \in H \\
x_{c t d h} \in\{0,1\} & \forall c \in C ; t \in T ; d \in D ; h \in H
\end{aligned}
$$

In this formulation, constraints (2.18) and (2.19) of the previous formulation are replaced by constraints (2.23) and (2.24) to ensure that classes and teachers are only scheduled when they are available. Also, pre-assigned lectures can be managed by considering the additional constraints $(2.25)$.

The two previous formulations are very limited in practice. Practical problems usually include a number of additional requisites that cannot be completely satisfied, because they conflict with each other. As an alternative, practical formulations seek for minimizing the number of times that conflicting requisites are violated. One of the first formulations in this direction is due to Junginger (1986). The author considers the minimization of lectures occurring in undesirable periods. The formulation is as follows:

$$
\begin{array}{cl}
\text { Minimize } & \sum_{c \in C} \sum_{t \in T} \sum_{d \in D} \sum_{h \in H} w_{c t d h} x_{c t d h} \\
\text { Subject to: } & \\
\sum_{d \in D} \sum_{h \in H} x_{c t d h}=r_{c t} & \forall c \in C ; t \in T \\
\sum_{t \in T} x_{c t d h} \leq m_{c d h} & \forall c \in C ; d \in D ; h \in H \\
\sum_{c \in C} x_{c t d h} \leq q_{t d h} & \forall t \in T ; d \in D ; h \in H \\
x_{c t d h} \in\{0,1\} & \forall c \in C ; t \in T ; d \in D ; h \in H
\end{array}
$$

In this formulation, $w_{c t d h}$ is a parameter that expresses the preference for assigning class $c$ to meet teacher $t$ at period $h$ of day $d$. The greater the value of $w_{c t d h}$, the smaller the preference for this assignment. 


\subsubsection{High school timetabling requisites}

Apart from the basic formulations described above, practical high school timetabling problems usually involve several other specific requisites. In Table 3, we classify the most common high school timetabling requisites found in the literature. An alternative classification is also available in Pillay (2014).

Table 3 - List of high school timetabling requisites.

\begin{tabular}{|c|c|c|}
\hline Requisite & Description & Type \\
\hline Lectures & All lectures must be scheduled (SCHAERF, 1999b). & $\mathrm{H}$ \\
\hline Conflicts & $\begin{array}{l}\text { No class or teacher is required to attend more than one meeting per period } \\
\text { (SCHAERF, 1999b). }\end{array}$ & $\mathrm{H}$ \\
\hline Availability & $\begin{array}{l}\text { No class or teacher is assigned to periods that they are unavailable (WERRA, } \\
1985 \text { ). }\end{array}$ & $\mathrm{H}$ \\
\hline RoomOccupancy & Each classroom must host only one lecture per period (ABRAMSON, 1991). & $\mathrm{H}$ \\
\hline Availability & $\begin{array}{l}\text { Classrooms cannot be used in periods that they are unavailable (JACOBSEN; } \\
\text { BORTFELDT; GEHRING, 2006; POST; AHMADI; GEERTSEMA, 2012). }\end{array}$ & $\mathrm{H}$ \\
\hline RoomCapacity & Classrooms must not be overloaded (WILKE; OSTLER, 2008). & $\mathrm{H}$ \\
\hline TemporaryClasses & Class splits and reshuffles must be satisfied, see Section 2.3 . & $\mathrm{H}$ \\
\hline CollaborativeTeaching & $\begin{array}{l}\text { Auxiliary teachers must be assigned to lectures that require collaborative } \\
\text { teaching, see Section } 2.3 \text {. }\end{array}$ & $\mathrm{H}$ \\
\hline WorkloadLimit & $\begin{array}{l}\text { Classes should not attend more than a given number of lectures of the same } \\
\text { subject per day (SOUZA; MACULAN; OCHI, 2004; AVELLA et al., 2007). }\end{array}$ & $\mathrm{H} / \mathrm{S}$ \\
\hline WorkloadLimit & $\begin{array}{l}\text { Classes that do not have compact schedules should have their daily work- } \\
\text { loads ranging between a (min, max) number of lectures (AVELLA et al., } \\
\text { 2007; MARTE, 2007). }\end{array}$ & $\mathrm{H} / \mathrm{S}$ \\
\hline WorkloadLimit & $\begin{array}{l}\text { Teachers should have their daily workloads ranging between a (min, max) } \\
\text { number of lectures (SCHAERF, 1999a; AVELLA et al., 2007). }\end{array}$ & $S$ \\
\hline Preference & $\begin{array}{l}\text { Some assignments are not allowed. For example, some classes' subjects } \\
\text { must not be scheduled to some periods (AVELLA et al., 2007). }\end{array}$ & $\mathrm{H}$ \\
\hline Preference & $\begin{array}{l}\text { Classes must have consecutive lectures of the same subject placed into the } \\
\text { same classroom (STEFANO; TETTAMANZI, 2001). }\end{array}$ & $\mathrm{H}$ \\
\hline Preference & $\begin{array}{l}\text { Some class' subjects may require a minimum number of consecutive dou- } \\
\text { ble lessons (SOUZA; MACULAN; OCHI, 2004; SOUSA; MORETTI; } \\
\text { PODESTÁ, 2008). }\end{array}$ & S \\
\hline Preference & $\begin{array}{l}\text { Teachers may or may not prefer specific periods to work (SCHAERF, 1999a; } \\
\text { FILHO; LORENA, 2001; JUNGINGER, 1986). }\end{array}$ & S \\
\hline Preference & $\begin{array}{l}\text { Teachers may or may not prefer specific classrooms to work (MELICIO; } \\
\text { CALDEIRA; ROSA, 2004). }\end{array}$ & S \\
\hline Preference & $\begin{array}{l}\text { Some subjects should precede or succeed other subjects (STEFANO; TET- } \\
\text { TAMANZI, 2001; NURMI; KYNGÄS, 2007). }\end{array}$ & $\mathrm{H} / \mathrm{S}$ \\
\hline Compactness & $\begin{array}{l}\text { Classes that do not have compact schedules should have as few idle periods } \\
\text { as possible (JACOBSEN; BORTFELDT; GEHRING, 2006; ZHANG et al., } \\
\text { 2010). }\end{array}$ & $\mathrm{H} / \mathrm{S}$ \\
\hline
\end{tabular}


Table 3 - continued from previous page

\begin{tabular}{|c|c|c|}
\hline Requisite & Description & Type \\
\hline Compactness & $\begin{array}{l}\text { Classes that do not have compact schedules should have their free periods at } \\
\text { the end of the days (AVELLA et al., 2007; TASSOPOULOS; BELIGIAN- } \\
\text { NIS, 2012a). }\end{array}$ & $\mathrm{H} / \mathrm{S}$ \\
\hline Compactness & $\begin{array}{l}\text { Each class' subject should have consecutive lectures when it is scheduled } \\
\text { more than once to the same day (SCHAERF, 1999a; AVELLA et al., 2007). }\end{array}$ & S \\
\hline Scattering & $\begin{array}{l}\text { Each class' subject should not have consecutive lectures within the same day } \\
\text { (BELIGIANNIS et al., 2008; KOCHETOV; KONONOVA; PASCHENKO, } \\
\text { 2008). }\end{array}$ & S \\
\hline Scattering & $\begin{array}{l}\text { Each class' subject should have their lectures uniformly distributed during } \\
\text { the week (CALDEIRA; ROSA, 1997; TASSOPOULOS; BELIGIANNIS, } \\
\text { 2012b). }\end{array}$ & S \\
\hline Compactness & $\begin{array}{l}\text { Teachers' schedules should have as few idle periods as possible (SOUZA; } \\
\text { MACULAN; OCHI, 2004; ZHANG et al., 2010). }\end{array}$ & S \\
\hline Scattering & $\begin{array}{l}\text { The total number of idle periods should be uniformly distributed among all } \\
\text { teachers, while individual teachers should have their idle periods scattered } \\
\text { during the week (BELIGIANNIS et al., 2008; TASSOPOULOS; BELI- } \\
\text { GIANNIS, 2012a). }\end{array}$ & S \\
\hline Scattering & $\begin{array}{l}\text { The teachers' weekly workload should be uniformly distributed among the } \\
\text { days (BELIGIANNIS et al., 2008). }\end{array}$ & $\mathrm{H} / \mathrm{S}$ \\
\hline Compactness & $\begin{array}{l}\text { The teachers' weekly workload should be distributed in a minimum number } \\
\text { of days (SOUZA; MACULAN; OCHI, 2004; HAAN et al., 2007). }\end{array}$ & $\mathrm{H} / \mathrm{S}$ \\
\hline Balancing & Extra working days should be balanced among teachers (SAVINIEC, 2013). & $S$ \\
\hline TravelTime & $\begin{array}{l}\text { Teachers should have enough time to travel from one building to another } \\
\text { between to consecutive lectures (SCHAERF, 1999a). }\end{array}$ & $S$ \\
\hline
\end{tabular}

\subsubsection{State-of-the-art techniques}

Since the first studies in the late 1950s, as described by Junginger (1986), the scientific literature has reported numerous papers dealing with solution techniques for high school timetabling problems. In this section, we review those that are state-of-the-art.

Up to the 2000s, the high school timetabling research had been mostly based on independent case studies, making it difficult to compare different solution methods. According to our literature review, the first high school timetabling dataset available appeared in the early 2000s. Souza (2000) introduced a small set of instances from Brazilian high schools ${ }^{1}$, which became a commonly used benchmark, being addressed later by both heuristics (SOUZA; MACULAN; OCHI, 2004; SANTOS; OCHI; SOUZA, 2005; SAVINIEC et al., 2013; DORNELES; ARAÚJO; BURIOL, 2014; SAVINIEC; CONSTANTINO, 2017) and mixed-integer programming techniques (SANTOS et al., 2012; DORNELES; ARAÚJO; BURIOL, 2014; DORNELES; ARAÚJO;

1 Available in <http://labic.ic.uff.br/Instance/> 
BURIOL, 2017). This dataset was extended with new real cases ${ }^{2}$ proposed by Saviniec and Constantino (2017). The state-of-the-art heuristic methods for this problem are fix-and-optimize heuristics (DORNELES; ARAÚJO; BURIOL, 2014) and iterated local search metaheuristics (SAVINIEC et al., 2013; SAVINIEC; CONSTANTINO, 2017).

A second high school timetabling dataset appeared in the late 2000s. Beligiannis et al. (2008) introduced a set of real-case instances from Greek high schools ${ }^{3}$ that also became the subject of several studies (BELIGIANNIS; MOSCHOPOULOS; LIKOTHANASSIS, 2009; ZHANG et al., 2010; TASSOPOULOS; BELIGIANNIS, 2012a; SKOULLIS; TASSOPOULOS; BELIGIANNIS, 2017). The state-of-the-art heuristic method reported for the Greek problem is a cat swarm optimization based algorithm (SKOULLIS; TASSOPOULOS; BELIGIANNIS, 2017).

During the PATAT conference of 2008 , the timetabling community proposed an XMLbased format to represent different high school timetabling problems and create a unified set of benchmark instances (POST et al., 2012). This format, called XHSTT (POST et al., 2014), established rules to model the most common high school timetabling requisites found around the world. In other words, the XHSTT is a format to represent a generalized high school timetabling problem (GHSTP). After its creation, new instances from different countries have been added to the XHSTT repository ${ }^{4}$, including those proposed by Souza (2000). Such instances were used in the ITC-2011. The state-of-the-art method for these instances is the heuristic solver GOAL (FONSECA et al., 2016), which won the ITC-2011. An updated version of GOAL is also described in Fonseca, Santos and Carrano (2016), which employs metaheuristic and matheuristic based algorithms.

Regarding the use of mixed-integer programming techniques, different compact formulations have been proposed for high school timetabling problems (SOUZA, 2000; SANTOS et al., 2012; DORNELES; ARAÚJO; BURIOL, 2014; SøRENSEN; DAHMS, 2014; AL-YAKOOB; SHERALI, 2015; KRISTIANSEN; SØRENSEN; STIDSEN, 2015). However, all of these formulations have shown weak linear relaxations. The strongest formulation for high school timetabling is based on a multi-commodity flow problem. The formulation was first proposed for problems of Brazilian high schools (DORNELES; ARAÚJO; BURIOL, 2017) and adapted later to manage the GHSTP (FONSECA et al., 2017). Extended formulations solved by column generation have also been proposed for high school timetabling problems. Santos et al. (2012) and Dorneles, Araújo and Buriol (2017) proposed different formulations for the Brazilian case, which showed similar results regarding the quality of their lower bounds. Another column generation was also proposed in Al-Yakoob and Sherali (2015), for problems of Kuwaiti high schools.

As mentioned before, the literature has reported many other case studies dealing with

2 Available in <http://www.gpea.uem.br/benchmark.html>

3 Available in <http://www.deapt.upatras.gr/cso_timetabling/school-timetabling.html $>$

4 Available in <https://www.utwente.nl/ctit/hstt/> 
solution techniques for high school timetabling problems. A detailed review of these methods is available in Pillay (2014). In Table 4, we sample the most recent and relevant papers by chronological order. For each paper, we identify the problem characteristics, as discussed at the beginning of Section 2.3, and the type of technique employed (see the list of abbreviations for complete names). Table 4 shows that solution techniques for high school timetabling problems have been mostly based on genetic algorithms (GA), simulated annealing (SA), tabu search (TS) and mixed-integer programming models (MIP).

Table 4 - Solution techniques for high school timetabling.

\begin{tabular}{|c|c|c|}
\hline Paper & Problem characteristics & Solution techniques \\
\hline (SOUZA, 2000) & NRA, TPS, CCS & MIP, TS, GRASP, SA \\
\hline (FILHO; LORENA, 2001) & NRA, TPS, CCS & GA \\
\hline (STEFANO; TETTAMANZI, 2001) & RA, TPS, NCCS, CT & GA \\
\hline (VALOUXIS; HOUSOS, 2003) & NRA, TPS, NCCS & $\mathrm{CP}$ \\
\hline (SOUZA; MACULAN; OCHI, 2004) & NRA, TPS, CCS & GRASP, TS \\
\hline (SANTOS; OCHI; SOUZA, 2005) & NRA, TPS, CCS & TS \\
\hline $\begin{array}{l}\text { (JACOBSEN; BORTFELDT; GEHRING, } \\
\text { 2006) }\end{array}$ & RA, TPS, NCCS & TS \\
\hline (MARTE, 2007) & RA, TPS & $\mathrm{CP}$ \\
\hline (AVELLA et al., 2007) & NRA, TPS, NCCS & MIP, SA \\
\hline (SOUSA; MORETTI; PODESTÁ, 2008) & NRA, TPS, CCS & MIP, TS \\
\hline (RAGHAVJEE; PILLAY, 2008) & RA, TPS & GA \\
\hline (BELIGIANNIS et al., 2008) & NRA, TPS, NCCS, STC, CT & GA \\
\hline (CERDEIRA-PENA et al., 2008) & NRA, TPS, STC & GA \\
\hline (BELLO; RANGEL; BOERES, 2008) & NRA, TPS, CCS & $\mathrm{TS}$ \\
\hline (WILKE; OSTLER, 2008) & RA & TS, SA, GA, B\&B \\
\hline (LARA; FLORES; CALDERÓN, 2008) & NRA, TPS, NCCS & $\mathrm{BA}$ \\
\hline $\begin{array}{ll}\text { (KOCHETOV; } & \text { KONONOVA; } \\
\text { PASCHENKO, 2008) } & \end{array}$ & NRA, TPS & VNS, TS \\
\hline $\begin{array}{l}\text { (BIRBAS; DASKALAKI; HOUSOS, } \\
\text { 2009) }\end{array}$ & NRA, TPS, NCCS, STC, CT & MIP \\
\hline (RAGHAVJEE; PILLAY, 2009) & NRA, TPS, NCCS, STC, CT & GA \\
\hline (PILLAY, 2010) & RA, TPS & $\mathrm{HH}, \mathrm{GA}$ \\
\hline (RAGHAVJEE; PILLAY, 2010a) & NRA, TPS, STC & GA \\
\hline (ZHANG et al., 2010) & NRA, NTPS or TPS, NCCS, STC, CT & SA \\
\hline (RAGHAVJEE; PILLAY, 2010b) & NRA, TPS, NCCS, STC & GA \\
\hline (SANTOS et al., 2012) & NRA, TPS, CCS & CCG \\
\hline (DORNELES; ARAÚJO; BURIOL, 2012) & NRA, TPS, CCS & MIP \\
\hline (BRITO et al., 2012) & GHSTP & SA, VNS \\
\hline (SAVINIEC et al., 2013) & NRA, TPS, CCS & ILS \\
\hline (DORNELES; ARAÚJO; BURIOL, 2014) & NRA, TPS, CCS & MIP, F\&O \\
\hline (FONSECA; SANTOS, 2014) & GHSTP & VNS \\
\hline (SØRENSEN; STIDSEN, 2014) & GHSTP & $\mathrm{F} \& \mathrm{O}$ \\
\hline (SøRENSEN; DAHMS, 2014) & RA & TSD-MIP \\
\hline
\end{tabular}


Table4 - continued from previous page

\begin{tabular}{l|l|l}
\hline Paper & Problem characteristics & Solution techniques \\
\hline (KRISTIANSEN; SøRENSEN; STIDSEN, & GHSTP & MIP \\
2015) & & \\
(AHMED; ÖZCAN; KHEIRI, 2015) & GHSTP & HH \\
(AL-YAKOOB; SHERALI, 2015) & NRA, NTPS, NCCS & MIP, CG \\
(FONSECA et al., 2016) & GHSTP & SA, ILS \\
(FONSECA; SANTOS; CARRANO, 2016) & GHSTP & SA, LAS \\
(FONSECA; SANTOS; CARRANO, 2016) & GHSTP & VNS, F\&O \\
(DORNELES; ARAÚJO; BURIOL, 2017) & NRA, TPS, CCS & MIP, CG \\
(DEMIROVIć; MUSLIU, 2017) & GHSTP & LS-MaxSAT \\
(FONSECA et al., 2017) & GHSTP & MIP \\
(SKOULLIS; TASSOPOULOS; BELI- & NRA, TPS, NCCS, STC, CT & CSO \\
GIANNIS, 2017) & & \\
(SAVINIEC; CONSTANTINO, 2017) & NRA, TPS, CCS & ILS, VNS \\
\hline
\end{tabular}

\subsection{The problem under study}

We focus on real timetabling problems motivated by rules of Brazilian high schools. As mentioned before, the first Brazilian HSTP reported in the scientific literature was introduced by Souza (2000). The author proposed a compact MIP formulation and instances that represent the main characteristics of high school timetabling problems arising in Brazilian schools. His work showed that, at that time, even small size instances were a challenge for MIP solvers. Since then, the introduced instances became a benchmark for tests, and several other studies addressed the problem.

Souza, Maculan and Ochi (2004) proposed the first metaheuristic approach to deal with the problem introduced by Souza (2000). The proposed method is a hybrid algorithm which employs a GRASP procedure to construct an initial solution that is improved by a tabu search. Following, Santos, Ochi and Souza (2005) proposed a tabu search with two memory based diversification strategies that showed to outperform the hybrid approach of Souza, Maculan and Ochi (2004).

After the attempt of Souza (2000) to solve the Brazilian HSTP via a compact MIP formulation, Santos et al. (2012) proposed an extended formulation that is solved by column generation. Additional cuts generated by the Fenchel cutting planes technique (BOYD, 1994a; BOYD, 1994b) are added to improve their formulation. The work of Santos et al. (2012) provided, for the first time, strong lower bounds and some optimal solutions to the problem when compared to previous solutions found by the tabu search of Santos, Ochi and Souza (2005). 
Later, Saviniec et al. (2013) showed that the lower bounds of Santos et al. (2012) were indeed the optimal values for all instances by comparing with solutions found by iterated local search based algorithms. Afterward, Dorneles, Araújo and Buriol (2014) found similar results via fix-and-optimize heuristics.

The latest studies addressing the problem are the works of Dorneles, Araújo and Buriol (2017) and Saviniec and Constantino (2017). Dorneles, Araújo and Buriol (2017) proposed a compact and an extended formulation based on a multi-commodity flow problem. The extended formulation is solved by column generation, and the generated lower bounds were reported to be equivalent to the lower bounds found by Santos et al. (2012). Saviniec and Constantino (2017) proposed new instances and algorithms based on iterated local search and variable neighborhood search that showed better results than the previous approaches when tested on the instances proposed by Souza (2000).

The problem introduced by Souza (2000) was also addressed in the ITC-2011 with a slight modification. The ITC-2011 addressed several high school timetabling variants from other countries, which differ from the Brazilian case in timetabling rules.

One of the most important requisites in Brazilian HSTP is to obtain compact schedules for teachers (SOUZA, 2000). Saviniec (2013) considered a case with balancing on teachers' extra worked days. Other requisites are also present in Brazilian HSTP, such as availability of teachers, preference for double lessons, and avoiding idle periods for teachers.

In this thesis, we consider the three HSTP variants mentioned above. We will refer to these problems as HSTP-A - the problem introduced by Saviniec (2013), HSTP-B - the problem introduced by Souza (2000), and HSTP-C - the version of the problem of Souza (2000) included in the ITC-2011. In Section 2.4.1, we present the definition of these problems. The problems themselves and their objective functions are modeled in Chapter 3.

\subsubsection{Definition}

The high school timetabling problems considered in this thesis are structured as shown in Figure 4. The school has a set of classes $C$ and a set of teachers $T$. Classes are disjoint groups of students enrolled in the same list of subjects (e.g., mathematics, physics, etc.) and with no free periods during the week. Each class' subject has a number of weekly meetings (or lessons) that are taught by a preassigned teacher. The set of all classes' subjects is called "Required meetings" $(R)$ and each class' subject is called a "requirement". Teachers may also be unavailable during some days and periods. Given these input data, the goal is to obtain weekly timetable specifying the schedule for all required meetings in a particular shift (morning, afternoon, or evening). A timetable is constructed for each independent shift, and it usually spreads over the set $D$ of weekdays (Monday to Friday) with a set $H$ of five periods per day. Throughout this thesis, we also refer to periods as a set of timeslots $(d, h) \in D \times H$. 


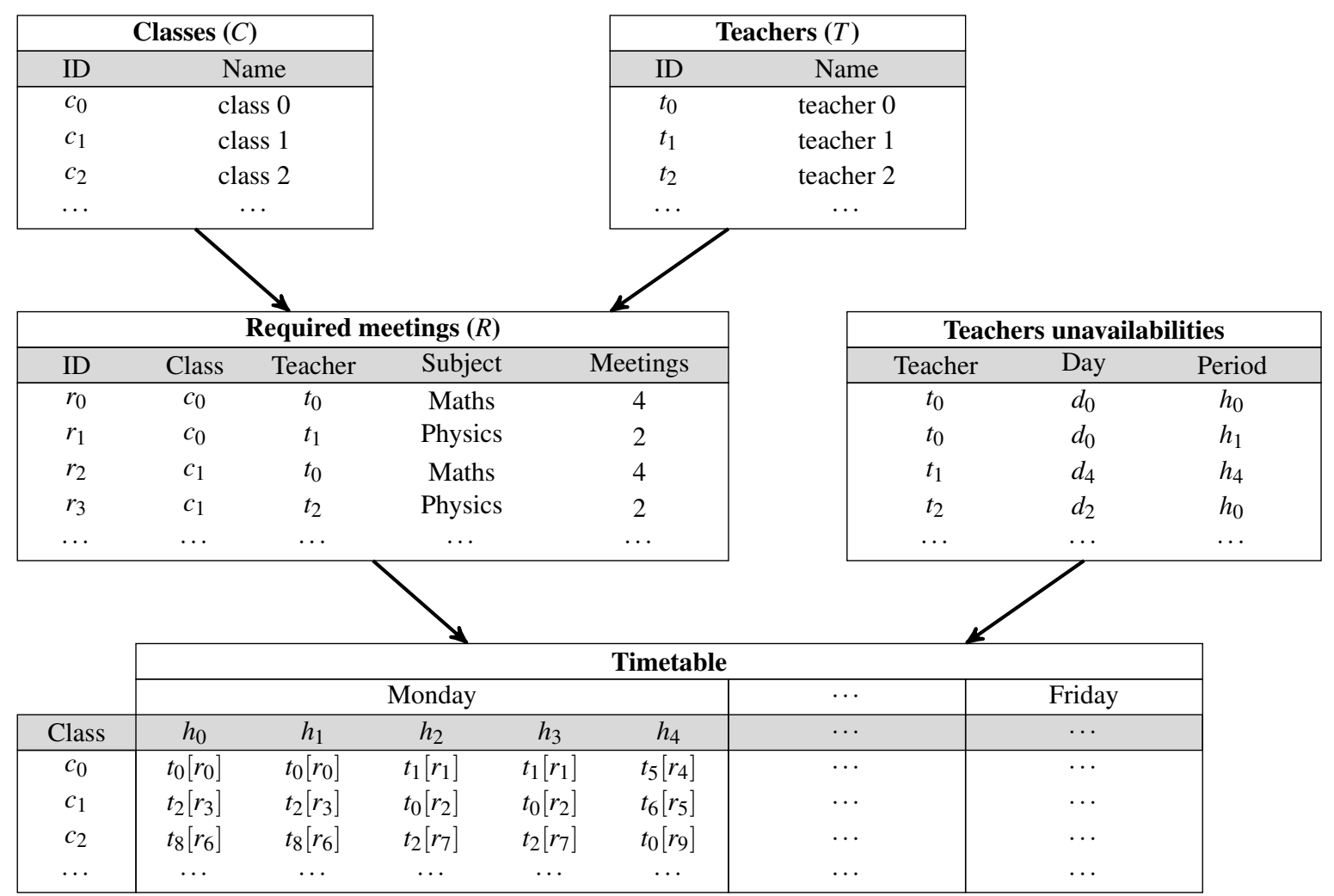

Figure 4 - High school timetabling structure.

Given these definitions, the timetabling process must fulfill the following requisites:

1. Meeting of weekly required lessons: all required lessons must be assigned.

2. No clashes in classes' schedules: each class $c \in C$ must attend exactly one lesson per period.

3. No clashes in teachers' schedules: each teacher $t \in T$ must teach at most one lesson per period.

4. No assignment of teachers in their unavailable periods: teachers must not be assigned to periods in which they are unavailable.

5. No daily workload violation for requirements: for each requirement $r \in R$, there is a limit $\widetilde{\delta}_{r}$ on the amount of meetings per day. Therefore, daily workloads greater than $\widetilde{\delta}_{r}$ should be avoided or are forbidden.

6. No holes in requirements' schedules: holes in requirements' schedules should be avoided or are forbidden. A hole is a period splitting the daily schedule of a requirement into nonconsecutive meetings.

7. Meeting of double lessons for requirements: for each requirement $r \in R$, there is a specified minimum number of consecutive double lessons $\widetilde{\pi}_{r}$ that should be met. 
8. No idle periods in teachers' schedules: teachers should not have idle periods in their daily schedules.

9. Compact schedules for teachers: teachers should be scheduled to come to school the minimum possible number of days.

10. Balancing on teachers' extra working days: the extra working days should be balanced among teachers. The number of extra working days for a teacher $t$ is the difference between the number of worked days and an estimated minimum $\widetilde{d}_{t}$, defined later in Section 3.1.

Depending on the considered variant HSTP-A, HSTP-B or HSTP-C, some of these requisites may be hard or soft, and we model them in Chapter 3. 



\section{MIXED-INTEGER PROGRAMMING MODELS}

In this chapter, we propose five mixed-integer programming formulations for the Brazilian HSTP variants described in Section 2.4. For two of these formulations (Section 3.1), the variables can be explicitly generated and solved in a black-box MIP solver. For the three others (Section $3.2)$, there is a huge number of variables, and we resort to the column generation technique to solve their linear relaxation, which provide tight lower bounds on the optimal integer solution of problem instances.

\subsection{Compact formulations}

This section presents the two formulations for which the variables can be explicitly generated. Let us consider the following parameters, that represent a high school timetabling instance.

- $C$ : a set of classes.

- $T$ : a set of teachers.

- $D$ : a set of weekdays.

- $H$ : a set of teaching periods per day.

- $H_{t d}$ : a set of periods in day $d \in D$ for which teacher $t \in T$ is available.

- $R$ : the set of required meetings (or lessons), as defined in Section 2.4.1.

- $R_{c}$ : the set of requirements that belong to class $c \in C$.

- $R_{t}$ : the set of requirements that belong to teacher $t \in T$.

- $\widetilde{\theta}_{r}$ : the number of weekly lessons of requirement $r \in R$. 
- $\widetilde{\delta}_{r}$ : a daily limit for lessons of requirement $r \in R$.

- $\tilde{\pi}_{r}$ : the desired number of consecutive double lessons of requirement $r \in R$.

- $\widetilde{d}_{t}=\left\lceil\frac{\sum_{r \in R_{t}} \widetilde{\theta}_{r}}{|H|}\right\rceil:$ the estimated minimum number of days in which teacher $t \in T$ can be assigned and still meet his/her teaching requirements.

\subsubsection{Formulation CF1}

In this compact formulation, a timetable output can be represented by a set of binary variables $x_{r d h}$, for all $(r, d, h) \in R \times D \times H$. Table 5 describes the notation used in formulation $\mathrm{CF} 1$ and the HSTP requisites can be formulated as:

Table 5 - Notation used in formulation CF1.

\begin{tabular}{ll}
\hline Notation & Definition \\
\hline $\begin{array}{l}\text { Decision variables } \\
x_{r d h} \in\{0,1\}\end{array}$ & indicates whether requirement $r \in R$ is assigned to period $h \in H$ of day $d \in D$. \\
Auxiliary variables & \\
$\widehat{\delta}_{r d} \in \mathbb{N}$ & the number of lessons that exceeds the limit $\widetilde{\delta}_{r}$ in day $d$ for requirement $r$. \\
$\hat{j}_{r d h} \in\{0,1\}$ & indicates whether the schedule of requirement $r$ has a hole at period $h$ of day $d$. \\
$\widehat{\phi}_{r d h} \in\{0,1\}$ & indicates whether requirement $r$ has a double lesson at periods $h-1$ and $h$ of day $d$. \\
$\widehat{\pi}_{r} \in \mathbb{N}$ & the number of unmet weekly double lessons of requirement $r$. \\
$\widehat{d}_{t d} \in\{0,1\}$ & indicates whether teacher $t$ works in day $d$. \\
$\widehat{i}_{t d k} \in\{0,1\}$ & indicates whether teacher $t$ is idle at period $k$ of day $d$. \\
$\widehat{\beta} \in \mathbb{N}$ & the greatest value among all teachers' extra working days. \\
\hline
\end{tabular}

- Meeting of weekly required lessons:

$$
\sum_{d \in D} \sum_{h \in H} x_{r d h}=\widetilde{\theta}_{r} \quad \forall r \in R
$$

- No clashes in classes' schedules:

$$
\sum_{r \in R_{c}} x_{r d h}=1 \quad \forall c \in C ; d \in D ; h \in H
$$

\section{- No clashes in teachers' schedules:}

$$
\sum_{r \in R_{t}} x_{r d h} \leq 1 \quad \forall t \in T ; d \in D ; h \in H_{t d}
$$


- No assignment of teachers in their unavailable periods:

$$
\sum_{r \in R_{t}} x_{r d h}=0 \quad \forall t \in T ; d \in D ; h \in H \backslash H_{t d}
$$

- No daily workload violation for requirements: this requisite may appear in both forms.

- Hard requisite: for each requirement $r \in R$, daily workloads greater than $\widetilde{\delta}_{r}$ are forbidden (SOUZA, 2000; SOUSA; MORETTI; PODESTÁ, 2008).

$$
\sum_{h \in H} x_{r d h} \leq \widetilde{\delta}_{r} \quad \forall r \in R ; d \in D
$$

- Soft requisite: for each requirement $r \in R$, daily workloads greater than $\widetilde{\delta}_{r}$ should be avoided (SAVINIEC, 2013). Violations of this requisite can be measured by the auxiliary variables $\widehat{\delta}_{r d}$.

$$
\begin{aligned}
\widehat{\delta}_{r d} \geq \sum_{h \in H} x_{r d h}-\widetilde{\boldsymbol{\delta}}_{r} & \forall r \in R ; d \in D \\
\widehat{\delta}_{r d} \geq 0 & \forall r \in R ; d \in D
\end{aligned}
$$

- No holes in requirements' schedules: this requisite may appear in both forms.

- Hard requisite: holes in requirements' schedules are forbidden (DORNELES; ARAÚJO; BURIOL, 2017).

$$
\begin{aligned}
x_{r d i}-x_{r d h}+x_{r d j}-1 \leq 0 \quad & \forall r \in R ; d \in D ; h=1, \cdots,|H|-2 ; \\
& i=0, \cdots, h-1 ; j=h+1, \cdots,|H|-1
\end{aligned}
$$

- Soft requisite: holes in requirements' schedules should be avoided (SOUSA; MORETTI; PODESTÁ, 2008; SAVINIEC, 2013). For each requirement $r \in R$, a hole in period $h \in\{1, \ldots,|H|-2\}$ of day $d$ is flagged by the auxiliary variables $\widehat{j}_{r d h}$.

$$
\begin{aligned}
& \widehat{j}_{r d h} \geq x_{r d i}-x_{r d h}+x_{r d j}-1 \quad \forall r \in R ; d \in D ; h=1, \cdots,|H|-2 ; \\
& i=0, \cdots, h-1 ; j=h+1, \cdots,|H|-1 \\
& \widehat{j}_{r d h} \geq 0 \quad \forall r \in R ; d \in D ; h=1, \cdots,|H|-2
\end{aligned}
$$


- Meeting of double lessons for requirements: for each requirement $r \in R$, the auxiliary variables $\widehat{\phi}_{r d h}$ are set to "1" if a double lesson ends in periods $h \in\{1, \cdots,|H|-1\}$ within each day, while variables $\widehat{\pi}_{r}$ quantify the number of unmet weekly double lessons.

$$
\begin{aligned}
\widehat{\phi}_{r d h} \leq x_{r d h} & \forall r \in R ; d \in D ; h=1, \cdots,|H|-1 \\
\widehat{\phi}_{r d h} \leq x_{r, d, h-1} & \forall r \in R ; d \in D ; h=1, \cdots,|H|-1 \\
\widehat{\phi}_{r d h} \leq 1-\widehat{\phi}_{r, d, h-1} & \forall r \in R ; d \in D ; h=2, \cdots,|H|-1 \\
\widehat{\pi}_{r} \geq \widetilde{\pi}_{r}-\sum_{d \in D} \sum_{h=1}^{|H|-1} \widehat{\phi}_{r d h} & \forall r \in R \\
\widehat{\phi}_{r d h} \geq 0 & \forall r \in R ; d \in D ; h=1, \cdots,|H|-1 \\
\widehat{\pi}_{r} \geq 0 & \forall r \in R
\end{aligned}
$$

- No idle periods in teachers' schedules: For each teacher $t \in T$ and day $d \in D$, idle periods $k$ are flagged by the auxiliary variables $\widehat{i}_{t d k}$. There are two cases for this requisite.

- A teacher may be idle at period $k \in H$ even if he/she is unavailable in such period (SOUZA, 2000).

$$
\begin{aligned}
\hat{i}_{t d k} \geq \sum_{r \in R_{t}}\left(x_{r d i}-x_{r d k}+x_{r d j}\right)-1 & \forall t \in T ; d \in D ; k=1, \cdots,|H|-2 ; \\
& i=0, \cdots, k-1 ; j=k+1, \cdots,|H|-1 \\
\widehat{i}_{t d k} \geq 0 & \forall t \in T ; d \in D ; k=1, \cdots,|H|-2
\end{aligned}
$$

- A teacher may be idle at period $k \in H$ only if he/she is available at such period (SAVINIEC, 2013).

$$
\begin{aligned}
& \widehat{i}_{t d k} \geq \sum_{r \in R_{t}}\left(x_{r, d, h_{i}}-x_{r, d, h_{k}}+x_{r, d, h_{j}}\right)-1 \quad \forall t \in T ; d \in D ; k=1, \cdots,\left|H_{t d}\right|-2 ; \\
& i=0, \cdots, k-1 ; j=k+1, \cdots,\left|H_{t d}\right|-1 ; \\
& h_{i}, h_{k}, h_{j} \in H_{t d} \\
& \widehat{i}_{t d k} \geq 0 \quad \forall t \in T ; d \in D ; k=1, \cdots,\left|H_{t d}\right|-2
\end{aligned}
$$

Inequalities (3.19) are similar to inequalities (3.17), except that we omit variable $\widehat{i}$ for periods in which the teacher is unavailable. For example, consider that the set of periods is $H=\{0,1,2,3,4\}$ and suppose that teacher $t \in T$ is not available to teach at periods 1 and 2 within day $d \in D$, then $H_{t d}=\{0,3,4\}$. Now, only variable $\widehat{i_{t, d}, 3}$ and related constraints are taken into account. 
- Compact schedules for teachers: For each teacher $t \in T$, the auxiliary variables $\widehat{d}_{t d}$ flag if he/she works in day $d \in D$. The sum of these variables is minimized later in the objective function to obtain compact schedules.

$$
\begin{aligned}
& \widehat{d_{t d}} \geq \sum_{r \in R_{t}} x_{r d h} \quad \forall t \in T ; d \in D ; h \in H_{t d} \\
& \widehat{d_{t d}} \geq 0 \quad \forall t \in T ; d \in D
\end{aligned}
$$

- Balancing on teachers' extra working days: to create schedules in which the extra working days are balanced among teachers, we minimize the auxiliary variable $\widehat{\beta}$ which captures the greatest value among teachers' extra worked days.

$$
\begin{gathered}
\widehat{\beta} \geq \sum_{d \in D} \widehat{d}_{t d}-\widetilde{d}_{t} \quad \forall t \in T \\
\widehat{\beta} \geq 0
\end{gathered}
$$

\subsubsection{Problem HSTP-A}

This is the problem proposed by Saviniec (2013). Apart from constraints to obtain compact schedules for teachers, the problem also includes constraints to obtain timetables in which the extra worked days are balanced among the teachers. The complete mixed-integer program can be written as:

$$
\begin{aligned}
\text { Minimize } & \alpha_{5} \sum_{r \in R} \sum_{d \in D} \widehat{\delta}_{r d}+\alpha_{6} \sum_{r \in R} \sum_{d \in D} \sum_{h=1}^{|H|-2} \widehat{j}_{r d h}+\alpha_{7} \sum_{r \in R} \widehat{\pi}_{r} \\
& +\alpha_{8} \sum_{t \in T} \sum_{d \in D} \sum_{k=1}^{\left|H_{t d}\right|-2} \widehat{i}_{t d k}+\alpha_{9} \sum_{t \in T} \sum_{d \in D} \widehat{d}_{t d}+\alpha_{10} \cdot \widehat{\beta}
\end{aligned}
$$

Subject to: 
(3.1) Meeting of weekly required lessons

(3.2) No clashes in classes' schedules

(3.3) No clashes in teachers' schedules

(3.4) No assignment of teachers in their unavailable periods

(3.6) - (3.7) No daily workload violation for requirements

(3.9) - (3.10) No holes in requirements' schedules

(3.11) - (3.16) Meeting of double lessons for requirements

$(3.19)-(3.20) \quad$ No idle periods in teachers' schedules

(3.21) - (3.22) Compact schedules for teachers

(3.23) - (3.24) Balancing on teachers' extra working days

$x_{r d h} \in\{0,1\} \quad \forall r \in R ; d \in D ; h \in H$

The goal of this problem is to satisfy requisites 1 to 4, defined in Section 2.4.1, while minimizes the violations of requisites 5 to 10. In the objective function (3.25), the constant $\alpha_{i}$ is a penalty that expresses the importance of satisfying the $i^{\text {th }}$ requisite, for $i=5, \cdots, 10$.

The MIP program (3.25) - (3.26) is also augmented with the additional cuts (3.27) which specify that each teacher $t$ cannot work less than the minimum number of working days $\widetilde{d}_{t}$ (SOUZA, 2000).

$$
\sum_{d \in D} \widehat{d}_{t d} \geq \widetilde{d}_{t} \quad \forall t \in T
$$

\subsubsection{Problem HSTP-B}

This is the problem proposed by Souza (2000). The author does not consider the "balancing on teachers" extra working days" and the requisite "no daily workload violation for requirements" is a hard requisite. The complete mixed-integer program can be written as:

Minimize $\quad \alpha_{7} \sum_{r \in R} \widehat{\pi}_{r}+\alpha_{8} \sum_{t \in T} \sum_{d \in D} \sum_{k=1}^{|H|-2} \widehat{i}_{t d k}+\alpha_{9} \sum_{t \in T} \sum_{d \in D} \widehat{d}_{t d}$

Subject to: 
(3.1) Meeting of weekly required lessons

(3.2) No clashes in classes' schedules

(3.3) No clashes in teachers' schedules

(3.4) No assignment of teachers in their unavailable periods

(3.5) No daily workload violation for requirements

(3.11) - (3.16) Meeting of double lessons for requirements

(3.17) - (3.18) No idle periods in teachers' schedules

(3.21) - (3.22) Compact schedules for teachers

$x_{r d h} \in\{0,1\} \quad \forall r \in R ; d \in D ; h \in H$

The goal of this problem is to satisfy requisites 1 to 5 , while minimizes the violations of requisites 7 to 9. The MIP program (3.28) - (3.29) is also augmented with additional cuts to specify that teachers do not work less than a minimum number of working days (SOUZA, 2000). The cuts are given by inequalities (3.30):

$$
\sum_{d \in D} \widehat{d}_{t d} \geq \max \left\{\widetilde{d}_{t}, \max _{r \in R_{t}}\left\{\left\lceil\frac{\widetilde{\theta}_{r}}{\widetilde{\delta}_{r}}\right\rceil\right\}\right\} \quad \forall t \in T
$$

\subsubsection{Problem HSTP-C}

This is the variant of the problem of Souza (2000) included in the ITC-2011. Problem HSTP-C has an additional hard requisite to ensure that there are "no holes in requirements' schedules", so that the lessons are always consecutive within each day. The complete mixedinteger program can be written as:

Minimize $\quad \alpha_{7} \sum_{r \in R} \widehat{\pi}_{r}+\alpha_{8} \sum_{t \in T} \sum_{d \in D} \sum_{k=1}^{|H|-2} \widehat{i}_{t d k}+\alpha_{9} \sum_{t \in T} \sum_{d \in D} \widehat{d}_{t d}$ 
(3.1) Meeting of weekly required lessons

(3.2) No clashes in classes' schedules

(3.3) No clashes in teachers' schedules

(3.4) No assignment of teachers in their unavailable periods

(3.5) No daily workload violation for requirements

(3.8) No holes in requirements' schedules

(3.11) - (3.16) Meeting of double lessons for requirements

$(3.17)-(3.18) \quad$ No idle periods in teachers' schedules

(3.21) - (3.22) Compact schedules for teachers

$x_{r d h} \in\{0,1\} \quad \forall r \in R ; d \in D ; h \in H$

The goal of this problem is to satisfy requisites 1 to 6 , while minimizes the violations of requisites 7 to 9. The MIP program (3.31) - (3.32) is also augmented by cuts (3.30).

\subsubsection{Formulation CF2}

Table 6 - An illustration of all possible assignment layouts in a day with four teaching periods.

\begin{tabular}{rrl}
\hline Integer ID & Layout & Comments \\
\hline 0 & 0000 & a day off. \\
2 & 0001 & an assignment at period $h_{3}$. \\
3 & 0010 & \\
4 & 0011 & \\
5 & 0100 & an assignment at period $h_{1}$. \\
6 & 0110 & \\
7 & 0111 & for a requirement, this layout exceeds a daily limit of two lessons. \\
8 & 1000 & an assignment at period $h_{0}$. \\
9 & 1001 & for a teacher, he/she is idle at periods $h_{1}$ and $h_{2}$. \\
10 & 1010 & for a requirement, there is a hole at period $h_{1}$ (non-consecutive lessons). \\
11 & 1011 & \\
12 & 1100 & a double lesson at periods $h_{0}$ and $h_{1}$. \\
13 & 1101 & \\
14 & 1110 & \\
15 & 1111 & a full-day assignment layout.
\end{tabular}

In this section, we propose an alternative formulation that follows the idea of layouts, in which daily schedules of teachers and requirements are represented by assignment layouts. A daily assignment layout is a binary vector of size $|H|$ in which the $h^{\text {th }}$ position represents the $h^{\text {th }}$ period of the day. A bit value " 1 " represents an assignment in the $h^{\text {th }}$ period, while a bit value "0" represents no assignment. An illustration is shown in Table 6, for a day with four teaching periods. In this formulation, all possible number of layouts for a requirement or teacher is given by $|D| \cdot 2^{|H|}$. Since $|H|$ is usually a small constant for most high school timetabling problems, in our case $|H|=5$, we can explicitly enumerate all variables. 
Table 7 - Notation used in formulation CF2.

\begin{tabular}{ll}
\hline Notation & Definition \\
\hline Sets & the set of assignment layouts that a requirement $r$ (or teacher $t$ ) can follow in a day. \\
$L$ &
\end{tabular}

\section{Parameters}

$\widetilde{a}_{l} \in \mathbb{N}$

$\widetilde{\omega}_{l} \in \mathbb{N}$

$\widetilde{h}_{l} \in \mathbb{N}$

$\widetilde{e}_{r l} \in \mathbb{N}$

$\tilde{i}_{\text {t } d l} \in \mathbb{N}$

$\widetilde{\mu}_{l h} \in\{0,1\}$

$\widetilde{\phi}_{l} \in\{0,1\}$

the number of assignments in layout $l \in L$.

the number of consecutive double lessons in layout $l$.

the number of holes in layout $l$ for requirements.

the number of lessons that exceed the daily limit $\widetilde{\delta}_{r}$ in layout $l$ for a requirement $r$.

the number of idle periods in layout $l$ for teacher $t$ on day $d$.

indicates whether layout $l$ has an assignment at period $h$.

indicates whether the number of assignments in layout $l$ is greater than zero.

\section{Decision variables}

$x_{r d l} \in\{0,1\}$

$y_{t d l} \in\{0,1\}$

Auxiliary variables

$\widehat{\pi}_{r} \in \mathbb{N}$

$\widehat{\beta} \in \mathbb{N}$ indicates whether requirement $r$ follows layout $l$ on day $d$.

indicates whether teacher $t$ follows layout $l$ on day $d$.

Given the above definitions and the auxiliary notation presented in Table 7, a timetable output can be represented by a set of binary variables $x_{r d l}$ and $y_{t d l}$. These variables indicate the daily assignment layouts $l \in\left\{0,1, \cdots, 2^{|H|}-1\right\}$ that each requirement $r \in R$ and teacher $t \in T$ must follow in each day $d \in D$, respectively. Therefore, the HSTP requisites can be reformulated as:

the number of unmet weekly double lessons of requirement $r$. the greatest value among all teachers' extra working days.

- Meeting of weekly required lessons:

$$
\sum_{d \in D} \sum_{l \in L} \widetilde{a}_{l} x_{r d l}=\widetilde{\theta}_{r} \quad \forall r \in R
$$

- No clashes in classes' schedules:

$$
\sum_{r \in R_{c}} \sum_{l \in L} \widetilde{\mu}_{l h} x_{r d l}=1 \quad \forall c \in C ; d \in D ; h \in H
$$

- Only one layout per day: requirements and teachers must follow only one layout per day.

$$
\begin{array}{ll}
\sum_{l \in L} x_{r d l}=1 & \forall r \in R ; d \in D \\
\sum_{l \in L} y_{t d l}=1 & \forall t \in T ; d \in D
\end{array}
$$

Constraints (3.36) also ensure that each teacher $t \in T$ teaches at most one lesson per period. 
- No assignment of teachers in their unavailable periods: we ensure this requisite by preprocessing variables of layouts which contain assignments in periods that teachers are unavailable to teach.

$$
\begin{array}{ll}
y_{t d l}=0 & \forall t \in T ; d \in D ; l \in L ; h \in H \backslash H_{t d} ; \widetilde{\mu}_{l h}>0 \\
x_{r d l}=0 & \forall t \in T ; r \in R_{t} ; d \in D ; l \in L ; h \in H \backslash H_{t d} ; \widetilde{\mu}_{l h}>0
\end{array}
$$

- No daily workload violation for requirements:

- Hard requisite: we ensure this requisite by setting to zero the variables of layouts in which the number of assignments $\widetilde{a}_{l}$ exceeds the daily limit $\widetilde{\delta}_{r}$.

$$
x_{r d l}=0 \quad \forall r \in R ; d \in D ; l \in L ; \widetilde{a}_{l}>\widetilde{\delta}_{r}
$$

- Soft requisite: the number of violations of this requisite is computed by $\sum_{r \in R} \sum_{d \in D} \sum_{l \in L} \widetilde{e}_{r l} x_{r d l}$.

- No holes in requirements' schedules:

- Hard requisite: we ensure this requisite by setting to zero the variables of layouts that contain holes.

$$
x_{r d l}=0 \quad \forall r \in R ; d \in D ; l \in L ; \widetilde{h}_{l}>0
$$

- Soft requisite: the number of violations of this requisite is computed by $\sum_{r \in R} \sum_{d \in D} \sum_{l \in L} \widetilde{h}_{l} x_{r d l}$.

- Meeting of double lessons for requirements:

$$
\begin{aligned}
\widehat{\pi}_{r} \geq \widetilde{\pi}_{r}-\sum_{d \in D} \sum_{l \in L} \widetilde{\omega}_{l} x_{r d l} & \forall r \in R \\
\widehat{\pi}_{r} \geq 0 & \forall r \in R
\end{aligned}
$$

- No idle periods in teachers' schedules: the number of violations of this requisite is computed by $\sum_{t \in T} \sum_{d \in D} \sum_{l \in L} \widetilde{i}_{t d l} y_{t d l}$.

- Compact schedules for teachers: the number of violations of this requisite is computed by $\sum_{t \in T} \sum_{d \in D} \sum_{l \in L} \widetilde{\phi}_{l} y_{t d l}$. 
- Balancing on teachers' extra working days:

$$
\begin{gathered}
\widehat{\beta} \geq \sum_{d \in D} \sum_{l \in L} \widetilde{\phi}_{l} y_{t d l}-\widetilde{d}_{t} \quad \forall t \in T \\
\widehat{\beta} \geq 0
\end{gathered}
$$

- Linking constraints: the daily schedules of teachers must match their requirements' schedules.

$$
\sum_{l \in L} \widetilde{\mu}_{l h y_{t d l}}=\sum_{r \in R_{t}} \sum_{l \in L} \widetilde{\mu}_{l h} x_{r d l} \quad \forall t \in T ; d \in D ; h \in H
$$

\subsubsection{Problem HSTP-A}

The reformulated model for problem HSTP-A can be written as:

$$
\begin{aligned}
\text { Minimize } & \sum_{r \in R} \sum_{d \in D} \sum_{l \in L}\left(\alpha_{5} \widetilde{e}_{r l}+\alpha_{6} \widetilde{h}_{l}\right) x_{r d l}+\alpha_{7} \sum_{r \in R} \widehat{\pi}_{r} \\
& +\sum_{t \in T} \sum_{d \in D} \sum_{l \in L}\left(\alpha_{8} \widetilde{i}_{t d l}+\alpha_{9} \widetilde{\phi}_{l}\right) y_{t d l}+\alpha_{10} \widehat{\beta}
\end{aligned}
$$

Subject to:

(3.33) Meeting of weekly required lessons

(3.34) No clashes in classes' schedules

(3.35) Only one layout per day for requirements

(3.36) Only one layout per day for teachers

(3.37) - (3.38) No assignment of teachers in their unavailable periods

(3.41) - (3.42) Meeting of double lessons for requirements

(3.43) - (3.44) Balancing on teachers' extra working days

(3.45) Linking constraints

$x_{r d l} \in\{0,1\} \quad \forall r \in R ; d \in D ; l \in L$

$y_{t d l} \in\{0,1\} \quad \forall t \in T ; d \in D ; l \in L$

The cuts (3.27) become:

$$
\sum_{d \in D} \sum_{l \in L} \widetilde{\phi}_{l} y_{t d l} \geq{\widetilde{d_{t}}} \quad \forall t \in T
$$




\subsubsection{Problem HSTP-B}

The reformulated model for problem HSTP-B can be written as:

Minimize $\quad \alpha_{7} \sum_{r \in R} \widehat{\pi}_{r}+\sum_{t \in T} \sum_{d \in D} \sum_{l \in L}\left(\alpha_{8} \widetilde{i}_{t d l}+\alpha_{9} \widetilde{\phi}_{l}\right) y_{t d l}$

Subject to:

(3.33) Meeting of weekly required lessons

(3.34) No clashes in classes' schedules

(3.35) Only one layout per day for requirements

(3.36) Only one layout per day for teachers

(3.37) - (3.38) No assignment of teachers in their unavailable periods

(3.39) No daily workload violation for requirements

(3.41) - (3.42) Meeting of double lessons for requirements

(3.45) Linking constraints

$x_{r d l} \in\{0,1\} \quad \forall r \in R ; d \in D ; l \in L$

$y_{\text {tdl }} \in\{0,1\} \quad \forall t \in T ; d \in D ; l \in L$

The cuts (3.30) become:

$$
\sum_{d \in D} \sum_{l \in L} \widetilde{\phi}_{l} y_{t d l} \geq \max \left\{\widetilde{d}_{t}, \max _{r \in R_{t}}\left\{\left\lceil\frac{\widetilde{\theta}_{r}}{\widetilde{\delta}_{r}}\right\rceil\right\}\right\} \quad \forall t \in T
$$

\subsubsection{Problem HSTP-C}

The reformulated model for problem HSTP-C can be written as:

Minimize $\quad \alpha_{7} \sum_{r \in R} \widehat{\pi}_{r}+\sum_{t \in T} \sum_{d \in D} \sum_{l \in L}\left(\alpha_{8} \widetilde{i}_{t d l}+\alpha_{9} \widetilde{\phi}_{l}\right) y_{t d l}$ 
(3.33) Meeting of weekly required lessons

(3.34) No clashes in classes' schedules

(3.35) Only one layout per day for requirements

(3.36) Only one layout per day for teachers

$(3.37)-(3.38) \quad$ No assignment of teachers in their unavailable periods

(3.39) No daily workload violation for requirements

(3.40) No holes in requirements' schedules

(3.41) - (3.42) Meeting of double lessons for requirements

(3.45) Linking constraints

$x_{r d l} \in\{0,1\} \quad \forall r \in R ; d \in D ; l \in L$

$y_{t d l} \in\{0,1\} \quad \forall t \in T ; d \in D ; l \in L$

The MIP program (3.54) - (3.56) is also augmented by cuts (3.53).

\subsubsection{Cutting planes generation}

In this section, we describe cuts that can be added to formulation CF2 to strengthen its linear relaxation. These cuts are generated by using the Fenchel enumeration cutting planes technique (BOYD, 1994a; BOYD, 1994b). The idea is adapted from Santos et al. (2012), which employed these cuts to strengthen their column generation.

Let us consider the following notation:

- $K_{t d}=\left\{1, \cdots,\left|H_{t d}\right|\right\}$ : the set of all possible number of lessons that teacher $t$ can have on day $d$.

- $Q_{r d}=\left\{1, \cdots, \min \left\{\left|H_{t d}\right|, \widetilde{\theta}_{r}\right\}\right\}$ : the set of all possible number of lessons that requirement $r$ can have on day $d$. In which $t$ is the teacher of requirement $r$.

- $A_{k}$ : the subset of layouts in $L$ which have exactly $k$ assignments.

- $w_{t d k}$ : binary variable which indicates whether teacher $t$ has exactly $k$ lessons on day $d$.

- $v_{r d k}$ : binary variable which indicates whether requirement $r$ has exactly $k$ lessons on day $d$.

To derive valid cuts, we augment formulation CF2 by introducing new binary variables and constraints. The new variables $w_{t d k}$ (resp. $v_{r d k}$ ) are the sum of the values of all variables for teacher $t \in T$ (resp. requirement $r \in R$ ) on day $d \in D$, for which the corresponding layouts have exactly $k$ assignments, i.e.: 


$$
\begin{array}{ll}
\sum_{l \in A_{k}} y_{t d l}=w_{t d k} & \forall t \in T ; d \in D ; k \in K_{t d} \\
\sum_{l \in A_{k}} x_{r d l}=v_{r d k} & \forall r \in R ; d \in D ; k \in Q_{r d}
\end{array}
$$

Now consider each of the following valid polyhedra:

- Polyhedron $\Pi_{t}$ for lessons of a teacher $t$ :

$$
\begin{aligned}
& \sum_{d \in D} \sum_{k \in K_{t d}} k w_{t d k}=\sum_{r \in R_{t}} \widetilde{\theta}_{r} \\
& \sum_{k \in K_{t d}} w_{t d k} \leq 1 \quad \forall d \in D \\
& w_{t d k} \in\{0,1\} \quad \forall d \in D ; k \in K_{t d}
\end{aligned}
$$

Constraints (3.59) ensure that the weekly workload of teacher $t$ is met, and constraints (3.60) impose that at most one variable $w$ is active for each day.

- Polyhedron $\Pi_{r}$ for lessons of a requirement $r$ :

$$
\begin{aligned}
\sum_{d \in D} \sum_{k \in Q_{r d}} k v_{r d k}=\widetilde{\theta}_{r} & \\
\sum_{k \in Q_{r d}} v_{r d k} \leq 1 \quad & \forall d \in D \\
v_{r d k} \in\{0,1\} & \forall d \in D ; k \in Q_{r d}
\end{aligned}
$$

Constraints (3.62) ensure that the correct weekly workload of requirement $r$ is met, and constraints (3.63) impose that at most one variable $v$ is active for each day.

Let $\bar{w}$ be the fractional values of variables $w$ in the optimal solution of the linear program of augmented formulation CF2. We can easily enumerate all integer solutions $\stackrel{\circ}{w} \in \Pi_{t}$, and if there is a hyperplane which separates $\bar{w}$ from all these integer points $\stackrel{\circ}{w} \in \Pi_{t}$, then it can be checked and generated by solving the following oracle linear program $\mathscr{O}_{t}$ :

$$
\text { Maximize } z_{t}=\sum_{d \in D} \sum_{k \in K_{t d}} \bar{w}_{t d k} a_{t d k}
$$




$$
\begin{aligned}
\sum_{d \in D} \sum_{k \in K_{t d}} \stackrel{\circ}{w}_{t d k} a_{t d k} \leq 1 & \forall \stackrel{\circ}{w} \in \Pi_{t} \\
a_{t d k} \geq 0 & \forall d \in D ; k \in K_{t d}
\end{aligned}
$$

Let $\left(z_{t}^{*}, a_{t d k}^{*}\right)$ be the optimal solution of $\mathscr{O}_{t}$. If $z_{t}^{*}>1$, then the point $\bar{w}$ lies outside of $\Pi_{t}$ and

$$
\sum_{d \in D} \sum_{k \in K_{t d}} a_{t d k}^{*} w_{t d k} \leq 1
$$

is a valid violated cut. Otherwise, $\bar{w}$ belongs to $\Pi_{t}$. By adapting program (3.65) - (3.67) to manage variables $v_{r d k}$, the same idea above can be employed to identify cuts to polyhedron $\Pi_{r}$.

The described cuts are iteratively identified and inserted at the root node of the integer program of CF2. At each iteration, we solve the linear program of CF2 and run the oracle program for all teachers and requirements to check the existence of new cuts. The process stops when no cut is found.

\subsection{Column generation}

This section presents the three extended formulations for which the variables cannot be explicitly generated. All three formulations are based on model CF2 of Section 3.1.2. As these formulations have an enormous number of variables, but in the optimal solution only a small amount of the variables are greater than zero, we use a column generation algorithm to solve their linear relaxations. These solutions usually provide strong lower bounds on the optimal integer solutions of problem instances. For simplicity, we present the three formulations only for problem HSTP-A. Their extensions to manage problems HSTP-B and HSTP-C are straightforward.

\subsubsection{Formulation EF1}

In formulation CF2 of Section 3.1.2, layouts are considered per day. In this formulation, the layouts extend over the whole week and the variables are decisions if a requirement $r \in R$ or teacher $t \in T$ follows a given weekly assignment layout.

A weekly assignment layout for a fixed requirement $\stackrel{r}{r}$ is any assignment of binary values to variables $x_{r d l}^{\circ}$ in model CF2, that satisfies constraints (3.33) and (3.35).

A weekly assignment layout for a fixed teacher $t$ is any assignment of binary values to variables $y_{t d l}$ in model $\mathrm{CF} 2$, that satisfies constraints (3.36) to (3.38) and ensures that his/her workload is completely scheduled. 
In other words, a weekly assignment layout is a binary vector of size $|D| \cdot|H|$. For example, consider a toy instance with $|D|=3$ days and $|H|=5$ periods per day. The layout "00000 $0001100000 "$ specifies that a given requirement (or teacher) has two consecutive lessons at the last two periods of the second day, and the layout "10001 0100100000 " specifies that a teacher has three idle periods on the first day and two at the second. Table 8 describes the notation used in formulation EF1, and the mixed-integer program can thus be written as:

Table 8 - Notation used in formulation EF1.

\begin{tabular}{ll}
\hline Notation & Definition \\
\hline Sets & the set of all possible assignment layouts that a requirement $r$ can follow during the week. \\
$L_{r}$ & the set of all possible assignment layouts that a teacher $t$ can follow during the week. \\
$L_{t}$ & \\
Parameters & the cost of violating requisites 5 to 7 in the $i^{t h}$ weekly layout of requirement $r$. \\
$f_{r i}$ & the cost of breaking requisites 8 and 9 in the $j^{t h}$ weekly layout of teacher $t$. \\
$g_{t j}$ & constant that indicates whether requirement $r$ is assigned to period $h$ of day $d$ in the $i^{t h}$ \\
$\bar{x}_{r i}^{d h} \in\{0,1\}$ & weekly layout. \\
$\bar{y}_{t j}^{d h} \in\{0,1\}$ & constant that indicates whether teacher $t$ is assigned to period $h$ of day $d$ in the $j^{t h}$ weekly \\
$\widetilde{\phi}_{l} \in \mathbb{N}$ & layout. \\
Decision variables & the number of days that have at least one assignment in a weekly layout $l$. \\
$\lambda_{r i} \in\{0,1\}$ & indicates whether requirement $r$ follows the $i^{t h}$ weekly layout. \\
$\chi_{t j} \in\{0,1\}$ & indicates whether teacher $t$ follows the $j^{t h}$ weekly layout. \\
Auxiliary variables & \\
$\widehat{\beta} \in \mathbb{N}$ & the greatest value among all teachers' extra working days. \\
\hline
\end{tabular}

Minimize $\sum_{r \in R} \sum_{i \in L_{r}} f_{r i} \lambda_{r i}+\sum_{t \in T} \sum_{j \in L_{t}} g_{t j} \chi_{t j}+\alpha_{10} \widehat{\beta}$

Subject to:

$$
\begin{array}{rlrl}
\left(\xi_{r}^{1}\right) \quad \sum_{i \in L_{r}} \lambda_{r i}=1 & \forall r \in R \\
\left(\xi_{t}^{2}\right) \quad \sum_{j \in L_{t}} \chi_{t j}=1 & \forall t \in T \\
\left(\xi_{c d h}^{3}\right) \quad \sum_{r \in R_{c}} \sum_{i \in L_{r}} \bar{x}_{r i}^{d h} \lambda_{r i}=1 & \forall c \in C ; d \in D ; h \in H \\
\left(\xi_{t d h}^{4}\right) & \sum_{r \in R_{t}} \sum_{i \in L_{r}} \bar{x}_{r i}^{d h} \lambda_{r i}-\sum_{j \in L_{t}} \bar{y}_{t j}^{d h} \chi_{t j}=0 & \forall t \in T ; d \in D ; h \in H \\
\left(\xi_{t}^{5}\right) & \widehat{\beta}-\sum_{j \in L_{t}} \widetilde{\phi}_{j} \chi_{t j} \geq-\widetilde{d}_{t} & \forall t \in T
\end{array}
$$




$$
\begin{array}{rr}
\widehat{\beta} \geq 0 & \\
\lambda_{r i} \in\{0,1\} & \forall r \in R ; i \in L_{r} \\
\chi_{t j} \in\{0,1\} & \forall t \in T ; j \in L_{t}
\end{array}
$$

The objective function (3.69) minimizes the cost of weekly layouts related to violating requisites 5 to 9 , plus the cost of breaking requisite 10. Constraints (3.70) and (3.71) ensure that only one weekly layout is selected for each requirement and teacher. Constraints (3.72) avoid clashes in classes' schedules. Constraints (3.73) link layouts of teachers and requirements. The last constraints (3.74) model the balancing on teachers' extra working days.

For practical size instances, formulation EF1 usually has a huge number of variables that are hard to generate explicitly. Even if the explicit writing of the problem is possible, it will result in MIP programs that usually cannot be tackled by actual solvers. On the other hand, as the optimal solutions are defined by small subsets of such variables, we can use column generation (DESAULNIERS; DESROSIERS; SOLOMON, 2005) as an effective tool to solve the associated relaxed problem. The linear relaxation of EF1 usually provides tight lower bounds on the optimal solutions of its original MIP program.

Our column generation procedure works as follows. The master problem is a linear relaxation of program (3.69) - (3.77), and the columns are generated via two types of pricing subproblems. The subproblems defined by $\mathscr{P}_{r}$ (Section 3.2.1.1) generate the columns with reduced costs associated with weekly layouts of requirements and the subproblems $\mathscr{P}_{t}$ (Section 3.2.1.2) generate the columns with reduced costs associated with weekly layouts of teachers. The initial master program contains a reduced number of artificial columns, to which we assign high costs. At each iteration, every subproblem $\mathscr{P}_{r}$ and $\mathscr{P}_{t}$ is solved to obtain the columns that have the smallest reduced costs, for requirements and teachers, based on the dual values of the last master program solution. The new columns are inserted into the master program, which is solved again, and the process continues until there is no column with negative cost.

\subsubsection{Subproblems $\mathscr{P}_{r}$}

There are $|R|$ pricing subproblems, one for each requirement $r \in R$. Each subproblem $\mathscr{P}_{r}$ generates columns (weekly layouts) with costs related to requisites 5 to 7 . Table 9 defines the notation used in the formulation of subproblem $\mathscr{P}_{r}$ and the mixed-integer program can thus be written as: 
Table 9 - Notation used in subproblem $\mathscr{P}_{r}$.

\begin{tabular}{ll}
\hline Notation & Definition \\
\hline Sets & the set of all possible assignment layouts that a requirement $r$ can follow in a day. \\
& \\
Parameters & the number of assignments in layout $l \in L$. \\
$\widetilde{a}_{l} \in \mathbb{N}$ & the number of lessons that exceeds the daily limit $\widetilde{\delta}_{r}$ in layout $l$ for a requirement $r$. \\
$\widetilde{e}_{r l} \in \mathbb{N}$ & the number of holes in layout $l$ for requirements. \\
$\widetilde{h}_{l} \in \mathbb{N}$ & the number of double lessons in layout $l$. \\
$\widetilde{\omega}_{l} \in \mathbb{N}$ & indicates whether layout $l$ has an assignment at period $h$. \\
$\widetilde{\mu}_{l h} \in\{0,1\}$ & the class associated with requirement $r$. \\
$\rho(r)$ & the teacher associated with requirement $r$. \\
$\sigma(r)$ & \\
Decision variables & \\
$x_{d l} \in\{0,1\}$ & indicates whether requirement $r$ follows layout $l$ on day $d$. \\
& \\
Auxiliary variables & \\
$\widehat{\pi} \in \mathbb{N}$ & the number of unmet weekly double lessons of requirement $r$. \\
\hline
\end{tabular}

$$
\begin{array}{ll}
\text { Minimize } & \bar{c}_{r}=\sum_{d \in D} \sum_{l \in L}\left(\alpha_{5} \widetilde{e}_{r l}+\alpha_{6} \widetilde{h}_{l}\right) x_{d l}+\alpha_{7} \widehat{\pi} \\
& -\xi_{r}^{1}-\sum_{d \in D} \sum_{h \in H} \xi_{\rho(r) d h}^{3} \sum_{l \in L} \widetilde{\mu}_{l h} x_{d l}-\sum_{d \in D} \sum_{h \in H} \xi_{\sigma(r) d h}^{4} \sum_{l \in L} \widetilde{\mu}_{l h} x_{d l}
\end{array}
$$

Subject to:

$$
\begin{gathered}
\sum_{d \in D} \sum_{l \in L} \widetilde{a}_{l} x_{d l}=\widetilde{\theta}_{r} \\
\sum_{l \in L} x_{d l}=1 \quad \forall d \in D \\
\widehat{\pi} \geq \tilde{\pi}_{r}-\sum_{d \in D} \sum_{l \in L} \widetilde{\omega}_{l} x_{d l} \\
\widehat{\pi} \geq 0 \\
x_{d l} \in\{0,1\} \quad \forall d \in D ; l \in L
\end{gathered}
$$

The objective function (3.78) is the reduced cost $\bar{c}_{r}$ involving the dual values $\xi_{r}^{1}, \xi_{c d h}^{3}$ and $\xi_{t d h}^{4}$ associated with constraints (3.70), (3.72) and (3.73) of the master problem (3.69) (3.77). Constraints (3.79) ensure that the required number of weekly lessons of requirement $r$ is scheduled. Constraints (3.80) ensure that requirement $r$ follows only one layout per day, and constraints (3.81) compute its number of unmet double lessons.

\subsubsection{Subproblems $\mathscr{P}_{t}$}

There are $|T|$ pricing subproblems, one for each teacher $t \in T$. Each subproblem $\mathscr{P}_{t}$ generates columns (weekly layouts) with costs related to requisites 8 and 9 . Table 10 defines the 
notation used in the formulation of subproblem $\mathscr{P}_{t}$ and the mixed-integer program can thus be written as:

Table 10 - Notation used in subproblem $\mathscr{P}_{t}$.

\begin{tabular}{ll}
\hline Notation & Definition \\
\hline Sets & the set of all possible assignment layouts that a teacher $t$ can follow in a day. \\
$L$ & \\
Parameters & the number of idle periods in layout $l \in L$ for teacher $t$ on day $d$. \\
$\widetilde{i}_{d l} \in \mathbb{N}$ & indicates whether the number of assignments in layout $l$ is greater than zero. \\
$\widetilde{\phi}_{l} \in\{0,1\}$ & \\
\begin{tabular}{ll} 
Decision variables & \\
$y_{d l} \in\{0,1\}$ & indicates whether teacher $t$ follows layout $l$ on day $d$. \\
\hline
\end{tabular}
\end{tabular}

$$
\begin{array}{ll}
\text { Minimize } & \bar{c}_{t}=\sum_{d \in D} \sum_{l \in L}\left(\alpha_{8}{\widetilde{i_{t}}}_{t l}+\alpha_{9} \widetilde{\phi}_{l}\right) y_{d l} \\
& -\xi_{t}^{2}+\sum_{d \in D} \sum_{h \in H} \xi_{t d h}^{4} \sum_{l \in L} \widetilde{\mu}_{l h} y_{d l}+\xi_{t}^{5} \sum_{d \in D} \sum_{l \in L} \widetilde{\phi}_{l} y_{d l}
\end{array}
$$

Subject to:

$$
\begin{aligned}
& \sum_{d \in D} \sum_{l \in L} \widetilde{a}_{l} y_{d l}=\sum_{r \in R_{t}} \widetilde{\theta}_{r} \\
& \sum_{l \in L} y_{d l}=1 \quad \forall d \in D \\
& \sum_{d \in D} \sum_{l \in L} \widetilde{\phi}_{l} y_{d l} \geq \widetilde{d}_{t} \\
& y_{d l} \in\{0,1\} \quad \forall d \in D ; l \in L
\end{aligned}
$$

The objective function (3.84) is the reduced cost $\bar{c}_{t}$ involving the dual values $\xi_{t}^{2}, \xi_{t d h}^{4}$ and $\xi_{t}^{5}$ associated with constraints (3.71), (3.73) and (3.74) of the master problem (3.69) - (3.77). Constraints (3.85) ensure that the weekly workload of teacher $t$ is scheduled. Constraints (3.86) ensure that teacher $t$ follows only one layout per day. The last constraints (3.87) are the cuts to ensure that teacher $t$ does not work less than the minimum number of working days $\widetilde{d}_{t}$ (SOUZA, 2000). To avoid violations in teachers' unavailable periods, we preprocess the variables as follows.

$$
y_{d l}=0 \quad \forall d \in D ; l \in L ; h \in H \backslash H_{t d} ; \widetilde{\mu}_{l h}>0
$$




\subsubsection{Formulation EF2}

In formulation EF1, there are columns for requirements and teachers. In this formulation, columns are generated only for teachers. A column contains the weekly assignment layouts of all requirements belonging to a given teacher. The variables are decisions if a teacher $t \in T$ follows

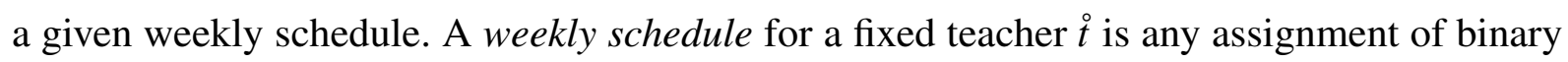
values to variables $y_{t d l}$ and $x_{r d l}$ (for all $r \in R_{t}$ ) in model CF2, that satisfies constraints (3.33), (3.35) to (3.38), and (3.45). Table 11 describes the notation used in formulation EF2 and the mixed-integer program can thus be written as:

Table 11 - Notation used in formulation EF2.

\begin{tabular}{ll}
\hline Notation & Definition \\
\hline Sets & the set of all possible weekly schedules that a teacher $t \in T$ can follow. \\
$S_{t}$ & the set of teachers who teach to class $c \in C$. \\
$T_{c}$ & \\
Parameters & the cost of violating requisites 5 to 9 in the $i^{t h}$ weekly schedule of teacher $t$. \\
$f_{t i}$ & constant that indicates whether teacher $t$ teaches to class $c$ in schedule $i \in S_{t}$ at period \\
$\bar{x}_{t i}^{c h} \in\{0,1\}$ & $\begin{array}{l}h \text { of day } d . \\
\text { the number of worked days for teacher } t \text { in schedule } i \in S_{t} .\end{array}$ \\
$\widetilde{\phi}_{t i} \in \mathbb{N}$ & \\
$\begin{array}{l}\text { Decision variables } \\
\lambda_{t i} \in\{0,1\}\end{array}$ & indicates whether teacher $t$ follows the weekly schedule $i \in S_{t}$. \\
$\begin{array}{l}\text { Auxiliary variables } \\
\widehat{\beta} \in \mathbb{N}\end{array}$ & the greatest value among all teachers' extra working days. \\
\hline
\end{tabular}

Minimize $\sum_{t \in T} \sum_{i \in S_{t}} f_{t i} \lambda_{t i}+\alpha_{10} \widehat{\beta}$

Subject to:

$$
\begin{array}{rlr}
\left(\xi_{t}{ }^{1}\right) \quad \sum_{i \in S_{t}} \lambda_{t i}=1 & \forall t \in T \\
\left(\xi_{c d h}^{2}\right) \quad \sum_{t \in T_{c}} \sum_{i \in S_{t}} \bar{x}_{t i}^{c d h} \lambda_{t i}=1 & \forall c \in C ; d \in D ; h \in H \\
\left(\xi_{t}^{3}\right) \quad \widehat{\beta}-\sum_{i \in S_{t}} \widetilde{\phi}_{t i} \lambda_{t i} \geq-\widetilde{d}_{t} & \forall t \in T \\
\widehat{\beta} \geq 0 & \\
\lambda_{t i} \in\{0,1\} & \forall t \in T ; i \in S_{t}
\end{array}
$$

The objective function (3.90) minimizes the cost of weekly schedules related to violating requisites 5 to 9 , plus the cost of breaking the requisite 10. Constraints (3.91) ensure that only 
one weekly schedule is selected for each teacher. Constraints (3.92) avoid clashes in classes' schedules, and the last constraints (3.93) model the balancing on teachers' extra working days.

For this formulation, the master problem of our column generation algorithm is a linear relaxation of program (3.90) - (3.95), and the columns are generated via $|T|$ pricing subproblems $\mathscr{Q}_{t}$, described in Section 3.2.2.1, which select the most attractive weekly schedules (columns) for teachers. The master program starts with a reduced number of artificial columns, with high costs, and at each iteration, every subproblem $\mathscr{Q}_{t}$ is solved to obtain the columns that have the smallest reduced costs for teachers.

\subsubsection{Subproblems $\mathscr{Q}_{t}$}

Each pricing subproblem $\mathscr{Q}_{t}$ generates columns (weekly schedules) with costs related to requisites 5 to 9 . Table 12 defines the notation used in the formulation of subproblem $\mathscr{Q}_{t}$ and the mixed-integer program can thus be written as:

Table 12 - Notation used in subproblem $\mathscr{Q}_{t}$.

\begin{tabular}{|c|c|}
\hline Notation & Definition \\
\hline \multicolumn{2}{|l|}{ Sets } \\
\hline$L$ & $\begin{array}{l}\text { the set of all possible assignment layouts that a teacher } t \text { and requirements } r \in R_{t} \text { can } \\
\text { follow in a day. }\end{array}$ \\
\hline$C_{t}$ & the set of classes taught by teacher $t$. \\
\hline$R_{c t}$ & the set of requirements belonging to class $c$ which are taught by teacher $t$. \\
\hline \multicolumn{2}{|l|}{ Parameters } \\
\hline$\widetilde{a}_{l} \in \mathbb{N}$ & the number of assignments in layout $l \in L$. \\
\hline$\widetilde{\omega}_{l} \in \mathbb{N}$ & the number of consecutive double lessons in layout $l$. \\
\hline$\widetilde{e}_{r l} \in \mathbb{N}$ & $\begin{array}{l}\text { the number of lessons that exceeds the daily limit } \widetilde{\delta}_{r} \text { in layout } l \text { for a requirement } \\
r \in R_{t} \text {. }\end{array}$ \\
\hline$\widetilde{h}_{l} \in \mathbb{N}$ & the number of holes in layout $l$ for requirements. \\
\hline$\widetilde{\mu}_{l h} \in\{0,1\}$ & indicates whether layout $l$ has an assignment at period $h$. \\
\hline$\widetilde{i}_{t d l} \in \mathbb{N}$ & the number of idle periods in layout $l$ for teacher $t$ on day $d$. \\
\hline$\widetilde{\phi}_{l} \in\{0,1\}$ & indicates whether the number of assignments in layout $l$ is greater than zero. \\
\hline \multicolumn{2}{|c|}{ Decision variables } \\
\hline$y_{d l} \in\{0,1\}$ & indicates whether teacher $t$ follows layout $l$ on day $d$. \\
\hline$x_{r d l} \in\{0,1\}$ & indicates whether requirement $r \in R_{t}$ follows layout $l$ on day $d$. \\
\hline \multicolumn{2}{|c|}{ Auxiliary variables } \\
\hline$\widehat{\pi}_{r} \in \mathbb{N}$ & the number of unmet weekly double lessons of requirement $r$. \\
\hline
\end{tabular}

$$
\begin{array}{cl}
\text { Minimize } & \bar{c}_{t}=\sum_{r \in R_{t}} \sum_{d \in D} \sum_{l \in L}\left(\alpha_{5} \widetilde{e}_{r l}+\alpha_{6} \widetilde{h}_{l}\right) x_{r d l}+\alpha_{7} \sum_{r \in R_{t}} \widehat{\pi}_{r}+\sum_{d \in D} \sum_{l \in L}\left(\alpha_{8} \tilde{i}_{t d l}+\alpha_{9} \widetilde{\phi}_{l}\right) y_{d l} \\
& -\xi_{t}^{1}-\sum_{c \in C_{t}} \sum_{d \in D} \sum_{h \in H} \xi_{c d h}^{2} \sum_{r \in R_{c t}} \sum_{l \in L} \widetilde{\mu}_{l h} x_{r d l}+\xi_{t}^{3} \sum_{d \in D} \sum_{l \in L} \widetilde{\phi}_{l} y_{d l}
\end{array}
$$

Subject to: 


$$
\begin{aligned}
\sum_{d \in D} \sum_{l \in L} \widetilde{a}_{l} x_{r d l}=\widetilde{\theta}_{r} & \forall r \in R_{t} \\
\sum_{r \in R_{c t}} \sum_{l \in L} \widetilde{\mu}_{l h} x_{r d l}=1 & \forall c \in C_{t} ; d \in D ; h \in H \\
\sum_{l \in L} x_{r d l}=1 & \forall r \in R_{t} ; d \in D \\
\sum_{l \in L} y_{d l}=1 & \forall d \in D \\
\sum_{r \in R_{t}} \sum_{l \in L} \widetilde{\mu}_{l h} x_{r d l}=\sum_{l \in L} \widetilde{\mu}_{l h} y_{d l} & \forall d \in D ; h \in H \\
\widehat{\pi}_{r} \geq \widetilde{\pi}_{r}-\sum_{d \in D} \sum_{l \in L} \widetilde{\omega}_{l} x_{r d l} & \forall r \in R_{t} \\
\sum_{d \in D} \sum_{l \in L} \widetilde{\phi}_{l} y_{d l} \geq \widetilde{d}_{t} & \\
\widehat{\pi}_{r} \geq 0 & \forall r \in R_{t} \\
x_{r d l} \in\{0,1\} & \forall r \in R_{t} ; d \in D ; l \in L \\
y_{d l} \in\{0,1\} & \forall d \in D ; l \in L
\end{aligned}
$$

The objective function (3.96) is the reduced cost $\bar{c}_{t}$ involving the dual values $\xi_{t}^{1}, \xi_{c d h}^{2}$ and $\xi_{t}^{3}$ associated with constraints (3.91) to (3.93) of the master problem (3.90) - (3.95). Constraints (3.97) ensure that the required number of weekly lessons is scheduled for each requirement of a teacher $t$. Constraints (3.98) avoid clashes in classes' schedules. We also need these constraints in the subproblems to avoid cases in which a teacher teaches to the same class in two different requirements $r_{1} \in R$ and $r_{2} \in R$. A frequent case is a teacher $t$ who teaches mathematics (represented by $r_{1}$ ) and physics (represented by $r_{2}$ ) to the same class. Constraints (3.99) and (3.100) ensure that teacher $t$ and his/her requirements $r \in R_{t}$ follow only one layout per day. The linking constraints (3.101) unsure that the daily schedules of teacher $t$ match his/her requirements' schedules. Constraints (3.102) compute the number of unmet double lessons for requirements. The last constraints (3.103) are the cuts to ensure that teacher $t$ does not work less than the minimum number of working days $\widetilde{d}_{t}$ (SOUZA, 2000).

To avoid violating teachers' unavailable periods, we preprocess the variables as follows.

$$
\begin{aligned}
y_{d l}=0 & \forall d \in D ; l \in L ; h \in H \backslash H_{t d} ; \widetilde{\mu}_{l h}>0 \\
x_{r d l}=0 & \forall r \in R_{t} ; d \in D ; l \in L ; h \in H \backslash H_{t d} ; \widetilde{\mu}_{l h}>0
\end{aligned}
$$

\subsubsection{Formulation EF3}

In this formulation, variables are decisions if a weekday $d \in D$ follows a given schedule.

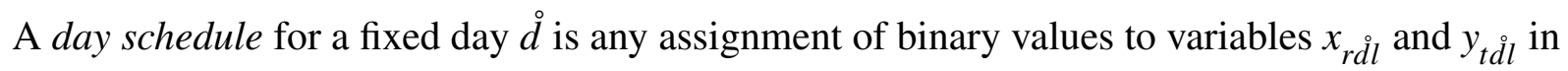


model CF2, that satisfies constraints (3.34) to (3.38) and (3.45). Table 13 describes the notation used in formulation EF3 and the mixed-integer program can thus be written as:

Table 13 - Notation used in formulation EF3.

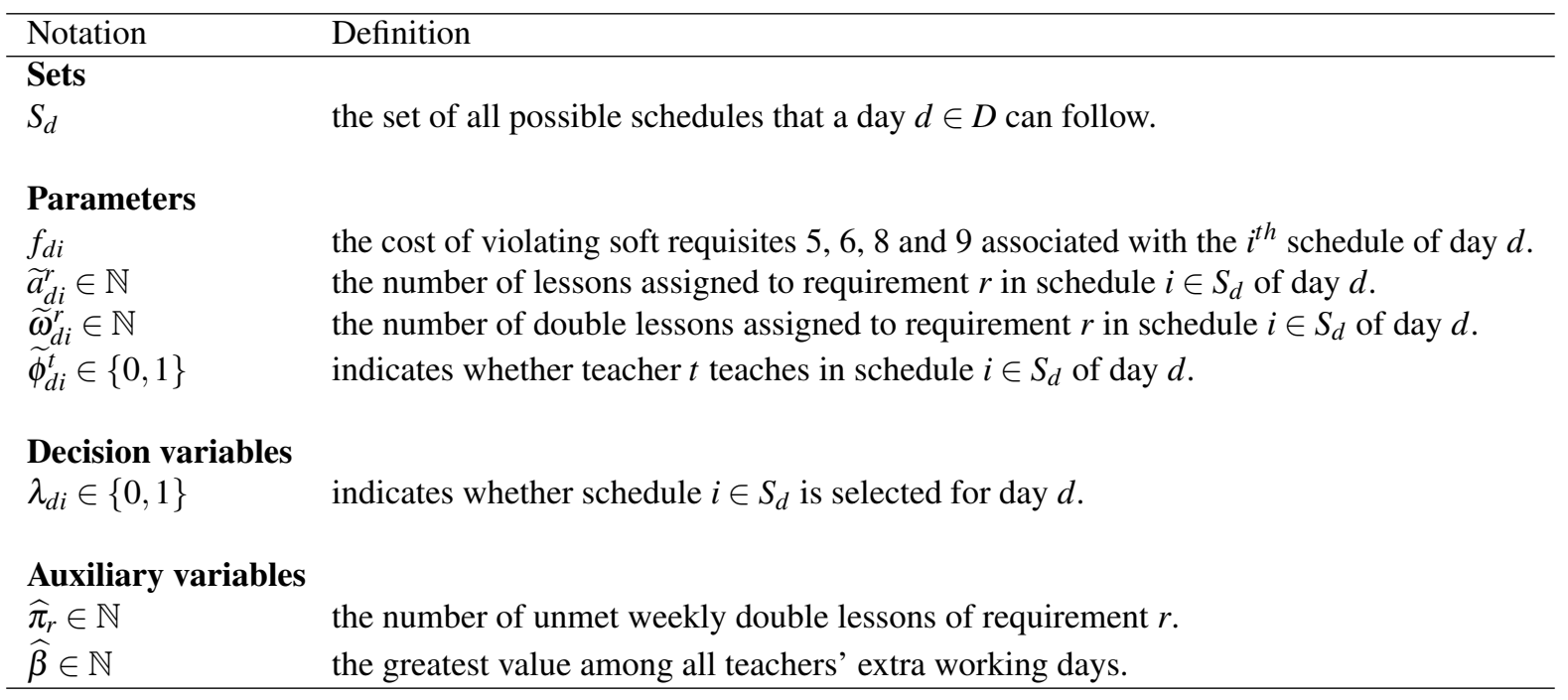

Minimize $\sum_{d \in D} \sum_{i \in S_{d}} f_{d i} \lambda_{d i}+\alpha_{7} \sum_{r \in R} \widehat{\pi}_{r}+\alpha_{10} \widehat{\beta}$

Subject to:

$$
\begin{aligned}
\left(\xi_{d}^{1}\right) \quad \sum_{i \in S_{d}} \lambda_{d i}=1 & \forall d \in D \\
\left(\xi_{r}^{2}\right) \quad \sum_{d \in D} \sum_{i \in S_{d}} \widetilde{a}_{d i}^{r} \lambda_{d i}=\widetilde{\theta}_{r} & \forall r \in R \\
\widehat{\pi}_{r}+\sum_{d \in D} \sum_{i \in S_{d}} \widetilde{\omega}_{d i}^{r} \lambda_{d i} \geq \widetilde{\pi}_{r} & \forall r \in R \\
\left(\xi_{t}^{4}\right) \quad \widehat{\beta}-\sum_{d \in D} \sum_{i \in S_{d}} \widetilde{\phi}_{d i}^{t} \lambda_{d i} \geq-\widetilde{d}_{t} & \forall t \in T \\
\sum_{d \in D} \sum_{i \in S_{d}} \widetilde{\phi}_{d i}^{t} \lambda_{d i} \geq \widetilde{d}_{t} & \forall t \in T \\
\widehat{\pi}_{r} \geq 0 & \forall r \in R \\
\widehat{\beta} \geq 0 & \\
\lambda_{d i} \in\{0,1\} & \forall d \in D ; i \in S_{d}
\end{aligned}
$$

The objective function (3.109) minimizes the cost of day schedules that violate requisites $5,6,8$ and 9, plus the cost of infringing requisites 7 and 10. Constraints (3.110) ensure that only one schedule is selected for each day. Constraints (3.111) ensure that the required number of weekly lessons is scheduled for each requirement. Constraints (3.112) compute the number of 
unmet double lessons for requirements. Constraints (3.113) model the balancing on teachers' extra working days. The last constraints (3.114) are the cuts to ensure that each teacher $t$ does not work less than the minimum number of working days $\widetilde{d}_{t}$ (SOUZA, 2000).

For this formulation, the master problem of our column generation algorithm is a linear relaxation of program (3.109) - (3.117), and the columns are generated via $|D|$ pricing subproblems $\mathscr{B}_{d}$ which are described in Section 3.2.3.1. The master program starts with a reduced number of artificial columns, containing high costs, and at each iteration, every subproblem $\mathscr{B}_{d}$ is solved to obtain the columns that have the smallest reduced costs for days.

\subsubsection{Subproblem $\mathscr{B}_{d}$}

Each pricing subproblem $\mathscr{B}_{d}$ generates columns (day schedules) with costs related to requisites 5, 6, 8 and 9. Table 14 defines the notation used in the formulation of subproblem $\mathscr{B}_{d}$ and the mixed-integer program can thus be written as:

Table 14 - Notation used in subproblem $\mathscr{B}_{d}$.

\begin{tabular}{ll}
\hline Notation & Definition \\
\hline$L$ & $\begin{array}{l}\text { the set of all possible assignment layouts that a requirement } r \text { (or teacher } t \text { ) can follow } \\
\text { on day } d .\end{array}$ \\
Parameters & \\
$\widetilde{a}_{l} \in \mathbb{N}$ & the number of assignments in layout $l \in L$. \\
$\widetilde{\omega}_{l} \in \mathbb{N}$ & the number of consecutive double lessons in layout $l$. \\
$\widetilde{e}_{r l} \in \mathbb{N}$ & the number of lessons that exceeds the daily limit $\widetilde{\delta}_{r}$ in layout $l$ for a requirement $r$. \\
$\widetilde{h}_{l} \in \mathbb{N}$ & the number of holes in layout $l$ for requirements. \\
$\widetilde{\mu}_{l h} \in\{0,1\}$ & indicates whether layout $l$ has an assignment at period $h$. \\
$\widetilde{i}_{t d l} \in \mathbb{N}$ & the number of idle periods in layout $l$ for teacher $t$ on day $d$. \\
$\widetilde{\phi}_{l} \in\{0,1\}$ & indicates whether the number of assignments in layout $l$ is greater than zero. \\
Decision variables & \\
$x_{r l} \in\{0,1\}$ & indicates whether requirement $r$ follows layout $l$ on day $d$. \\
$y_{t l} \in\{0,1\}$ & indicates whether teacher $t$ follows layout $l$ on day $d$.
\end{tabular}

$$
\begin{aligned}
\text { Minimize } & \bar{c}_{d}=\sum_{r \in R} \sum_{l \in L}\left(\alpha_{5} \widetilde{e}_{r l}+\alpha_{6} \widetilde{h}_{l}\right) x_{r l}+\sum_{t \in T} \sum_{l \in L}\left(\alpha_{8} \widetilde{i}_{t d l}+\alpha_{9} \widetilde{\phi}_{l}\right) y_{t l} \\
& -\xi_{d}^{1}-\sum_{r \in R} \sum_{l \in L}\left(\xi_{r}^{2} \widetilde{a}_{l}+\xi_{r}^{3} \widetilde{\omega}_{l}\right) x_{r l}+\sum_{t \in T} \sum_{l \in L}\left(\xi_{t}^{4}-\xi_{t}^{5}\right) \widetilde{\phi}_{l} y_{t l}
\end{aligned}
$$

Subject to:

$$
\begin{array}{ll}
\sum_{l \in L} x_{r l}=1 & \forall r \in R \\
\sum_{l \in L} y_{t l}=1 & \forall t \in T
\end{array}
$$




$$
\begin{aligned}
\sum_{r \in R_{c}} \sum_{l \in L} \widetilde{\mu}_{l h} x_{r l}=1 & \forall c \in C ; h \in H \\
\sum_{r \in R_{t}} \sum_{l \in L} \widetilde{\mu}_{l h} x_{r l}=\sum_{l \in L} \widetilde{\mu}_{l h} y_{t l} & \forall t \in T ; h \in H \\
x_{r l} \in\{0,1\} & \forall r \in R ; l \in L \\
y_{t l} \in\{0,1\} & \forall t \in T ; l \in L
\end{aligned}
$$

The objective function (3.118) is the reduced cost $\bar{c}_{d}$ involving the dual values $\xi_{d}^{1}, \xi_{r}^{2}, \xi_{r}^{3}$, $\xi_{t}^{4}$ and $\xi_{t}^{5}$ associated with constraints (3.110) to (3.114) of the master problem (3.109) - (3.117). Constraints (3.119) and (3.120) ensure that each requirement and teacher follows only one layout per day. Constraints (3.121) avoid clashes in classes' schedules. The last constraints (3.122) link layouts of teachers and requirements on day $d$.

To avoid violations in teachers' unavailable periods, we preprocess the variables as follows.

$$
\begin{array}{ll}
y_{t l}=0 & \forall t \in T ; l \in L ; h \in H \backslash H_{t d} ; \widetilde{\mu}_{l h}>0 \\
x_{r l}=0 & \forall t \in T ; r \in R_{t} ; l \in L ; h \in H \backslash H_{t d} ; \widetilde{\mu}_{l h}>0
\end{array}
$$

\subsection{Computational experiments}

In this section, we report three experiments in order to assess the proposed formulations.

Section 3.3.1: we analyze formulations CF1, CF2, EF1, EF2, and EF3 applied to problem HSTP-A.

Section 3.3.2: the best compact formulation and the best extended formulation are adjusted to problem HSTP-B and the results are compared with literature results.

Section 3.3.3: the best formulations are adjusted and applied to problem HSTP-C.

The programs of all formulations were coded in C++ using the Concert Technology Libraries from CPLEX 12.6 and compiled with GNU Compiler Collection 4.4.7. The experiments were carried out on a server with 2 CPU Intel Xeon E5-2680v2 $(2.8 \mathrm{GHz})$ and $128 \mathrm{~GB}$ of RAM, running the operating system Red Hat Enterprise Linux Server 6.5.

\subsubsection{Results for problem HSTP-A}

This section evaluates the proposed formulations on problem HSTP-A. We used 34 instances collected from Brazilian high schools (SAVINIEC; CONSTANTINO, 2017). Table 15 
Table 15 - Features of the 34 real instances from Brazilian high schools.

\begin{tabular}{|c|c|c|c|c|c|c|c|}
\hline ID & Instance & $|C|$ & $|T|$ & $|D|$ & $|H|$ & $\sum_{r \in R} \widetilde{\theta}_{r}$ & $\sum_{r \in R} \tilde{\pi}_{r}$ \\
\hline 1 & CL-CEASD-2008-V-A & 12 & 27 & 5 & 5 & 300 & 132 \\
\hline 2 & CL-CEASD-2008-V-B & 12 & 27 & 5 & 5 & 300 & 132 \\
\hline 3 & CL-CECL-2011-M-A & 13 & 31 & 5 & 5 & 325 & 144 \\
\hline 4 & CL-CECL-2011-M-B & 13 & 31 & 5 & 5 & 325 & 143 \\
\hline 5 & CL-CECL-2011-N-A & 9 & 28 & 5 & 5 & 225 & 107 \\
\hline 6 & CL-CECL-2011-V-A & 14 & 29 & 5 & 5 & 350 & 164 \\
\hline 7 & CM-CECM-2011-M & 20 & 51 & 5 & 5 & 500 & 234 \\
\hline 8 & CM-CECM-2011-N & 8 & 30 & 5 & 5 & 200 & 96 \\
\hline 9 & CM-CЕCM-2011-V & 13 & 34 & 5 & 5 & 325 & 142 \\
\hline 10 & CM-CEDB-2010-N & 5 & 17 & 5 & 5 & 125 & 60 \\
\hline 11 & CM-CEUP-2008-V & 16 & 35 & 5 & 5 & 400 & 192 \\
\hline 12 & CM-CEUP-2011-M & 16 & 38 & 5 & 5 & 400 & 192 \\
\hline 13 & CM-CEUP-2011-N & 3 & 15 & 5 & 5 & 75 & 36 \\
\hline 14 & CM-CEUP-2011-V & 16 & 34 & 5 & 5 & 400 & 169 \\
\hline 15 & FA-EEF-2011-M & 4 & 12 & 5 & 5 & 100 & 42 \\
\hline 16 & JNS-CEDPII-2011-M & 8 & 19 & 5 & 5 & 200 & 85 \\
\hline 17 & JNS-CEDPII-2011-V & 7 & 21 & 5 & 5 & 175 & 73 \\
\hline 18 & JNS-CEJXXIII-2011-M & 5 & 18 & 5 & 5 & 125 & 60 \\
\hline 19 & JNS-CEJXXIII-2011-N & 4 & 15 & 5 & 5 & 100 & 48 \\
\hline 20 & JNS-CEJXXIII-2011-V & 5 & 18 & 5 & 5 & 125 & 60 \\
\hline 21 & MGA-CEDC-2011-M & 19 & 37 & 5 & 5 & 475 & 210 \\
\hline 22 & MGA-CEDC-2011-V & 12 & 31 & 5 & 5 & 300 & 131 \\
\hline 23 & MGA-CEGV-2011-M & 31 & 62 & 5 & 5 & 775 & 352 \\
\hline 24 & MGA-CEGV-2011-V & 32 & 75 & 5 & 5 & 800 & 357 \\
\hline 25 & MGA-CEJXXIII-2010-V & 16 & 35 & 5 & 5 & 400 & 192 \\
\hline 26 & MGA-CEVB-2011-M & 10 & 21 & 5 & 5 & 250 & 108 \\
\hline 27 & MGA-CEVB-2011-V & 9 & 20 & 5 & 5 & 225 & 97 \\
\hline 28 & NE-CESVP-2011-M-A & 18 & 45 & 5 & 5 & 450 & 212 \\
\hline 29 & NE-CESVP-2011-M-B & 18 & 44 & 5 & 5 & 450 & 212 \\
\hline 30 & NE-CESVP-2011-M-C & 18 & 45 & 5 & 5 & 450 & 211 \\
\hline 31 & NE-CESVP-2011-M-D & 18 & 45 & 5 & 5 & 450 & 211 \\
\hline 32 & NE-CESVP-2011-V-A & 16 & 44 & 5 & 5 & 400 & 183 \\
\hline 33 & NE-CESVP-2011-V-B & 16 & 43 & 5 & 5 & 400 & 184 \\
\hline 34 & NE-CESVP-2011-V-C & 16 & 43 & 5 & 5 & 400 & 182 \\
\hline
\end{tabular}

presents the main features of these instances. The columns present the number of classes $(|C|)$, teachers $(|T|)$, days $(|D|)$, periods per day $(|H|)$, the total number of weekly lessons $\left(\sum_{r \in R} \widetilde{\theta}_{r}\right)$ and the total number of consecutive double lessons $\left(\sum_{r \in R} \tilde{\pi}_{r}\right)$ required for each instance, respectively. The following weighting parameters are used in the objective function for these instances: $\alpha_{5}=100, \alpha_{6}=25, \alpha_{7}=\alpha_{8}=\alpha_{9}=\alpha_{10}=10$. These parameter values were originated from the school's preferences for attending the considered list of soft requisites.

\subsubsection{Comparing the linear programs of formulations CF1 and CF2}

This section compares the linear relaxation of formulations $\mathrm{CF} 1$ and $\mathrm{CF} 2$. Both programs were run with CPLEX's default settings. The results are shown in Table 16. The table presents for each formulation, the execution time (Time) and the value of the linear program $(L P)$ on the tested instances. The columns $\left(L P 2_{A}\right)$ and $\left(L P 2_{B}\right)$ are the $L P$ values of $C F 2$ before and after the addition of the Fenchel cuts described in Section 3.1.2.4, respectively. The gaps presented 
on columns labeled with $\left(G a p_{\#}\right)$ are calculated as $G a p_{\#}=100 \cdot\left(L P 2_{\#}-L P 1\right) \div L P 2_{\#}$, for \# representing $\mathrm{A}$ or $\mathrm{B}$. The results of Table 16 show that $\mathrm{CF} 2$ has better linear relaxation than $\mathrm{CF} 1$ and its linear program also runs faster. On average, the LP of CF2 is $21.09 \%$ better than the LP of CF1. Moreover, the Fenchel cuts provided significant LP improvements in CF2 with short computational time. After the addition of such cuts, the LP of CF2 became $23.32 \%$ better than $\mathrm{CF} 1$, on average.

Table 16 - Comparison of linear relaxations between formulations CF1 and CF2.

\begin{tabular}{|c|c|c|c|c|c|c|c|c|}
\hline \multirow[b]{2}{*}{ ID } & \multicolumn{2}{|c|}{ CF1 } & \multicolumn{6}{|c|}{ CF2 } \\
\hline & Time (s) & $L P 1$ & Time (s) & $L P 2_{A}$ & $\operatorname{Gap}_{A}$ & Time (s) & $L P 2_{B}$ & $G a p_{B}$ \\
\hline 1 & 0.90 & 720 & 0.35 & 885 & 18.64 & 13.64 & 930 & 22.58 \\
\hline 2 & 0.85 & 720 & 0.32 & 885 & 18.64 & 10.37 & 930 & 22.58 \\
\hline 3 & 1.55 & 760 & 0.49 & 925 & 17.84 & 39.45 & 990 & 23.23 \\
\hline 4 & 1.67 & 770 & 0.50 & 925 & 16.76 & 31.01 & 970 & 20.62 \\
\hline 5 & 0.90 & 623.34 & 0.30 & 788.34 & 20.93 & 23.01 & 791.67 & 21.26 \\
\hline 6 & 1.70 & 830 & 0.51 & 1075 & 22.79 & 48.33 & 1080 & 23.15 \\
\hline 7 & 1.96 & 1275 & 0.55 & 1640 & 22.26 & 22.57 & 1680 & 24.11 \\
\hline 8 & 0.27 & 707.06 & 0.16 & 864.38 & 18.20 & 1.28 & 910.77 & 22.37 \\
\hline 9 & 0.66 & 853.42 & 0.27 & 1065.42 & 19.90 & 3.91 & 1100 & 22.42 \\
\hline 10 & 0.37 & 320 & 0.17 & 420 & 23.81 & 7.85 & 420 & 23.81 \\
\hline 11 & 1.32 & 1040 & 0.41 & 1377.50 & 24.50 & 14.48 & 1391.67 & 25.27 \\
\hline 12 & 1.12 & 1043.67 & 0.36 & 1401.12 & 25.51 & 7.09 & 1447 & 27.87 \\
\hline 13 & 0.03 & 310 & 0.04 & 392.5 & 21.02 & 0.12 & 410 & 24.39 \\
\hline 14 & 0.84 & 987.34 & 0.30 & 1193 & 17.24 & 7.98 & 1260 & 21.64 \\
\hline 15 & 0.13 & 250 & 0.10 & 340 & 26.47 & 0.99 & 375 & 33.33 \\
\hline 16 & 0.48 & 509 & 0.20 & 606.67 & 16.10 & 12.45 & 626.67 & 18.78 \\
\hline 17 & 0.42 & 470 & 0.19 & 525 & 10.48 & 10.62 & 540 & 12.96 \\
\hline 18 & 0.38 & 325 & 0.17 & 425 & 23.53 & 7.66 & 445 & 26.97 \\
\hline 19 & 0.33 & 270 & 0.14 & 350 & 22.86 & 2.97 & 360 & 25.00 \\
\hline 20 & 0.37 & 306.67 & 0.17 & 422.50 & 27.42 & 5.88 & 446.67 & 31.34 \\
\hline 21 & 1.77 & 1120 & 0.54 & 1405 & 20.28 & 30.27 & 1450 & 22.76 \\
\hline 22 & 0.61 & 754 & 0.23 & 1009.09 & 25.28 & 4.60 & 1029.29 & 26.75 \\
\hline 23 & 5.11 & 1929.53 & 1.21 & 2478.34 & 22.14 & 46.72 & 2500 & 22.82 \\
\hline 24 & 4.13 & 2062.53 & 0.98 & 2597.23 & 20.59 & 45.53 & 2680 & 23.04 \\
\hline 25 & 1.16 & 969 & 0.42 & 1295 & 25.17 & 34.10 & 1305 & 25.75 \\
\hline 26 & 0.58 & 620 & 0.25 & 740 & 16.22 & 6.71 & 740 & 16.22 \\
\hline 27 & 0.45 & 584 & 0.19 & 710 & 17.75 & 4.28 & 730 & 20.00 \\
\hline 28 & 1.71 & 1110 & 0.59 & 1440 & 22.92 & 66.74 & 1450 & 23.45 \\
\hline 29 & 1.58 & 1110 & 0.57 & 1440 & 22.92 & 57.28 & 1450 & 23.45 \\
\hline 30 & 1.65 & 1094 & 0.56 & 1430 & 23.50 & 32.52 & 1450 & 24.55 \\
\hline 31 & 1.44 & 1090 & 0.51 & 1410 & 22.70 & 18.24 & 1420 & 23.24 \\
\hline 32 & 1.50 & 1010 & 0.55 & 1280 & 21.09 & 34.39 & 1295 & 22.01 \\
\hline 33 & 1.32 & 1000 & 0.51 & 1275 & 21.57 & 35.88 & 1300 & 23.08 \\
\hline 34 & 1.19 & 1016 & 0.43 & 1271.67 & 20.11 & 33.65 & 1305 & 22.15 \\
\hline Avg.: & 1.19 & & 0.39 & & 21.09 & 21.25 & & 23.32 \\
\hline
\end{tabular}

\subsubsection{Comparing the integer programs of formulations CF1 and CF2}

This section compares the output of the integer programs of CF1 and CF2. Both programs were run with CPLEX's default settings during a time limit of three hours. Table 17 presents for each formulation, the execution time (Time), the best lower bound $(L B)$, the objective value of the best integer solution $(U B)$, and the optimality gap (Gap) on the tested instances. For formulation 
CF2, we present the versions without and with Fenchel cuts, CF2 and CF2-Cuts, respectively. In column (Time), TL means that the integer program reached the time limit of three hours without proving the optimality. The best LB and UB are shown in bold font. The row (Rank) presents the number of instances in which the formulations proved the optimality or reached the best LB or UB. The results show that formulation CF2 is, in general, better than CF1 in the quality of lower and upper bounds. Except that for the version without cuts, CF2 performed a bit worse than $\mathrm{CF} 1$ in the quality of integer solutions. The average optimality gap of CF2 is worse than CF1. However, $\mathrm{CF} 2$ proved more optimal solutions than $\mathrm{CF} 1$. Formulation $\mathrm{CF} 2$ proved 11 optimal solutions against 5 of CF1. With the addition of the Fenchel cuts, CF2 proved the optimality for 18 out of the 34 tested instances. 
Table 17 - Results of the integer programs of formulations CF1 and CF2 in a time limit of three hours.

\begin{tabular}{|c|c|c|c|c|c|c|c|c|c|c|c|c|}
\hline \multirow[b]{2}{*}{ ID } & \multicolumn{4}{|c|}{ CF1 } & \multicolumn{4}{|c|}{ CF2 } & \multicolumn{4}{|c|}{ CF2-Cuts } \\
\hline & Time (s) & LB & UB & Gap & Time (s) & LB & UB & Gap & Time (s) & LB & UB & Gap \\
\hline 1 & TL & 826.24 & 1290 & 35.95 & TL & 931.25 & 1805 & 48.41 & $\mathrm{TL}$ & 940 & 1040 & 9.62 \\
\hline 2 & TL & 814.61 & 1260 & 35.35 & TL & 932.70 & 1705 & 45.30 & $\mathrm{TL}$ & 941.43 & 950 & 0.90 \\
\hline 3 & TL & 839.17 & 1350 & 37.84 & TL & 972.15 & 9910 & 90.19 & $\mathrm{TL}$ & 1000 & 1040 & 3.85 \\
\hline 4 & TL & 842.50 & 1430 & 41.08 & TL & 962.70 & 1845 & 47.82 & $\mathrm{TL}$ & 990 & 1900 & 47.89 \\
\hline 5 & TL & 820 & 1020 & 19.61 & 7546.46 & 870 & 870 & 0.00 & 1054.40 & 870 & 870 & 0.00 \\
\hline 6 & TL & 880.36 & 1540 & 42.83 & TL & 1075 & 2545 & 57.76 & $\mathrm{TL}$ & 1080 & 1340 & 19.40 \\
\hline 7 & TL & 1647.82 & 2325 & 29.13 & TL & 1680 & 12840 & 86.92 & $\mathrm{TL}$ & 1690 & 3525 & 52.06 \\
\hline 8 & 2258.93 & 950 & 950 & 0.00 & 77.64 & 950 & 950 & 0.00 & 77.37 & 950 & 950 & 0.00 \\
\hline 9 & TL & 1062.59 & 1430 & 25.69 & TL & 1098.43 & 1100 & 0.14 & 83.30 & 1100 & 1100 & 0.00 \\
\hline 10 & 4466.99 & 420 & 420 & 0.00 & 80.60 & 420 & 420 & 0.00 & 91.28 & 420 & 420 & 0.00 \\
\hline 11 & $\mathrm{TL}$ & 1373.34 & 1885 & 27.14 & $\mathrm{TL}$ & 1397.15 & 1420 & 1.61 & 323.13 & 1400 & 1400 & 0.00 \\
\hline 12 & TL & 1422.16 & 2010 & 29.25 & 1216.46 & 1450 & 1450 & 0.00 & 422.49 & 1450 & 1450 & 0.00 \\
\hline 13 & 2.59 & 420 & 420 & 0.00 & 0.69 & 420 & 420 & 0.00 & 0.50 & 420 & 420 & 0.00 \\
\hline 14 & $\mathrm{TL}$ & 1227.25 & 1650 & 25.62 & $\mathrm{TL}$ & 1249.73 & 1975 & 36.72 & 529.50 & 1270 & 1270 & 0.00 \\
\hline 15 & 1566.40 & 395 & 395 & 0.00 & 15.20 & 395 & 395 & 0.00 & 9.39 & 395 & 395 & 0.00 \\
\hline 16 & $\mathrm{TL}$ & 579.17 & 750 & 22.78 & 401.87 & 640 & 640 & 0.00 & 517.54 & 640 & 640 & 0.00 \\
\hline 17 & $\mathrm{TL}$ & 531.67 & 660 & 19.44 & $\mathrm{TL}$ & 576 & 590 & 2.37 & 568.90 & 580 & 580 & 0.00 \\
\hline 18 & TL & 442.50 & 490 & 9.69 & TL & 452.23 & 460 & 1.69 & 120.26 & 460 & 460 & 0.00 \\
\hline 19 & TL & 350 & 360 & 2.78 & 21.63 & 360 & 360 & 0.00 & 17.79 & 360 & 360 & 0.00 \\
\hline 20 & $\mathrm{TL}$ & 417.34 & 520 & 19.74 & $\mathrm{TL}$ & 462.50 & 470 & 1.60 & 2076.64 & 470 & 470 & 0.00 \\
\hline 21 & TL & 1415 & 1990 & 28.89 & TL & 1440 & 11165 & 87.10 & 5801.61 & 1450 & 1450 & 0.00 \\
\hline 22 & TL & 1018.75 & 1245 & 18.17 & 483.84 & 1035 & 1035 & 0.00 & 295.88 & 1035 & 1035 & 0.00 \\
\hline 23 & $\mathrm{TL}$ & 2458.45 & 5715 & 56.98 & $\mathrm{TL}$ & 2482.78 & 22215 & 88.82 & $\mathrm{TL}$ & 2504.28 & 17790 & 85.92 \\
\hline 24 & $\mathrm{TL}$ & 2604.80 & 5115 & 49.08 & TL & 2669.98 & 20330 & 86.87 & $\mathrm{TL}$ & 2680 & 16825 & 84.07 \\
\hline 25 & TL & 1301.67 & 1685 & 22.75 & $\mathrm{TL}$ & 1310 & 2650 & 50.57 & $\mathrm{TL}$ & 1315 & 1320 & 0.38 \\
\hline 26 & $\mathrm{TL}$ & 724.29 & 940 & 22.95 & 6678.12 & 750 & 750 & 0.00 & 532.73 & 750 & 750 & 0.00 \\
\hline 27 & 6105.45 & 730 & 730 & 0.00 & 8269.66 & 730 & 730 & 0.00 & 204.87 & 730 & 730 & 0.00 \\
\hline 28 & $\mathrm{TL}$ & 1457 & 2250 & 35.24 & $\mathrm{TL}$ & 1547.15 & 3040 & 49.11 & $\mathrm{TL}$ & 1565 & 13420 & 88.34 \\
\hline 29 & $\mathrm{TL}$ & 1313.58 & 2160 & 39.19 & $\mathrm{TL}$ & 1520.56 & 4000 & 61.99 & $\mathrm{TL}$ & 1545 & 2040 & 24.26 \\
\hline 30 & $\mathrm{TL}$ & 1444.29 & 2165 & 33.29 & $\mathrm{TL}$ & 1557.50 & 3615 & 56.92 & $\mathrm{TL}$ & 1560.84 & 3500 & 55.40 \\
\hline 31 & $\mathrm{TL}$ & 1425.38 & 2070 & 31.14 & TL & 1552.50 & 2705 & 42.61 & $\mathrm{TL}$ & 1554.45 & 3480 & 55.33 \\
\hline 32 & $\mathrm{TL}$ & 1275.56 & 1830 & 30.30 & $\mathrm{TL}$ & 1356.67 & 3235 & 58.06 & $\mathrm{TL}$ & 1360 & 2185 & 37.76 \\
\hline 33 & $\mathrm{TL}$ & 1273.17 & 1920 & 33.69 & $\mathrm{TL}$ & 1386.67 & 2705 & 48.74 & $\mathrm{TL}$ & 1390 & 2755 & 49.55 \\
\hline 34 & TL & 1269.45 & 1920 & 33.88 & TL & 1336 & 3145 & 57.52 & $\mathrm{TL}$ & 1380 & 2205 & 37.41 \\
\hline Rank & & 5 & 16 & 5 & & 11 & 14 & 11 & & 34 & 24 & 18 \\
\hline Avg. & & & & 25.28 & & & & 32.61 & & & & 19.18 \\
\hline
\end{tabular}




\subsubsection{Comparing formulation CF2 with EF1, EF2 and EF3}

This section analyzes the lower bounds provided by the three extended formulations EF1, EF2 and EF3 compared to the lower bounds of the compact formulation CF2-Cuts. The column generation algorithms were run using CPLEX's default settings but with a single thread.

The results are shown in Table 18. The table presents for each formulation, the execution time (Time), the lower bound ( $L B)$ and the gap (Gap) to the best-known integer solution (Best $U B$ ) reported in Table 17. The lower bounds of CF2-Cuts were obtained with a time limit of three hours. The best gaps are shown in bold font, and shaded rows are instances for which the optimality was proven. The results demonstrate that, in general, the extended formulation EF2 provides the best lower bounds for problem HSTP-A. It generated the best lower bounds in 28 out of the 34 tested instances, against 23 ranked by the compact formulation CF2-Cuts. Also, the average gap of EF2 is smaller than the one of CF2-Cuts. The other two extended formulations, $\mathrm{EF} 1$ and EF3, do not compete with EF2 and CF2-Cuts in the quality of lower bounds, but they provide gaps that are close to the optimal solutions. Concerning computational time, formulation EF1 produced the best results among all formulations. On the other hand, the sequential version of the column generation algorithm of formulation EF3 is a very time-consuming process, and we had to solve the subproblems in parallel to achieve feasible computational time. 
Table 18 - The lower bounds of formulations EF1, EF2 and EF3 compared to lower bounds of formulation CF2-Cuts.

\begin{tabular}{|c|c|c|c|c|c|c|c|c|c|c|c|c|c|}
\hline \multirow[b]{2}{*}{ ID } & \multirow[b]{2}{*}{ Best UB } & \multicolumn{3}{|c|}{ CF2-Cuts (3 h) } & \multicolumn{3}{|c|}{ EF1 } & \multicolumn{3}{|c|}{ EF2 } & \multicolumn{3}{|c|}{ EF3 } \\
\hline & & Time (s) & LB & Gap & Time (s) & LB & Gap & Time (s) & LB & Gap & Time (s) & LB & Gap \\
\hline 1 & 1040 & $\mathrm{TL}$ & 940 & 9.62 & 24.41 & 930 & 10.58 & 47.16 & 950 & 8.65 & 191.84 & 923.34 & 11.22 \\
\hline 2 & 950 & $\mathrm{TL}$ & 941.43 & 0.90 & 24.79 & 930 & 2.11 & 47.89 & 950 & 0.00 & 181.99 & 923.34 & 2.81 \\
\hline 3 & 1040 & TL & 1000 & 3.85 & 41.01 & 990 & 4.81 & 62.40 & 1010 & 2.88 & 443.43 & 965 & 7.21 \\
\hline 4 & 1430 & $\mathrm{TL}$ & 990 & 30.77 & 38.00 & 970 & 32.17 & 64.63 & 990 & 30.77 & 819.46 & 955 & 33.22 \\
\hline 5 & 870 & 1054.40 & 870 & 0.00 & 25.72 & 791.67 & 9.00 & 35.82 & 870 & 0.00 & 156.03 & 865 & 0.57 \\
\hline 6 & 1340 & $\mathrm{TL}$ & 1080 & 19.40 & 41.30 & 1080 & 19.40 & 75.69 & 1080 & 19.40 & 442.19 & 1075 & 19.78 \\
\hline 7 & 2325 & $\mathrm{TL}$ & 1690 & 27.31 & 40.84 & 1680 & 27.74 & 118.60 & 1690 & 27.31 & 1168.52 & 1648.34 & 29.10 \\
\hline 8 & 950 & 77.37 & 950 & 0.00 & 6.22 & 910.77 & 4.13 & 22.19 & 930 & 2.11 & 36.01 & 892.45 & 6.06 \\
\hline 9 & 1100 & 83.30 & 1100 & 0.00 & 21.29 & 1100 & 0.00 & 92.47 & 1100 & 0.00 & 150.30 & 1070.63 & 2.67 \\
\hline 10 & 420 & 91.28 & 420 & 0.00 & 7.62 & 420 & 0.00 & 19.66 & 420 & 0.00 & 19.15 & 420 & 0.00 \\
\hline 11 & 1400 & 323.13 & 1400 & 0.00 & 46.17 & 1391.67 & 0.59 & 108.70 & 1400 & 0.00 & 407.97 & 1384.38 & 1.12 \\
\hline 12 & 1450 & 422.49 & 1450 & 0.00 & 26.08 & 1447 & 0.21 & 88.33 & 1450 & 0.00 & 329.46 & 1405.50 & 3.07 \\
\hline 13 & 420 & 0.50 & 420 & 0.00 & 1.81 & 410 & 2.38 & 3.38 & 417.15 & 0.68 & 1.68 & 400 & 4.76 \\
\hline 14 & 1270 & 529.50 & 1270 & 0.00 & 25.77 & 1260 & 0.79 & 71.02 & 1270 & 0.00 & 270.16 & 1208.75 & 4.82 \\
\hline 15 & 395 & 9.39 & 395 & 0.00 & 2.58 & 375 & 5.06 & 8.13 & 387 & 2.03 & 4.35 & 375 & 5.06 \\
\hline 16 & 640 & 517.54 & 640 & 0.00 & 12.89 & 626.67 & 2.08 & 27.70 & 640 & 0.00 & 43.98 & 620 & 3.13 \\
\hline 17 & 580 & 568.90 & 580 & 0.00 & 12.85 & 540 & 6.90 & 21.11 & 580 & 0.00 & 48.20 & 565 & 2.59 \\
\hline 18 & 460 & 120.26 & 460 & 0.00 & 8.85 & 445 & 3.26 & 19.87 & 455 & 1.09 & 27.32 & 442.50 & 3.80 \\
\hline 19 & 360 & 17.79 & 360 & 0.00 & 5.68 & 360 & 0.00 & 13.01 & 360 & 0.00 & 29.02 & 350 & 2.78 \\
\hline 20 & 470 & 2076.64 & 470 & 0.00 & 8.95 & 446.67 & 4.96 & 20.35 & 470 & 0.00 & 27.31 & 457.50 & 2.66 \\
\hline 21 & 1450 & 5801.61 & 1450 & 0.00 & 39.34 & 1450 & 0.00 & 97.66 & 1450 & 0.00 & 577.28 & 1410 & 2.76 \\
\hline 22 & 1035 & 295.88 & 1035 & 0.00 & 16.40 & 1029.29 & 0.55 & 51.53 & 1029.29 & 0.55 & 88.19 & 1019.50 & 1.50 \\
\hline 23 & 5715 & $\mathrm{TL}$ & 2504.28 & 56.18 & 158.18 & 2500 & 56.26 & 302.46 & 2505 & 56.17 & 10597.09 & 2478.34 & 56.63 \\
\hline 24 & 5115 & $\mathrm{TL}$ & 2680 & 47.61 & 128.38 & 2680 & 47.61 & 208.42 & 2680 & 47.61 & 11651.14 & 2614.45 & 48.89 \\
\hline 25 & 1320 & $\mathrm{TL}$ & 1315 & 0.38 & 32.70 & 1305 & 1.14 & 72.36 & 1313.34 & 0.50 & 369.13 & 1300 & 1.52 \\
\hline 26 & 750 & 532.73 & 750 & 0.00 & 14.47 & 740 & 1.33 & 49.56 & 750 & 0.00 & 66.27 & 750 & 0.00 \\
\hline 27 & 730 & 204.87 & 730 & 0.00 & 9.34 & 730 & 0.00 & 37.01 & 730 & 0.00 & 25.98 & 725 & 0.68 \\
\hline 28 & 2250 & TL & 1565 & 30.44 & 63.53 & 1450 & 35.56 & 119.56 & 1570 & 30.22 & 950.67 & 1540 & 31.56 \\
\hline 29 & 2040 & $\mathrm{TL}$ & 1545 & 24.26 & 59.86 & 1450 & 28.92 & 106.92 & 1570 & 23.04 & 923.66 & 1530.50 & 24.98 \\
\hline 30 & 2165 & $\mathrm{TL}$ & 1560.84 & 27.91 & 62.21 & 1450 & 33.03 & 126.22 & 1590 & 26.56 & 790.74 & 1560 & 27.94 \\
\hline 31 & 2070 & $\mathrm{TL}$ & 1554.45 & 24.91 & 57.02 & 1420 & 31.40 & 94.32 & 1570 & 24.15 & 545.36 & 1545 & 25.36 \\
\hline 32 & 1830 & $\mathrm{TL}$ & 1360 & 25.68 & 48.47 & 1295 & 29.23 & 72.45 & 1390 & 24.04 & 884.74 & 1359.67 & 25.70 \\
\hline 33 & 1920 & $\mathrm{TL}$ & 1390 & 27.60 & 54.01 & 1300 & 32.29 & 75.38 & 1410 & 26.56 & 655.71 & 1371.67 & 28.56 \\
\hline 34 & 1920 & $\mathrm{TL}$ & 1380 & 28.13 & 46.06 & 1305 & 32.03 & 61.65 & 1390 & 27.60 & 948.29 & 1340.28 & 30.19 \\
\hline Rank: & & & & 23 & & & 7 & & & 28 & & & 2 \\
\hline Avg.: & & & & 11.32 & & & 13.69 & & & 11.23 & & & 13.31 \\
\hline
\end{tabular}


Table 19 shows the number of generated columns $(\mathrm{NoC})$ for each extended formulation. The last row of the table shows the average time per column (Avg. T/C). These numbers provide some measure on how fast each subproblem is solved. They show that EF1 and EF2 provided the best execution time performance in the tested instances.

Table 19 - Number of generated columns (NoC) in formulations EF1, EF2 and EF3.

\begin{tabular}{|c|c|c|c|c|c|c|c|}
\hline \multirow[b]{2}{*}{ ID } & \multicolumn{3}{|c|}{ EF1 } & \multicolumn{2}{|c|}{ EF2 } & \multicolumn{2}{|c|}{ EF3 } \\
\hline & Time (s) & NoC $\lambda$ & NoC $\chi$ & Time (s) & NoC $\lambda$ & Time (s) & $\operatorname{NoC} \lambda$ \\
\hline 1 & 24.41 & 3447 & 728 & 47.16 & 1782 & 191.84 & 1145 \\
\hline 2 & 24.79 & 3610 & 754 & 47.89 & 1770 & 181.99 & 1120 \\
\hline 3 & 41.01 & 4490 & 1113 & 62.40 & 2032 & 443.43 & 1622 \\
\hline 4 & 38.00 & 4465 & 1068 & 64.63 & 2065 & 819.46 & 3133 \\
\hline 5 & 25.72 & 3182 & 996 & 35.82 & 1412 & 156.03 & 1353 \\
\hline 6 & 41.30 & 4650 & 968 & 75.69 & 2183 & 442.19 & 1849 \\
\hline 7 & 40.84 & 5123 & 866 & 118.60 & 3517 & 1168.52 & 2105 \\
\hline 8 & 6.22 & 1653 & 354 & 22.19 & 1266 & 36.01 & 676 \\
\hline 9 & 21.29 & 3428 & 600 & 92.47 & 2703 & 150.30 & 1075 \\
\hline 10 & 7.62 & 1883 & 540 & 19.66 & 891 & 19.15 & 413 \\
\hline 11 & 46.17 & 4802 & 696 & 108.70 & 3191 & 407.97 & 1495 \\
\hline 12 & 26.08 & 4128 & 506 & 88.33 & 2971 & 329.46 & 1301 \\
\hline 13 & 1.81 & 496 & 103 & 3.38 & 347 & 1.68 & 157 \\
\hline 14 & 25.77 & 3727 & 603 & 71.02 & 2767 & 270.16 & 1161 \\
\hline 15 & 2.58 & 834 & 256 & 8.13 & 589 & 4.35 & 269 \\
\hline 16 & 12.89 & 2410 & 620 & 27.70 & 1195 & 43.98 & 669 \\
\hline 17 & 12.85 & 2155 & 687 & 21.11 & 999 & 48.20 & 709 \\
\hline 18 & 8.85 & 2006 & 616 & 19.87 & 869 & 27.32 & 571 \\
\hline 19 & 5.68 & 1423 & 511 & 13.01 & 673 & 29.02 & 744 \\
\hline 20 & 8.95 & 2067 & 641 & 20.35 & 933 & 27.31 & 499 \\
\hline 21 & 39.34 & 4902 & 588 & 97.66 & 2933 & 577.28 & 1337 \\
\hline 22 & 16.40 & 2883 & 486 & 51.53 & 1915 & 88.19 & 738 \\
\hline 23 & 158.18 & 9879 & 1530 & 302.46 & 6096 & 10597.09 & 3288 \\
\hline 24 & 128.38 & 8878 & 1653 & 208.42 & 5741 & 11651.14 & 4411 \\
\hline 25 & 32.70 & 4375 & 688 & 72.36 & 2546 & 369.13 & 1465 \\
\hline 26 & 14.47 & 2693 & 561 & 49.56 & 1686 & 66.27 & 726 \\
\hline 27 & 9.34 & 2174 & 423 & 37.01 & 1409 & 25.98 & 476 \\
\hline 28 & 63.53 & 5074 & 1500 & 119.56 & 3282 & 950.67 & 1920 \\
\hline 29 & 59.86 & 5083 & 1467 & 106.92 & 3128 & 923.66 & 1979 \\
\hline 30 & 62.21 & 5091 & 1498 & 126.22 & 3277 & 790.74 & 1844 \\
\hline 31 & 57.02 & 5007 & 1430 & 94.32 & 3075 & 545.36 & 1774 \\
\hline 32 & 48.47 & 4511 & 1408 & 72.45 & 2601 & 884.74 & 2082 \\
\hline 33 & 54.01 & 4562 & 1424 & 75.38 & 2696 & 655.71 & 1790 \\
\hline 34 & 46.06 & 4406 & 1332 & 61.65 & 2472 & 948.29 & 2062 \\
\hline \multicolumn{2}{|c|}{ Avg. T/C: } & & 0.006 & & 0.028 & & 0.378 \\
\hline
\end{tabular}

\subsubsection{Results for problem HSTP-B}

This section reports the results of formulations CF2-Cuts and EF2 adapted to problem HSTP-B. For this problem, we consider the instances proposed by Souza (2000). The instances are shown in Table 20. As mentioned in Section 2.4, these instances have optimal solutions known, which is a contribution of previous studies using heuristic solutions (SOUZA; MACULAN; OCHI, 2004; SANTOS; OCHI; SOUZA, 2005; SAVINIEC et al., 2013; DORNELES; ARAÚJO; BURIOL, 2014) and column generation lower bounds (SANTOS et al., 2012; DORNELES; 
ARAÚJO; BURIOL, 2017). According to Santos, Ochi and Souza (2005), the objective function weighting parameters for these instances are: $\alpha_{7}=1, \alpha_{8}=3$ and $\alpha_{9}=9$.

Table 20 - Features of the instances proposed by Souza (2000).

\begin{tabular}{rrrrrrrr}
\hline ID & $|C|$ & $|T|$ & $|D|$ & $|H|$ & $\sum_{r \in R} \widetilde{\theta}_{r}$ & $\sum_{r \in R} \widetilde{\pi}_{r}$ & Optimal value \\
\hline 1 & 3 & 8 & 5 & 5 & 75 & 21 & 202 \\
2 & 6 & 14 & 5 & 5 & 150 & 29 & 333 \\
3 & 8 & 16 & 5 & 5 & 200 & 4 & 423 \\
4 & 12 & 23 & 5 & 5 & 300 & 41 & 652 \\
5 & 13 & 31 & 5 & 5 & 325 & 71 & 762 \\
6 & 14 & 30 & 5 & 5 & 350 & 63 & 756 \\
7 & 20 & 33 & 5 & 5 & 500 & 84 & 1017 \\
\hline
\end{tabular}

Table 21 - The lower bounds generated by formulation EF2 on the instances of Souza (2000).

\begin{tabular}{rrrrr}
\hline ID & Time (s) & NoC $\lambda$ & LB & Gap \\
\hline 1 & 14.66 & 371 & 202 & 0.00 \\
2 & 24.60 & 848 & 333 & 0.00 \\
3 & 22.54 & 904 & 423 & 0.00 \\
4 & 35.08 & 1364 & 652 & 0.00 \\
5 & 39.73 & 1677 & 762 & 0.00 \\
6 & 89.80 & 2025 & 756 & 0.00 \\
7 & 82.39 & 2216 & 1017 & 0.00 \\
\hline
\end{tabular}

Table 21 presents the results of EF2 to the instances of Souza (2000). The CPLEX was run with a single thread, and the remaining parameters were set to default. The column (Gap) presents the gap between the lower bounds $(L B)$ of EF2 and the optimal solutions. The results show that EF2 reached the optimal lower bounds for all instances. These results are competitive with Santos et al. (2012) and Dorneles, Araújo and Buriol (2017), which reported the same results with their column generation approaches.

Dorneles, Araújo and Buriol (2017) also reported results of a compact formulation based on a multi-commodity flow problem (CF-MCFP). In table 22, we compare these results with results of our compact formulation $\mathrm{CF} 2$-Cuts. The best results are shown in bold font. The program of CF2-Cuts was run with the same parameter settings used by Dorneles, Araújo and Buriol (2017), that are: CPLEX single thread and a time limit of two hours. The columns $\left(L P_{A}\right)$ and $(L P)$ show that the two formulations generated the same linear relaxation values. Regarding the integer programs, our formulation provided better lower bounds than CF-MCFP after two hours. On the other hand, the program of CF-MCFP found better integer solutions than the program of CF2-Cuts. While CF-MCFP ranked six best solutions, CF2-Cuts ranked only four best solutions. The CF2-Cuts also failed in finding a feasible solution to instance 7 during two hours. However, the lower bound found to instance 7 is optimal. We also observed that for instances 4 and 7, the Fenchel cuts led to optimal lower bounds (column $L P_{B}$ ). 
Table 22 - Formulation CF2-Cuts compared to formulation CF-MCFP on problem HSTP-B.

\begin{tabular}{|c|c|c|c|c|c|c|c|c|c|c|c|c|c|c|}
\hline \multirow[b]{2}{*}{ ID } & \multicolumn{8}{|c|}{ CF2-Cuts } & \multicolumn{6}{|c|}{ CF-MCFP } \\
\hline & Time (s) & $L P_{A}$ & Time (s) & $L P_{B}$ & Time (s) & LB & UB & Gap & Time (s) & LP & Time (s) & $\mathrm{LB}^{*}$ & UB & Gap \\
\hline 1 & 0.08 & 189 & 1.66 & 190 & 126.96 & 202 & 202 & 0.00 & 0.10 & 189 & TL & 190.24 & 202 & 5.82 \\
\hline 2 & 0.67 & 333 & 18.84 & 333 & 1482.61 & 333 & 333 & 0.00 & 0.40 & 333 & 196 & 333 & 333 & 0.00 \\
\hline 3 & 0.39 & 414 & 19.29 & 414 & $\mathrm{TL}$ & 418 & 444 & 5.86 & 0.20 & 414 & $\mathrm{TL}$ & 413.99 & 429 & 3.50 \\
\hline 4 & 2.08 & 643 & 83.92 & 652 & 1057.08 & 652 & 652 & 0.00 & 0.80 & 643 & 988 & 652 & 652 & 0.00 \\
\hline 5 & 4.05 & 756 & 273.95 & 757 & TL & 757.50 & 763 & 0.72 & 2.30 & 756 & TL & 756.02 & 777 & 2.70 \\
\hline 6 & 6.06 & 738 & 375.48 & 750 & TL & 750 & 935 & 19.79 & 2.80 & 738 & TL & 739.52 & 804 & 8.02 \\
\hline 7 & 12.69 & 999 & 842.92 & 1017 & TL & 1017 & - & - & 7.80 & 999 & TL & 1006.58 & 1645 & 38.81 \\
\hline Rank: & & & & & & 7 & 4 & & & & & 2 & 6 & \\
\hline
\end{tabular}

Dorneles, Araújo and Buriol (2017) did not report the lower bounds $(L B)$ of their integer programs after two hours. We calculated these values by the formula $L B=U B(1-G a p \div 100)$, where Gap was reported to be the CPLEX's optimality gap. 


\subsubsection{Results for problem HSTP-C}

This section reports the results of formulations CF2-Cuts and EF2 adapted to problem HSTP-C. For this problem, we consider the instances of Table 15. The objective function weighting parameters were set to: $\alpha_{7}=1, \alpha_{8}=3$ and $\alpha_{9}=9$.

The results are shown in Table 23. For this problem, instances 15 and 22 were proven to be infeasible. For the remaining instances, the table compares the lower bounds of formulations CF2Cuts and EF2. The columns (Gap) show the gap between the lower bound of each formulation and the best integer solution $(U B)$ of CF2-Cuts. The best gaps are shown in bold font, and shaded rows are instances for which the optimality was proven. For this problem, the compact formulation CF2-Cuts performed slightly better than EF2 in the quality of lower bounds. Both formulations ranked the same number of best lower bounds, and we could prove the optimality for 25 out of the 32 instances that are not infeasible. 
Table 23 - Results of formulations CF2-Cuts (TL $=3 \mathrm{~h}$ ) and EF2 on problem HSTP-C.

\begin{tabular}{|c|c|c|c|c|c|c|c|c|c|c|c|c|}
\hline \multirow[b]{2}{*}{ ID } & \multicolumn{8}{|c|}{ CF2-Cuts } & \multicolumn{4}{|c|}{ EF2 } \\
\hline & Time (s) & $L P_{A}$ & Time (s) & $L P_{B}$ & Time (s) & LB & UB & Gap & Time (s) & $\operatorname{NoC} \lambda$ & LB & Gap \\
\hline 1 & 0.37 & 682.50 & 7.50 & 689 & 1446.54 & 699 & 699 & 0.00 & 49.06 & 1828 & 699 & 0.00 \\
\hline 2 & 0.35 & 682.50 & 11.75 & 689 & 1689.78 & 699 & 699 & 0.00 & 49.83 & 1817 & 699 & 0.00 \\
\hline 3 & 0.54 & 727.50 & 11.70 & 735 & 3900.23 & 742 & 742 & 0.00 & 58.25 & 2114 & 742 & 0.00 \\
\hline 4 & 0.51 & 726.50 & 22.04 & 731 & 3977.16 & 734 & 734 & 0.00 & 59.99 & 2131 & 734 & 0.00 \\
\hline 5 & 0.35 & 627 & 39.46 & 627 & 729.99 & 631 & 631 & 0.00 & 32.32 & 1427 & 631 & 0.00 \\
\hline 6 & 0.56 & 771.50 & 24.53 & 772 & 2942.94 & 772 & 772 & 0.00 & 71.08 & 2282 & 772 & 0.00 \\
\hline 7 & 0.58 & 1197.50 & 14.15 & 1209 & 10799.32 & 1212 & 1212 & 0.00 & 95.15 & 3281 & 1212 & 0.00 \\
\hline 8 & 0.17 & 637.86 & 1.09 & 664.80 & 66.33 & 675 & 675 & 0.00 & 21.86 & 1293 & 669 & 0.89 \\
\hline 9 & 0.35 & 797.18 & 6.84 & 805.25 & 247.16 & 807 & 807 & 0.00 & 59.83 & 2200 & 806.25 & 0.09 \\
\hline 10 & 0.18 & 298 & 3.70 & 298 & 72.79 & 298 & 298 & 0.00 & 18.17 & 884 & 298 & 0.00 \\
\hline 11 & 0.45 & 942.19 & 9.01 & 948.50 & 1972.01 & 961 & 961 & 0.00 & 76.99 & 2908 & 959.87 & 0.12 \\
\hline 12 & 0.37 & 996.13 & 5.01 & 1014.74 & 498.25 & 1018 & 1018 & 0.00 & 82.87 & 2946 & 1014.74 & 0.32 \\
\hline 13 & 0.04 & 261.50 & 0.05 & 265 & 0.72 & 273 & 273 & 0.00 & 2.51 & 302 & 270.67 & 0.85 \\
\hline 14 & 0.36 & 911.23 & 8.33 & 930 & 761.33 & 933 & 933 & 0.00 & 48.35 & 2476 & 933 & 0.00 \\
\hline 15 & & & & & & Infeasib & & & & & & \\
\hline 16 & 0.24 & 472.67 & 5.39 & 477 & 485.21 & 481 & 481 & 0.00 & 22.55 & 1196 & 481 & 0.00 \\
\hline 17 & 0.22 & 455.50 & 10.20 & 457 & 308.88 & 457 & 457 & 0.00 & 20.56 & 1078 & 457 & 0.00 \\
\hline 18 & 0.18 & 309.25 & 3.81 & 316 & 100.77 & 319 & 319 & 0.00 & 19.62 & 859 & 319 & 0.00 \\
\hline 19 & 0.15 & 251 & 3.09 & 254 & 14.75 & 254 & 254 & 0.00 & 15.60 & 738 & 254 & 0.00 \\
\hline 20 & 0.19 & 310.50 & 3.79 & 317 & 224.26 & 325 & 325 & 0.00 & 24.83 & 1034 & 325 & 0.00 \\
\hline 21 & 0.62 & 1036 & 13.41 & 1050 & 6163.85 & 1050 & 1050 & 0.00 & 73.70 & 2760 & 1050 & 0.00 \\
\hline 22 & & & & & & Infeasib & & & & & & \\
\hline 23 & 1.51 & 1789.19 & 39.77 & 1812 & TL & 1813 & 2357 & 23.08 & 273.51 & 6107 & 1813 & 23.08 \\
\hline 24 & 1.03 & 1932.79 & 30.19 & 1983.50 & $\mathrm{TL}$ & 1988 & 2535 & 21.58 & 201.99 & 5832 & 1988 & 21.58 \\
\hline 25 & 0.47 & 904 & 27.97 & 909 & 343.97 & 912 & 912 & 0.00 & 56.54 & 2408 & 912 & 0.00 \\
\hline 26 & 0.29 & 570 & 8.94 & 570 & 245.98 & 570 & 570 & 0.00 & 37.02 & 1594 & 570 & 0.00 \\
\hline 27 & 0.21 & 549.75 & 4.55 & 552 & 140.12 & 552 & 552 & 0.00 & 27.67 & 1322 & 552 & 0.00 \\
\hline 28 & 0.63 & 1104 & 64.61 & 1105 & $\mathrm{TL}$ & 1122.13 & 1190 & 5.70 & 95.61 & 3067 & 1124 & 5.55 \\
\hline 29 & 0.68 & 1095 & 43.33 & 1096 & TL & 1112.94 & 1306 & 14.78 & 115.67 & 3136 & 1116 & 14.55 \\
\hline 30 & 0.61 & 1113 & 86.22 & 1114 & $\mathrm{TL}$ & 1131.40 & 1703 & 33.56 & 107.54 & 3154 & 1133 & 33.47 \\
\hline 31 & 0.56 & 1112 & 60.81 & 1113 & 2618.76 & 1127 & 1127 & 0.00 & 79.59 & 2966 & 1127 & 0.00 \\
\hline 32 & 0.57 & 999 & 53.11 & 999 & $\mathrm{TL}$ & 1010 & 1740 & 41.95 & 78.22 & 2658 & 1014 & 41.72 \\
\hline 33 & 0.54 & 999.50 & 51.71 & 1001 & $\mathrm{TL}$ & 1016 & 1081 & 6.01 & 67.91 & 2613 & 1018 & 5.83 \\
\hline 34 & 0.53 & 980 & 37.11 & 981 & 9355.78 & 1005 & 1005 & 0.00 & 73.85 & 2557 & 1005 & 0.00 \\
\hline Rank: & & & & & & & & 27 & & & & 27 \\
\hline Avg.: & & & & & & & & 4.58 & & & & 4.63 \\
\hline
\end{tabular}




\subsection{Final remarks}

In this chapter, we conducted a study with mixed-integer programming formulations for high school timetabling problems. We proposed two compact formulations CF1 and CF2, and three extended formulations EF1, EF2, and EF3. The first formulation is based on traditional models. The other four formulations are alternative models based on the concept of "patterns", for which variables represent all possible layouts of assigning requirements or teachers to periods within days or during the week. The alternative compact formulation CF2 allows the generation of new cuts, based on the Fenchel cuts technique, to strengthen its linear relaxation. Our three extended formulations EF1, EF2, and EF3 are based on this new model. As the extended formulations have huge numbers of variables that cannot be explicitly generated, we resort to column generation to solve their linear relaxations and provide lower bounds to the problem. We tested these formulations in three different HSTP problems. Regarding the compact formulations, we observed that:

- Formulation $\mathrm{CF} 2$ has a better linear relaxation than $\mathrm{CF} 1$. However, for large size instances, $\mathrm{CF} 1$ obtain better quality integer solutions than CF2.

- The Fenchel enumeration cutting planes technique provides cheap and strong cuts to CF2 on the considered problems. In some instances, the addition of such cuts was sufficient to reach the optimal lower bounds.

- While the classical formulation CF1 proved optimality only for small size instances, the alternative compact formulation $\mathrm{CF} 2$, with the addition of the Fenchel cuts, proved the optimality for small and also for medium size instances.

- In comparison with the state-of-the-art compact formulation (DORNELES; ARAÚJO; BURIOL, 2017) to problem HSTP-B, our compact formulation CF2 (with cuts) performs better in the quality of lower bounds, but it is a bit worse in the quality of integer solutions.

- Formulation CF2 (with cuts) performs better for problems that have a more restricted feasible region, as in problem HSTP-C.

Regarding the proposed extended formulations, we noted that:

- The best formulation EF2 provides a cheap way of obtaining strong lower bounds to the three tested problems. In most of the cases, it provided optimal lower bounds.

- The best formulation EF2 is competitive in the quality of lower bounds with previous extended formulations proposed in the literature (SANTOS et al., 2012; DORNELES; ARAÚJO; BURIOL, 2017). 
Lastly, the alternative formulations helped to prove optimality for most of the tested instances for which the traditional formulation CF1 failed.

Future extensions of this research can be done in order to devise a branch-and-price method that exploits the column generations proposed in this chapter. 
CHAPTER

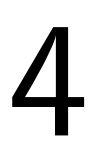

4

4

\section{PARALLEL METAHEURISTICS}

This chapter is strongly based on the paper "Parallel local search algorithms for high school timetabling problems" (SAVINIEC; SANTOS; COSTA, 2018) which is part of this thesis.

Due to the difficulty of devising MIP formulations for high school timetabling problems that can be efficiently solved by MIP solvers, most of the literature has been concerned with metaheuristic techniques (PILLAY, 2014). The primary goal of such studies is to develop strategies to generate good quality solutions within reasonable computational effort. Recently proposed metaheuristics for the HSTP can be found in Fonseca and Santos (2014), Dorneles, Araújo and Buriol (2014), Fonseca et al. (2016), Fonseca, Santos and Carrano (2016), Saviniec and Constantino (2017) and Skoullis, Tassopoulos and Beligiannis (2017). These methods are shown to be efficient in the sense that near-optimal solutions are consistently found for different input instances.

In this chapter, we investigate parallel metaheuristics for the HSTP, in which different solution methods (agents) are run concurrently in different processor threads. The motivation is two-fold. On the one hand, the availability of multi-processor machines and appropriate coding schemes make the use of parallel algorithms more accessible than ever. On the other hand, although the literature is abundant in parallel solution methods for similar complex problems (SUBRAMANIAN et al., 2010; BOŻEJKO; PEMPERA; SMUTNICKI, 2013; SÁNCHEZ-ORO et al., 2015; LUQUE; ALBA, 2015), very little attention has been given to the design of parallel methods for the HSTP. The reader is referred to Section 4.1 for a closer look at these contributions (ABRAMSON, 1991; ABRAMSON; ABELA, 1992; SRNDIC et al., 2009).

This chapter provides an exploratory study that investigates some questions related to the design of parallel metaheuristics in the context of the HSTP. Our central research questions can be summarized as: (1) What are good parallel strategies for the HSTP? (2) Can these strategies improve the performance of stand-alone metaheuristics? (3) Are the proposed parallel metaheuristics competitive with state-of-the-art methods? 
To provide some insight into these questions, we carry out a thorough computational study that tests all parallelization schemes resulting from combinations of the following characteristics:

Agents cooperation: we wish to test the effect of allowing the agents to cooperate, by sharing good solutions among threads.

Diversification: we wish to examine the effect of using all agents as search intensification mechanisms or allowing at least one agent to diversify the search.

Agents diversity: we wish to test homogeneous and heterogeneous algorithms. An algorithm is homogeneous when all threads execute the same metaheuristic, and heterogeneous otherwise.

As stand-alone metaheuristics, we use the iterated local search from Saviniec et al. (2013) and propose versions of tabu search, simulated annealing, and late acceptance strategy. We compare the performance of the different parallel algorithm variants against each other, against the stand-alone metaheuristics and two state-of-the-art algorithms for problems HSTP-B and HSTP-C (DORNELES; ARAÚJO; BURIOL, 2014; FONSECA et al., 2016).

The chapter is organized as follows. Section 4.1 gives an introduction to different parallelization schemes and reviews the existing parallel algorithms for the HSTP. Section 4.2 explains our parallel metaheuristics. Section 4.3 presents our computational experiments and Section 4.4 concludes the chapter with final remarks and suggestions for further investigations. Appendix A completes this chapter with details on the stand-alone metaheuristics and their parameter setting procedures.

\subsection{Existing parallel algorithms for high school timetabling}

Metaheuristics can be classified into population-based $(P B)$ and trajectory-based $(T B)$ methods (ALBA; LUQUE; NESMACHNOW, 2013). Population-based metaheuristics are characterized by keeping a pool of solutions (e.g., genetic algorithms, ant colony optimization and particle swarm optimization). The method starts with an initial population and employs, at each step, stochastic operators to evolve toward better quality populations. According to Alba, Luque and Nesmachnow (2013), two classical parallel algorithm frameworks have been used for population-based metaheuristics:

- Parallel individuals evaluation (PB-PIE): each individual in the population can be evaluated in parallel.

- Parallel islands (PB-PI): the initial population is split into a set of subpopulations (islands) in which a sequential algorithm can be employed. In this framework, the islands often exchange individuals among each other to diversify the subpopulations and prevent early convergence. 
Trajectory-based metaheuristics are characterized by methods which employ a single current solution. The method starts with an initial solution and at each step, goes through neighborhoods of the current solution tracing trajectories in the search space of the problem. Some well-known metaheuristics in this class are: simulated annealing, tabu search, iterated local search and variable neighborhood search. Three classical parallel algorithm frameworks have been used in the literature for trajectory-based metaheuristics according to Alba, Luque and Nesmachnow (2013):

- Parallel moves (TB-PM): the neighborhood of the current solution is explored in parallel. This framework is usually implemented by a manager/workers technique. At each step, the manager replicates the current solution to a set of parallel workers, each worker then computes the objective function of one of the neighboring solutions and returns it to the manager, who takes decisions.

- Move acceleration (TB-MA): the objective function of a single solution is evaluated in parallel. This framework is an attractive approach when the sequential evaluation of the objective function is both time-consuming and can be decomposed into small parts to be computed in parallel.

- Parallel multi-start (TB-PMS): several asynchronous threads of trajectory-based methods run simultaneously to compute high-quality solutions. These threads may be homogeneous (same method) or heterogeneous (distinct methods). They may or may not cooperate with each other. They may also start with the same solution seed or from different ones.

Luque and Alba (2015) state that parallel strategies for trajectory-based methods have been less studied than for population-based ones. For HSTP, we have found only three studies related to parallel metaheuristics. Abramson (1991) proposed a simulated annealing which employs a series of locks to enable several processes to perform simultaneous moves in the same timetable data structure (TB-PM). Abramson and Abela (1992) proposed a genetic algorithm that, at each step, generates and evaluates individuals in parallel (PB-PIE). Srndic et al. (2009) proposed a genetic algorithm approach in which the global population is divided into small subpopulations that are managed by different processes (PB-PI).

In this chapter, we focus on trajectory-based methods. Particularly, we propose parallel multi-start methods for the HSTP using as agents sequential iterated local search (LOURENÇO; MARTIN; STÜTZLE, 2003), tabu search (GLOVER, 1990), simulated annealing (KIRKPATRICK; GELATT; VECCHI, 1983) and late acceptance strategy (BURKE; BYKOV, 2008) metaheuristics. Details of these metaheuristics are presented in Appendix A. In the next section, we concentrate on the parallel algorithms. 


\subsection{Proposed parallel metaheuristics}

The proposed algorithms follow the trajectory-based parallel multi-start (TB-PMS) scheme described in Section 4.1 and employ manager/workers strategies with shared memories. We propose two approaches. The first is based on central memory (CRAINIC et al., 2004) and the second is based on diversification and intensification memories (JIN; CRAINIC; LØKKETANGEN, 2014).

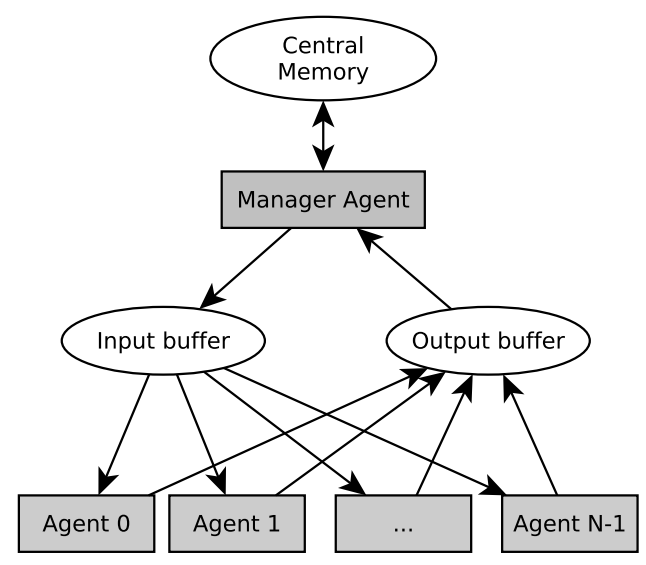

(a) Central memory based: a parallel multi-start framework using a manager/workers strategy and a central memory of solutions.

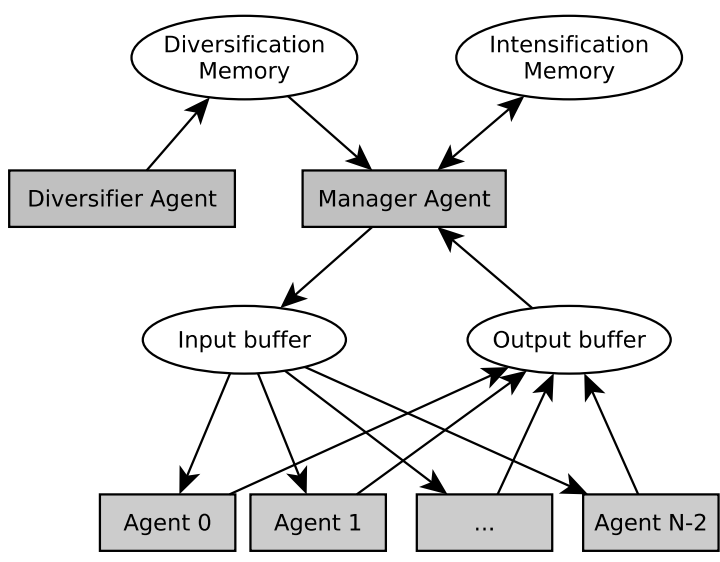

(b) Diversification-intensification memory based: a parallel multi-start framework with diversification and intensification memories.

Figure 5 - Parallel multi-start metaheuristic frameworks.

\subsubsection{Central memory based algorithms}

The central memory based (CMB) framework, shown in Figure 5a, has a central memory which keeps up to $X$ solutions, a manager procedure, $N$ metaheuristic worker agents and two synchronization buffers, input and output. The main idea in this framework is that a group of metaheuristic agents can execute concurrently while possibly cooperating with each other through an exchange of current solutions. This exchange is done via the central memory, which keeps a set of elite solutions.

Each agent pulls a solution from the input buffer, operates on it for a parameter-defined amount of time, and then returns its best solution to the output buffer. The manager is responsible for implementing the policies that will determine the exchange of solutions between the two intermediate buffers (input and output) and the central memory. In our implementations with this framework, the following policies are adopted:

- Memory initialization: the central memory is initialized with solutions generated by the constructive heuristic described in Algorithm 7, see Appendix A. 
- Input selection: a solution is randomly selected from the central memory and put into the input buffer whenever requested by an agent.

- Output acceptance: when the manager retrieves a solution $Z_{r}$ from the output buffer, the solution $Z_{r}$ is compared sequentially with solutions in the central memory. A firstimprovement strategy is adopted, i.e., the first visited solution that is worse than or equal to $Z_{r}$ is replaced. If no solution meets this criterion, then $Z_{r}$ is discarded.

- Agents' execution time: each of the $N$ agents is allowed to run for Y seconds before pushing its best solution into the output buffer and requesting a new solution from the input buffer. Short execution time defines cooperative agents, while long execution time defines independent agents.

\subsubsection{Diversification-intensification memory based algorithms}

The diversification-intensification memory based (DIMB) framework, shown in Figure $5 b$, is an extension of the CMB framework. The central memory is now divided into a 'diversification memory' and an 'intensification memory'. The first memory keeps a set of non-elite solutions to diversify the search while the second maintains a set of elite solutions.

One agent is responsible for providing diversified solutions to the diversification memory while all others search for better quality solutions. The details of our implementation are as follows:

- Memory initialization: the two memories are initialized with solutions generated by the constructive heuristic described in Algorithm 7.

- Input selection: whenever requested by an agent, a solution is selected from the diversification memory with probability $\rho$ and from the intensification memory with probability $(1-\rho)$.

- Output acceptance: when the manager retrieves a solution $Z_{r}$ from the output buffer, it accepts the solution into the intensification memory if it is better than or equal to a solution randomly chosen in the intensification memory. Otherwise, $Z_{r}$ is discarded.

- Agents' execution time: each of the $N-1$ intensification agents is allowed to run for Y seconds before pushing its best solution into the output buffer and requesting a new solution from the input buffer.

- Diversification memory update: The solutions of this memory are continuously replaced by solutions with better or equivalent quality generated by the diversifier agent during run-time. The diversifier is, usually, a metaheuristic with slow convergence or that quickly converges to poor local optima and keeps performing plain moves (moving to other 
solutions with the same quality). In our implementation, the diversifier agent is a late acceptance strategy metaheuristic with plain moves and set with parameters that lead to premature convergence. Whenever the late acceptance strategy updates its current solution $Z_{c}$, the solution $Z_{c}$ is compared with a random solution $Z_{d}$ in the diversification memory. If $Z_{c}$ is better or equal to $Z_{d}$ in terms of objective value, the metaheuristic replaces $Z_{d}$ by $Z_{c}$.

In the next section, we test these two frameworks with several parameter configurations. We investigate cases in which the agents are homogeneous or heterogeneous, and cooperative or independent.

\subsection{Computational experiments}

In this section, we conduct a thorough computational study to evaluate the performance of the proposed parallel methods. All algorithms were coded in $\mathrm{C}++$ and compiled with GNU Compiler Collection 4.4.7. The experiments were done on a server running Red Hat Enterprise Linux 6.5. The hardware is composed of two CPU Intel Xeon E5-2680v2 (2.8 GHz) and 128 GB of RAM. To implement parallelism, we employed the POSIX Threads Library (Pthreads) available in the GNU Compiler Collection. In all experiments reported, the metaheuristics are allowed to execute during a time limit of 625 seconds, and 25 trials (different seeds) are used for each instance. The seeds were generated by standard $\mathrm{C}++$ libraries, and we used the same seeds for every metaheuristic configuration.

The experiments have been set up in two phases: in Section 4.3.1, we compare the different proposed methods against each other on problem HSTP-A. The following tests are executed:

Section 4.3.1.1: tests with the CMB parallel framework proposed in Section 4.2.1.

Section 4.3.1.2: tests with the DIMB parallel framework proposed in Section 4.2.2.

Section 4.3.1.3: tests comparing the best sequential and parallel algorithm configurations.

Section 4.3.1.4: tests comparing our best parallel algorithm with the MIP program of formulation CF2 proposed in Chapter 3.

Then, in Section 4.3.2, the best parallel algorithm is also adapted to problems HSTP-B and HSTP-C and compared with state-of-the-art literature methods:

Section 4.3.2.1: tests comparing our best parallel algorithm with methods reported to problem HSTP-B. 
Section 4.3.2.2: tests comparing our best parallel algorithm with the solver winner of the ITC-2011 on problem HSTP-C.

The interested reader is referred to Appendix A, where the used stand-alone metaheuristics are described along with the procedure we used for setting their best parameters.

\subsubsection{Comparisons among proposed methods}

For tests 4.3.1.1 to 4.3.1.2, we use the reduced set of six instances $(6,7,10,13,24$, and 26) from Table 15, which were used to calibrate the sequential metaheuristics in Section A.6. For tests 4.3.1.3 to 4.3.1.4, we use all 34 instances described in Table 15.

\subsubsection{Results for central memory based algorithms}

The two parallel frameworks CMB and DIMB, outlined in Section 4.2, generate different algorithms for different combinations of metaheuristic agents. Also, agents with short execution time (parameter $Y$ ) produce cooperative searches while agents with long execution time lead to independent searches. In this section, we analyze algorithms which include homogeneous, heterogeneous, cooperative and independent agents. We assess how much these parallel algorithms are better than the sequential ones and how cooperation can play a role in the quality of solutions.

We first analyze four cases of CMB parallel metaheuristics. In each case, the agents are homogeneous threads of one of the metaheuristics ILS, TS, SA or LAS described in Section A.5. For each case, we tested configurations in which the central memory contains $X=(1,4$, $8,16,32)$ solutions and agents time $Y=(1,2,5,10,30,90,315,625)$ seconds. Cases with homogeneous (HM) ILS showed the overall best results.

Figure 6 plots the best configurations with homogeneous ILS. In this figure and the remaining figures in this chapter, the $y$-axis represents normalized objective values obtained as follows. Let $Z_{j}$ be a timetable solution for the $j^{\text {th }}$ instance, its normalized objective value is given by $\operatorname{NOV}\left(Z_{j}\right)=f\left(Z_{j}\right) / f\left(Z_{j}^{*}\right)$. Where $f\left(Z_{j}^{*}\right)$ is a benchmark objective value for instance $j$, which can be a lower bound or the best-known solution. As at each point $x$ each metaheuristic collects 25 samples for each instance, the final $y$-value in a point $x$ is the median of the instances' median sample. In these charts, the reference line $(y=1)$ represents the benchmark objective value $f\left(Z_{j}^{*}\right)$. In other words, the charts summarize the results for all instances and indicate, for each time point, how close or far the algorithm' median solutions are from the benchmark value, providing insights on the algorithm convergence rates.

Figure 6 plots the two best configurations with cooperative agents (thin lines) which were HM-ILS-1-30 with $(X, Y)=(1,30)$ and HM-ILS-4-30 with $(X, Y)=(4,30)$, the best configuration with independent agents (blue-thick line) which is HM-ILS-4-625 with $(X, Y)=(4,625)$ and the best sequential metaheuristic, the stand-alone ILS (red-thick line). These results indicate 


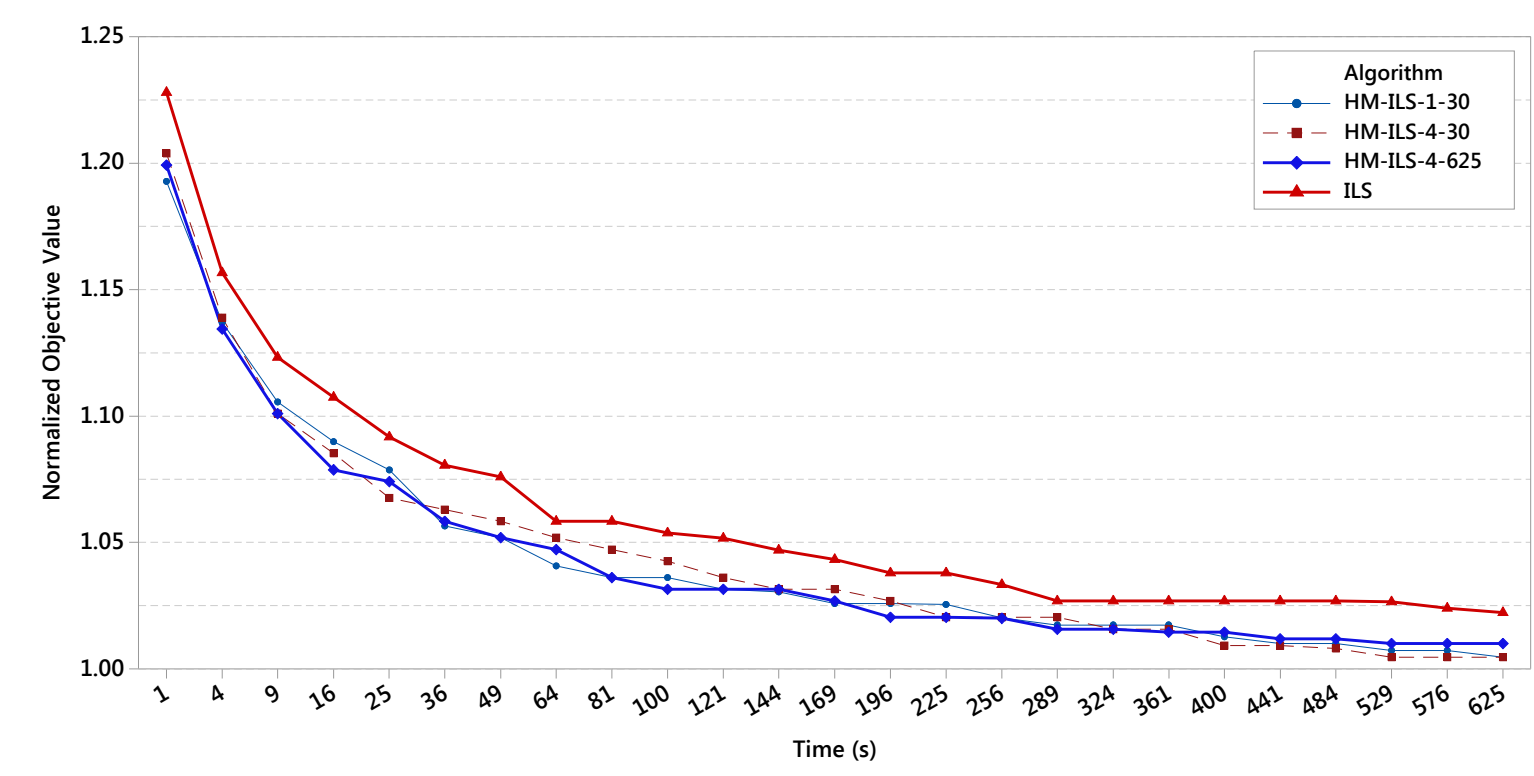

Figure 6 - Best results for CMB with homogeneous ILS agents (4 threads).

that parallel versions with homogeneous agents provide better quality solutions than sequential versions. We also observed this fact in the experiments using TS, SA and LAS as agents. In Figure 6, we also observe that after 400 seconds, configurations with cooperative ILS agents perform slightly better than their independent counterparts.

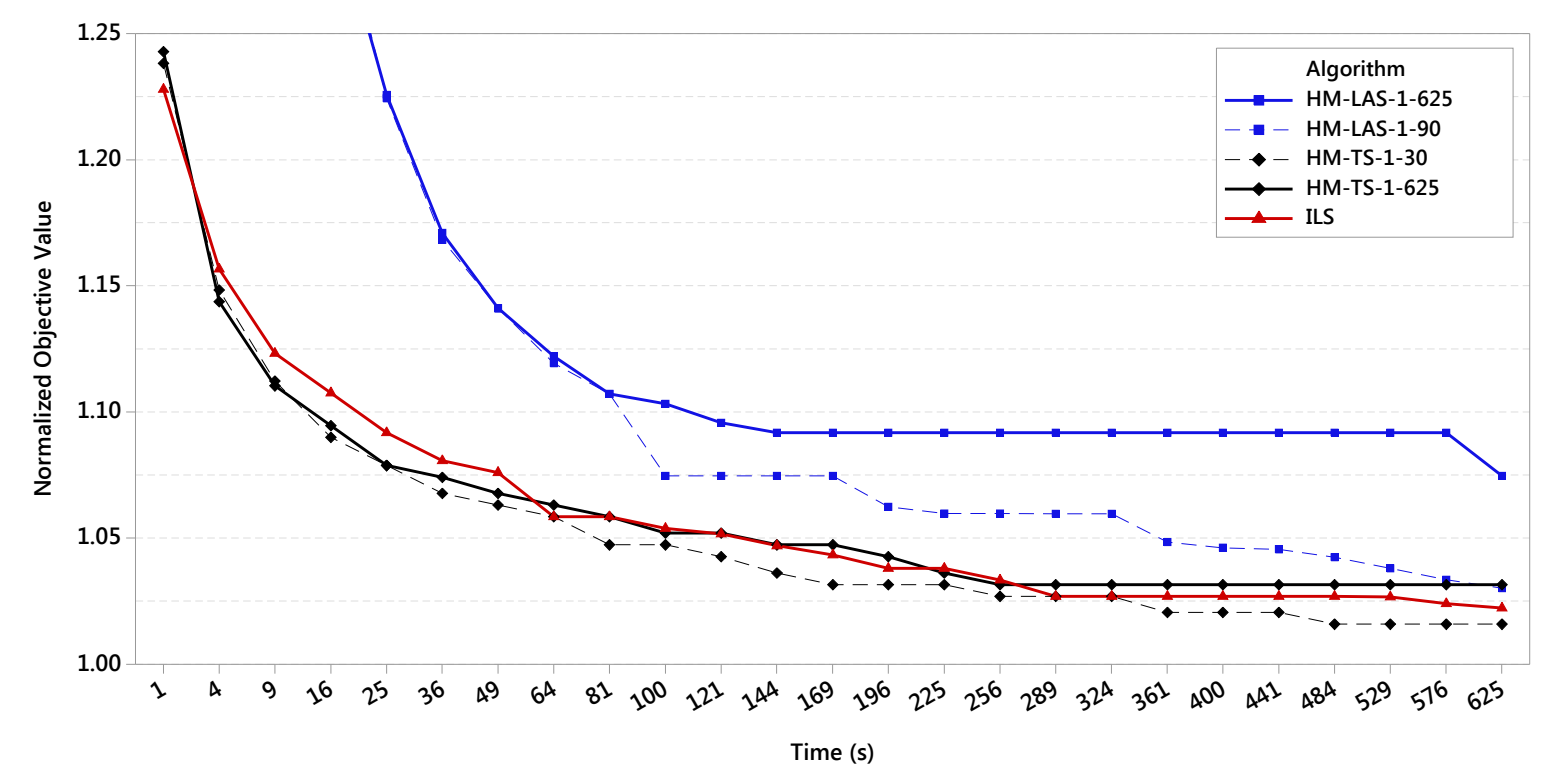

Figure 7 - Best results for CMB with homogeneous TS and LAS agents (4 threads).

Figure 7 plots the best configurations with homogeneous TS (black lines) and LAS (blue lines). We also plot the results of the stand-alone ILS in order to provide a common benchmark among different charts. The figure shows that configurations with cooperative agents (thin lines) perform significantly better than configurations with independent agents (thick lines). 
On the other hand, for SA, cooperative agents performed worse than independent agents. This is shown in Figure 8, where the thin line plots the best configuration with cooperative agents, the blue-thick line represents the best configuration with independent agents and the black-thick line is the best sequential SA. These results probably indicate that more sophisticated tuning procedures need to be used for the parallel SA, in order to balance the diversification provided by the parallel contribution and the natural diversification structure of the SA for high temperatures.

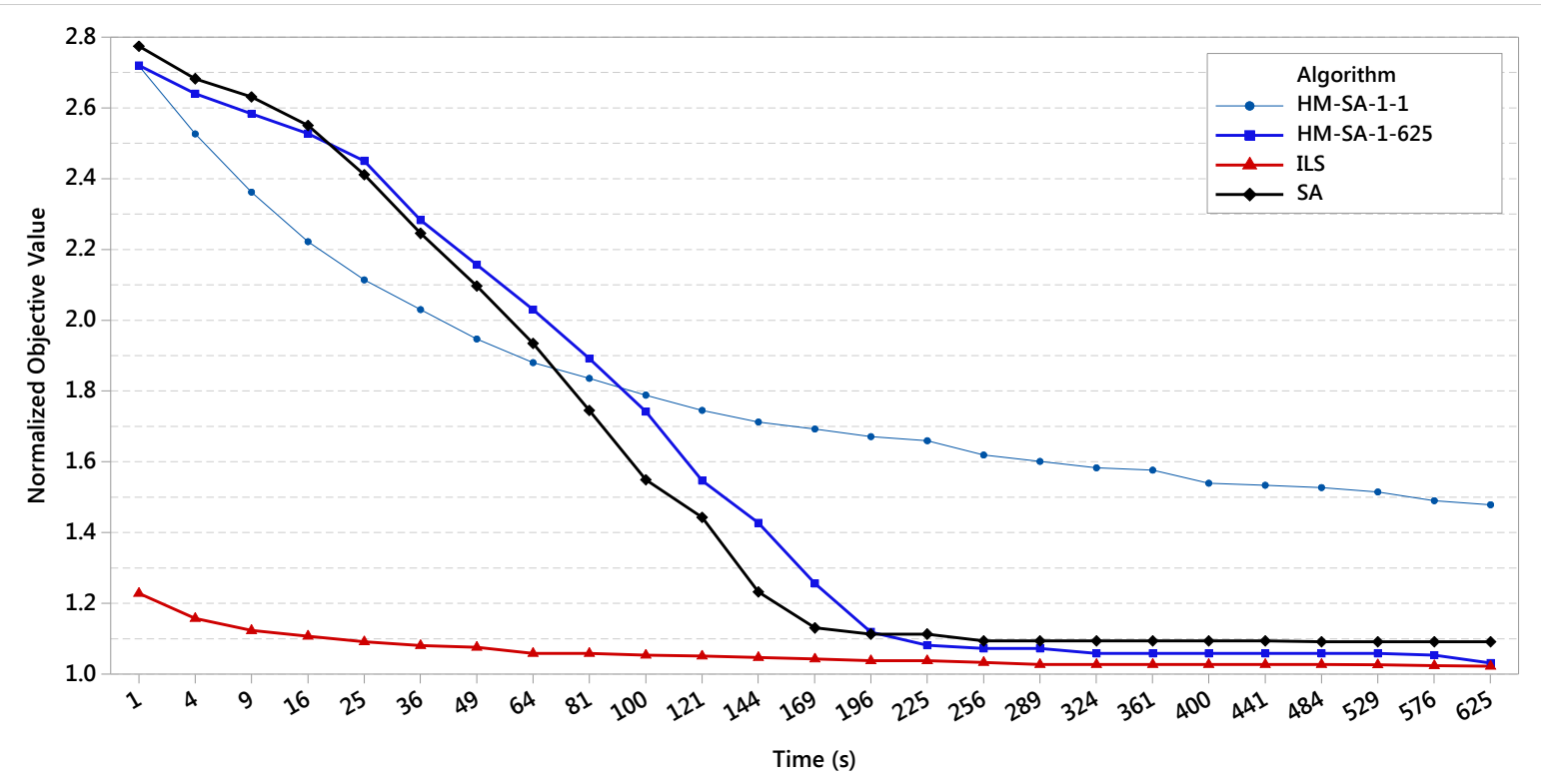

Figure 8 - Best results for CMB with homogeneous SA agents (4 threads).

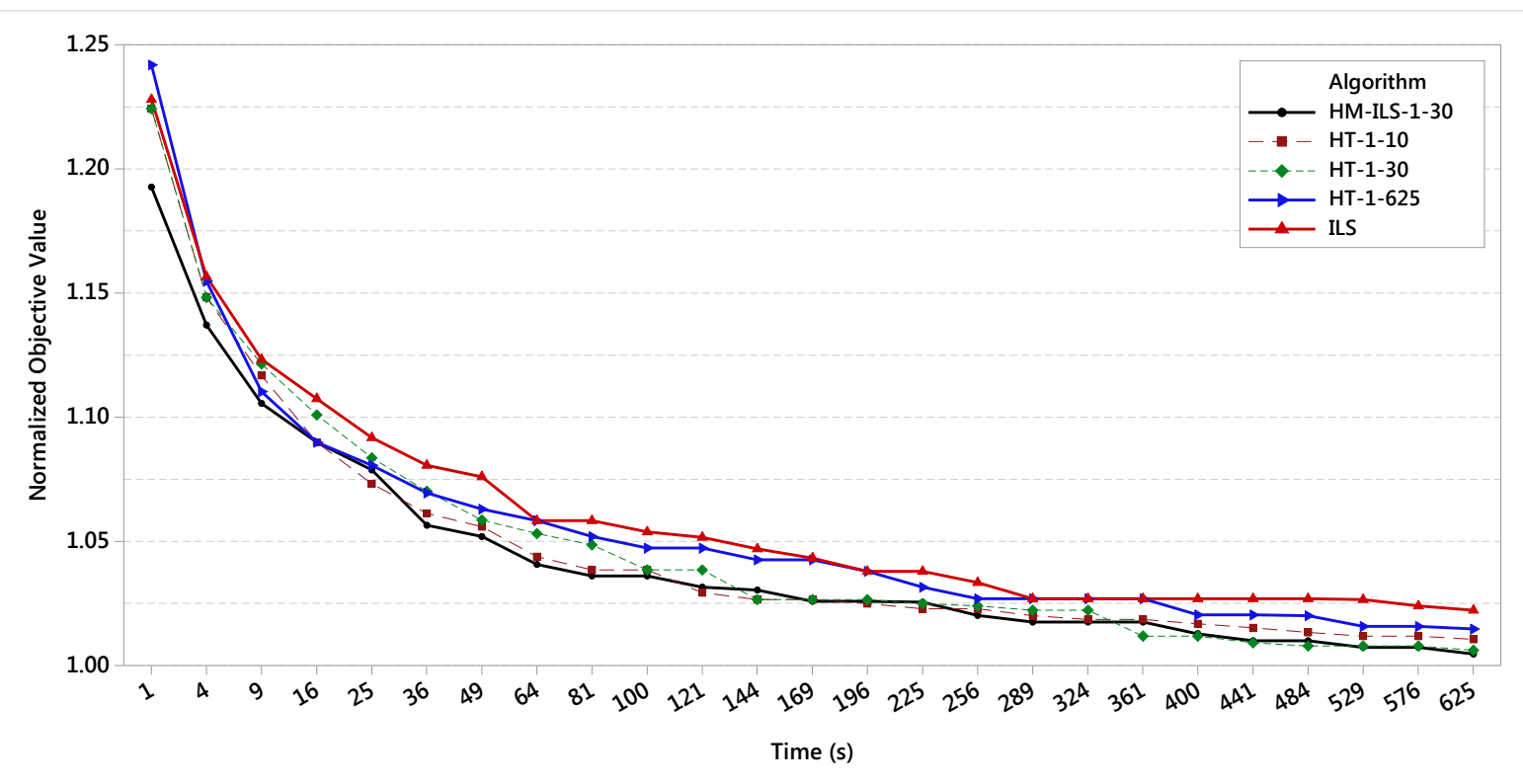

Figure 9 - Best results for CMB with heterogeneous agents (4 threads).

For CMB algorithms with heterogeneous (HT) agents, we tested the case in which there is one agent thread for each metaheuristic ILS, TS, SA and LAS. The TS agent was set with 
parameters $($ stSize, tbTime $)=(100,100)$, SA with $\left(T_{0}, \alpha\right.$, st Size, $\left.\varepsilon\right)=(100,0.9999,25,1)$ and LAS with $\left(l_{\text {sSize }}\right.$, stSize $)=(100,100)$. These parameters were chosen based on our sequential experiments and considering a trade-off between fast convergence and quality of solutions. As before, we tested configurations with central memory sizes $X=(1,4,8,16,32)$ and agents time $Y$ $=(1,2,5,10,30,90,315,625)$. In Figure 9, we plot the two best configurations with cooperative agents (thin lines), which were $(X, Y)=(1,10)$ and $(1,30)$, and the best configuration with independent agents (blue-thick line), which is $(X, Y)=(1,625)$. We observe that, configurations with cooperative agents (thin lines) perform better than configurations with independent agents (blue-thick line). Nevertheless, no heterogeneous configuration presented better results than the homogeneous ILS configuration HM-ILS-1-30, plotted in black-thick line in Figure 9.

Finally, it is interesting to see how all best versions used the central memory with a single solution, indicating a strong elitism characteristic.

\subsubsection{Results for diversification-intensification memory based algorithms}

In this section, we analyze a case of DIMB parallel metaheuristics in which the intensifier agents are several threads of ILS, which proved earlier to be the best sequential intensifier metaheuristic. As a diversifier agent, we use the LAS with parameters $(l s$ Size, stSize $)=(1000$, 25). Our preliminary tests showed that this metaheuristic provides diversified solutions to the diversification memory, because it quickly converges to local optima with quality near to $10 \%$ worse than the benchmark values, and it keeps moving to other solutions with the same quality.

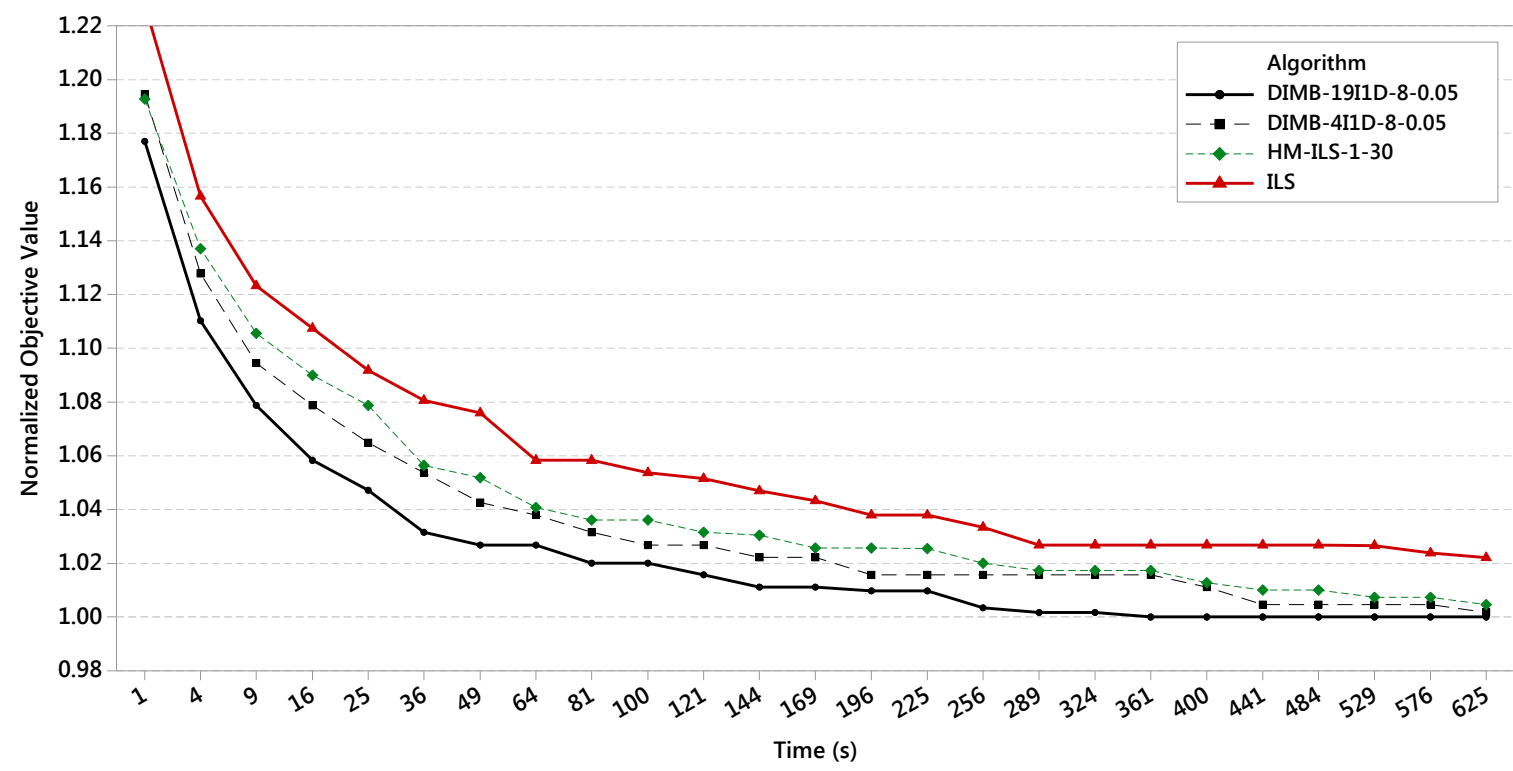

Figure 10 - Best results for DIMB parallel metaheuristics.

The DIMB algorithm was tested with four intensifiers (with a single solution in the intensification memory) and diversification memories with sizes $X_{D}=(1,4,8,16,32)$ combined with probabilities $\rho=(0.05,0.10,0.20,0.25,0.50)$. The best results were obtained with configu- 
ration $X_{D}=8$ and $\rho=0.05$. We also tested this configuration with nineteen intensifier threads. In Figure 10, we show these two configurations (algorithms DIMB-4I1D-8-0.05 and DIMB19I1D-8-0.05) and the best configuration of the CMB obtained earlier (algorithm HM-ILS-1-30). Comparing algorithms DIMB-4I1D-8-0.05 and HM-ILS-1-30, we observe that the scheme with diversification-intensification memories (DIMB) presents better performance than the scheme with central memory (CMB). Figure 10 also shows that increasing the number of threads has a beneficial effect on performance.

\subsubsection{Sequential versus parallel algorithms}

Figure 11 plots the distribution of the instances' median samples for parallel and sequential algorithms in the 34 instances from Table 15. The asterisks $\left(^{*}\right)$ represent outliers (best or worst solutions). This analysis provides some insights on the algorithms' robustness throughout the whole set of instances.

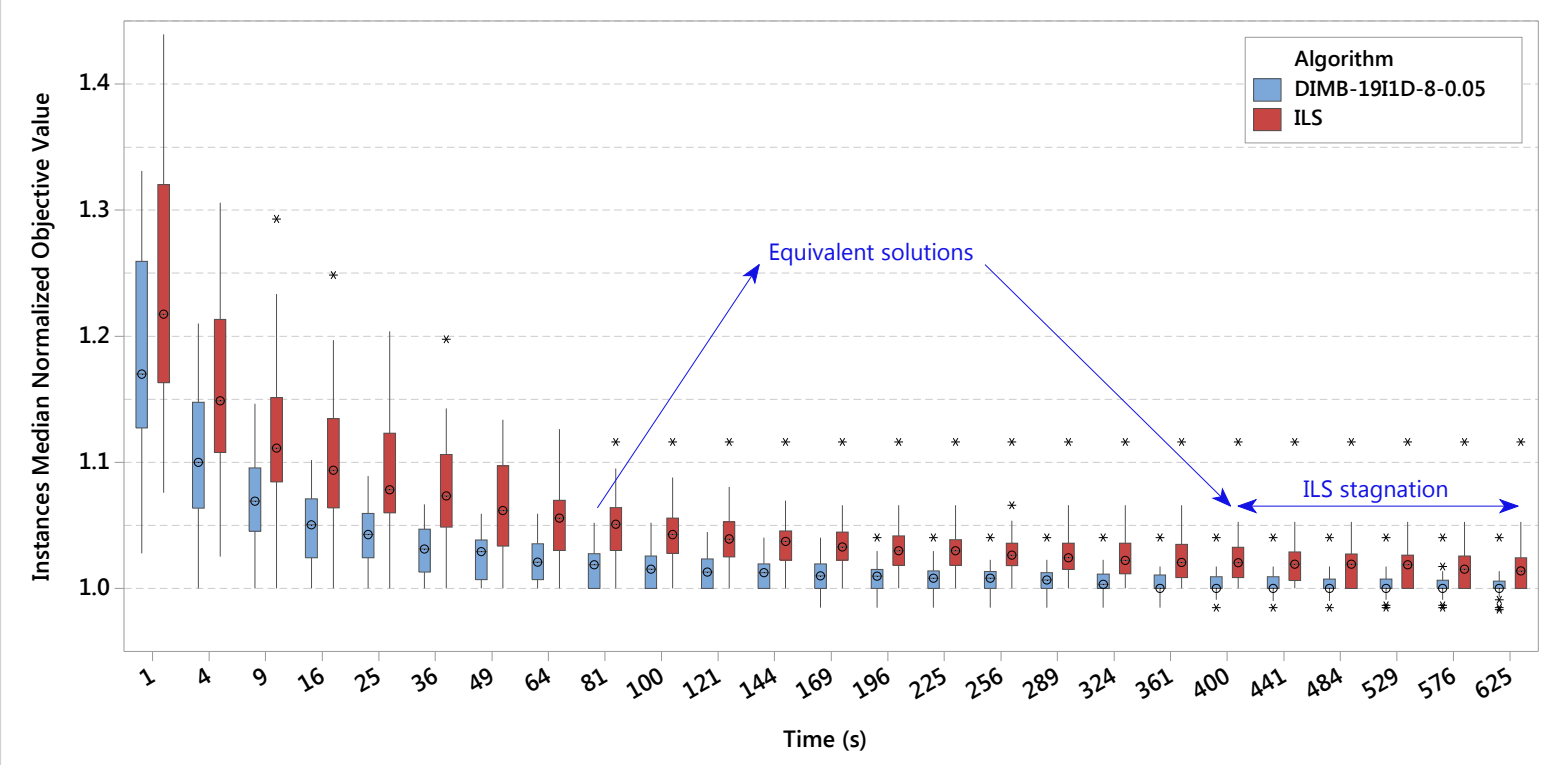

Figure 11 - Results of the best parallel algorithm DIMB-19I1D-8-0.05 compared to results of the best sequential ILS in all 34 instances from Table 15.

The best solutions of the sequential ILS were used as benchmark values. We observe that the parallel algorithm solutions after 81 seconds are statistically equivalent (supported by a Kruskal-Wallis test for the equality of medians) to those of the sequential ILS algorithm after 400 seconds, which is the time when the sequential ILS stagnates. Apart from being faster, the parallel algorithm is also more robust than the sequential version in all instances, as it presents less scattered distributions. 


\subsubsection{Comparisons with formulation CF2}

In this section, we compare the results of the best parallel algorithm DIMB-19I1D-8-0.05 with results of the best formulation CF2 in the 34 instances described in Table 15.

Table 24 - Results of algorithm DIMB-19I1D-8-0.05 compared to results of formulation CF2 in all 34 instances of Table 15.

\begin{tabular}{|c|c|c|c|c|c|c|c|}
\hline \multirow[b]{2}{*}{ ID } & \multirow[b]{2}{*}{ Best LB } & \multicolumn{2}{|c|}{ CF2-Cuts (3 h) } & \multicolumn{4}{|c|}{ DIMB-19I1D-8-0.05 (625 s) } \\
\hline & & UB & Gap & Median & Gap & Best & Gap \\
\hline 1 & 950 & 1040 & 8.65 & 970 & 2.06 & 950 & 0.00 \\
\hline 2 & 950 & 950 & 0.00 & 970 & 2.06 & 950 & 0.00 \\
\hline 3 & 1010 & 1040 & 2.88 & 1020 & 0.98 & 1010 & 0.00 \\
\hline 4 & 990 & 1900 & 47.89 & 1000 & 1.00 & 990 & 0.00 \\
\hline 5 & 870 & 870 & 0.00 & 870 & 0.00 & 870 & 0.00 \\
\hline 6 & 1080 & 1340 & 19.40 & 1100 & 1.82 & 1080 & 0.00 \\
\hline 7 & 1690 & 3525 & 52.06 & 1880 & 10.11 & 1810 & 6.63 \\
\hline 8 & 950 & 950 & 0.00 & 1030 & 7.77 & 980 & 3.06 \\
\hline 9 & 1100 & 1100 & 0.00 & 1170 & 5.98 & 1130 & 2.65 \\
\hline 10 & 420 & 420 & 0.00 & 420 & 0.00 & 420 & 0.00 \\
\hline 11 & 1400 & 1400 & 0.00 & 1530 & 8.50 & 1450 & 3.45 \\
\hline 12 & 1450 & 1450 & 0.00 & 1600 & 9.38 & 1530 & 5.23 \\
\hline 13 & 420 & 420 & 0.00 & 420 & 0.00 & 420 & 0.00 \\
\hline 14 & 1270 & 1270 & 0.00 & 1360 & 6.62 & 1300 & 2.31 \\
\hline 15 & 395 & 395 & 0.00 & 395 & 0.00 & 395 & 0.00 \\
\hline 16 & 640 & 640 & 0.00 & 650 & 1.54 & 640 & 0.00 \\
\hline 17 & 580 & 580 & 0.00 & 590 & 1.69 & 580 & 0.00 \\
\hline 18 & 460 & 460 & 0.00 & 460 & 0.00 & 460 & 0.00 \\
\hline 19 & 360 & 360 & 0.00 & 360 & 0.00 & 360 & 0.00 \\
\hline 20 & 470 & 470 & 0.00 & 470 & 0.00 & 470 & 0.00 \\
\hline 21 & 1450 & 1450 & 0.00 & 1550 & 6.45 & 1480 & 2.03 \\
\hline 22 & 1035 & 1035 & 0.00 & 1095 & 5.48 & 1055 & 1.90 \\
\hline 23 & 2505 & 17790 & 85.92 & 2790 & 10.22 & 2670 & 6.18 \\
\hline 24 & 2680 & 16825 & 84.07 & 2970 & 9.76 & 2860 & 6.29 \\
\hline 25 & 1315 & 1320 & 0.38 & 1400 & 6.07 & 1340 & 1.87 \\
\hline 26 & 750 & 750 & 0.00 & 780 & 3.85 & 750 & 0.00 \\
\hline 27 & 730 & 730 & 0.00 & 750 & 2.67 & 730 & 0.00 \\
\hline 28 & 1570 & 13420 & 88.30 & 1620 & 3.09 & 1590 & 1.26 \\
\hline 29 & 1570 & 2040 & 23.04 & 1620 & 3.09 & 1590 & 1.26 \\
\hline 30 & 1590 & 3500 & 54.57 & 1640 & 3.05 & 1600 & 0.63 \\
\hline 31 & 1570 & 3480 & 54.89 & 1640 & 4.27 & 1590 & 1.26 \\
\hline 32 & 1390 & 2185 & 36.38 & 1440 & 3.47 & 1410 & 1.42 \\
\hline 33 & 1410 & 2755 & 48.82 & 1460 & 3.42 & 1430 & 1.40 \\
\hline 34 & 1390 & 2205 & 36.96 & 1450 & 4.14 & 1410 & 1.42 \\
\hline Rar & & 20 & & 7 & & 26 & \\
\hline $\mathrm{Av}$ & & & 18.95 & & 3.78 & & 1.48 \\
\hline
\end{tabular}

The results are shown in Table 24. The table presents the median and best solutions (out of 25 runs) of the DIMB best configuration found earlier compared to the best solutions of formulation CF2. The best solutions are shown in bold font. The results show that although the program of $\mathrm{CF} 2$ has proven optimality for most of the tested instances, it did not find good quality solutions for 12 out of the 34 tested instances. Our parallel algorithm, on the other hand, provided optimal and near-optimal solutions in all instances, including those in which CF2 provided poor quality solutions. In average, the gaps (computed using the best lower bounds 
reported for these instances in Chapter 3, shown in column "Best LB") of the parallel algorithm are much smaller than those of formulation $\mathrm{CF} 2$.

\subsubsection{Comparisons with state-of-the-art algorithms}

In this section, we provide evidence that the proposed parallel algorithm DIMB-19I1D8-0.05 presents state-of-the-art results not only for problem HSTP-A but also for problems HSTP-B and HSTP-C, which have already been studied in the literature.

\subsubsection{Comparisons with methods for problem HSTP-B}

In this section, we report results of algorithm DIMB-19I1D-8-0.05 adapted to deal with problem HSTP-B. For these experiments, we consider the instances of Table 20, which have been used in the literature. The state-of-the-art algorithms for this problem are based on fix-and-optimize heuristics (DORNELES; ARAÚJO; BURIOL, 2014) and sequential ILS based metaheuristics (SAVINIEC et al., 2013). The fix-and-optimize heuristics were reported to find optimal solutions in each instance, on time limits within 10 to 60 minutes in a computer with processor Intel Core i5-2300 (2.8 GHz) and 4 GB of RAM, running Linux OS. The ILS metaheuristics were reported to find optimal solutions in each instance, on a time limit of 15 minutes in a computer with processor Intel Xeon E7-4860 (2.26 GHz) and $30 \mathrm{~GB}$ of RAM, running Windows OS.

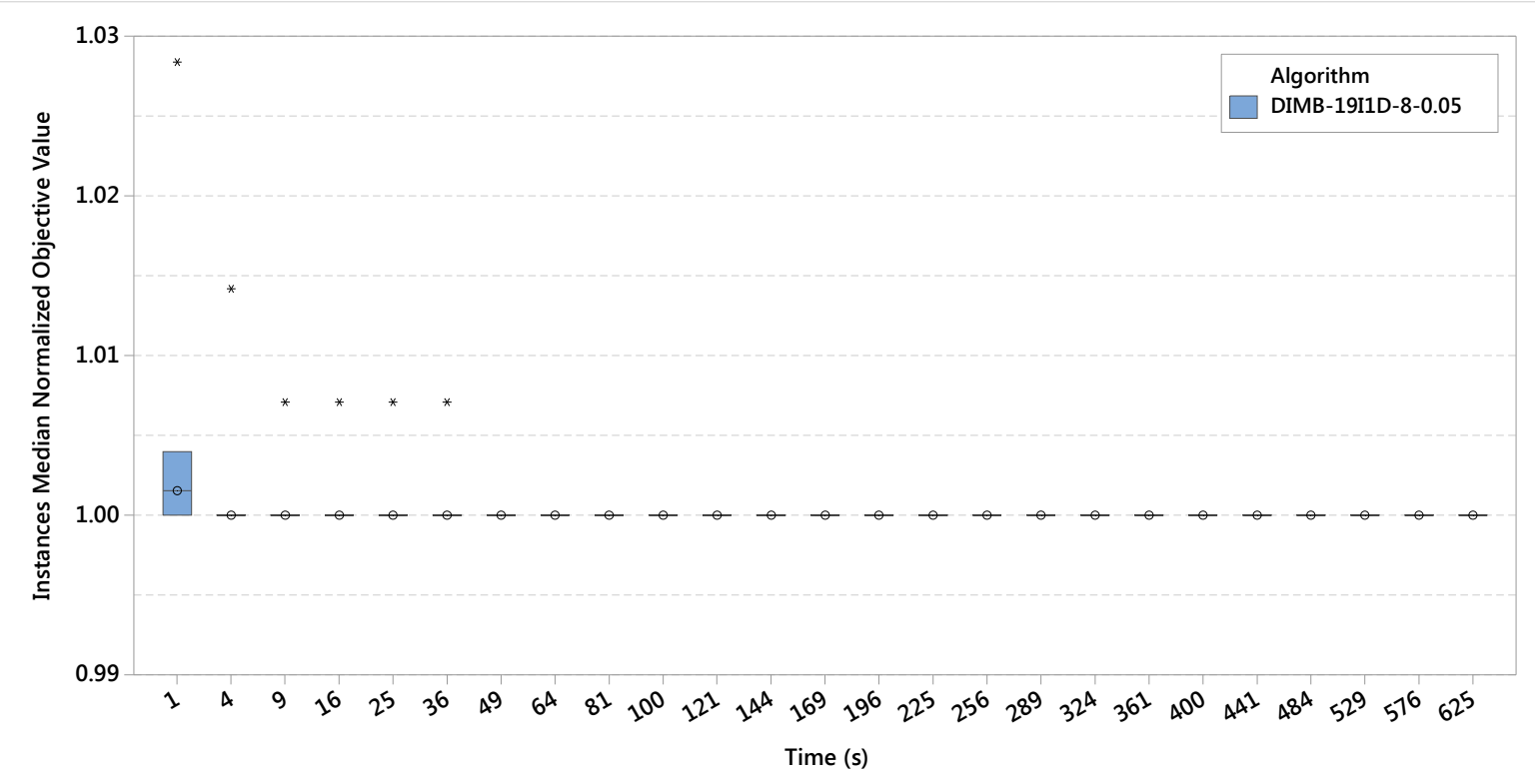

Figure 12 - Results of the algorithm DIMB-19I1D-8-0.05 on problem HSTP-B with instances of Table 20.

In Figure 12, we analyze the distribution of the instances' median samples generated by our parallel algorithm DIMB-19I1D-8-0.05. The instances optimal values described in Table 20 were used as benchmark values. We observe that, in the median case, the algorithm converges to optimal solutions in any instance within a time limit of 49 seconds. These results show that 
our algorithm is very robust to these instances and it is competitive with the state-of-the-art approaches.

\subsubsection{Comparisons with methods for problem HSTP-C}

Table 25 - Results of algorithm DIMB-19I1D-8-0.05 compared to results of GOAL on problem HSTP-C with instances of Table 15.

\begin{tabular}{|c|c|c|c|c|c|c|c|c|c|}
\hline \multirow[b]{2}{*}{ ID } & \multirow[b]{2}{*}{ Best LB } & \multicolumn{4}{|c|}{ DIMB-19I1D-8-0.05 (625 s) } & \multicolumn{4}{|c|}{ GOAL $(625 \mathrm{~s})$} \\
\hline & & Median & Gap & Best & Gap & Median & Gap & Best & Gap \\
\hline 1 & 699 & 710 & 1.55 & 700 & 0.14 & 730 & 4.25 & 716 & 2.37 \\
\hline 2 & 699 & 711 & 1.69 & 701 & 0.29 & 730 & 4.25 & 716 & 2.37 \\
\hline 3 & 742 & 749 & 0.93 & 743 & 0.13 & 770 & 3.64 & 762 & 2.62 \\
\hline 4 & 734 & 743 & 1.21 & 735 & 0.14 & 768 & 4.43 & 749 & 2.00 \\
\hline 5 & 631 & 631 & 0.00 & 631 & 0.00 & 648 & 2.62 & 642 & 1.71 \\
\hline 6 & 772 & 777 & 0.64 & 772 & 0.00 & 814 & 5.16 & 800 & 3.50 \\
\hline 7 & 1212 & 1270 & 4.57 & 1239 & 2.18 & 1312 & 7.62 & 1297 & 6.55 \\
\hline 8 & 675 & 698 & 3.30 & 686 & 1.60 & 682 & 1.03 & 676 & 0.15 \\
\hline 9 & 807 & 827 & 2.42 & 814 & 0.86 & 838 & 3.70 & 829 & 2.65 \\
\hline 10 & 298 & 298 & 0.00 & 298 & 0.00 & 300 & 0.67 & 298 & 0.00 \\
\hline 11 & 961 & 1005 & 4.38 & 985 & 2.44 & 1052 & 8.65 & 1024 & 6.15 \\
\hline 12 & 1018 & 1044 & 2.49 & 1030 & 1.17 & 1048 & 2.86 & 1034 & 1.55 \\
\hline 13 & 273 & 273 & 0.00 & 273 & 0.00 & 273 & 0.00 & 273 & 0.00 \\
\hline 14 & 933 & 954 & 2.20 & 939 & 0.64 & 971 & 3.91 & 949 & 1.69 \\
\hline 15 & \multicolumn{9}{|c|}{ Infeasible } \\
\hline 16 & 481 & 484 & 0.62 & 481 & 0.00 & 498 & 3.41 & 490 & 1.84 \\
\hline 17 & 457 & 461 & 0.87 & 457 & 0.00 & 468 & 2.35 & 462 & 1.08 \\
\hline 18 & 319 & 319 & 0.00 & 319 & 0.00 & 324 & 1.54 & 319 & 0.00 \\
\hline 19 & 254 & 254 & 0.00 & 254 & 0.00 & 254 & 0.00 & 254 & 0.00 \\
\hline 20 & 325 & 325 & 0.00 & 325 & 0.00 & 328 & 0.91 & 325 & 0.00 \\
\hline 21 & 1050 & 1074 & 2.23 & 1058 & 0.76 & 1107 & 5.15 & 1091 & 3.76 \\
\hline 22 & \multicolumn{9}{|c|}{ Infeasible } \\
\hline 23 & 1813 & 1910 & 5.08 & 1867 & 2.89 & 2013 & 9.94 & 1971 & 8.02 \\
\hline 24 & 1988 & 2079 & 4.38 & 2038 & 2.45 & 2171 & 8.43 & 2128 & 6.58 \\
\hline 25 & 912 & 944 & 3.39 & 926 & 1.51 & 987 & 7.60 & 966 & 5.59 \\
\hline 26 & 570 & 576 & 1.04 & 570 & 0.00 & 603 & 5.47 & 584 & 2.40 \\
\hline 27 & 552 & 556 & 0.72 & 552 & 0.00 & 566 & 2.47 & 560 & 1.43 \\
\hline 28 & 1124 & 1150 & 2.26 & 1127 & 0.27 & 1183 & 4.99 & 1167 & 3.68 \\
\hline 29 & 1116 & 1145 & 2.53 & 1127 & 0.98 & 1187 & 5.98 & 1168 & 4.45 \\
\hline 30 & 1133 & 1164 & 2.66 & 1142 & 0.79 & 1203 & 5.82 & 1175 & 3.57 \\
\hline 31 & 1127 & 1165 & 3.26 & 1137 & 0.88 & 1195 & 5.69 & 1181 & 4.57 \\
\hline 32 & 1014 & 1043 & 2.78 & 1024 & 0.98 & 1069 & 5.14 & 1063 & 4.61 \\
\hline 33 & 1018 & 1047 & 2.77 & 1034 & 1.55 & 1078 & 5.57 & 1060 & 3.96 \\
\hline 34 & 1005 & 1041 & 3.46 & 1015 & 0.99 & 1070 & 6.07 & 1056 & 4.83 \\
\hline$\overline{A v g}$ & & & 1.98 & & 0.74 & & 4.35 & & 2.93 \\
\hline
\end{tabular}

Lastly, we compare our parallel algorithm DIMB-19I1D-8-0.05 with the heuristic solver GOAL (FONSECA et al., 2016) on problem HSTP-C. This solver was the winner of the ITC2011. It provides a pool of methods to solve timetabling problems from different countries. The latest version of this solver ${ }^{1}$ (FONSECA; SANTOS; CARRANO, 2016), which was used in this comparison, is a three-phase approach. At the first phase, the KHE software library (KINGSTON, 2015) is employed to obtain a starting solution. This solution is refined by a

\footnotetext{
1 Available in <http://www.goal.ufop.br/software/hstt/>
} 
parallel VNS metaheuristic at the second phase, and lastly, the output of VNS is further improved with fix-and-optimize MIP heuristics.

Table 25 presents the results of 25 runs of both algorithms on instances of Table 15 . Both algorithms were set with 20 threads and a time limit of 625 seconds. As suggested by the authors of GOAL, additional parameters of their solver were set to default values (alg-timelimit $=62$, form-timelimit $=62$, initial-soln $=\mathrm{KHE}$, algorithm $=$ SVNS, formulation $=$ FIXOPT, formfixopt-nresources $=5$ and formfixopt-optinarow $=5$ ). In Table 25, bold font highlights the best solutions found. The results show that our algorithm outperforms GOAL, both in terms of median and best solutions. Our algorithm only performed worse than GOAL in instance 8 . The probable reason is that this instance has a large number of teachers' unavailable periods, in which it is expected that MIP neighborhoods (such as those employed in GOAL) will perform better than conventional neighborhood approaches (such as the one employed in our algorithm). We also note that the average gap (computed using the best lower bounds reported for problem HSTP-C in Chapter 3, shown in column "Best LB") of our median solutions (1.98\%) is smaller than the average gap of the best solutions of GOAL (2.93\%).

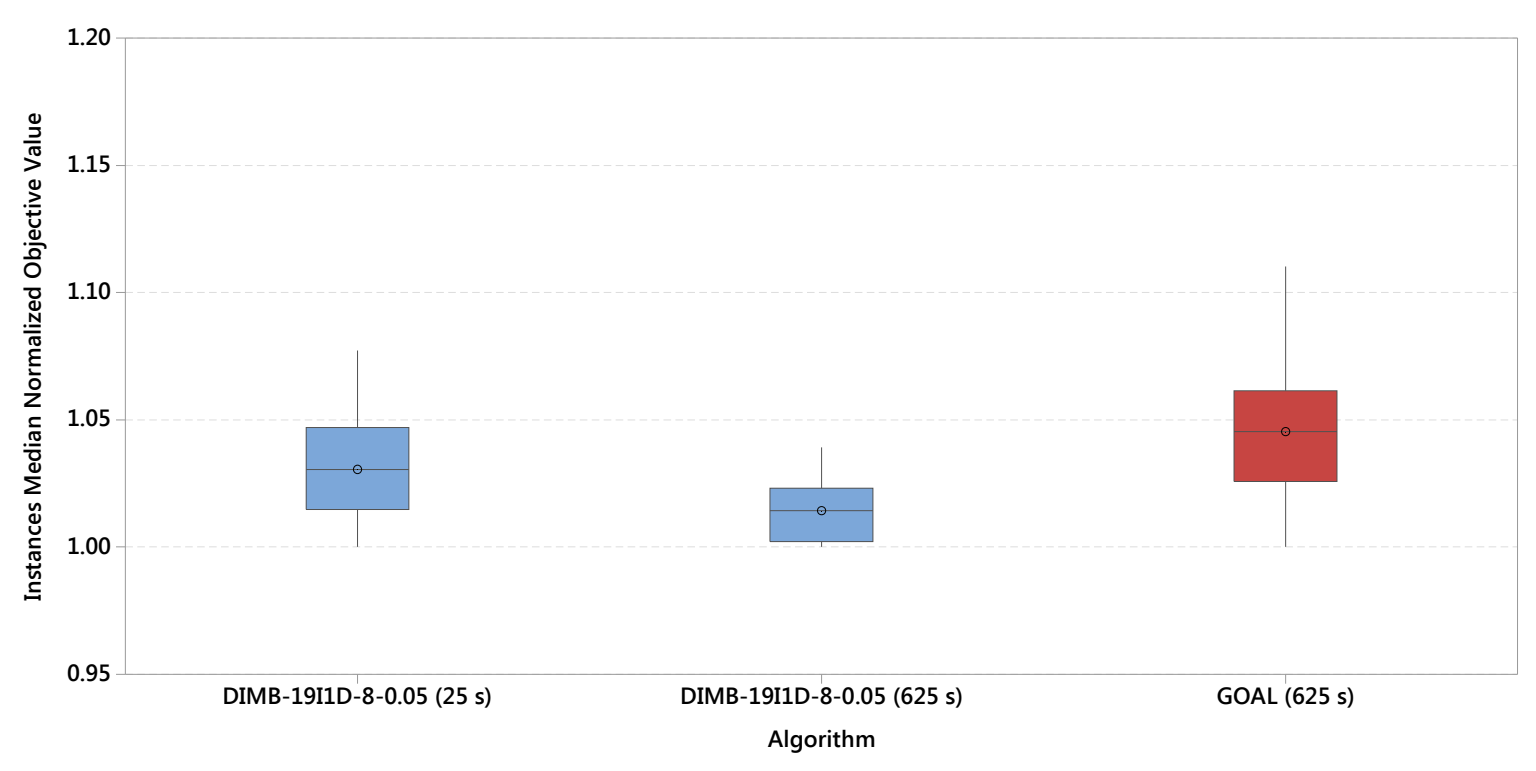

Figure 13 - Results of algorithm DIMB-19I1D-8-0.05 after 25 and 625 seconds compared to results of GOAL after 625 seconds on instances of Table 15.

In Figure 13, we focus on the computational time spent by both algorithms. The chart shows the distribution of the instances' median solutions generated by DIMB-19I1D-8-0.05 after 25 and 625 seconds and the results of GOAL after 625 seconds. A Kruskal-Wallis test for the equality of medians was run and indicated that the solutions generated by our parallel algorithm after 25 seconds are of better quality than those of GOAL after 625 seconds. 


\subsection{Final remarks}

In this chapter, we conducted an extensive study with parallel trajectory-based metaheuristics for high school timetabling problems. Our study investigated several aspects related to the design of such algorithms. In particular, we analyzed the homogeneity/heterogeneity of agents, the influence of intensification and diversification memories, and the frequency of information exchange among agents.

Our most efficient algorithm was obtained with the inclusion of a diversification memory, a very elitist central memory (only the best current solution was kept), short agents time which favored the exchange of information among threads with frequent restarts and multiple copies of the same metaheuristic agent (an iterated local search procedure). This configuration was able to consistently obtain good quality solutions for problem HSTP-A and also outperformed state-of-the-art algorithms on the other two variants, HSTP-B and HSTP-C. These last variants ignored specific requisites or considered some of them as hard instead of soft requisites.

These results suggest that the proposed method is efficient and robust concerning the enabling/disabling of constraints. This latter characteristic is particularly envisaged for HSTP algorithms since practical problems usually contain specific requisites. Our exploratory study limited itself to simulations with only four metaheuristic agents in which the threads had static execution time. Further research is aimed at exploring more flexible frameworks, in which other metaheuristics can be included and the agents' execution time can be self-calibrated according to the search history. 
CHAPTER

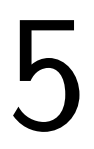

(1)

\section{PARALLEL METAHEURISTIC WITH COLUMN GENERATION}

In the previous chapters, we have investigated mixed-integer programming models and parallel metaheuristic based algorithms for high school timetabling problems. As shown, the computation of solutions and lower bounds is time-consuming. In this chapter, we propose a DIMB algorithm that exploits column generation into a cooperative parallel framework to simultaneously generate high-quality solutions and lower bounds for high school timetabling problems. We explain this algorithm in Section 5.1 and assess its performance in Section 5.2.

\subsection{A DIMB algorithm with column generation}

The proposed DIMB algorithm with column generation (DIMB-CG) is an extension of the DIMB framework described in Section 4.2.2. It is illustrated in Figure 14 and has the following components:

- Three shared memories: a diversification memory of solutions, an intensification memory of solutions, and a pool of columns.

- Four communication buffers: a buffer of input solutions, a buffer of output solutions, a buffer of dual values, and a buffer of columns.

- A set of algorithm agents: a column generation procedure (CG), a constructive column generation based heuristic (CCGH), a manager procedure, a diversifier procedure, and $N$ intensifier metaheuristics.

Algorithm DIMB-CG is based on three main ideas. First, it incorporates a column generation into a parallel framework to compute lower bounds. Second, it exploits the generated columns to build new feasible solutions that are used to diversify the search. Third, it takes 
advantage of cooperative parallel metaheuristics to improve the constructed solutions. Below, we explain the details of each component of this algorithm.

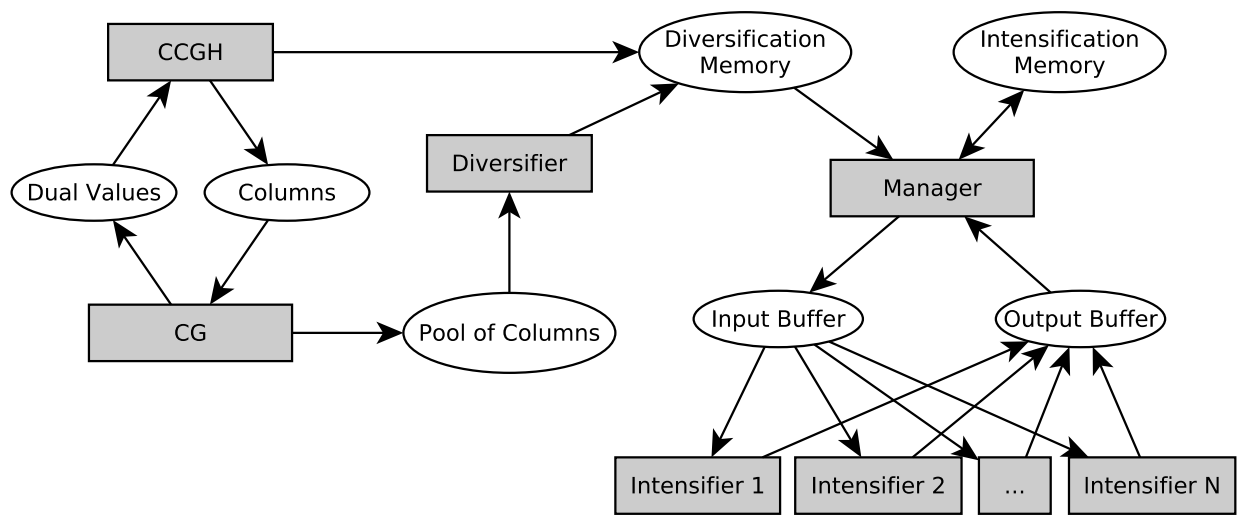

Figure 14 - An illustration of the algorithm DIMB-CG.

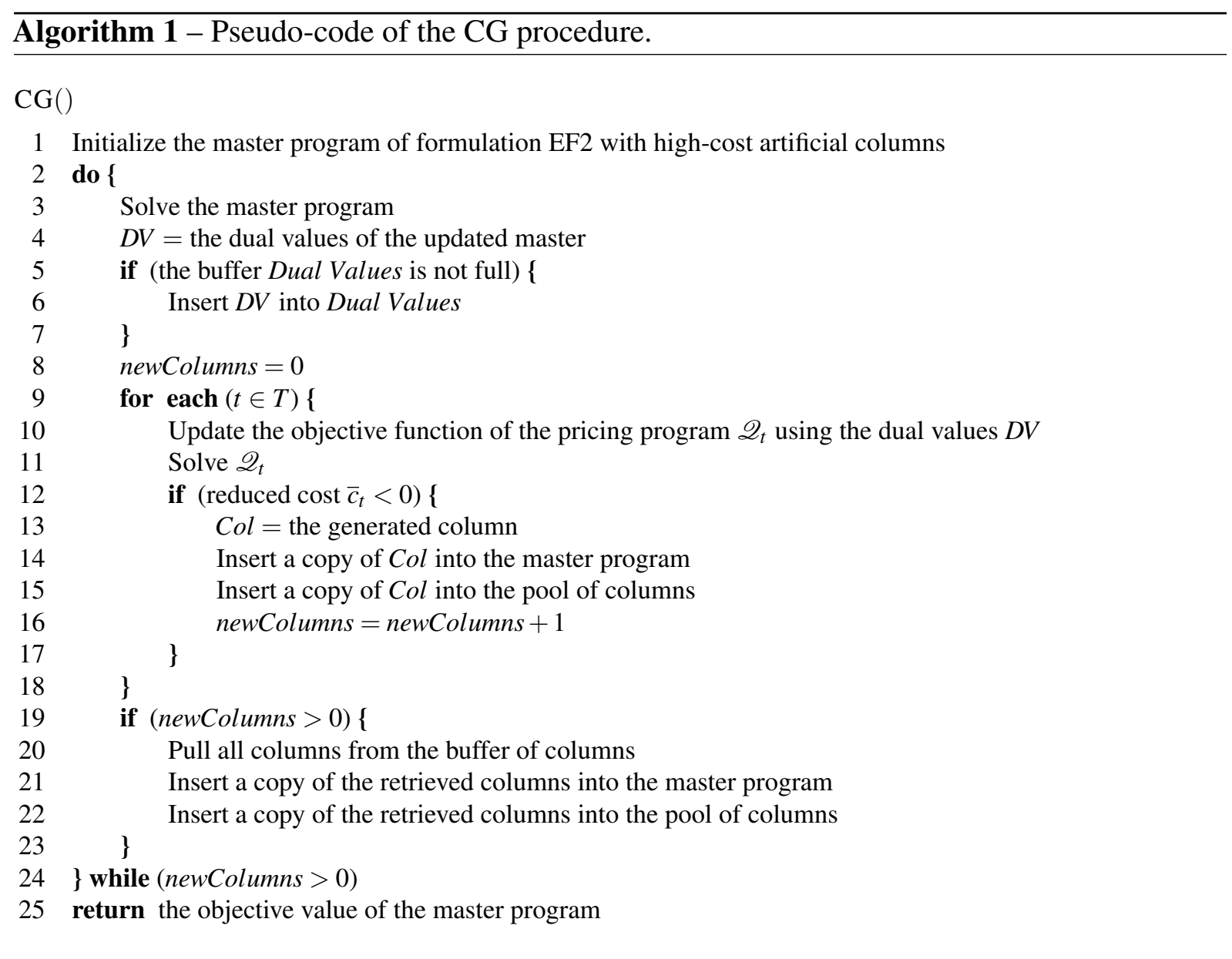

\section{Memories and buffers}

The diversification memory keeps a set of non-elite solutions that are used to diversify the search, while the intensification memory maintains a set of elite solutions which are used to 
intensify the search. Both memories have static sizes. The pool of columns is a dynamic memory which stores all columns generated by the column generation and the constructive column generation based heuristic. Lastly, the buffers are static and act as communication mechanisms.

\section{Column generation procedure}

The column generation procedure (CG), shown in Algorithm 1, employs formulation $\mathrm{EF} 2$ described in Section 3.2.2. It is a standard column generation algorithm, except that at each iteration, it interacts with the constructive heuristic CCGH (Algorithm 2) by sending dual values (lines 5 to 7 ) and receiving columns generated by the constructive heuristic (line 20). Also, the CG procedure stores a copy of all produced columns into the pool of columns (lines 15 and 22), so that the columns can be further exploited by the diversifier agent (Algorithm 4).

\section{Timetable encodings}

The remaining agents handle two types of timetable encoding. The timetable types $X$ and $Z$, that are illustrated in Figure 15. Both of them are based on the data structure proposed in Section A.1. In timetable type $X$ (Figure 15a), there is an array of timeslots, and each timeslot points to an array of pointers. These pointers point to requirement tuples, which represent the requirements assigned to teachers. In timetable type $Z$ (Figure 15b), each timeslot points to an array of pointers, that point to requirement tuples assigned to classes. In the remainder of this chapter, to improve the presentation, instead of showing a pointer to a requirement tuple itself, we will display only the teacher ID or class ID belonging to the tuple being pointed by the pointer.

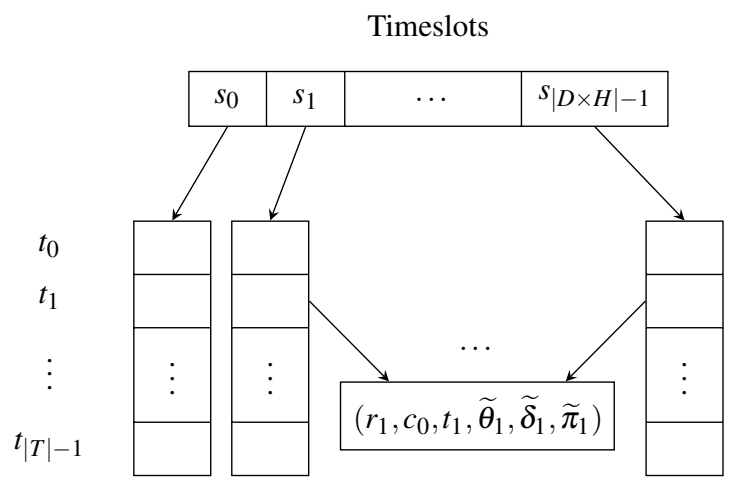

(a) Timetable type $X$ : teachers view.

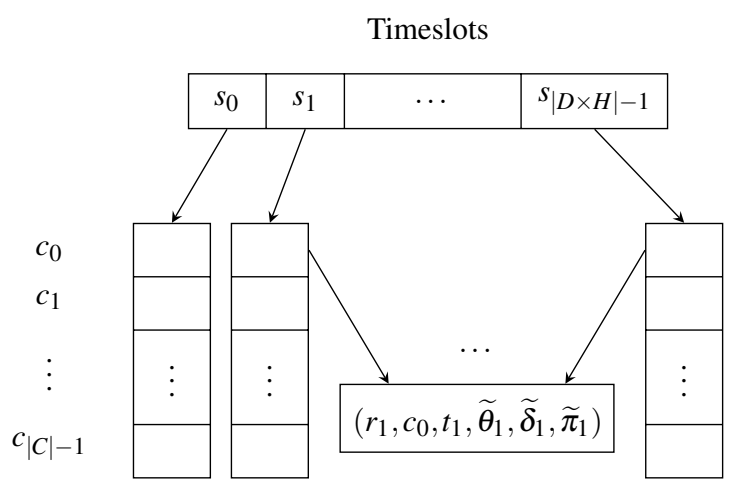

(b) Timetable type $Z$ : classes view.

Figure 15 - Timetable encodings.

Neighboring solutions are generated via the TQ operator (SAVINIEC; CONSTANTINO, 2017). This operator identifies chains of two-swap moves that must be performed together so that new clashes in classes or teachers' schedules are avoided. Depending on the timetable encoding used, a two-swap move consists in exchanging the requirement tuples of a class $c \in C$, or teacher 
Timeslots

\begin{tabular}{|c|c|c|c|c|c|}
\hline & $s_{0}$ & $s_{1}$ & $s_{2}$ & $s_{3}$ & $S_{4}$ \\
\hline a column of teacher $t_{0}$ & 1 & 2 & & 1 & 2 \\
\hline a column of teacher $t_{1}$ & 2 & 1 & 3 & 3 & \\
\hline a column of teacher $t_{2}$ & 4 & & 0 & 2 & 3 \\
\hline a column of teacher $t_{3}$ & & 3 & 4 & 4 & 0 \\
\hline a column of teacher $t_{4}$ & 0 & 0 & 2 & & 4 \\
\hline a column of teacher $t_{5}$ & 3 & 4 & 1 & 0 & 1 \\
\hline
\end{tabular}

(a) A set of non-conflicting columns considering a toy timetable with five weekly timeslots. Two columns are conflict-free if they have no clashes in classes' schedules.

Timeslots

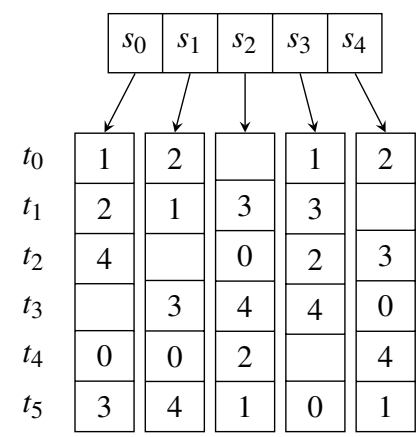

(c) Example of a clash-free timetable $X$ constructed with information from the columns of Figure 16a.

Timeslots

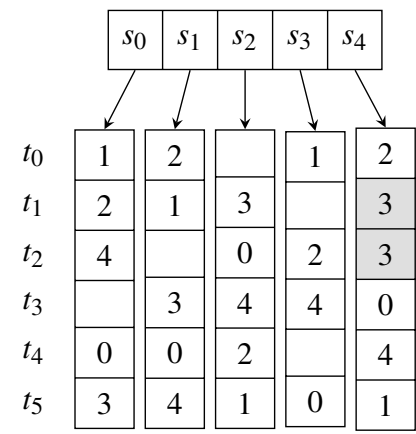

(e) Example of a non-clash-free timetable $X$ constructed with information from the columns of Figure 16b.
Timeslots

$\begin{array}{lllll}s_{0} & s_{1} & s_{2} & s_{3} & s_{4}\end{array}$

\begin{tabular}{|c|c|c|c|c|c|}
\hline a column of teacher $t_{0}$ & 1 & 2 & & 1 & 2 \\
\hline a column of teacher $t_{1}$ & 2 & 1 & 3 & & 3 \\
\hline a column of teacher $t_{2}$ & 4 & & 0 & 2 & 3 \\
\hline a column of teacher $t_{3}$ & & 3 & 4 & 4 & 0 \\
\hline a column of teacher $t_{4}$ & 0 & 0 & 2 & & 4 \\
\hline column of teacher $t_{5}$ & 3 & 4 & 1 & 0 & 1 \\
\hline
\end{tabular}

(b) A set of conflicting columns. Note that there is a clash in the schedule of class $c_{3}$, it is assigned to meet two teachers $\left(t_{1}\right.$ and $\left.t_{2}\right)$ in timeslot $s_{4}$.
Timeslots

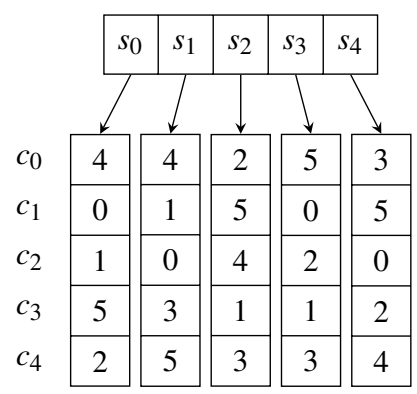

(d) Example of a clash-free timetable $Z$ constructed with information from the columns of Figure 16a.

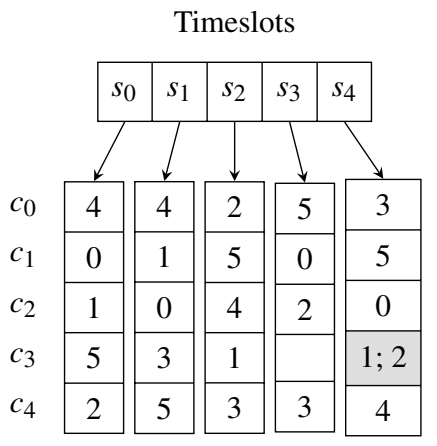

(f) Example of a timetable $Z$ that cannot be constructed with information from the columns of Figure 16b, because an array cell cannot point to two tuples at the same time.

Figure 16 - Construction of solutions with information provided by columns.

$t \in T$, that are assigned to two different timeslots. Chains of two-swap moves are identified by conflict graphs, similarly as made in Section A.2. 
Timetable solutions are evaluated by the objective function (A.1), described in Section A.4. For a timetable solution of type $Z$, hard requisites 1 and 2 are left out of the objective function, because they are maintained by the TQ moves and the timetable encoding. The same is valid for hard requisites 1 and 3 in a timetable solution of type $X$. For both timetable encodings, all other requisites are penalized in the objective function according to the problem being handled.

In Figure 16, we explain how the columns of the column generation are interpreted and used along with these timetable encodings to construct solutions. As shown in Figures 16a and 16 b, a column provided by a pricing subproblem $\mathscr{Q}_{t}$ (see Section 3.2.2.1) is a complete weekly schedule for a teacher $t \in T$. Columns are also represented by requirement tuples, but we will show only the associated classes to make the presentation easier. Supposing that a subset of columns is available for each teacher, it is sufficient to choose one column at random for each teacher to build an initial solution. Two situations might arise. On the one hand, if the chosen columns are conflict-free columns, as in Figure 16a, then we are able to construct solutions with both timetable encodings, as shown in Figures $16 \mathrm{c}$ and 16d. On the other hand, if the chosen columns have conflicting columns, as in Figure 16b, then we can still build (infeasible) solutions with timetable encoding $X$, as shown in Figure 16e. However, we cannot produce solutions with timetable encoding $Z$, as exemplified in Figure $16 \mathrm{f}$.

\section{Constructive column generation heuristic}

The constructive column generation heuristic (CCGH) is shown in Algorithm 2. This heuristic tries to construct a feasible solution (lines 6 to 22) every time a new vector of dual values is received from the CG algorithm (line 5). If it succeeds, then the constructed solution is further improved by the ILS described in Algorithm 3 (line 24), a copy of its columns is sent back to the CG algorithm (line 25), and the final solution is sent to the diversification memory (lines 26 to 30). The ILS in Algorithm 3 is similar to the one described in Algorithm 8 of Section A.5.

To create feasible solutions, we use two strategies. First, we solve each subproblem by fixing some of its variables based on schedules already defined in previous subproblems (SANTOS et al., 2011) (lines 8 to 15). This strategy is expected to fail, because, in the last subproblems, most of the variables will be fixed, which might result in infeasible subproblems. If it fails at a given subproblem with a partial solution $Z_{p}$, then we employ the second strategy, which consists in solving a proximity problem (FISCHETTI; MONACI, 2014) to find a complete feasible solution $Z$ that minimizes the Hamming distance to $Z_{p}$ (lines 19 to 21).

Now, we explain how the variables of a subproblem $\mathscr{Q}_{t}$ are preprocessed in line 10 , and how the proximity problem is created and solved in line 20.

In Figure 17, we illustrate the preprocessing of variables. After solving the subproblems of teachers $t_{0}$ to $t_{3}$, we can forbid class $c_{2}$ of being assigned to teacher $t_{4}$ at timeslots $s_{0}, s_{1}, s_{3}$ 


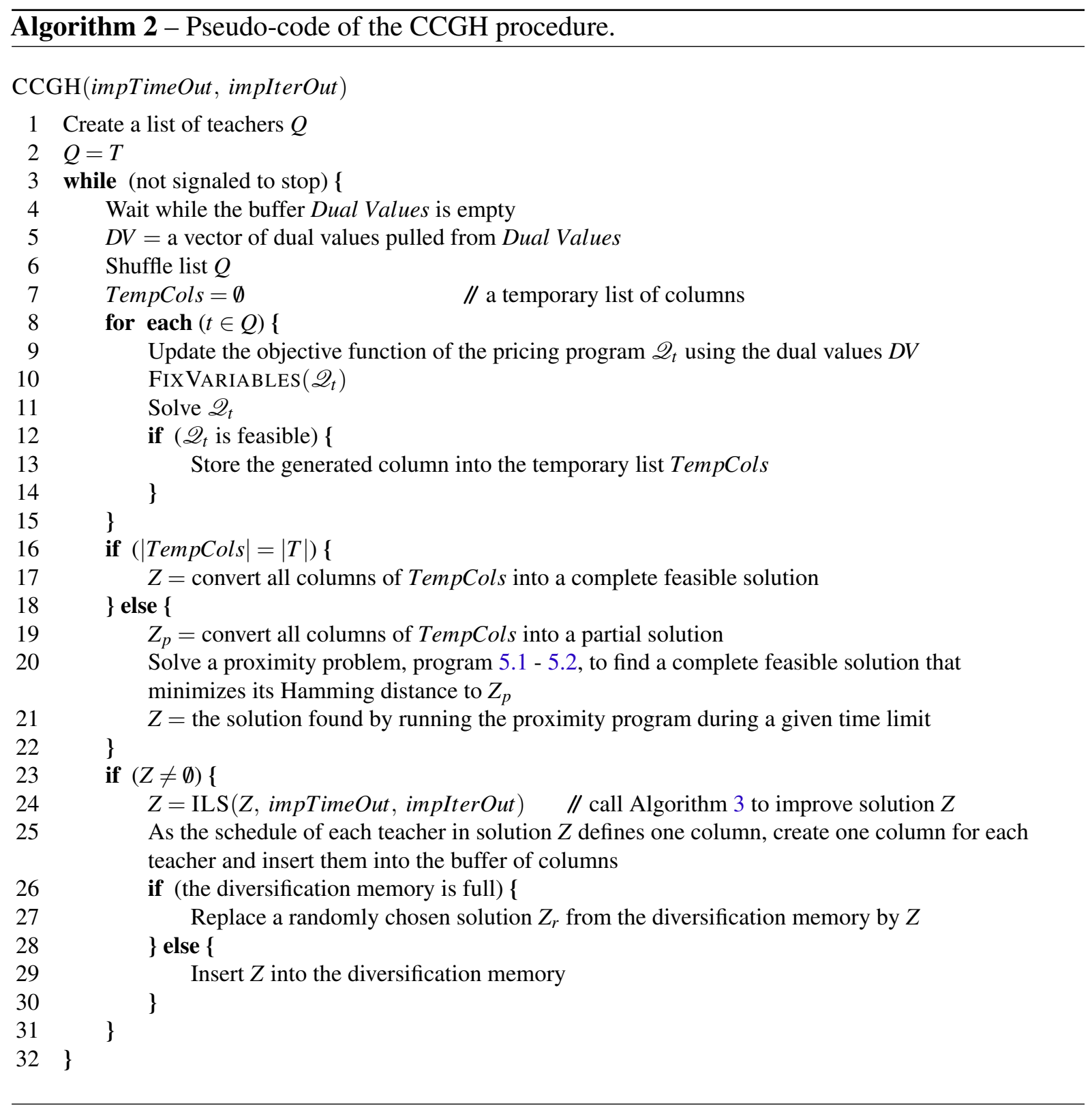

and $s_{4}$, because it is already assigned to the same timeslots in the previous subproblems. The same idea is employed for the remaining classes $\left(c_{0}\right.$ and $\left.c_{4}\right)$ that have meetings with teacher $t_{4}$, shown between parenthesis in Figure 17. We apply this preprocessing strategy before solving each subproblem in line 11 of the CCGH heuristic.

Now, let us suppose that teacher $t_{4}$ is unavailable at timeslot $s_{2}$ in the example of Figure 17. In this case, the subproblem is infeasible, because class $c_{2}$ cannot be assigned to any timeslot. When we reach such infeasibility, we solve the proximity problem, in line 20, considering formulation CF1 proposed in Section 3.1.1.

Let $J$ be the subset of variables $x_{r d h}$, in model CF1, which belong to teachers with schedules already fixed. For example, teachers $t_{0}, t_{1}, t_{2}$ and $t_{3}$ in Figure 17. Based on these fixed schedules, let $J_{0} \subset J$ be the subset of variables that have values set to zero, and $J_{1} \subset J$ be the subset of variables that have values set to one. The proximity problem can thus be written as: 


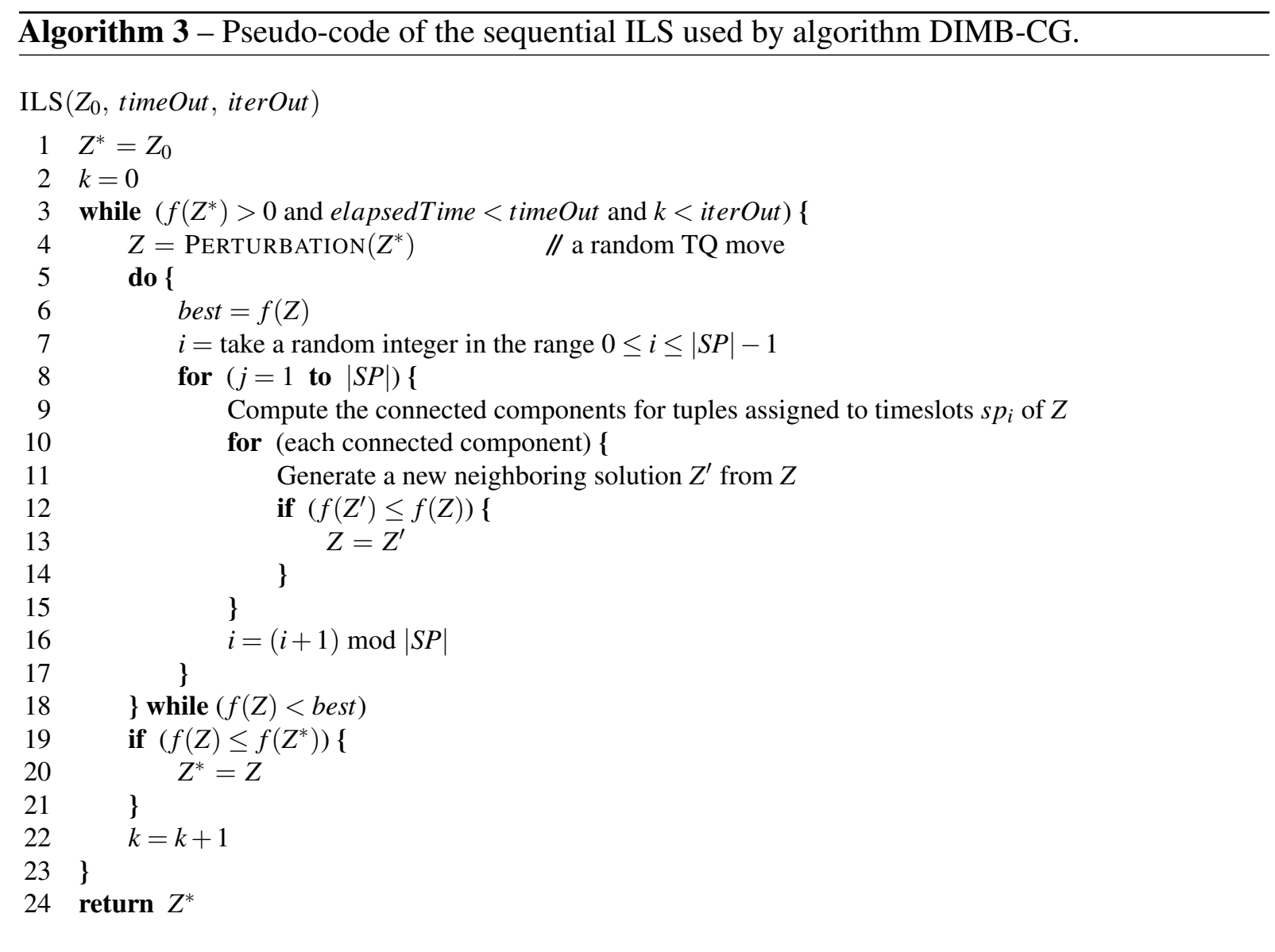

Timeslots

\begin{tabular}{|c|c|c|c|c|c|c|}
\hline & $s_{0}$ & $s_{1}$ & $s_{2}$ & $s_{3}$ & $s_{4}$ & \\
\hline subproblem of teacher $t_{0}$ & 1 & 2 & & 1 & 2 & solved \\
\hline subproblem of teacher $t_{1}$ & 2 & 1 & 3 & 3 & & solved \\
\hline subproblem of teacher $t_{2}$ & 4 & & 0 & 2 & 3 & solved \\
\hline subproblem of teacher $t_{3}$ & & 3 & 4 & 4 & 0 & solved \\
\hline subproblem of teacher $t_{4}$ & & & & & & unsolved $(0,0,2,4)$ \\
\hline subproblem of teacher $t_{5}$ & & & & & & unsolved $(0,1,1,3,4)$ \\
\hline
\end{tabular}

Figure 17 - Preprocessing of variables belonging to class $c_{2}$ in subproblem $t_{4}$.

Minimize $\sum_{x_{r d h} \in J_{0}} x_{r d h}+\sum_{x_{r d h} \in J_{1}}\left(1-x_{r d h}\right)$

Subject to:

Constraints of formulation $C F 1$

$x_{r d h} \in\{0,1\} \quad \forall r \in R ; d \in D ; h \in H$

The linear relaxation of a proximity problem is usually very tight (FISCHETTI; MONACI, 
2014). In our case, model CF1 worked better than model CF2 to formulate the proximity problem. Program (5.1) - (5.2) can be quickly solved to optimality, which makes it an effective tool to recover the feasibility of a solution under construction.

\section{Diversifier}

The diversifier procedure is shown in Algorithm 4. This procedure is responsible for feeding the diversification memory with new solutions that are constructed by exploiting the pool of columns. At each iteration of the main loop (lines 2 to 17), the algorithm constructs a solution $X$ in line 8 (possibly infeasible), that is repaired in line 9 , converted to a timetable $Z$ in line 10 , further improved in line 11 , and sent to the diversification memory.

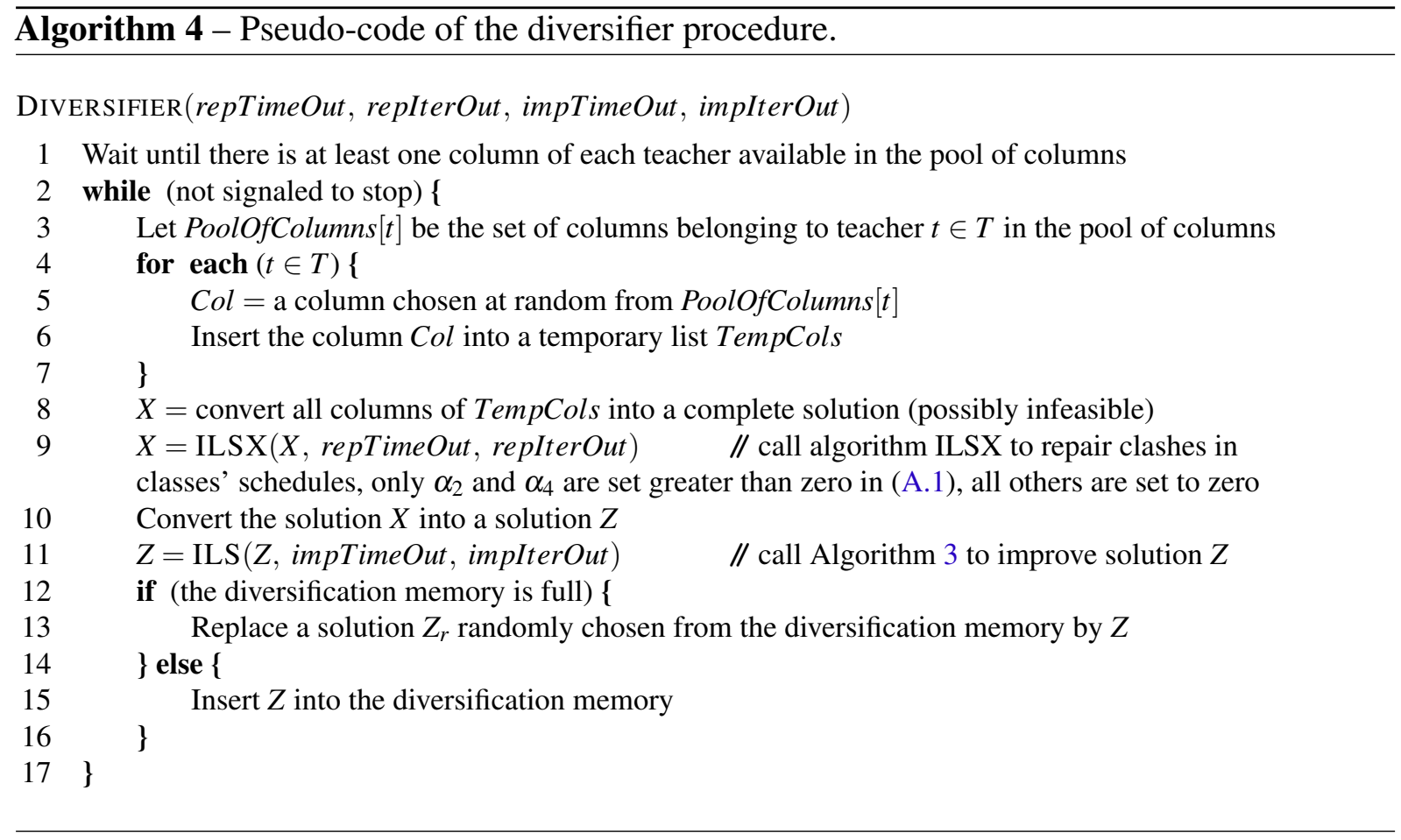

Note that we use the timetable encoding type $X$ to repair solutions, and the timetable encoding type $Z$ to improve solutions. The reason why we do not use the timetable encoding $X$ in both phases is that it is computationally less efficient than the timetable encoding $Z$. The improvement algorithm ILSX (line 9) is an extension of the Algorithm 3 to manage timetables of type $X$. Its extension from Algorithm 3 is simple and straightforward.

\section{Manager}

The manager procedure is shown in Algorithm 5. This procedure is responsible for implementing the policy to coordinate the search performed by the intensifier agents. It first sends one solution from the diversification memory to each intensifier, through the input buffer (lines 4 to 7), and then it enters into a loop (lines 8 to 27). At each iteration of the loop, it pulls one solution from the output buffer, returned by the intensifiers (line 10), decides if the solution 
is accepted in the intensification memory or discarded (lines 12 to 14), and then it puts another solution into the input buffer (lines 18 to 25). In this last statements, the solution is chosen from the diversification memory with probability $\rho$ and from the intensification memory with probability $(1-\rho)$.

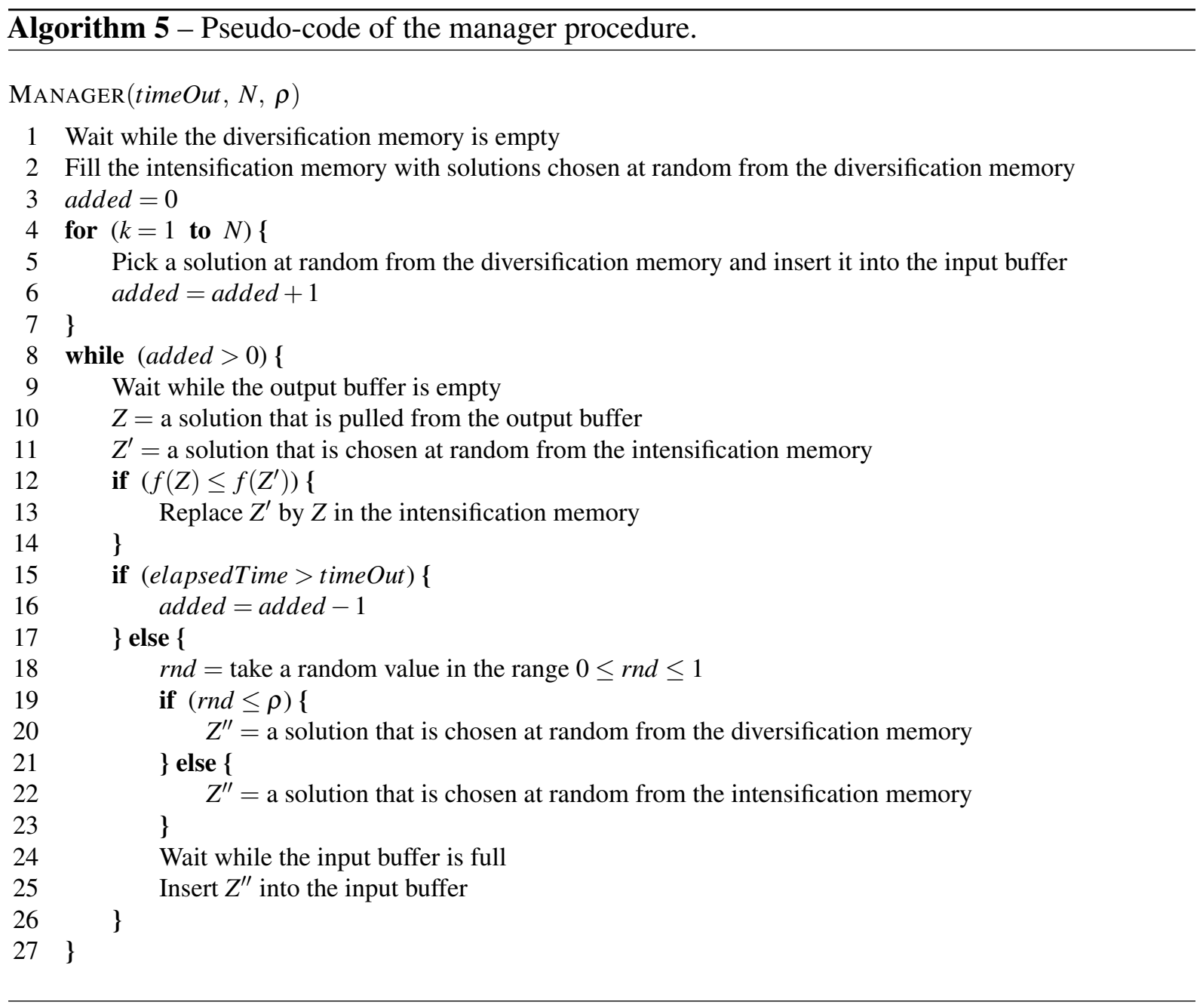

\section{Intensifiers}

The pseudo-code of an intensifier procedure is shown in Algorithm 6. The main idea is that a team of $N$ intensifier metaheuristics run concurrently, cooperating with each other via exchanging of best solutions, to intensify the search around the solutions received from the manager. Each intensifier agent is an ILS (Algorithm 3) that pulls a solution from the input buffer (line 3), explores its neighborhood during a parameter-defined amount of time (line 4), and then returns its best solution to the output buffer (line 6).

Table 26 describes the complete list of parameter values that we used in the implementation of our algorithm DIMB-CG. 


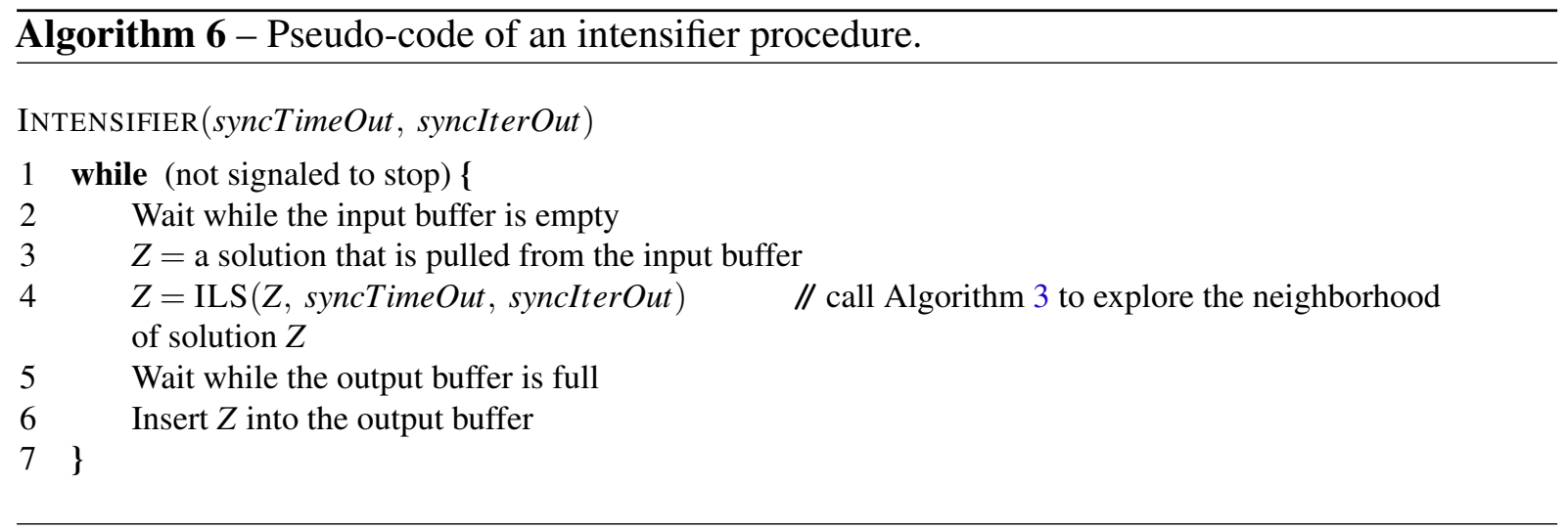

Table 26 - List of parameter values used in the algorithm DIMB-CG.

\begin{tabular}{|c|c|c|}
\hline Component & Parameter & Value \\
\hline Diversification memory & Size & 8 \\
\hline Intensification memory & Size & 1 \\
\hline Input buffer & Size & 19 \\
\hline Output buffer & Size & 19 \\
\hline Buffer of dual values & Size & 3 \\
\hline Buffer of columns & Size & $2 \cdot|T|$ \\
\hline \multirow[t]{3}{*}{ CCGH heuristic } & Proximity program time limit & 30 seconds \\
\hline & ILS time limit (impTimeOut) & 10 seconds \\
\hline & ILS iterations (impIterOut) & 3 iterations \\
\hline \multirow[t]{4}{*}{ Diversifier } & ILSX time limit (repTimeOut) & 1000 iterations \\
\hline & ILSX iterations (repIterOut) & 100 seconds \\
\hline & ILS time limit (impTimeOut) & 10 seconds \\
\hline & ILS iterations (impIterOut) & 3 iterations \\
\hline \multirow[t]{3}{*}{ Manager } & Time limit (timeOut) & 625 seconds \\
\hline & Number of intensifiers $(N)$ & 19 \\
\hline & Diversification probability $(\rho)$ & 0.05 \\
\hline \multirow[t]{2}{*}{ Intensifiers } & Synchronization time (syncTimeOut) & 2 seconds \\
\hline & Synchronization iterations (syncIterOut) & 10000000 iterations \\
\hline
\end{tabular}

\subsection{Computational experiments}

In this section, we report two experiment sets to assess our parallel algorithm DIMB-CG.

Section 5.2.1: we test the effect of inserting the CCGH extra columns into the master program of the CG procedure. We also analyze the behavior of the two strategies used by our constructive heuristic CCGH and the quality of its solutions.

Section 5.2.2: we compare the algorithm DIMB-CG with the best parallel algorithm of Chapter 4.

For problems HSTP-A and HSTP-C, we report experiments with instances of Table 15, and for problem HSTP-B, we use the instances of Table 20.

The proposed algorithm was coded in C++ and compiled with GNU Compiler Collection 4.4.7. The experiments were performed on a server with two cores Intel Xeon E5-2680v2 (2.8 GHz) and $128 \mathrm{~GB}$ of RAM, running Red Hat Enterprise Linux 6.5. In all experiments, 
the algorithm DIMB-CG was allowed to execute during a time limit of 625 seconds, and 25 independent trials were carried out for each instance.

\subsubsection{Analysis of the CCGH heuristic}

We start this section by analyzing the speed-up obtained by the CG procedure with the addition of extra columns into its master program.

Table 27 - Comparison of convergence time (in seconds) between SCG and CG+CCGH.

\begin{tabular}{|c|c|c|c|c|c|c|c|c|c|}
\hline \multirow[b]{2}{*}{ ID } & \multicolumn{3}{|c|}{ HSTP-A } & \multicolumn{3}{|c|}{ HSTP-B } & \multicolumn{3}{|c|}{ HSTP-C } \\
\hline & SCG & $\mathrm{CG}+\mathrm{CCGH}$ & $\mathrm{Sp}$ & SCG & $\mathrm{CG}+\mathrm{CCGH}$ & Sp & SCG & $\mathrm{CG}+\mathrm{CCGH}$ & Sp \\
\hline 1 & 47.16 & 30.92 & 1.53 & 14.66 & 8.93 & 1.64 & 49.06 & 51.49 & 0.95 \\
\hline 2 & 47.89 & 30.81 & 1.55 & 24.60 & 10.20 & 2.41 & 49.83 & 50.72 & 0.98 \\
\hline 3 & 62.40 & 34.00 & 1.84 & 22.54 & 12.86 & 1.75 & 58.25 & 36.94 & 1.58 \\
\hline 4 & 64.63 & 31.70 & 2.04 & 35.08 & 19.33 & 1.81 & 59.99 & 35.29 & 1.70 \\
\hline 5 & 35.82 & 19.42 & 1.84 & 39.73 & 16.19 & 2.45 & 32.32 & 20.84 & 1.55 \\
\hline 6 & 75.69 & 41.71 & 1.81 & 89.80 & 26.68 & 3.37 & 71.08 & 45.25 & 1.57 \\
\hline 7 & 118.60 & 89.52 & 1.32 & 82.39 & 31.73 & 2.60 & 95.15 & 99.07 & 0.96 \\
\hline 8 & 22.19 & 20.28 & 1.09 & & & & 21.86 & 26.18 & 0.83 \\
\hline 9 & 92.47 & 74.04 & 1.25 & & & & 59.83 & 65.53 & 0.91 \\
\hline 10 & 19.66 & 11.11 & 1.77 & & & & 18.17 & 10.13 & 1.79 \\
\hline 11 & 108.70 & 82.54 & 1.32 & & & & 76.99 & 83.06 & 0.93 \\
\hline 12 & 88.33 & 72.73 & 1.21 & & & & 82.87 & 81.83 & 1.01 \\
\hline 13 & 3.38 & 3.52 & 0.96 & & & & 2.51 & 2.74 & 0.92 \\
\hline 14 & 71.02 & 48.58 & 1.46 & & & & 48.35 & 49.57 & 0.98 \\
\hline 15 & 8.13 & 6.43 & 1.26 & & & & \multicolumn{3}{|c|}{ Infeasible } \\
\hline 16 & 27.70 & 16.56 & 1.67 & & & & 22.55 & 17.26 & 1.31 \\
\hline 17 & 21.11 & 14.19 & 1.49 & & & & 20.56 & 14.42 & 1.43 \\
\hline 18 & 19.87 & 11.81 & 1.68 & & & & 19.62 & 11.50 & 1.71 \\
\hline 19 & 13.01 & 7.53 & 1.73 & & & & 15.60 & 8.08 & 1.93 \\
\hline 20 & 20.35 & 9.69 & 2.10 & & & & 24.83 & 12.09 & 2.05 \\
\hline 21 & 97.66 & 69.72 & 1.40 & & & & 73.70 & 74.36 & 0.99 \\
\hline 22 & 51.53 & 36.89 & 1.40 & & & & \multicolumn{3}{|c|}{ Infeasible } \\
\hline 23 & 302.46 & 189.38 & 1.60 & & & & 273.51 & 280.97 & 0.97 \\
\hline 24 & 208.42 & 149.69 & 1.39 & & & & 201.99 & 204.41 & 0.99 \\
\hline 25 & 72.36 & 52.94 & 1.37 & & & & 56.54 & 61.29 & 0.92 \\
\hline 26 & 49.56 & 31.78 & 1.56 & & & & 37.02 & 33.03 & 1.12 \\
\hline 27 & 37.01 & 25.95 & 1.43 & & & & 27.67 & 29.22 & 0.95 \\
\hline 28 & 119.56 & 70.20 & 1.70 & & & & 95.61 & 77.83 & 1.23 \\
\hline 29 & 106.92 & 59.89 & 1.79 & & & & 115.67 & 89.65 & 1.29 \\
\hline 30 & 126.22 & 68.76 & 1.84 & & & & 107.54 & 86.26 & 1.25 \\
\hline 31 & 94.32 & 63.15 & 1.49 & & & & 79.59 & 68.76 & 1.16 \\
\hline 32 & 72.45 & 47.77 & 1.52 & & & & 78.22 & 67.08 & 1.17 \\
\hline 33 & 75.38 & 50.19 & 1.50 & & & & 67.91 & 56.06 & 1.21 \\
\hline 34 & 61.65 & 40.10 & 1.54 & & & & 73.85 & 74.17 & 1.00 \\
\hline Avg & & & 1.54 & & & 2.29 & & & 1.23 \\
\hline
\end{tabular}

Table 27 shows the convergence time of the stand-alone CG algorithm (SCG) compared to the time required when the $\mathrm{CCGH}$ extra columns are also inserted into the master program (CG+CCGH). This table shows that the column generation process is accelerated when the extra columns are also added to the master program, and the column (Sp) is the median speed-up obtained. We observe that for problems HSTP-A and HSTP-B, the use of the constructive 
heuristic CCGH introduced reasonable speed-ups on average. However, for problem HSTP-C, it was less efficient. Given that problem HSTP-C is more constrained than the other problems, we have observed that the proximity program takes longer to be solved. Therefore, very few extra columns are generated, which are insufficient to provide reasonable speed-ups.

Table 28 presents results regarding the number of columns necessary to converge the CG algorithm. Column (PoAC) is the percentage of additional columns generated when the CCGH heuristic is employed. This table shows that for problems HSTP-A and HSTP-C, the master program required, on average, more columns to converge when using our $\mathrm{CG}+\mathrm{CCGH}$ strategy, than when using the stand-alone column generation. However, for problem HSTP-B, the master program required fewer columns when using the $\mathrm{CG}+\mathrm{CCGH}$ strategy.

Table 28 - Number of columns generated by the SCG compared to CG+CCGH.

\begin{tabular}{|c|c|c|c|c|c|c|c|c|c|}
\hline \multirow[b]{2}{*}{ ID } & \multicolumn{3}{|c|}{ HSTP-A } & \multicolumn{3}{|c|}{ HSTP-B } & \multicolumn{3}{|c|}{ HSTP-C } \\
\hline & SCG & $\mathrm{CG}+\mathrm{CCGH}$ & PoAC & SCG & $\mathrm{CG}+\mathrm{CCGH}$ & PoAC & SCG & $\mathrm{CG}+\mathrm{CCGH}$ & PoAC \\
\hline 1 & 1782 & 1885 & 5.78 & 371 & 355 & -4.31 & 1828 & 2069 & 13.18 \\
\hline 2 & 1770 & 1863 & 5.25 & 848 & 640 & -24.53 & 1817 & 2005 & 10.35 \\
\hline 3 & 2032 & 1795 & -11.66 & 904 & 823 & -8.96 & 2114 & 2106 & -0.38 \\
\hline 4 & 2065 & 1733 & -16.08 & 1364 & 1156 & -15.25 & 2131 & 2033 & -4.60 \\
\hline 5 & 1412 & 1266 & -10.34 & 1677 & 1238 & -26.18 & 1427 & 1539 & 7.85 \\
\hline 6 & 2183 & 2046 & -6.28 & 2025 & 1338 & -33.93 & 2282 & 2280 & -0.09 \\
\hline 7 & 3517 & 4685 & 33.21 & 2216 & 1588 & -28.34 & 3281 & 3470 & 5.76 \\
\hline 8 & 1266 & 2094 & 65.40 & & & & 1293 & 1388 & 7.35 \\
\hline 9 & 2703 & 4255 & 57.42 & & & & 2200 & 2315 & 5.23 \\
\hline 10 & 891 & 845 & -5.16 & & & & 884 & 857 & -3.05 \\
\hline 11 & 3191 & 4194 & 31.43 & & & & 2908 & 2967 & 2.03 \\
\hline 12 & 2971 & 3198 & 7.64 & & & & 2946 & 2998 & 1.77 \\
\hline 13 & 347 & 568 & 63.69 & & & & 302 & 399 & 32.12 \\
\hline 14 & 2767 & 2951 & 6.65 & & & & 2476 & 2525 & 1.98 \\
\hline 15 & 589 & 710 & 20.54 & & & & \multicolumn{3}{|c|}{ Infeasible } \\
\hline 16 & 1195 & 1147 & -4.02 & & & & 1196 & 1285 & 7.44 \\
\hline 17 & 999 & 1059 & 6.01 & & & & 1078 & 1226 & 13.73 \\
\hline 18 & 869 & 870 & 0.12 & & & & 859 & 893 & 3.96 \\
\hline 19 & 673 & 661 & -1.78 & & & & 738 & 667 & -9.62 \\
\hline 20 & 933 & 784 & -15.97 & & & & 1034 & 923 & -10.74 \\
\hline 21 & 2933 & 3027 & 3.20 & & & & 2760 & 2767 & 0.25 \\
\hline 22 & 1915 & 2534 & 32.32 & & & & \multicolumn{3}{|c|}{ Infeasible } \\
\hline 23 & 6096 & 7061 & 15.83 & & & & 6107 & 6547 & 7.20 \\
\hline 24 & 5741 & 7439 & 29.58 & & & & 5832 & 6206 & 6.41 \\
\hline 25 & 2546 & 2954 & 16.03 & & & & 2408 & 2552 & 5.98 \\
\hline 26 & 1686 & 1887 & 11.92 & & & & 1594 & 1726 & 8.28 \\
\hline 27 & 1409 & 1664 & 18.10 & & & & 1322 & 1431 & 8.25 \\
\hline 28 & 3282 & 3569 & 8.74 & & & & 3067 & 3679 & 19.95 \\
\hline 29 & 3128 & 3211 & 2.65 & & & & 3136 & 3908 & 24.62 \\
\hline 30 & 3277 & 3428 & 4.61 & & & & 3154 & 3859 & 22.35 \\
\hline 31 & 3075 & 3594 & 16.88 & & & & 2966 & 3190 & 7.55 \\
\hline 32 & 2601 & 2794 & 7.42 & & & & 2658 & 3459 & 30.14 \\
\hline 33 & 2696 & 2820 & 4.60 & & & & 2613 & 3183 & 21.81 \\
\hline 34 & 2472 & 2623 & 6.11 & & & & 2557 & 3573 & 39.73 \\
\hline \multicolumn{2}{|c|}{ Avg.: } & & 12.05 & & & -20.21 & & & 8.96 \\
\hline
\end{tabular}

In Table 29, we analyze the effectiveness of the two strategies used in our CCGH heuristic. Column (PoS1) is the percentage of success in constructing feasible solutions using 
the first strategy, which solves each subproblem by fixing some of its variables. Column (PoSS) is the percentage of solved subproblems before reaching infeasibility. Column (PoS2) is the percentage of success in finding feasible solutions using the second strategy, which solves the proximity problem. This table shows that the fixing of variables is ineffective to construct complete solutions. However, it defines most of the teachers' schedules without clashes (more than $85.8 \%$ on average). This fact may explain the high rate of success in which the proximity program succeeded in finding feasible solutions, more than $99.5 \%$ of the trials on average.

Table 29 - Effectiveness of the two strategies used by the CCGH heuristic.

\begin{tabular}{|c|c|c|c|c|c|c|c|c|c|}
\hline \multirow[b]{2}{*}{ ID } & \multicolumn{3}{|c|}{ HSTP-A } & \multicolumn{3}{|c|}{ HSTP-B } & \multicolumn{3}{|c|}{ HSTP-C } \\
\hline & PoS1 & PoSS & PoS2 & PoS1 & PoSS & PoS2 & PoS1 & PoSS & PoS2 \\
\hline 1 & 0.0 & 87.4 & 100.0 & 2.7 & 86.7 & 100.0 & 0.0 & 87.7 & 100.0 \\
\hline 2 & 0.0 & 87.6 & 100.0 & 7.4 & 92.5 & 100.0 & 0.0 & 87.8 & 100.0 \\
\hline 3 & 0.3 & 93.5 & 100.0 & 0.0 & 86.1 & 100.0 & 0.3 & 93.6 & 100.0 \\
\hline 4 & 1.4 & 94.1 & 100.0 & 0.0 & 84.4 & 100.0 & 1.6 & 94.3 & 100.0 \\
\hline 5 & 3.3 & 94.0 & 100.0 & 0.3 & 94.2 & 100.0 & 4.1 & 94.2 & 100.0 \\
\hline 6 & 0.0 & 91.9 & 100.0 & 0.3 & 92.9 & 100.0 & 0.1 & 91.9 & 100.0 \\
\hline 7 & 0.0 & 78.0 & 100.0 & 0.0 & 91.4 & 100.0 & 0.0 & 77.7 & 100.0 \\
\hline 8 & 0.0 & 77.6 & 100.0 & & & & 0.0 & 78.1 & 100.0 \\
\hline 9 & 0.0 & 76.7 & 100.0 & & & & 0.0 & 74.7 & 100.0 \\
\hline 10 & 7.5 & 94.2 & 100.0 & & & & 6.7 & 94.2 & 100.0 \\
\hline 11 & 0.0 & 81.5 & 100.0 & & & & 0.0 & 80.5 & 100.0 \\
\hline 12 & 0.0 & 76.1 & 100.0 & & & & 0.0 & 76.5 & 99.4 \\
\hline 13 & 0.6 & 80.8 & 100.0 & & & & 0.4 & 79.0 & 100.0 \\
\hline 14 & 0.0 & 77.3 & 100.0 & & & & 0.0 & 75.2 & 100.0 \\
\hline 15 & 0.0 & 81.1 & 100.0 & & & & \multicolumn{3}{|c|}{ Infeasible } \\
\hline 16 & 0.1 & 86.7 & 100.0 & & & & 0.0 & 86.6 & 100.0 \\
\hline 17 & 0.7 & 89.9 & 100.0 & & & & 0.6 & 89.6 & 100.0 \\
\hline 18 & 14.0 & 93.9 & 100.0 & & & & 11.3 & 93.7 & 100.0 \\
\hline 19 & 21.3 & 94.7 & 100.0 & & & & 18.5 & 94.5 & 100.0 \\
\hline 20 & 8.3 & 94.1 & 100.0 & & & & 4.7 & 93.6 & 100.0 \\
\hline 21 & 0.0 & 78.5 & 100.0 & & & & 0.0 & 77.2 & 85.2 \\
\hline 22 & 0.0 & 78.6 & 100.0 & & & & \multicolumn{3}{|c|}{ Infeasible } \\
\hline 23 & 0.0 & 81.5 & 100.0 & & & & 0.0 & 81.2 & 100.0 \\
\hline 24 & 0.0 & 79.4 & 100.0 & & & & 0.0 & 79.0 & 100.0 \\
\hline 25 & 0.0 & 82.6 & 100.0 & & & & 0.0 & 81.9 & 100.0 \\
\hline 26 & 0.0 & 81.7 & 100.0 & & & & 0.0 & 82.5 & 100.0 \\
\hline 27 & 0.0 & 80.1 & 100.0 & & & & 0.0 & 79.6 & 100.0 \\
\hline 28 & 0.0 & 89.5 & 100.0 & & & & 0.0 & 89.2 & 100.0 \\
\hline 29 & 0.0 & 89.0 & 100.0 & & & & 0.0 & 88.7 & 100.0 \\
\hline 30 & 0.0 & 89.2 & 100.0 & & & & 0.0 & 89.0 & 100.0 \\
\hline 31 & 0.0 & 87.0 & 100.0 & & & & 0.0 & 86.6 & 100.0 \\
\hline 32 & 0.0 & 89.7 & 100.0 & & & & 0.0 & 89.7 & 100.0 \\
\hline 33 & 0.0 & 89.6 & 100.0 & & & & 0.0 & 89.3 & 100.0 \\
\hline 34 & 0.0 & 88.2 & 100.0 & & & & 0.0 & 88.1 & 100.0 \\
\hline Avg.: & 1.7 & 85.8 & 100.0 & 1.5 & 89.7 & 100.0 & 1.5 & 85.8 & 99.5 \\
\hline
\end{tabular}

In Table 30, we analyze the quality of the solutions constructed by the CCGH heuristic. This table shows that the proposed heuristic is very effective indeed. It builds feasible solutions with an average optimality gap of $19.17 \%$ for problem HSTP-A and $10.57 \%$ for problem HSTP-C. For problem HSTP-B, the produced solutions are almost optimal, with an average gap of only $2.74 \%$. 
Table 30 - Quality of the CCGH solutions.

\begin{tabular}{|c|c|c|c|c|c|c|c|c|c|}
\hline \multirow[b]{2}{*}{ ID } & \multicolumn{3}{|c|}{ HSTP-A } & \multicolumn{3}{|c|}{ HSTP-B } & \multicolumn{3}{|c|}{ HSTP-C } \\
\hline & Best LB & Mean & Gap & Best LB & Mean & Gap & Best LB & Mean & Gap \\
\hline 1 & 950 & 1170 & 18.80 & 202 & 209 & 3.35 & 699 & 787 & 11.18 \\
\hline 2 & 950 & 1170 & 18.80 & 333 & 342 & 2.63 & 699 & 786 & 11.07 \\
\hline 3 & 1010 & 1210 & 16.53 & 423 & 462 & 8.44 & 742 & 836 & 11.24 \\
\hline 4 & 990 & 1200 & 17.50 & 652 & 656 & 0.61 & 734 & 832 & 11.78 \\
\hline 5 & 870 & 970 & 10.31 & 762 & 772 & 1.30 & 631 & 682 & 7.48 \\
\hline 6 & 1080 & 1320 & 18.18 & 756 & 768 & 1.56 & 772 & 864 & 10.65 \\
\hline 7 & 1690 & 2310 & 26.84 & 1017 & 1030 & 1.26 & 1212 & 1372 & 11.66 \\
\hline 8 & 950 & 1285 & 26.07 & & & & 675 & 735 & 8.16 \\
\hline 9 & 1100 & 1370 & 19.71 & & & & 807 & 890 & 9.33 \\
\hline 10 & 420 & 500 & 16.00 & & & & 298 & 331 & 9.97 \\
\hline 11 & 1400 & 1880 & 25.53 & & & & 961 & 1124 & 14.50 \\
\hline 12 & 1450 & 2120 & 31.60 & & & & 1018 & 1104 & 7.79 \\
\hline 13 & 420 & 460 & 8.70 & & & & 273 & 273 & 0.00 \\
\hline 14 & 1270 & 1755 & 27.64 & & & & 933 & 1016 & 8.17 \\
\hline 15 & 395 & 465 & 15.05 & & & & \multicolumn{3}{|c|}{ Infeasible } \\
\hline 16 & 640 & 760 & 15.79 & & & & 481 & 547 & 12.07 \\
\hline 17 & 580 & 700 & 17.14 & & & & 457 & 512 & 10.74 \\
\hline 18 & 460 & 530 & 13.21 & & & & 319 & 360 & 11.39 \\
\hline 19 & 360 & 410 & 12.20 & & & & 254 & 277 & 8.30 \\
\hline 20 & 470 & 540 & 12.96 & & & & 325 & 362 & 10.22 \\
\hline 21 & 1450 & 1990 & 27.14 & & & & 1050 & 1148 & 8.54 \\
\hline 22 & 1035 & 1350 & 23.33 & & & & \multicolumn{3}{|c|}{ Infeasible } \\
\hline 23 & 2505 & 3350 & 25.22 & & & & 1813 & 2069 & 12.37 \\
\hline 24 & 2680 & 3625 & 26.07 & & & & 1988 & 2256 & 11.88 \\
\hline 25 & 1315 & 1775 & 25.92 & & & & 912 & 1057 & 13.72 \\
\hline 26 & 750 & 940 & 20.21 & & & & 570 & 640 & 10.94 \\
\hline 27 & 730 & 910 & 19.78 & & & & 552 & 591 & 6.60 \\
\hline 28 & 1570 & 1850 & 15.14 & & & & 1124 & 1271 & 11.57 \\
\hline 29 & 1570 & 1870 & 16.04 & & & & 1116 & 1267 & 11.92 \\
\hline 30 & 1590 & 1890 & 15.87 & & & & 1133 & 1287 & 11.97 \\
\hline 31 & 1570 & 1910 & 17.80 & & & & 1127 & 1299 & 13.24 \\
\hline 32 & 1390 & 1670 & 16.77 & & & & 1014 & 1170 & 13.33 \\
\hline 33 & 1410 & 1680 & 16.07 & & & & 1018 & 1170 & 12.99 \\
\hline 34 & 1390 & 1690 & 17.75 & & & & 1005 & 1163 & 13.59 \\
\hline $\mathrm{Avg}$ & & & 19.17 & & & 2.74 & & & 10.57 \\
\hline
\end{tabular}

\subsubsection{Analysis of the DIMB-CG algorithm}

In this section, we compare the proposed algorithm DIMB-CG with the parallel algorithm DIMB-19I1D-8-0.05 of Chapter 4.

In Table 31, we present the results for problem HSTP-A. Column (Best LB) shows the best lower bounds reported in Section 3.3. The best solutions are shown in bold font. This table shows that our algorithm performs better than DIMB-19I1D-8-0.05, both in terms of median and best solutions. In column (ToBS), we show the median computational time of the best solution found by algorithm DIMB-CG, which is the time when the algorithm made the last improvement in its global best solution. This column shows that, in the median case, the algorithm takes less than 500 seconds to find near-optimal solutions, even for the largest instances 23 and 24. Also, for some small instances, such as 10,15 and 18 to 20 , the algorithm only takes a few seconds to 
find the optimal solutions.

Table 31 - Results of the algorithm DIMB-CG compared to DIMB-19I1D-8-0.05 on problem HSTP-A.

\begin{tabular}{|c|c|c|c|c|c|c|c|c|c|c|}
\hline \multirow[b]{2}{*}{ ID } & \multirow[b]{2}{*}{ Best LB } & \multicolumn{5}{|c|}{ DIMB-CG } & \multicolumn{4}{|c|}{ DIMB-19I1D-8-0.05 } \\
\hline & & ToBS (s) & Median & Gap & Best & Gap & Median & Gap & Best & Gap \\
\hline 1 & 950 & 306.90 & 960 & 1.04 & 950 & 0.00 & 970 & 2.06 & 950 & 0.00 \\
\hline 2 & 950 & 349.62 & 960 & 1.04 & 950 & 0.00 & 970 & 2.06 & 950 & 0.00 \\
\hline 3 & 1010 & 204.64 & 1010 & 0.00 & 1010 & 0.00 & 1020 & 0.98 & 1010 & 0.00 \\
\hline 4 & 990 & 148.24 & 990 & 0.00 & 990 & 0.00 & 1000 & 1.00 & 990 & 0.00 \\
\hline 5 & 870 & 29.34 & 870 & 0.00 & 870 & 0.00 & 870 & 0.00 & 870 & 0.00 \\
\hline 6 & 1080 & 220.62 & 1080 & 0.00 & 1080 & 0.00 & 1100 & 1.82 & 1080 & 0.00 \\
\hline 7 & 1690 & 455.17 & 1830 & 7.65 & 1770 & 4.52 & 1880 & 10.11 & 1810 & 6.63 \\
\hline 8 & 950 & 38.78 & 1045 & 9.09 & 980 & 3.06 & 1030 & 7.77 & 980 & 3.06 \\
\hline 9 & 1100 & 269.50 & 1160 & 5.17 & 1130 & 2.65 & 1170 & 5.98 & 1130 & 2.65 \\
\hline 10 & 420 & 8.42 & 420 & 0.00 & 420 & 0.00 & 420 & 0.00 & 420 & 0.00 \\
\hline 11 & 1400 & 378.52 & 1520 & 7.89 & 1470 & 4.76 & 1530 & 8.50 & 1450 & 3.45 \\
\hline 12 & 1450 & 332.04 & 1580 & 8.23 & 1500 & 3.33 & 1600 & 9.38 & 1530 & 5.23 \\
\hline 13 & 420 & 46.25 & 420 & 0.00 & 420 & 0.00 & 420 & 0.00 & 420 & 0.00 \\
\hline 14 & 1270 & 313.59 & 1340 & 5.22 & 1290 & 1.55 & 1360 & 6.62 & 1300 & 2.31 \\
\hline 15 & 395 & 11.92 & 395 & 0.00 & 395 & 0.00 & 395 & 0.00 & 395 & 0.00 \\
\hline 16 & 640 & 77.04 & 640 & 0.00 & 640 & 0.00 & 650 & 1.54 & 640 & 0.00 \\
\hline 17 & 580 & 147.30 & 580 & 0.00 & 580 & 0.00 & 590 & 1.69 & 580 & 0.00 \\
\hline 18 & 460 & 6.45 & 460 & 0.00 & 460 & 0.00 & 460 & 0.00 & 460 & 0.00 \\
\hline 19 & 360 & 4.29 & 360 & 0.00 & 360 & 0.00 & 360 & 0.00 & 360 & 0.00 \\
\hline 20 & 470 & 8.45 & 470 & 0.00 & 470 & 0.00 & 470 & 0.00 & 470 & 0.00 \\
\hline 21 & 1450 & 432.95 & 1520 & 4.61 & 1480 & 2.03 & 1550 & 6.45 & 1480 & 2.03 \\
\hline 22 & 1035 & 251.71 & 1075 & 3.72 & 1045 & 0.96 & 1095 & 5.48 & 1055 & 1.90 \\
\hline 23 & 2505 & 490.41 & 2740 & 8.58 & 2670 & 6.18 & 2790 & 10.22 & 2670 & 6.18 \\
\hline 24 & 2680 & 477.62 & 2920 & 8.22 & 2840 & 5.63 & 2970 & 9.76 & 2860 & 6.29 \\
\hline 25 & 1315 & 401.50 & 1380 & 4.71 & 1340 & 1.87 & 1400 & 6.07 & 1340 & 1.87 \\
\hline 26 & 750 & 284.05 & 770 & 2.60 & 750 & 0.00 & 780 & 3.85 & 750 & 0.00 \\
\hline 27 & 730 & 249.81 & 740 & 1.35 & 730 & 0.00 & 750 & 2.67 & 730 & 0.00 \\
\hline 28 & 1570 & 319.46 & 1600 & 1.88 & 1590 & 1.26 & 1620 & 3.09 & 1590 & 1.26 \\
\hline 29 & 1570 & 444.11 & 1600 & 1.88 & 1580 & 0.63 & 1620 & 3.09 & 1590 & 1.26 \\
\hline 30 & 1590 & 343.04 & 1620 & 1.85 & 1610 & 1.24 & 1640 & 3.05 & 1600 & 0.63 \\
\hline 31 & 1570 & 421.71 & 1620 & 3.09 & 1600 & 1.88 & 1640 & 4.27 & 1590 & 1.26 \\
\hline 32 & 1390 & 437.21 & 1420 & 2.11 & 1410 & 1.42 & 1440 & 3.47 & 1410 & 1.42 \\
\hline 33 & 1410 & 307.89 & 1450 & 2.76 & 1440 & 2.08 & 1460 & 3.42 & 1430 & 1.40 \\
\hline 34 & 1390 & 427.91 & 1430 & 2.80 & 1420 & 2.11 & 1450 & 4.14 & 1410 & 1.42 \\
\hline $\mathrm{Ra}$ & & & 33 & & 29 & & 8 & & 28 & \\
\hline$A v$ & & & & 2.81 & & 1.39 & & 3.78 & & 1.48 \\
\hline
\end{tabular}

In Table 32, we present the results for problem HSTP-B. This table shows that both algorithms were able to find the optimal solutions for all instances in the median case. Column (ToBS) shows that the algorithm DIMB-CG is almost instantaneous to find optimal solutions for these instances, except for instance 3, in which it takes around 30.55 seconds to reach the optimal solutions.

Table 33 presents the results for problem HSTP-C. This table shows that the algorithm DIMB-CG performs better than the algorithm DIMB-19I1D-8-0.05 in median solutions, but it is less effective in best solutions. As the problem HSTP-C is more constrained than the problem HSTP-A, the algorithm DIMB-CG takes longer to make the last improvement, see column (ToBS). However, it is still able to reach near-optimal solutions expending running time much 
smaller than the specified time limit for most of the tested instances.

Table 32 - Results of the algorithm DIMB-CG compared to DIMB-19I1D-8-0.05 on problem HSTP-B.

\begin{tabular}{|c|c|c|c|c|c|c|c|c|c|c|}
\hline \multirow[b]{2}{*}{ ID } & \multirow[b]{2}{*}{ Best LB } & \multicolumn{5}{|c|}{ DIMB-CG } & \multicolumn{4}{|c|}{ DIMB-19I1D-8-0.05 } \\
\hline & & ToBS (s) & Median & Gap & Best & Gap & Median & Gap & Best & Gap \\
\hline 1 & 202 & 2.17 & 202 & 0.00 & 202 & 0.00 & 202 & 0.00 & 202 & 0.00 \\
\hline 2 & 333 & 2.44 & 333 & 0.00 & 333 & 0.00 & 333 & 0.00 & 333 & 0.00 \\
\hline 3 & 423 & 30.55 & 423 & 0.00 & 423 & 0.00 & 423 & 0.00 & 423 & 0.00 \\
\hline 4 & 652 & 4.67 & 652 & 0.00 & 652 & 0.00 & 652 & 0.00 & 652 & 0.00 \\
\hline 5 & 762 & 2.94 & 762 & 0.00 & 762 & 0.00 & 762 & 0.00 & 762 & 0.00 \\
\hline 6 & 756 & 3.00 & 756 & 0.00 & 756 & 0.00 & 756 & 0.00 & 756 & 0.00 \\
\hline 7 & 1017 & 3.62 & 1017 & 0.00 & 1017 & 0.00 & 1017 & 0.00 & 1017 & 0.00 \\
\hline $\mathrm{Ra}$ & & & 7 & & 7 & & 7 & & 7 & \\
\hline Av & & & & 0.00 & & 0.00 & & 0.00 & & 0.00 \\
\hline
\end{tabular}

Table 33 - Results of the algorithm DIMB-CG compared to DIMB-19I1D-8-0.05 on problem HSTP-C.

\begin{tabular}{|c|c|c|c|c|c|c|c|c|c|c|}
\hline \multirow[b]{2}{*}{ ID } & \multirow[b]{2}{*}{ Best LB } & \multicolumn{5}{|c|}{ DIMB-CG } & \multicolumn{4}{|c|}{ DIMB-19I1D-8-0.05 } \\
\hline & & ToBS (s) & Median & Gap & Best & Gap & Median & Gap & Best & Gap \\
\hline 1 & 699 & 364.74 & 708 & 1.27 & 701 & 0.29 & 710 & 1.55 & $\overline{700}$ & 0.14 \\
\hline 2 & 699 & 409.47 & 707 & 1.13 & 701 & 0.29 & 711 & 1.69 & 701 & 0.29 \\
\hline 3 & 742 & 389.30 & 747 & 0.67 & 743 & 0.13 & 749 & 0.93 & 743 & 0.13 \\
\hline 4 & 734 & 419.58 & 738 & 0.54 & 735 & 0.14 & 743 & 1.21 & 735 & 0.14 \\
\hline 5 & 631 & 93.26 & 631 & 0.00 & 631 & 0.00 & 631 & 0.00 & 631 & 0.00 \\
\hline 6 & 772 & 403.62 & 772 & 0.00 & 772 & 0.00 & 777 & 0.64 & 772 & 0.00 \\
\hline 7 & 1212 & 495.65 & 1259 & 3.73 & 1237 & 2.02 & 1270 & 4.57 & 1239 & 2.18 \\
\hline 8 & 675 & 58.63 & 697 & 3.16 & 679 & 0.59 & 698 & 3.30 & 686 & 1.60 \\
\hline 9 & 807 & 357.22 & 821 & 1.71 & 812 & 0.62 & 827 & 2.42 & 814 & 0.86 \\
\hline 10 & 298 & 10.42 & 298 & 0.00 & 298 & 0.00 & 298 & 0.00 & 298 & 0.00 \\
\hline 11 & 961 & 467.90 & 998 & 3.71 & 987 & 2.63 & 1005 & 4.38 & 985 & 2.44 \\
\hline 12 & 1018 & 334.13 & 1039 & 2.02 & 1032 & 1.36 & 1044 & 2.49 & 1030 & 1.17 \\
\hline 13 & 73 & 4.11 & 273 & 0.00 & 273 & 0.00 & 273 & 0.00 & 273 & 0.00 \\
\hline 14 & 933 & 440.61 & 946 & 1.37 & 940 & 0.74 & 954 & 2.20 & 939 & 0.64 \\
\hline 15 & \multicolumn{10}{|c|}{ Infeasible } \\
\hline 16 & 481 & 173.54 & 483 & 0.41 & 481 & 0.00 & 484 & 0.62 & 481 & 0.00 \\
\hline 17 & 457 & 309.41 & 460 & 0.65 & 457 & 0.00 & 461 & 0.87 & 457 & 0.00 \\
\hline 18 & 319 & 22.51 & 319 & 0.00 & 319 & 0.00 & 319 & 0.00 & 319 & 0.00 \\
\hline 19 & 254 & 2.32 & 254 & 0.00 & 254 & 0.00 & 254 & 0.00 & 254 & 0.00 \\
\hline 20 & 325 & 18.41 & 325 & 0.00 & 325 & 0.00 & 325 & 0.00 & 325 & 0.00 \\
\hline 21 & 1050 & 452.70 & 1066 & 1.50 & 1059 & 0.85 & 1074 & 2.23 & 1058 & 0.76 \\
\hline 22 & \multicolumn{10}{|c|}{ Infeasible } \\
\hline 23 & 1813 & 534.02 & 1887 & 3.92 & 1874 & 3.26 & 1910 & 5.08 & 1867 & 2.89 \\
\hline 24 & 1988 & 565.76 & 2059 & 3.45 & 2042 & 2.64 & 2079 & 4.38 & 2038 & 2.45 \\
\hline 25 & 912 & 485.79 & 937 & 2.67 & 920 & 0.87 & 944 & 3.39 & 926 & 1.51 \\
\hline 26 & 570 & 219.81 & 575 & 0.87 & 571 & 0.18 & 576 & 1.04 & 570 & 0.00 \\
\hline 27 & 552 & 306.29 & 555 & 0.54 & 552 & 0.00 & 556 & 0.72 & 552 & 0.00 \\
\hline 28 & 1124 & 459.30 & 1142 & 1.58 & 1132 & 0.71 & 1150 & 2.26 & 1127 & 0.27 \\
\hline 29 & 1116 & 511.92 & 1137 & 1.85 & 1130 & 1.24 & 1145 & 2.53 & 1127 & 0.98 \\
\hline 30 & 1133 & 426.27 & 1153 & 1.73 & 1142 & 0.79 & 1164 & 2.66 & 1142 & 0.79 \\
\hline 31 & 1127 & 511.13 & 1152 & 2.17 & 1140 & 1.14 & 1165 & 3.26 & 1137 & 0.88 \\
\hline 32 & 1014 & 506.90 & 1036 & 2.12 & 1026 & 1.17 & 1043 & 2.78 & 1024 & 0.98 \\
\hline 33 & 1018 & 352.11 & 1039 & 2.02 & 1028 & 0.97 & 1047 & 2.77 & 1034 & 1.55 \\
\hline 34 & 1005 & 417.23 & 1032 & 2.62 & 1020 & 1.47 & 1041 & 3.46 & 1015 & 0.99 \\
\hline Ran & & & 32 & & 19 & & 6 & & 27 & \\
\hline Avg & & & & 1.48 & & 0.75 & & 1.98 & & 0.74 \\
\hline
\end{tabular}




\subsection{Final remarks}

In this chapter, we investigated the effect of exploiting column generation into a cooperative parallel metaheuristic framework to generate solutions and lower bounds for high school timetabling problems. We proposed a parallel algorithm in which a column generation procedure computes lower bounds on the optimal solutions, while two constructive heuristics exploit the generated columns to construct initial feasible solutions that are further improved by a team of cooperative metaheuristics.

As a contribution, we developed new strategies based on mixed-integer programming and metaheuristic techniques, which exploit the columns of the column generation algorithm to create feasible solutions. The computational experiments demonstrated that these strategies are quite effective in constructing good quality feasible solutions. When such heuristic strategies are embedded into the parallel DIMB framework of Chapter 4, the computational experiments suggest that the resulting algorithm performs better than the best algorithm of Chapter 4 . As an overall result, the new algorithm was able to find near-optimal solutions for all three studied problems by expending relatively short execution time.

In this study, we have investigated the construction of solutions by i) converting a subset of columns into a non-clash-free solution that is repaired by a metaheuristic, and ii) solving subproblems by fixing some of their variables based on past solved subproblems. Further research can be done to investigate new constructive strategies to exploit the column generation subproblems. 

CHAPTER

6

$+2$

\section{CONCLUSIONS}

In this thesis, we addressed the high school timetabling problem, which consists in assigning meetings between classes and teachers, with the goal of minimizing the violation of specific soft requisites. To tackle the problem, we proposed mixed-integer programming models, column generation approaches and parallel metaheuristic based algorithms.

In Chapter 3, we proposed five mixed-integer programming formulations CF1, CF2, EF1, $\mathrm{EF} 2$, and EF3. Formulations $\mathrm{CF} 1$ and $\mathrm{CF} 2$ can have their variables explicitly generated and solved in a black-box solver, while EF1, EF2, and EF3 cannot, because they have an exponential number of variables. Formulation $\mathrm{CF} 2$ has better linear relaxation than $\mathrm{CF} 1$, and we could also strengthen its root node with Fenchel cuts. The improved formulation CF2 outperformed the state-of-the-art formulation in the quality of lower bounds, but it is less effective in the quality of integer solutions. Formulations EF1, EF2, and EF3 are extended formulations solved by column generation, and their pricing subproblems are formulated with decompositions of formulation $\mathrm{CF} 2$. The best formulation EF2 provided strong lower bounds for the tested problems by expending cheap computational time. Also, it is competitive in the quality of lower bounds with extended formulations previously proposed in the literature. Although the formulations of Chapter 3 have worked quite well with Brazilian high school timetabling problems, they have a serious issue. As the number of binary variables is exponential on the number of periods per day, such formulations may be inefficient to deal with problems that require more than five periods per day.

In Chapter 4, we investigated several parallelization schemes to develop cooperative parallel metaheuristic based algorithms for high school timetabling problems. Such kind of algorithm has not been well studied in the context of educational timetabling. In our study, we analyzed several design aspects, such as homogeneity/heterogeneity of agents, the influence of intensification and diversification memories of solutions, and the frequency of information exchange among threads. The study showed that the exchange of information plays an important rule in the design of high-performance parallel metaheuristics. By running multiples copies of a 
cooperative metaheuristic in different processor threads of a multi-core machine, we can explore the total machine processing capacity and obtain consistent gains in performance when compared to the use of sequential metaheuristics. Our most efficient algorithm was obtained by including a diversification memory, a very elitist intensification memory and frequent exchange of solutions among threads. This algorithm was able to find near-optimal solutions for all tested problems and also outperformed state-of-the-art algorithms. This chapter resulted in the following research paper:

- Saviniec, L.; Santos, M. O.; Costa, A. M. Parallel local search algorithms for high school timetabling problems. European Journal of Operational Research, 265 (2018) $81-98$.

In Chapter 5, we combined the best column generation of Chapter 3 with the best strategies of Chapter 4 to develop a new parallel algorithm. This algorithm is able to compute lower bounds at the same time that it seeks for good quality solutions. The main feature of this algorithm is two embedded constructive heuristics, which exploit column generation information to construct feasible solutions that are further improved by a team of cooperative metaheuristics. Our experiments showed that these heuristics are quite effective in constructing feasible solutions, and by embedding them into our parallel framework, it resulted in an algorithm that is more effective than the best algorithm of Chapter 4 .

Chapters 3 and 5 resulted in a working paper. This thesis also generated two other conference papers that were published in the proceedings of the Brazilian Symposium on Operations Research, in which the paper "A Parallel Multi-start Iterated Local Search and a Proximity Relax-and-fix heuristic for High School Timetabling Problem" was select among the five best papers of the conference.

- Saviniec, L.; Santos, M. O.; Costa, A. M.; Constantino, A. A. Multithreading Iterated Local Search aplicado ao Problema de Horários Escolares. Brazilian Symposium on Operations Research, Sobrapo, Rio de Janeiro, Brazil (2015) 826 - 837. (In Portuguese)

- Saviniec, L.; Santos, M. O.; Costa, A. M. A Parallel Multi-start Iterated Local Search and a Proximity Relax-and-fix heuristic for High School Timetabling Problem. Brazilian Symposium on Operations Research, Sobrapo, Rio de Janeiro, Brazil (2017) 1 - 12.

\subsection{Directions for future research}

Below, we list some topics that can be further investigated in future studies, in order to extend this research.

In the line of exact methods for high school timetabling problems, a branch-and-price method can be devised, so that formulation EF2 of Chapter 3 is exploited to find the optimal 
solutions or improve the lower bounds for open instances. Moreover, as discussed before, formulation $\mathrm{CF} 2$ suffers a serious issue related to the exponential growth of binary variables, which depends on the number of periods per day. Therefore, the development of alternative formulations is also another possibility for future investigations. During this research, we have also investigated Benders decomposition for high school timetabling problems by using both compact formulations $\mathrm{CF} 1$ and $\mathrm{CF} 2$ of Chapter 3, but no exciting results were obtained. However, further investigations can be done if new formulations are developed.

Parallel metaheuristics is also a promising line of research in high school timetabling problems. In this thesis, we have developed parallel algorithms that exploit only trajectorybased metaheuristics as cooperative agents. Further studies to investigate cooperation among trajectory and population-based metaheuristics are also aimed. Another interesting research topic is the extension of the parallel algorithms proposed in Chapters 4 and 5 to run in a distributed environment. Adjust them to solve other timetabling problems, such as the university course and examination timetabling problems, is also another possible topic. 

ABDULLAH, S. Heuristic approaches for university timetabling problems. Phd Thesis (PhD Thesis) - The School of Computer Science and Information Technology - University of Nottingham, Nottingham, 2006. Citation on page 33.

ABRAMSON, D. Constructing school timetables using simulated annealing: sequential and parallel algorithms. Management science, INFORMS, v. 37, n. 1, p. 98-113, 1991. Citations on pages $27,40,87$ e 89 .

ABRAMSON, D.; ABELA, J. A parallel genetic algorithm for solving the school timetabling problem. Proceedings of the 15 Australian Computer Science Conference, Hobart, Australia, p. 1-11, 1992. Citations on pages 27, 87 e 89.

AHMED, L. N.; ÖZCAN, E.; KHEIRI, A. Solving high school timetabling problems worldwide using selection hyper-heuristics. Expert Systems with Applications, v. 42, n. 13, p. 5463-5471, 2015. ISSN 0957-4174. Citation on page 44.

AL-YAKOOB, S. M.; SHERALI, H. D. Mathematical models and algorithms for a high school timetabling problem. Computers \& Operations Research, v. 61, p. 56-68, 2015. ISSN 03050548. Citations on pages 42 e 44.

ALBA, E.; LUQUE, G.; NESMACHNOW, S. Parallel metaheuristics: recent advances and new trends. International Transactions in Operational Research, v. 20, n. 1, p. 1-48, 2013. ISSN 1475-3995. Citations on pages 88 e 89.

ALVAREZ-VALDÉS, R.; PARREÑO, F.; TAMARIT, J. M. A tabu search algorithm for assigning teachers to courses. Top, v. 10, n. 2, p. 239-259, 2002. ISSN 1863-8279. Citation on page 36.

AVELLA, P.; D’AURIA, B.; SALERNO, S.; VASIL'EV, I. A computational study of local search algorithms for italian high-school timetabling. Journal of Heuristics, v. 13, n. 6, p. 543-556, 2007. ISSN 1572-9397. Citations on pages 25, 36, 40, 41 e 43.

BELIGIANNIS, G. N.; MOSCHOPOULOS, C.; LIKOTHANASSIS, S. D. A genetic algorithm approach to school timetabling. Journal of the Operational Research Society, v. 60, n. 1, p. 23-42, 2009. ISSN 1476-9360. Citation on page 42.

BELIGIANNIS, G. N.; MOSCHOPOULOS, C. N.; KAPERONIS, G. P.; LIKOTHANASSIS, S. D. Applying evolutionary computation to the school timetabling problem: The greek case. Computers \& Operations Research, v. 35, n. 4, p. 1265-1280, 2008. ISSN 0305-0548. Citations on pages $36,41,42$ e 43 .

BELLO, G.; RANGEL, M.; BOERES, M. An approach for the class/teacher timetabling problem using graph coloring. In: The Practice and Theory of Automated Timetabling VII. [S.l.: s.n.], 2008. p. 1-6. Citation on page 43.

BIRBAS, T.; DASKALAKI, S.; HOUSOS, E. School timetabling for quality student and teacher schedules. Journal of scheduling, Springer, v. 12, n. 2, p. 177-197, 2009. Citations on pages 25,36 e 43. 
BONUTTI, A.; CESCO, F. D.; GASPERO, L. D.; SCHAERF, A. Benchmarking curriculumbased course timetabling: formulations, data formats, instances, validation, visualization, and results. Annals of Operations Research, Springer, v. 194, n. 1, p. 59-70, 2012. Citation on page 33 .

BOYD, E. A. Fenchel cutting planes for integer programs. Operations Research, v. 42, n. 1, p. 53-64, 1994. Citations on pages 27, 44 e 61.

BOYD, E. A. Solving 0/1 integer programs with enumeration cutting planes. Annals of Operations Research, v. 50, n. 1, p. 61-72, 1994. ISSN 1572-9338. Citations on pages 27, 44 e 61.

BOŻEJKO, W.; PEMPERA, J.; SMUTNICKI, C. Parallel tabu search algorithm for the hybrid flow shop problem. Computers \& Industrial Engineering, v. 65, n. 3, p. 466-474, 2013. ISSN 0360-8352. Citation on page 87.

BRITO, S. S.; FONSECA, G. H.; TOFFOLO, T. A.; SANTOS, H. G.; SOUZA, M. J. A sa-vns approach for the high school timetabling problem. Electronic Notes in Discrete Mathematics, v. 39, p. 169-176, 2012. ISSN 1571-0653. Citation on page 43.

BURKE, E.; ELLIMAN, D.; FORD, P.; WEARE, R. Examination timetabling in british universities: A survey. In: . Practice and Theory of Automated Timetabling: First International Conference Edinburgh, U.K., August 29-September 1, 1995 Selected Papers. Berlin, Heidelberg: Springer Berlin Heidelberg, 1996. p. 76-90. ISBN 978-3-540-70682-3. Citations on pages 34 e 35 .

BURKE, E. K.; BYKOV, Y. A late acceptance strategy in hill-climbing for exam timetabling problems. In: The 7th Conference on the Practice and Theory of Automated Timetabling, Montreal, Canada. [S.1.: s.n.], 2008. Citations on pages 89, 139 e 140.

CALDEIRA, J.; ROSA, A. School timetabling using genetic search. In: Practice and Theory of Automated Timetabling II. [S.1.]: Springer, 1997. p. 1-8. Citation on page 41.

CERDEIRA-PENA, A.; CARPENTE, L.; FARINA, A.; SECO, D. New approaches for the school timetabling problem. In: IEEE. Seventh Mexican International Conference on Artificial Intelligence (MICAI'08). [S.1.], 2008. p. 261-267. Citation on page 43.

CRAINIC, T. G.; GENDREAU, M.; HANSEN, P.; MLADENOVIĆ, N. Cooperative parallel variable neighborhood search for the p-median. Journal of Heuristics, v. 10, n. 3, p. 293-314, 2004. ISSN 1572-9397. Citation on page 90.

DEMIROVIć, E.; MUSLIU, N. Maxsat-based large neighborhood search for high school timetabling. Computers \& Operations Research, v. 78, p. 172-180, 2017. ISSN 0305-0548. Citation on page 44 .

DESAULNIERS, G.; DESROSIERS, J.; SOLOMON, M. M. Column generation. 1. ed. [S.1.]: Springer Science \& Business Media, 2005. Citations on pages 27 e 65.

DILLENBERGER, C.; ESCUDERO, L. F.; WOLLENSAK, A.; ZHANG, W. On practical resource allocation for production planning and scheduling with period overlapping setups. European Journal of Operational Research, v. 75, n. 2, p. 275-286, 1994. ISSN 0377-2217. Citation on page 28 . 
DORNELES, Á. P.; ARAÚJO, O. C. B. d.; BURIOL, L. S. The impact of compactness requirements on the resolution of high school timetabling problem. Proceedings of the Brazilian Symposium on Operations Research, Sobrapo, Rio de Janeiro, Brazil, p. 3336-3347, 2012. Citation on page 43.

DORNELES, Á. P.; ARAÚJO, O. C. de; BURIOL, L. S. A fix-and-optimize heuristic for the high school timetabling problem. Computers \& Operations Research, v. 52, Part A, p. 29-38, 2014. ISSN 0305-0548. Citations on pages 25, 26, 41, 42, 43, 45, 80, 87, 88 e 99.

A column generation approach to high school timetabling modeled as a multicommodity flow problem. European Journal of Operational Research, v. 256, n. 3, p. 685-695, 2017. ISSN 0377-2217. Citations on pages 26, 41, 42, 44, 45, 51, 80, 81, 82 e 85.

DRISCOLL, J. R.; SARNAK, N.; SLEATOR, D. D.; TARJAN, R. E. Making data structures persistent. Journal of Computer and System Sciences, v. 38, n. 1, p. 86-124, 1989. ISSN 0022-0000. Citation on page 134.

EVEN, S.; ITAI, A.; SHAMIR, A. On the complexity of timetable and multi-commodity flow problems. In: IEEE. 16th Annual Symposium on Foundations of Computer Science. [S.1.], 1975. p. 184-193. ISSN 0272-5428. Citations on pages 25 e 38.

FILHO, G. R.; LORENA, L. A. N. A constructive evolutionary approach to school timetabling. In: Applications of evolutionary computing. [S.1.]: Springer, 2001. p. 130-139. Citations on pages 40 e 43 .

FISCHETTI, M.; MONACI, M. Proximity search for 0-1 mixed-integer convex programming. Journal of Heuristics, v. 20, n. 6, p. 709-731, 2014. ISSN 1572-9397. Citations on pages 28, 107 e 110.

FONSECA, G. H.; SANTOS, H. G. Variable neighborhood search based algorithms for high school timetabling. Computers \& Operations Research, v. 52, Part B, p. 203-208, 2014. ISSN 0305-0548. Recent advances in Variable neighborhood search. Citations on pages 43 e 87.

FONSECA, G. H.; SANTOS, H. G.; CARRANO, E. G. Integrating matheuristics and metaheuristics for timetabling. Computers \& Operations Research, v. 74, p. 108-117, 2016. ISSN 0305-0548. Citations on pages 26, 42, 44, 87 e 100.

FONSECA, G. H.; SANTOS, H. G.; CARRANO, E. G.; STIDSEN, T. J. Integer programming techniques for educational timetabling. European Journal of Operational Research, v. 262, n. 1, p. 28-39, 2017. ISSN 0377-2217. Citations on pages 26, 42 e 44.

FONSECA, G. H. G.; SANTOS, H. G.; CARRANO, E. G. Late acceptance hill-climbing for high school timetabling. Journal of Scheduling, v. 19, n. 4, p. 453-465, 2016. ISSN 1099-1425. Citation on page 44 .

FONSECA, G. H. G. da; SANTOS, H. G.; TOFFOLO, T. Â. M.; BRITO, S. S.; SOUZA, M. J. F. Goal solver: a hybrid local search based solver for high school timetabling. Annals of Operations Research, v. 239, n. 1, p. 77-97, 2016. ISSN 1572-9338. Citations on pages 17, $26,42,44,87,88$ e 100.

GASPERO, L. D.; MCCOLLUM, B.; SCHAERF, A. The second international timetabling competition (ITC-2007): Curriculum-based course timetabling (track 3). [S.1.], 2007. Citations on pages 30,32 e 33 . 
GASPERO, L. D.; SCHAERF, A. Tabu search techniques for examination timetabling. In: Practice and Theory of Automated Timetabling III: Third International Conference, PATAT 2000 Konstanz, Germany, August 16-18, 2000 Selected Papers. Berlin, Heidelberg: Springer Berlin Heidelberg, 2001. p. 104-117. ISBN 978-3-540-44629-3. Citations on pages 34 e 35 .

GLOVER, F. Tabu search: A tutorial. Interfaces, v. 20, n. 4, p. 74-94, 1990. Citation on page 89.

GOTLIEB, C. C. The construction of class-teacher timetables. In: POPPLEWELL, C. M. (Ed.). Proceedings of the IFIP Congress 62 in Information Processing 1963. [S.l.: s.n.], 1963. p. 73-77. Citation on page 29.

HAAN, P. D.; LANDMAN, R.; POST, G.; RUIZENAAR, H. A case study for timetabling in a dutch secondary school. In: Practice and Theory of Automated Timetabling VI. [S.1.]: Springer, 2007. p. 267-279. Citation on page 41.

JACOBSEN, F.; BORTFELDT, A.; GEHRING, H. Timetabling at german secondary schools: tabu search versus constraint programming. In: CITESEER. Proceedings of the 6th International Conference on the Practice and Theory of Automated Timetabling. [S.1.], 2006. p. 439-442. Citations on pages 36, 40 e 43.

JIN, J.; CRAINIC, T. G.; LØKKETANGEN, A. A cooperative parallel metaheuristic for the capacitated vehicle routing problem. Computers \& Operations Research, v. 44, p. 33-41, 2014. ISSN 0305-0548. Citation on page 90.

JUNGINGER, W. Timetabling in germany - a survey. Interfaces, v. 16, n. 4, p. 66-74, 1986. Citations on pages 29, 39, 40 e 41 .

KAPLAN, H. Persistent data structures. In: MEHTA, D. P.; SAHNI, S. (Ed.). Handbook of data structures and applications. [S.1.]: Chapman \& Hall/CRC, 2005, (Chapman \& Hall/CRC computer and information science series). ISBN 978-1-58488-435-4. Citation on page 133.

KINGSTON, J. H. A software library for high school timetabling. 2015. Available: <http: //sydney.edu.au/engineering/it/ jeff/khe/>. Citations on pages 18 e 100.

KIRKPATRICK, S.; GELATT, C. D.; VECCHI, M. P. Optimization by simmulated annealing. Science, v. 220, n. 4598, p. 671-680, 1983. Citation on page 89.

KOCHETOV, Y.; KONONOVA, P.; PASCHENKO, M. Formulation space search approach for the teacher/class timetabling problem. Yugoslav Journal of Operations Research, v. 18, n. 1, p. 1-11, 2008. ISSN 0354-0243. Citations on pages 41 e 43.

KRISTIANSEN, S.; SØRENSEN, M.; STIDSEN, T. R. Integer programming for the generalized high school timetabling problem. Journal of Scheduling, v. 18, n. 4, p. 377-392, 2015. ISSN 1099-1425. Citations on pages 25, 42 e 44.

KRISTIANSEN, S.; STIDSEN, T. R. A Comprehensive Study of Educational Timetabling-a Survey. [S.1.], 2013. Citation on page 30.

LARA, C.; FLORES, J. J.; CALDERÓN, F. Solving a school timetabling problem using a bee algorithm. In: MICAI 2008: Advances in Artificial Intelligence: 7th Mexican International Conference on Artificial Intelligence. [S.1.]: Springer Berlin Heidelberg, 2008. p. 664-674. ISBN 978-3-540-88636-5. Citation on page 43. 
LEWIS, R. A survey of metaheuristic-based techniques for university timetabling problems. OR Spectrum, Springer, v. 30, n. 1, p. 167-190, 2008. Citations on pages 25 e 30.

LEWIS, R.; PAECHTER, B.; MCCOLLUM, B. et al. Post enrolment based course timetabling: A description of the problem model used for track two of the second international timetabling competition. Cardiff Business School, 2007. Citations on pages 30 e 32.

LOURENÇO, H. R.; MARTIN, O. C.; STÜTZLE, T. Iterated local search. In: Handbook of Metaheuristics. Boston, MA: Springer US, 2003. p. 320-353. ISBN 978-0-306-48056-0. Citation on page 89.

LÜ, Z.; HAO, J.; GLOVER, F. Neighborhood analysis: a case study on curriculum-based course timetabling. Journal of Heuristics, v. 17, n. 2, p. 97-118, 2011. ISSN 1572-9397. Citation on page 134 .

LUQUE, G.; ALBA, E. Parallel hybrid trajectory based metaheuristics for real-world problems. In: IEEE. International Conference on Intelligent Networking and Collaborative Systems (INCOS). [S.1.], 2015. p. 184-191. Citations on pages 87 e 89.

MARTE, M. Towards constraint-based school timetabling. Annals of Operations Research, v. 155, n. 1, p. 207-225, 2007. ISSN 1572-9338. Citations on pages 40 e 43.

MCCOLLUM, B.; MCMULLAN, P.; BURKE, E. K.; PARKES, A. J.; QU, R. The second international timetabling competition: Examination timetabling track. [S.1.], 2007. Citation on page 34 .

MELICIO, F.; CALDEIRA, J.; ROSA, A. Two neighbourhood approaches to the timetabling problem. In: BURKE, E.; TRICK, M. (Ed.). Practice and theory of automated timetabling V. [S.1.]: Springer, 2004. v. 3616, p. 267-282. Citation on page 40.

NURMI, K.; KYNGÄS, J. A framework for school timetabling problem. In: Proceedings of the 3rd multidisciplinary international scheduling conference: theory and applications. [S.1.: s.n.], 2007. p. 386-393. Citation on page 40.

PAPOUTSIS, K.; VALOUXIS, C.; HOUSOS, E. A column generation approach for the timetabling problem of greek high schools. Journal of the Operational Research Society, v. 54, n. 3, p. 230-238, 2003. ISSN 1476-9360. Citation on page 26.

PETROVIC, S.; BURKE, E. University timetabling. In: CITESEER. Handbook of Scheduling: Algorithms, Models, and Performance Analysis, chapter 45. [S.1.], 2004. Citation on page 32.

PILLAY, N. A study into the use of hyper-heuristics to solve the school timetabling problem. In: Proceedings of the 2010 Annual Research Conference of the South African Institute of Computer Scientists and Information Technologists. [S.1.: s.n.], 2010. p. 258-264. Citation on page 43.

A survey of school timetabling research. Annals of Operations Research, Springer, v. 218 , n. 1, p. 261-293, 2014. Citations on pages 25, 40, 43 e 87.

POST, G.; AHMADI, S.; DASKALAKI, S.; KINGSTON, J.; KYNGAS, J.; NURMI, C.; RANSON, D. An xml format for benchmarks in high school timetabling. Annals of Operations Research, Springer, v. 194, n. 1, p. 385-397, 2012. Citations on pages 25 e 42. 
POST, G.; AHMADI, S.; GEERTSEMA, F. Cyclic transfers in school timetabling. OR Spectrum, v. 34, n. 1, p. 133-154, 2012. ISSN 1436-6304. Citations on pages 36 e 40.

POST, G.; GASPERO, L. D.; KINGSTON, J. H.; MCCOLLUM, B.; SCHAERF, A. The third international timetabling competition. Annals of Operations Research, v. 239, n. 1, p. 69-75, 2016. Citation on page 30 .

POST, G.; KINGSTON, J. H.; AHMADI, S.; DASKALAKI, S.; GOGOS, C.; KYNGAS, J.; NURMI, C.; MUSLIU, N.; PILLAY, N.; SANTOS, H.; SCHAERF, A. Xhstt: an xml archive for high school timetabling problems in different countries. Annals of Operations Research, v. 218, n. 1, p. 295-301, 2014. ISSN 1572-9338. Citation on page 42.

QU, R.; BURKE, E. K.; MCCOLLUM, B.; MERLOT, L. T.; LEE, S. Y. A survey of search methodologies and automated system development for examination timetabling. Journal of scheduling, Springer, v. 12, n. 1, p. 55-89, 2009. Citation on page 25.

RAGHAVJEE, R.; PILLAY, N. An application of genetic algorithms to the school timetabling problem. In: Proceedings of the 2008 annual research conference of the South African Institute of Computer Scientists and Information Technologists on IT research in developing countries: riding the wave of technology. [S.1.: s.n.], 2008. p. 193-199. Citation on page 43.

Evolving solutions to the school timetabling problem. In: IEEE. 2009 World Congress on Nature \& Biologically Inspired Computing (NaBIC). [S.1.], 2009. p. 1524-1527. Citation on page 43 .

An informed genetic algorithm for the high school timetabling problem. In: Proceedings of the 2010 Annual Research Conference of the South African Institute of Computer Scientists and Information Technologists. [S.1.: s.n.], 2010. p. 408-412. Citation on page 43.

Using genetic algorithms to solve the south african school timetabling problem. In: IEEE. 2010 Second World Congress on Nature and Biologically Inspired Computing (NaBIC). [S.1.], 2010. p. 286-292. Citation on page 43.

SÁNCHEZ-ORO, J.; SEVAUX, M.; ROSSI, A.; MARTÍ, R.; DUARTE, A. Solving dynamic memory allocation problems in embedded systems with parallel variable neighborhood search strategies. Electronic Notes in Discrete Mathematics, v. 47, p. 85-92, 2015. ISSN 1571-0653. Citation on page 87.

SANTOS, H.; OCHI, L.; SOUZA, M. A tabu search heuristic with efficient diversification strategies for the class/teacher timetabling problem. Journal of Experimental Algorithmics, ACM, v. 10, p. 2-9, 2005. ISSN 1084-6654. Citations on pages 41, 43, 44, 80 e 81.

SANTOS, H.; UCHOA, E.; OCHI, L.; MACULAN, N. Strong bounds with cut and column generation for class-teacher timetabling. Annals of Operations Research, Springer, v. 194, n. 1, p. 399-412, 2012. Citations on pages 25, 26, 41, 42, 43, 44, 45, 61, 80, 81 e 85.

SANTOS, L. M. R. dos; MICHELON, P.; ARENALES, M. N.; SANTOS, R. H. S. Crop rotation scheduling with adjacency constraints. Annals of Operations Research, v. 190, n. 1, p. 165-180, 2011. ISSN 1572-9338. Citation on page 107.

SAVINIEC, L. Operadores de vizinhança eficientes para algoritmos de busca local aplicados ao problema de horários em escolas. Master's Thesis (Master's Thesis) — Programa de Pós-Graduação em Ciência da Computação - Universidade Estadual de Maringá, Maringá, 2013. Citations on pages $18,41,45,51,52$ e 53 . 
SAVINIEC, L.; CONSTANTINO, A. A. Effective local search algorithms for high school timetabling problems. Applied Soft Computing, v. 60, p. 363-373, 2017. ISSN 1568-4946. Citations on pages 26, 41, 42, 44, 45, 73, 87, 105 e 134.

SAVINIEC, L.; CONSTANTINO, A. A.; ROMÃO, W.; SANTOS, H. G. Solving the high school timetabling problem to optimality by using ils algorithms. Proceedings of the Brazilian Symposium on Operations Research, Sobrapo, Rio de Janeiro, Brazil, p. 3330-3341, 2013. Citations on pages 41, 42, 43, 45, 80, 88 e 99.

SAVINIEC, L.; SANTOS, M. O.; COSTA, A. M. Parallel local search algorithms for high school timetabling problems. European Journal of Operational Research, v. 265, n. 1, p. 81-98, 2018. ISSN 0377-2217. Citation on page 87.

SCHAERF, A. Local search techniques for large high school timetabling problems. IEEE Transactions on Systems, Man and Cybernetics, Part A: Systems and Humans, IEEE, v. 29, n. 4, p. 368-377, 1999. ISSN 1083-4427. Citations on pages 40 e 41.

A survey of automated timetabling. Artificial Intelligence Review, v. 13, n. 2, p. 87-127, 1999. ISSN 1573-7462. Citations on pages 29, 30, 31, 33, 34, 38 e 40.

SKOULLIS, V. I.; TASSOPOULOS, I. X.; BELIGIANNIS, G. N. Solving the high school timetabling problem using a hybrid cat swarm optimization based algorithm. Applied Soft Computing, v. 52, p. 277-289, 2017. ISSN 1568-4946. Citations on pages 26, 42, 44 e 87.

SØRENSEN, M.; STIDSEN, T. R. Hybridizing integer programming and metaheuristics for solving high school timetabling. In: 10th international conference on the practice and theory of automated timetabling. [S.1.: s.n.], 2014. p. 557-560. Citation on page 43.

SOUSA, V.; MORETTI, A.; PODESTÁ, V. Programação da grade de horário em escolas de ensino fundamental e médio. Pesquisa Operacional, Scielo, v. 28, n. 3, p. 399-421, 2008. ISSN 0101-7438. Citations on pages 35, 36, 40, 43 e 51.

SOUZA, M. J. F. Programação de Horários em Escolas: Uma Aproximação por Metaheurísticas. Phd Thesis (PhD Thesis) — Programa de Pós-Graduação em Engenharia de Sistemas e Computação - Universidade Federal do Rio de Janeiro, Rio de Janeiro, 2000. Citations on pages $15,18,25,41,42,43,44,45,51,52,54,55,67,70,72,80$ e 81 .

SOUZA, M. J. F.; MACULAN, N.; OCHI, L. S. A grasp-tabu search algorithm for solving school timetabling problems. In:__. Metaheuristics: Computer Decision-Making. [S.1.]: Springer US, 2004. p. 659-672. ISBN 978-1-4757-4137-7. Citations on pages 25, 35, 36, 40, 41, 43, 44 e 80 .

SRNDIC, N.; PANDZO, E.; DERVISEVIC, M.; KONJICIJA, S. The application of a parallel genetic algorithm to timetabling of elementary school classes: A coarse grained approach. In: IEEE. Information, Communication and Automation Technologies, 2009. ICAT 2009. XXII International Symposium on. [S.1.], 2009. p. 1-5. Citations on pages 27, 87 e 89.

STEFANO, C. D.; TETTAMANZI, A. An evolutionary algorithm for solving the school timetabling problem. In: Proceedings of the EvoWorkshops on Applications of Evolutionary Computing. [S.1.]: Springer-Verlag, 2001. p. 452-462. ISBN 3-540-41920-9. Citations on pages 40 e 43 . 
SUBRAMANIAN, A.; DRUMMOND, L.; BENTES, C.; OCHI, L.; FARIAS, R. A parallel heuristic for the vehicle routing problem with simultaneous pickup and delivery. Computers $\&$ Operations Research, v. 37, n. 11, p. 1899-1911, 2010. ISSN 0305-0548. Citation on page 87.

SøRENSEN, M.; DAHMS, F. H. A two-stage decomposition of high school timetabling applied to cases in denmark. Computers \& Operations Research, v. 43, p. 36-49, 2014. ISSN 03050548. Citations on pages 25,42 e 43 .

TASSOPOULOS, I. X.; BELIGIANNIS, G. N. A hybrid particle swarm optimization based algorithm for high school timetabling problems. Applied Soft Computing, v. 12, n. 11, p. 3472-3489, 2012. ISSN 1568-4946. Citations on pages 26, 36, 41 e 42.

Solving effectively the school timetabling problem using particle swarm optimization. Expert Systems with Applications, v. 39, n. 5, p. 6029-6040, 2012. ISSN 0957-4174. Citation on page 41 .

VALOUXIS, C.; HOUSOS, E. Constraint programming approach for school timetabling. Computers \& Operations Research, v. 30, n. 10, p. 1555-1572, 2003. ISSN 0305-0548. Part Special Issue: Analytic Hierarchy Process. Citations on pages 35 e 43.

WERRA, D. D. Construction of school timetables by flow methods. INFOR: Information Systems and Operational Research, v. 9, n. 1, p. 12-22, 1971. Citation on page 38.

An introduction to timetabling. European Journal of Operational Research, Elsevier, v. 19, n. 2, p. 151-162, 1985. Citations on pages 25, 31, 38 e 40.

WILKE, P.; OSTLER, J. Solving the school time tabling problem using tabu search, simulated annealing, genetic and branch \& bound algorithms. In: Proceedings of the 7th International Conference on the Practice and Theory of Automated Timetabling. [S.1.: s.n.], 2008. p. 1-4. Citations on pages 36, 40 e 43 .

ZHANG, D.; LIU, Y.; M'HALLAH, R.; LEUNG, S. C. A simulated annealing with a new neighborhood structure based algorithm for high school timetabling problems. European Journal of Operational Research, v. 203, n. 3, p. 550-558, 2010. ISSN 0377-2217. Citations on pages 40, 41, 42 e 43. 
APPENDIX

\section{A}

\section{DESCRIPTION AND TUNING OF THE STAND-ALONE METAHEURISTICS}

In this appendix, we describe the sequential metaheuristics used in Chapter 4. We describe the data structure proposed to encode timetables, the neighborhood structure, the heuristic to construct initial solutions and the objective function evaluation. The pseudo-code for each metaheuristic and tuning procedures are also discussed.

\section{A.1 Timetable encoding}

Timeslots array $S$

\begin{tabular}{|c|c|c|c|c|c|}
\hline$s_{0}$ & $s_{1}$ & $\cdots$ & $S_{|D \times H|-2}$ & \multicolumn{2}{|c|}{$S_{|D \times H|-1}$} \\
\hline$c_{0}$ & $c_{0}$ & & & & $c_{0}$ \\
\hline$c_{1}$ & $c_{1}$ & & & & $c_{1}$ \\
\hline : & : & & & & : \\
\hline$c_{|C|-2}$ & $c_{|C|-2}$ & & & & $c_{|C|-2}$ \\
\hline$c_{|C|-1}$ & $c_{|C|-1}$ & & & & $c_{|C|-1}$ \\
\hline
\end{tabular}

Figure 18 - Proposed timetable encoding for the HSTP.

Trajectory-based metaheuristics frequently modify copies of the current or global best solution. However, some algorithms do not modify the whole copy, keeping parts that are identical to that of the previous solution. To avoid the duplication of unnecessary data (parts of the data structure that are copied but not modified), we designed a new timetable encoding to HSTP based on persistent data structures (KAPLAN, 2005). In a persistent data structure, each modification generates a new version of the structure, keeping the previous version accessible. There are several consolidated methods to make a data structure persistent (DRISCOLL et al., 
1989). One of these methods is based on structural sharing. The idea is to share with the new copy, parts of the original structure that remain unchanged.

To apply this approach, we designed the encoding shown in Figure 18, in which $S$ represents an array of all timeslots $(d, h) \in D \times H$. Each position of $S$ points to an array of integer values. In each of these arrays, the first position stores the ID of the teacher assigned to class 0 , the second position stores the teacher assigned to class 1 , and so on. The next section explains how this timetable encoding works with neighborhoods.

\section{A.2 Neighborhood structure}

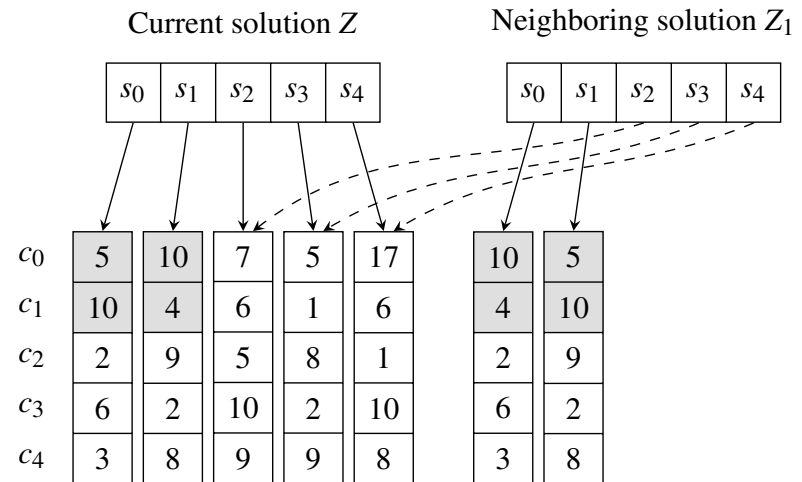

(a) Example of a TQ move applied to a partial solution $Z$ showing the generated neighboring solution $Z_{1}$.

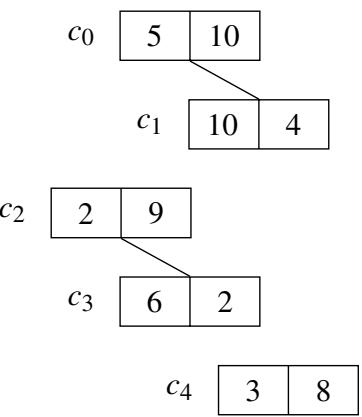

(b) Conflict graph for lessons assigned to timeslots $s_{0}$ and $s_{1}$ of solution $Z$, in Figure 19a, showing three possible moves.

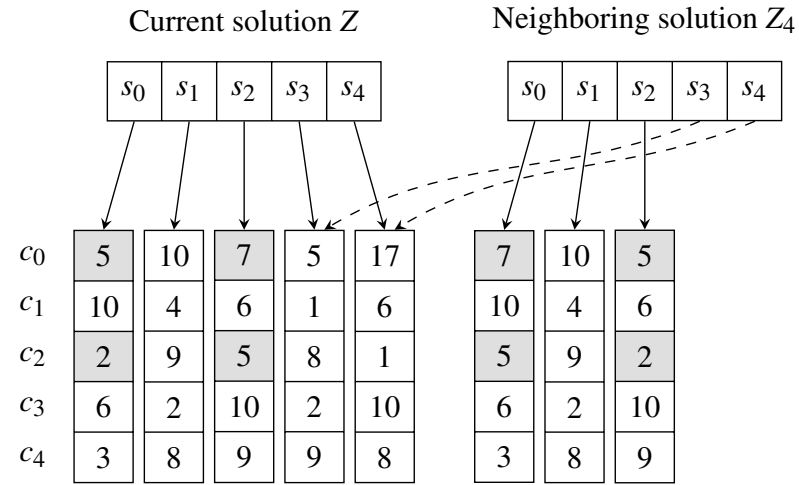

(c) Example of a fourth move, supposing that the three previous moves did not improve the current solution $Z$.

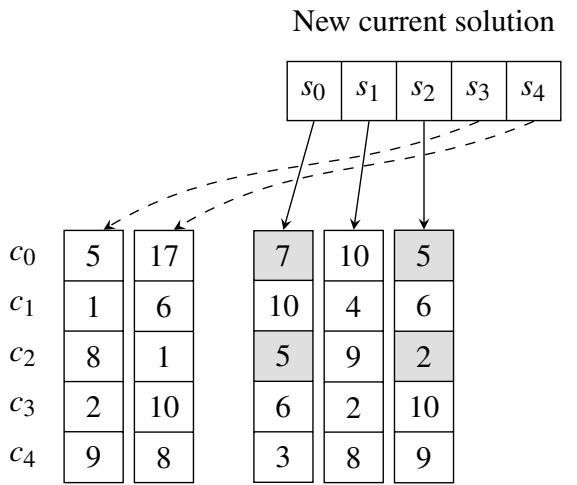

(d) The new current solution, supposing that the fourth move improved $Z$.

Figure 19 - The TQ neighborhood generation with the proposed timetable encoding.

We use a two-swap operator which, for a given class $c \in C$, exchanges the teachers assigned to two different timeslots. Feasibility in hard requisites 1 (meeting of weekly required lessons), 2 (no clashes in classes' schedules) and 3 (no clashes in teachers' schedules) is maintained with the use of the Torque Neighborhood Operator (TQ) proposed in Saviniec and Constantino (2017). The TQ acts as a Kempe Chain Interchange (LÜ; HAO; GLOVER, 2011) 
that uses the idea of conflict graph and effects a series of consecutive swaps until there are no clashes in teachers nor classes' schedules.

Figure 19 shows examples of TQ moves with the encoding scheme proposed in Section A.1. The TQ operator is applied to a partial solution $Z$ shown in Figure 19a. The conflict graph in Figure 19b identifies valid moves (connected components) in order to avoid clashes. The teachers assigned to classes $c_{0}$ and $c_{1}$ are swapped between timeslots $s_{0}$ and $s_{1}$. In the generated neighboring solution $Z_{1}$, timeslots $s_{0}$ and $s_{1}$ point to new arrays while remaining timeslots share the current arrays. Note that the arrays not changed in the move are shared between both solutions. Figures $19 \mathrm{c}$ and $19 \mathrm{~d}$ show that to operate or modify only timeslots $s_{0}, s_{1}$ and $s_{2}$, we do not need to copy the whole solution $Z$.

We employ this neighborhood generation scheme in all sequential and parallel metaheuristics proposed in this Thesis.

\section{A.3 Initial solutions}

Our initial solutions are constructed by the randomized heuristic described in Algorithm 7. This heuristic receives an input list $L$ of pairs $(c \in C, t \in T)$, in which each entry represents one lesson to be scheduled. At each iteration, the heuristic selects a lesson from the list and assigns it to a timeslot that is randomly selected among the free timeslots. This heuristic always generates solutions that are feasible for hard requisites 1 and 2.

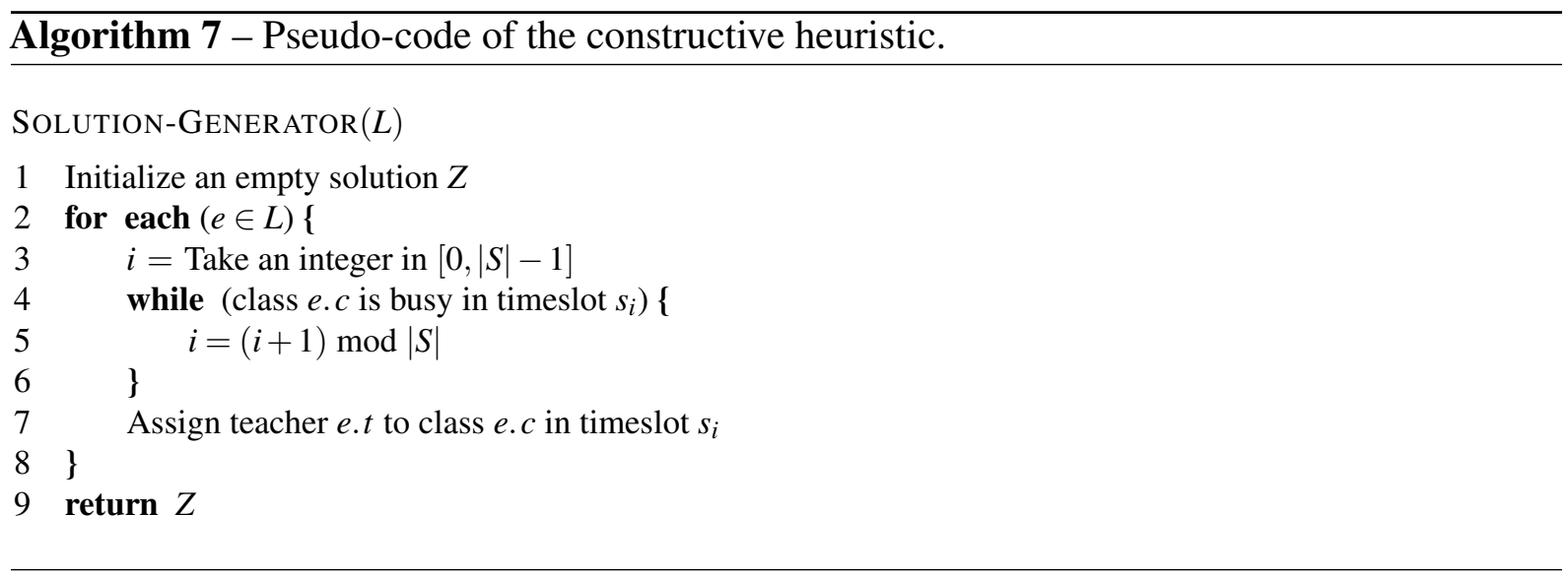

\section{A.4 Objective function evaluation}

Our metaheuristics are allowed to work in an infeasible space by relaxing the hard requisites, except 1 and 2, and penalizing them in the objective function with high penalties to enforce feasibility. Let $R_{\#}$ bet the set of requisites (not including 1 and 2) of problem \#, for \# 
representing HSTP-A, HSTP-B or HSTP-C. The objective function of problem \# is given by:

$$
\text { Minimize } \quad f(Z)=\sum_{i \in R_{\#}} \alpha_{i} V_{i}
$$

Where $\alpha_{i}$ and $V_{i}$ are the penalty and the number of violations made on requisite $i \in R_{\#}$, respectively.

As the constructive heuristic (Algorithm 7) always generates feasible solutions to hard requisites 1 and 2, and this feasibility is maintained by the neighborhood operator TQ, then only the remaining requisites are explicitly considered in the objective function. We used the following penalty values for violations of the hard requisites. Problem HSTP-A ( $\left.\alpha_{3}=\alpha_{4}=100000\right)$, problem HSTP-B $\left(\alpha_{3}=\alpha_{4}=100000, \alpha_{5}=10000\right)$, and problem HSTP-C ( $\alpha_{3}=\alpha_{4}=100000$, $\left.\alpha_{5}=10000, \alpha_{6}=5000\right)$.

In our implementation, we use incremental evaluation of solutions. The algorithms are designed to evaluate only the changed parts whenever a new move is performed.

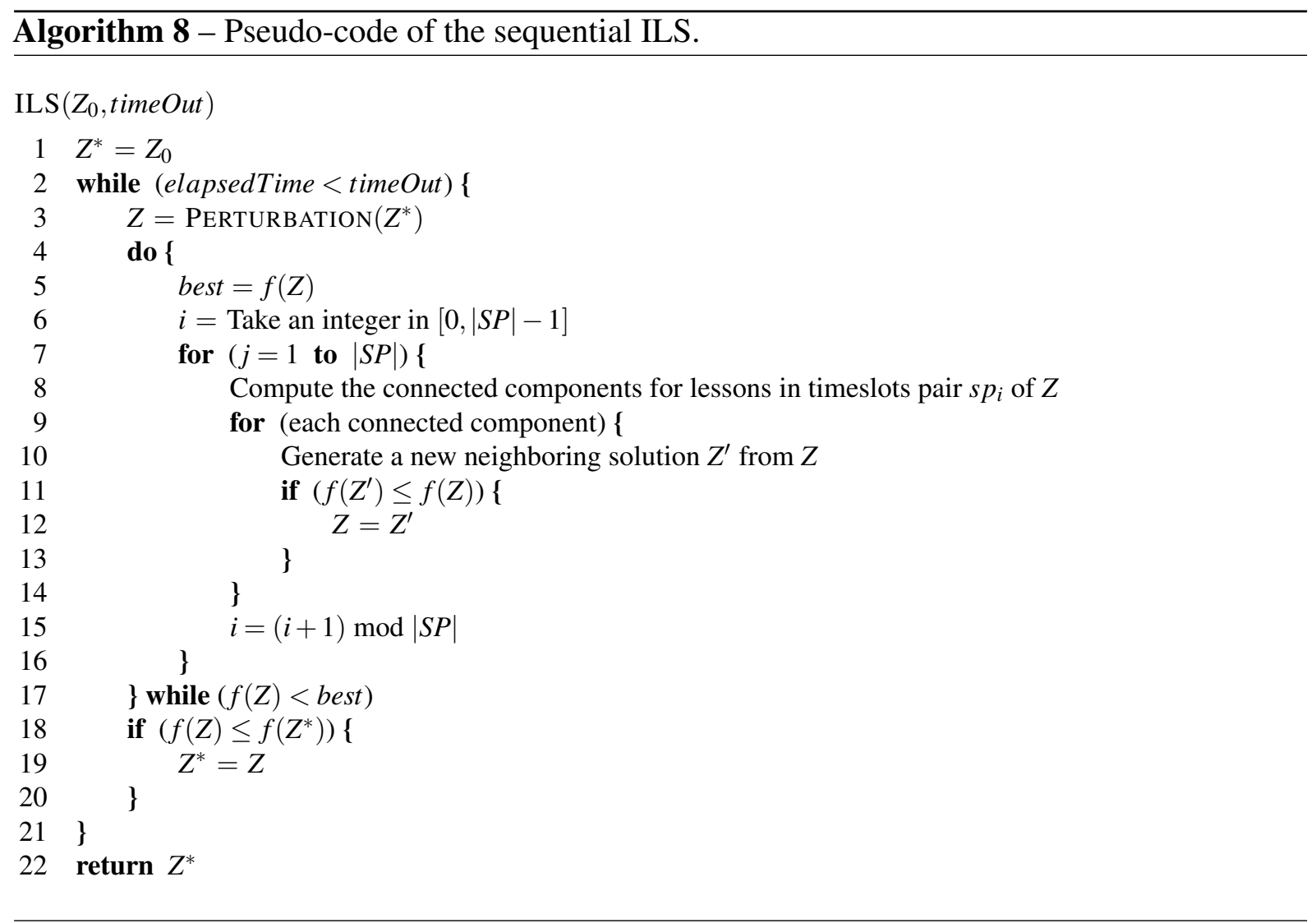

\section{A.5 Sequential metaheuristics for high school timetabling}

Our sequential metaheuristics to the HSTP include versions of iterated local search (ILS), tabu search (TS), simulated annealing (SA) and late acceptance strategy (LAS). 
We define $S P$ as the array of all timeslot pairs $\left(s_{i}, s_{j}\right)$, for $i, j=0, \ldots,|S|-1$ and $i \neq j$. Therefore, neighborhoods are defined by the way in which the timeslot pairs are explored in $S P$. Each of our algorithms may search this array using a different strategy.

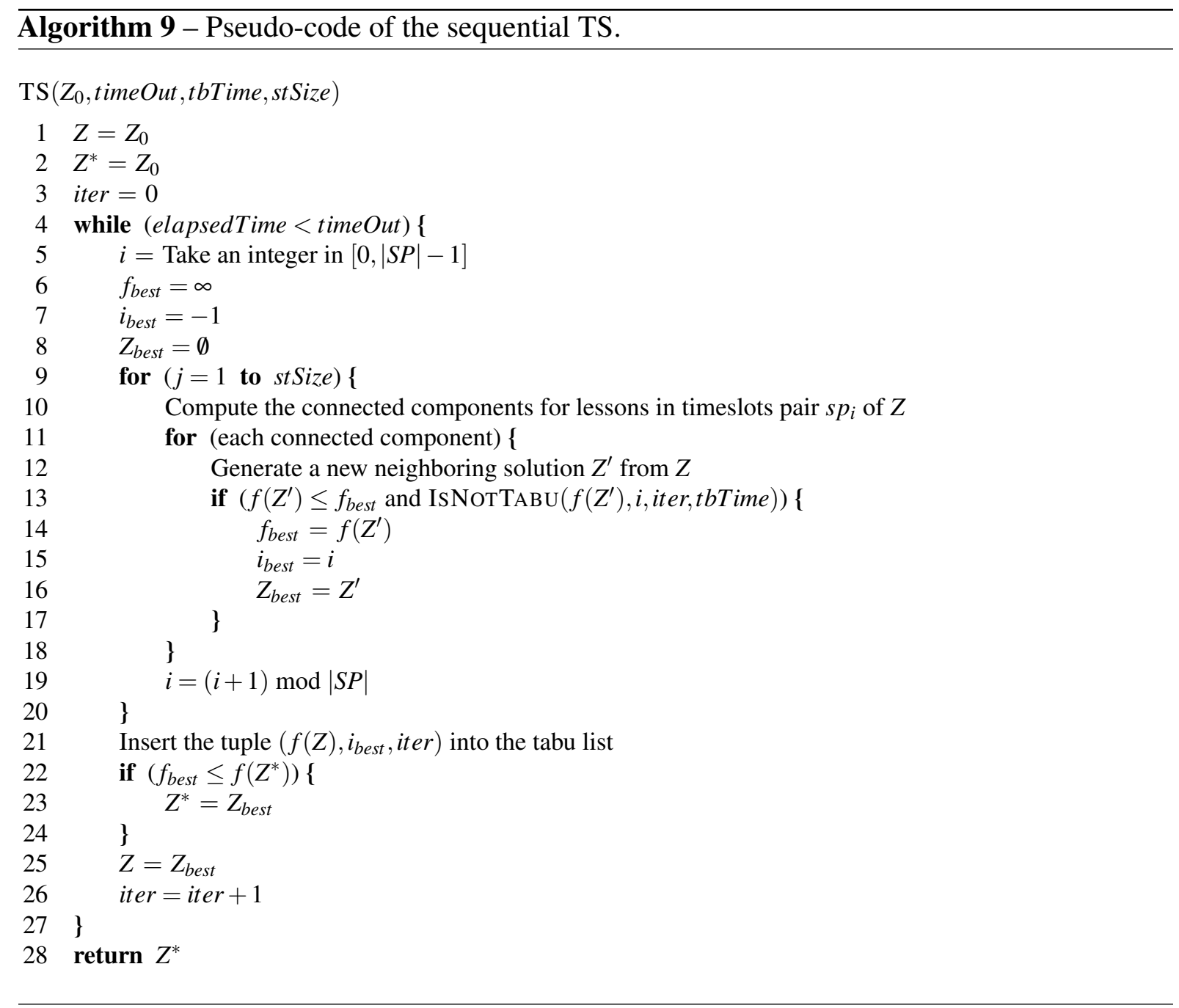

\section{A.5.1 Iterated local search}

The pseudo-code of the implemented sequential iterated local search is shown in Algorithm 8. At each iteration of the outer loop (from line 2 to line 21), it makes a random perturbation (line 3 ) in the current solution, followed by a local search (loop from line 4 to line 17). The local search starts in a random index $i$ of $S P$ and evaluates the next $|S P|$ consecutive timeslot pairs. For each index, it generates the connected components according to what was discussed in Section A.2. If it finds a connected component that generates an improved solution, then the new solution is accepted. These steps are repeated while an improved solution is found after exploring $|S P|$ consecutive indexes ${ }^{1}$. The PERTURBATION procedure, in line 3 , returns a random neighboring solution of the global best solution $Z^{*}$. This procedure selects a random index $k$ of

1 The algorithm iterates through the indexes of $S P$ in a circular fashion as in a circular linked list. 
$S P$, constructs the conflict graph for lessons assigned in timeslots $s p_{k}$ of the solution $Z^{*}$, and makes a random TQ move with the associated connected components.

\section{A.5.2 Tabu search}

The pseudo-code of the implemented sequential tabu search is shown in Algorithm 9. At each iteration of the outer loop (from line 4 to 27), the algorithm searches the best neighbor of the current solution $Z$. The best neighbor is accepted if it is better or equal to the global best solution $Z^{*}$. The search starts in a random index $i$ of $S P$, as in the ILS, and evaluates a strip of $S P$ defined by the next stSize consecutive timeslot pairs. Only moves that are not tabu are accepted. A tabu move is a tuple ( $f$, index, iter), where $f$ is the objective function value before the move, index is the index of SP in which the move was done, and iter is the iteration in which the move was performed. The parameter tbTime is the number of iterations in which a move remains tabu (the aspiration criterion). The procedure ISNOTTABU (line 13) checks if a move is tabu and also, removes those moves in which the tabu time has expired.

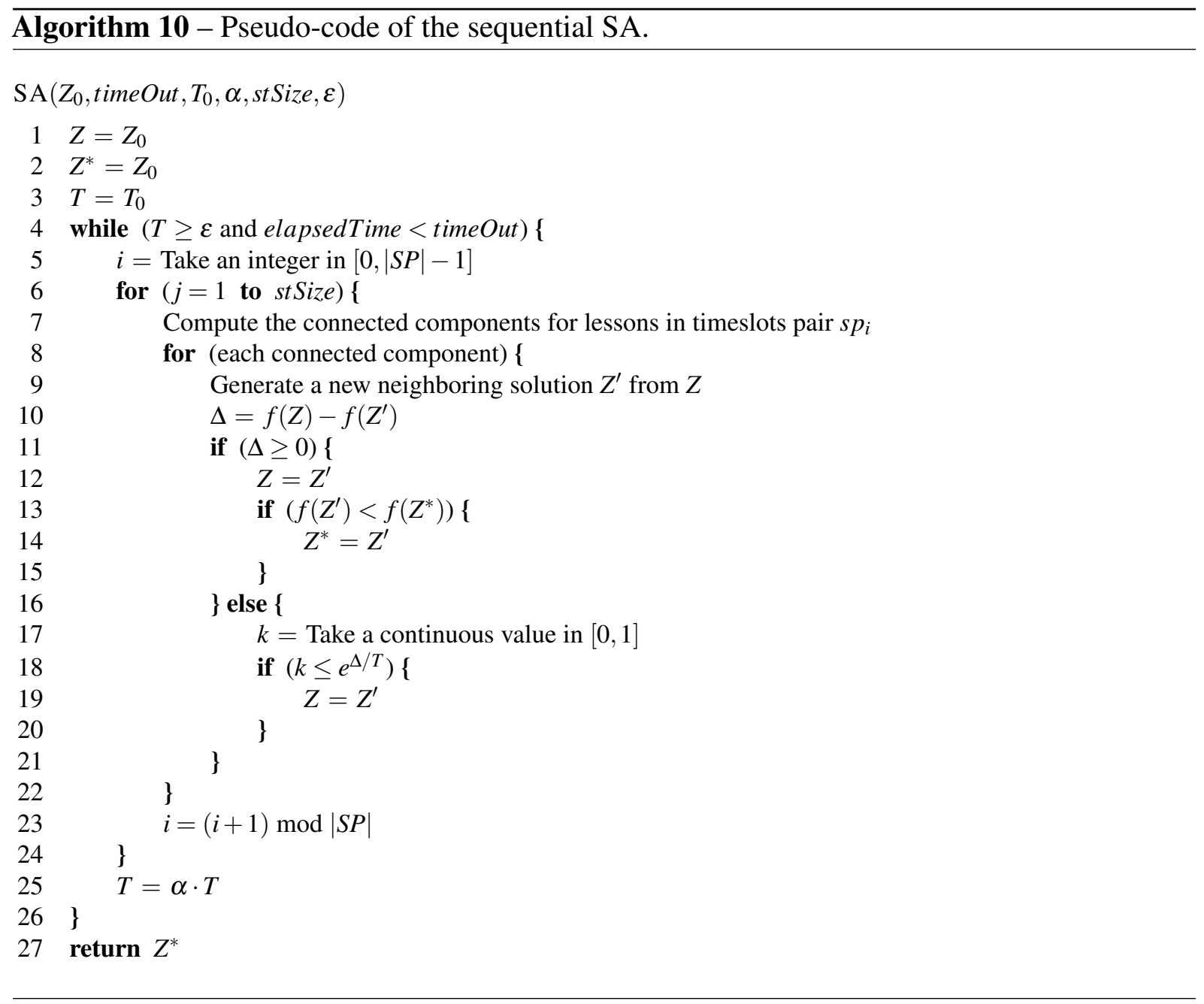




\section{A.5.3 Simulated annealing}

The pseudo-code of the implemented sequential simulated annealing is shown in Algorithm 10. The code follows a standard SA framework. However, instead of selecting each new neighboring solution randomly, as in standard SA, the algorithm chooses a random index of $S P$, as a starting point, and searches the next st Size consecutive timeslot pairs. The outer loop (from line 4 to 26) controls the temperature level and stops after a time-out is reached or the temperature $T$ reaches a small value given by parameter $\varepsilon$. The remaining steps are those from a standard SA framework. Parameters $T_{0}$ and $\alpha$ are the initial temperature and the cooling rate, respectively.

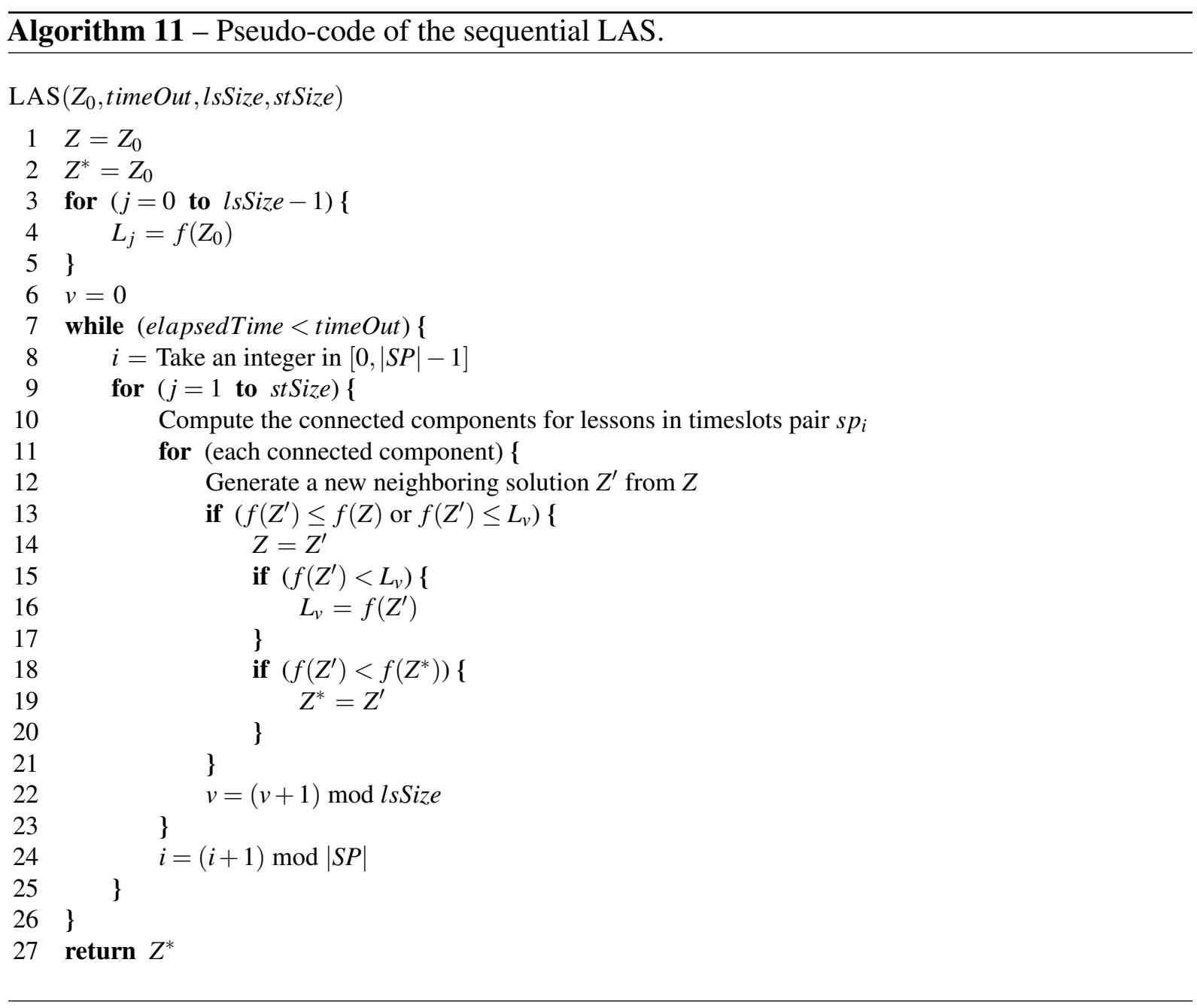

\section{A.5.4 Late acceptance strategy}

The last sequential metaheuristic proposed is a late acceptance strategy, for which a pseudo-code is presented in Algorithm 11. The LAS is a recent metaheuristic approach (BURKE; BYKOV, 2008) that maintains a list $L$ of size $l s$ Size which records the objective values of the last $l$ sSize accepted solutions. The main idea of the LAS is to compare the current solution with 
a previous current solution recorded in L (BURKE; BYKOV, 2008). In our version of the LAS, the neighborhood is searched by strips, as in our versions of TS and SA described above.

\section{A.6 Parameter tuning}

In this section, we report the computational experiments carried out to calibrate the sequential metaheuristics described in Section A.5.

To these experiments, we selected the problem HSTP-A and a subset of the 34 instances from Table 15 to analyze and tune the sequential metaheuristics. The reduced set of instances comprises two small (10 and 13), two medium (6 and 26) and two large (7 and 24) instances.

Figures 20-23 present charts of normalized objective values over time for different algorithm configurations. The $x$-axis (time in seconds) is in quadratic scale. Objective values were collected for each time $x=i^{2}$ seconds, for $i=1, \ldots, 25$. The $y$-axis are normalized objective values obtained as follows. Let $Z_{j}$ be a timetable solution for the $j^{\text {th }}$ instance, its normalized objective value is given by $\operatorname{NOV}\left(Z_{j}\right)=f\left(Z_{j}\right) / f\left(Z_{j}^{*}\right)$. Where $f\left(Z_{j}^{*}\right)$ is a benchmark objective value for instance $j$, which can be a lower bound or the best-known solution. As at each point $x$, each metaheuristic collects 25 samples for each instance, the final $y$-value in a point $x$ is the median of the instances' median sample. In these charts, the reference line $(y=1)$ represents the benchmark objective value $f\left(Z_{j}^{*}\right)$. In other words, the charts summarize the results for all instances and indicate, for each time point, how close or far the algorithm' median solutions are from the benchmark value, providing insights on the algorithm convergence rates.

\section{A.6.1 Results}

We tested a number of different values to calibrate parameters of TS, SA and LAS. The following values were tested:

- TS: tbTime $=(5,10,25,50,100,200,400,600)$ and stSize $=(5,10,25,50,100,300$, $600)$.

- SA: $T_{0}=(100,500,1000), \alpha=(0.99,0.9999)$, st Size $=(1,5,10,25,50,100,300,600)$ and $\varepsilon=0$.

- LAS: $l s$ Size $=(100,500,1000,5000,10000)$ and stSize $=(1,5,10,25,50,100,300,600)$.

We analyze the behavior presented by different combinations of the above parameter values. Figure 20 shows the TS configurations obtained. The results indicate that the TS is very sensitive to the size of the neighborhood explored (parameter stSize). Small and large values for st Size lead the TS to converge to poor quality solutions. An appropriate value is around 100. Also, the best values for the tabu aspiration criterion (parameter tbTime) were observed to be 
between 10 and 100 . These results suggest that the TS metaheuristic must be carefully calibrated concerning neighborhood sizes.

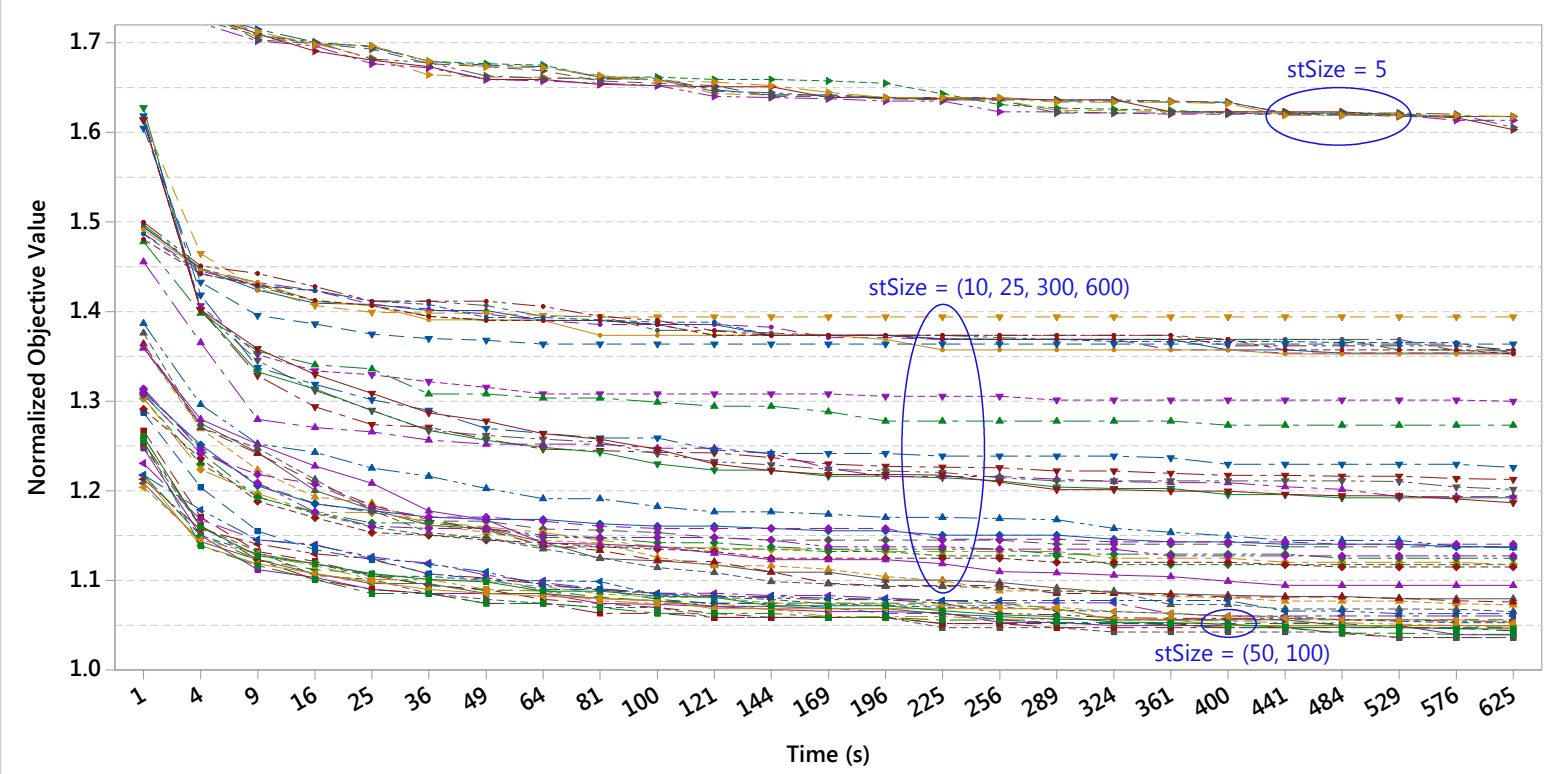

Figure 20 - Results of sequential TS for different parameter configurations.

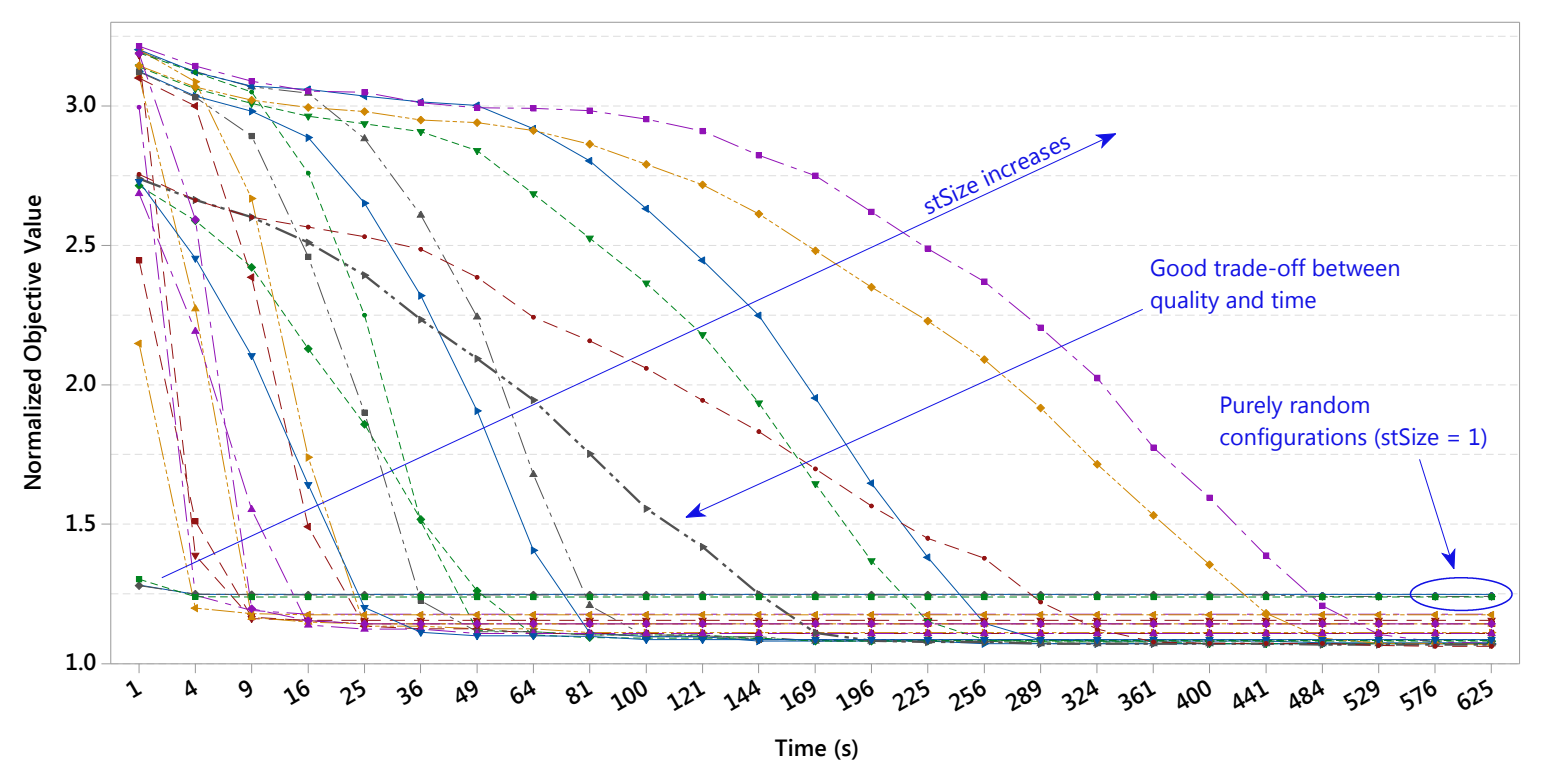

Figure 21 - Results of sequential SA for different parameter configurations.

Figure 21 shows the behavior of the SA for different configurations. The algorithm seems to be most sensitive to the cooling rate parameter. A good choice was $\alpha=0.9999$. We observed that even small negative perturbations in this value could worsen convergence rates. Also, we observed that when the SA tended to be purely random, with small values for parameter stSize, the SA converged to poor quality solutions. On the other hand, when stSize tended to large values, the SA converged to better solutions, although with a slower convergence rate. In Figure 
21 we plotted only configurations for $\alpha=0.9999$. Configurations for $\alpha=0.99$ performed as badly as purely random configurations.

Figure 22 shows the LAS configurations. The results show that LAS is not sensitive to the way in which the neighborhood is explored, parameter stSize. On the other hand, its convergence strongly depends on the length of the list of late accepted solutions, parameter $l s S i z e$. Large size lists lead the algorithm to converge to better quality solutions. However, the convergence is slow.

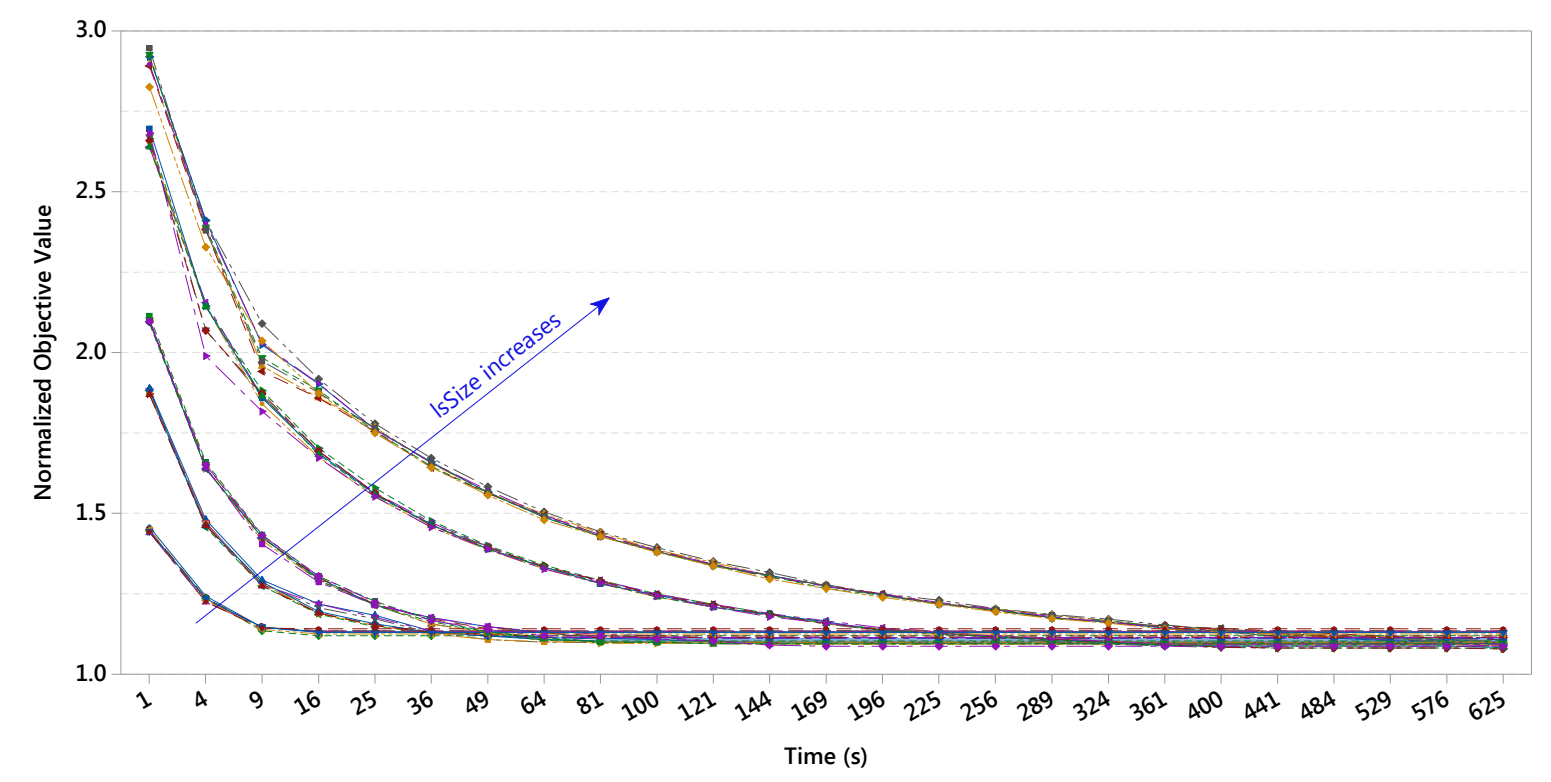

Figure 22 - Results of sequential LAS for different parameter configurations.

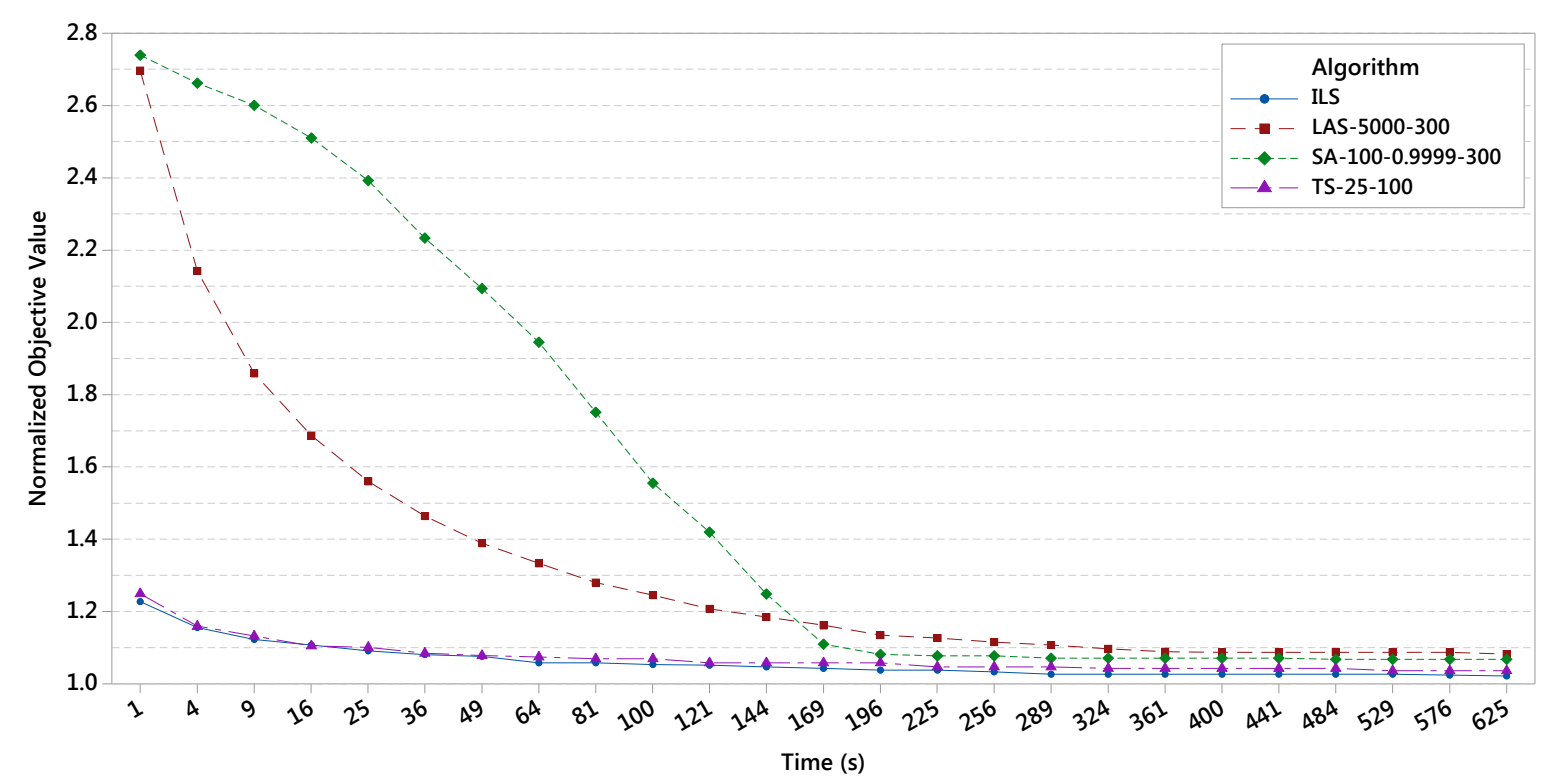

Figure 23 - Best configurations for sequential metaheuristics.

Figure 23 plots the best observed configurations for TS with $($ tbTime, stSize $)=(25,100)$, SA with $\left(T_{0}, \alpha\right.$, st Size $)=(100,0.9999,300)$ and LAS with $($ lsSize, stSize $)=(5000,300)$ together 
with the ILS results. The benchmark value for an instance $j$ is the objective value $f\left(Z_{j}^{*}\right)$ of the best solution $Z_{j}^{*}$ found in the experiments with these sequential algorithms. The chart in Figure 23 shows that ILS and TS presented better results than SA and LAS, in general. The median objective values of the four algorithms after 625 seconds of execution are 2.2\% (ILS), 3.6\% (TS), $6.8 \%$ (SA) and $8.3 \%$ (LAS) worse than the benchmark values. While SA and LAS take 625 seconds to reach solutions that are around 7 to $8 \%$ worse than the benchmark values, ILS and TS compute solutions with the same quality in less than 80 seconds. Also, we note that ILS perform slightly better in quality than TS. 

APPENDIX

THE IMPROVED RELAX-AND-FIX HEURISTIC 


\title{
A Parallel Multi-Start Iterated Local Search and a Proximity Relax-and-Fix heuristic for High School Timetabling Problem
}

\author{
Landir Saviniec $^{\star}$, Maristela Oliveira Santos ${ }^{\star}$, Alysson Machado Costa ${ }^{\dagger}$ \\ landir.savinieclgmail.com, marilicmc.usp.br, \\ alysson.costa@unimelb.edu.au \\ *Instituto de Ciências Matemáticas e de Computação - Universidade de São Paulo - Brasil \\ ${ }^{\dagger}$ School of Mathematics and Statistics - The University of Melbourne - Australia
}

\begin{abstract}
The high school timetabling problem has been frequently considered in the literature, but few studies employing parallel strategies have been proposed. In this paper, we propose a parallel cooperative multi-start approach. The method constructs an initial feasible solution via a Proximity Relax-and-Fix heuristic and applies a Parallel Multi-Start Iterated Local Search to further improve the initial solution. Computational experiments with practical instances show that our approach outperforms the state-of-the-art algorithms for variants of the problem. Our results clearly demonstrate the power of this type of approach to deal with complex combinatorial problems, such as the high school timetabling problem.
\end{abstract}

KEYWORDS. High School Timetabling Problem. Proximity Relax-and-Fix. Parallel MultiStart Iterated Local Search.

Paper topics: Parallel Algorithms in CPU \& GPU. Metaheuristics. Combinatorial Optimization. 


\section{Introduction}

The scientific literature has branched educational timetabling problems in three main families: University course timetabling [Lewis et al., 2007; Di Gaspero et al., 2007], high school timetabling [Pillay, 2014] and examination timetabling [McCollum et al., 2007]. Each category contains a rich number of specific constraints and particularities.

In this paper, we investigate parallel metaheuristics for the high school timetabling problem (HSTP). Due to its combinatorial characteristics, the HSTP resolution has been mainly based on approximations by sequential metaheuristics. According to our knowledge, only four studies have addressed the design of parallel metaheuristics for HSTP. Abramson [1991] proposed a Simulated Annealing which analyzes several simultaneous moves. Abramson and Abela [1992] proposed a Genetic Algorithm that evaluates several individuals of the population in parallel. Srndic et al. [2009] proposed a Genetic Algorithm which splits the global population into small islands that are managed in parallel. Saviniec et al. [2015] proposed an Iterated Local Search that, at each iteration, chooses the best local minimum among a set of local minima returned by multiples local search procedures running simultaneously.

Alba et al. [2013] reviewed the literature of parallel metaheuristics and identified a fast grown on these techniques in the last few years. The authors also classified the parallel frameworks and enumerated a few challenge topics that will be the main lines of research in the upcoming years.

According to Alba et al. [2013], parallel metaheuristics can be classified into populationbased and trajectory-based methods. Population-based metaheuristics are methods that keep a pool of solutions (e.g. Genetic Algorithms and Ant Colony Optimization). Trajectory-based metaheuristics are methods that keep a single current solution (e.g. Simulated Annealing and Tabu Search). These two categories can also, be classified by the type of framework implementation [Alba et al., 2013].

Population-based metaheuristics can be classified into i) Parallel individuals evaluationseveral individuals in the population are evaluated in parallel, and ii) Parallel islands - the population is split into subpopulations that can be managed in parallel.

Trajectory-based metaheuristics can be classified into i) Parallel moves - the neighboring solutions of the current solution are searched in parallel, ii) Move acceleration - the objective function of a single solution may be decomposed and evaluated in parallel, and iii) Parallel multistart - several asynchronous threads, cooperative or independent, run simultaneously to search different regions of the solution space.

Among the hot topics for future research in parallel metaheuristics, Alba et al. [2013] point out the design of parallel versions of well-known metaheuristics that could benefit from specific hardware architectures. In this paper, we propose a parallel multi-start approach based on Iterated Local Search that is suitable for multi-core machines. The algorithm is designed to exploit diversification, intensification, and cooperation among asynchronous threads during the search. We also propose a constructive heuristic based on Relax-and-Fix [Dillenberger et al., 1994] and Proximity Search [Fischetti and Monaci, 2014] to provide initial solutions to our parallel approach.

The remainder of the paper is organized as following. Section 2 describes the addressed problem by a mixed-integer programming formulation. Section 3 explains our parallel approach. Section 4 presents and discusses our computational experiments. The paper ends in Section 5 with some final remarks.

\section{The high school timetabling problem}

We focus on an HSTP motivated by rules of Brazilian high schools. In this context, the schools have a set of classes and a set of teachers. Classes are disjoint groups of students enrolled in the same set of subjects (e.g.: mathematics, physics and etc.). Each subject has a pre-assigned teacher and a number of meetings that must be scheduled to run during the week (e.g. Monday to Friday). The goal of the problem is to obtain a weekly timetable for these meetings. An input of the problem is described by the following definitions: 


\begin{tabular}{ll}
\hline Notation & Definition \\
\hline Sets & a set of classes. \\
$T$ & a set of teachers. \\
$D$ & a set of weekdays. \\
$H$ & a set of periods per day, which is equal to every day. \\
$H_{t d}$ & the set of periods for which teacher $t \in T$ is available during day $d \in D$. \\
$R$ & a set of subjects' requirements. A requirement specifies that a teacher $t \in T$ \\
& and a class $c \in C$ must meet a specified number of times during the week. \\
$R_{c}$ & the set of requirements that belongs to class $c \in C$. \\
$R_{t}$ & the set of requirements that belongs to teacher $t \in T$. \\
Parameters & \\
$\widetilde{\theta}_{r} \in \mathbb{N}$ & the number of weekly meetings specified in requirement $r \in R$. \\
$\widetilde{\delta}_{r} \in \mathbb{N}$ & a daily limit for lessons involving requirement $r \in R$. \\
$\widetilde{\pi}_{r} \in \mathbb{N}$ & a desired number of double lessons for requirement $r \in R$. Double lessons are \\
& two lessons in consecutive periods of the same day. \\
\hline
\end{tabular}

An output of the problem is represented by a set of binary variables $x_{r d h}$ which indicate whether the $r$-th requirement is scheduled to period $h \in H$ of day $d \in D$. A feasible solution is an assignment of values to variables $x_{r d h}$ that respects the following hard requirements:

1. Meeting of weekly required lessons: each requirement must be scheduled.

$$
\sum_{d \in D} \sum_{h \in H} x_{r d h}=\widetilde{\theta}_{r} \quad \forall r \in R
$$

2. No clashes in classes' schedules: each class must attend exactly one meeting per period.

$$
\sum_{r \in R_{c}} x_{r d h}=1 \quad \forall c \in C ; d \in D ; h \in H
$$

3. No clashes in teachers' schedules: each teacher must teach at most one lesson per period.

$$
\sum_{r \in R_{t}} x_{r d h} \leq 1 \quad \forall t \in T ; d \in D ; h \in H_{t d}
$$

4. No assignment of teachers in their unavailable periods: teachers must not be assigned to their unavailable periods.

$$
\sum_{r \in R_{t}} x_{r d h}=0 \quad \forall t \in T ; d \in D ; h \in H \backslash H_{t d}
$$

5. No daily workload violation for requirements: each requirement $r \in R$ must not be involved in more than $\widetilde{\delta}_{r}$ meetings per day.

$$
\sum_{h \in H} x_{r d h} \leq \widetilde{\delta}_{r} \quad \forall r \in R ; d \in D
$$

6. No holes in requirements' schedules: the schedule of each requirement must be consecutive within the same day.

$$
\begin{aligned}
& x_{r d i}-x_{r d h}+x_{r d j}-1 \leq 0 \quad \forall r \in R ; d \in D ; h=1, \cdots,|H|-2 ; \\
& i=0, \cdots, h-1 ; j=h+1, \cdots,|H|-1
\end{aligned}
$$

An optimal feasible solution minimizes the penalties associated with the following soft requirements:

7. Meeting of double lessons for requirements: for each requirement $r \in R$, the minimum number of $\widetilde{\pi}_{r}$ consecutive double lessons should be met. The auxiliary variables $\widehat{\phi}_{r d h}$ are set 
to 1 if a double lesson ends in periods $h=1, \cdots,|H|-1$ within each day, while variables $\widehat{\pi}_{r}$ quantify the number of unmet weekly double lessons.

$$
\begin{aligned}
\widehat{\phi}_{r d h} \leq x_{r d h} & \forall r \in R ; d \in D ; h=1, \cdots,|H|-1 \\
\widehat{\phi}_{r d h} \leq x_{r, d, h-1} & \forall r \in R ; d \in D ; h=1, \cdots,|H|-1 \\
\widehat{\phi}_{r d h} \leq 1-\widehat{\phi}_{r, d, h-1} & \forall r \in R ; d \in D ; h=2, \cdots,|H|-1 \\
\widehat{\pi}_{r} \geq \widetilde{\pi}_{r}-\sum_{d \in D} \sum_{h=1}^{|H|-1} \widehat{\phi}_{r d h} & \forall r \in R \\
\widehat{\phi}_{r d h} \geq 0 & \forall r \in R ; d \in D ; h=1, \cdots,|H|-1 \\
\widehat{\pi}_{r} \geq 0 & \forall r \in R
\end{aligned}
$$

8. No idle periods in teachers' schedules: teachers should not have idle periods in their daily schedules. A teacher $t$ is idle in periods $h=1, \cdots,|H|-2$ of a day $d$ if he/she is not busy, but is busy in earlier and later periods of the same day. Idles periods are flagged by the auxiliary variables $\widehat{j}_{t d h}$.

$$
\begin{aligned}
& \widehat{j}_{t d h} \geq \sum_{r \in R_{t}}\left(x_{r d i}-x_{r d h}+x_{r d j}\right)-1 \forall t \in T ; d \in D ; h=1, \cdots,|H|-2 ; \\
& i=0, \cdots, h-1 ; j=h+1, \cdots,|H|-1 \\
& \widehat{j}_{t d h} \geq 0 \quad \forall t \in T ; d \in D ; h=1, \cdots,|H|-2
\end{aligned}
$$

9. Compact schedules for teachers: for each teacher, the weekly schedule should encompass a minimum number of working days.

$$
\begin{gathered}
\widehat{d}_{t d} \geq \sum_{r \in R_{t}} x_{r d h} \quad \forall t \in T ; d \in D ; h \in H_{t d} \\
\widehat{d}_{t d} \geq 0 \quad \forall t \in T ; d \in D
\end{gathered}
$$

The complete problem can thus, be described by the following mixed-integer programming (MIP) formulation.

$$
\text { Minimize } f(x)=\sum_{r \in R} \alpha_{7} \widehat{\pi}_{r}+\sum_{t \in T} \sum_{d \in D} \alpha_{9} \widehat{d}_{t d}+\sum_{h=1}^{|H|-2} \alpha_{8} \widehat{j}_{t d h}
$$

Subject to:

(1) - (16)

$$
x_{r d h} \in\{0,1\} \quad \forall r \in R ; d \in D ; h \in H
$$

The objective function (17) minimizes the number of violations in requirements $i=$ $7, \cdots, 9$. The constant $\alpha_{i}$ is an associated penalty that express the importance of the $i$-th requirement.

This formulation can also be augmented by the cuts (20), proposed by Souza [2000], which specify that a teacher cannot work less than a minimum number of working days.

$$
\sum_{d \in D} \widehat{d}_{t d} \geq \max \left\{\left\lceil\frac{\sum_{r \in R_{t}} \widetilde{\theta}_{r}}{|H|}\right\rceil, \max _{r \in R_{t}}\left\{\left\lceil\frac{\widetilde{\theta}_{r}}{\widetilde{\delta}_{r}}\right\rceil\right\}\right\} \quad \forall t \in T
$$

\section{The proposed approach}

We propose an approach with constructive and improvement phases. The approach applies a heuristic based on Relax-and-Fix and Proximity Search (Section 3.1) to construct an initial feasible solution that is further improved by a Parallel Multi-Start Iterated Local Search (Section 3.2). 


\section{The constructive Proximity Relax-and-Fix heuristic (PRF)}

The original Relax-and-Fix (RF) [Dillenberger et al., 1994] is an MIP heuristic that constructs integer solutions by solving a series of relaxed sub-problems. Let's assume that $X$ is the set of integer variables of an MIP model. The RF heuristic relaxes the integrality of all variables of $X$ and splits them in $n$ subsets $X^{k}$, such that $X=\bigcup_{k=0}^{n-1} X^{k}$. At each iteration $k$, the variables belonging to subset $X^{k}$ are converted to integer and the resulting problem is solved. If it results in a feasible solution, then the variables of $X^{k}$ are fixed to their current values and the heuristic is repeated to iteration $k+1$. Otherwise, no solution is possible for the chosen partition and the heuristic stops. If the RF heuristic does not stop prematurely, it provides an integer solution at the end.

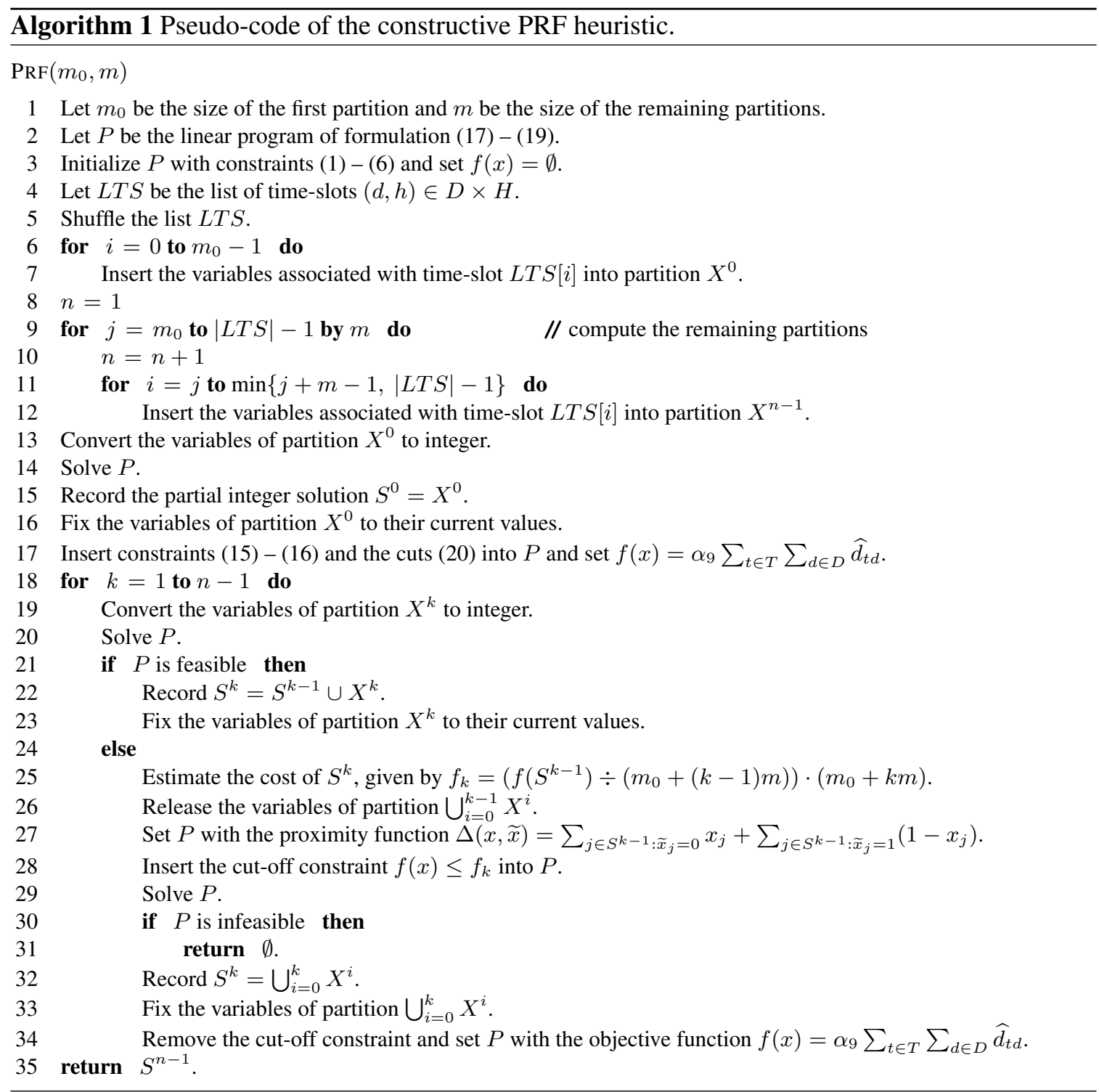

The drawback of the original RF heuristic, when applied to the HSTP described in Section 2 , is that the fixation of a partition $X^{k}$, at iteration $k$, very often leads to an infeasible problem at iteration $k+1$. To overcome this limitation, we propose a variant of the RF heuristic which solves a proximity problem [Fischetti and Monaci, 2014] to escape from these infeasible partitions. Silva [2013] proposed a similar strategy to deal with this limitation of the RF heuristic in lot-sizing problems. The author employs a local branch constraint instead of a proximity model to find new 
feasible partitions and escape from infeasibilities.

The proposed heuristic (PRF) is shown in Algorithm 1. The algorithm is a relax-andfix strategy with the addition of lines 25 to 34 , that solve the proximity problem to escape from infeasible partitions. At each iteration $k$, every time that the algorithm detects an infeasible partition, the line 25 estimates the cost $f_{k}$ of the partial solution $S^{k}$, based on the previous solution $S^{k-1}$. The line 26 releases all fixed variables and lines 27 to 29 set and solve the proximity problem. The proximity problem tries to find a partial solution $S^{k}$ that is not greater than $f_{k}$ and minimizes the changes in the values previously fixed for variables of $S^{k-1}$. If the proximity problem is feasible, a new partial solution $S^{k}$ is found, all variables in the partition $\bigcup_{i=0}^{k} X^{i}$ are fixed to their current values and the algorithm continues in the next iteration. Otherwise, it stops without any solution.

In our implementation, each partition contains the variables associated with a subset of time-slots $(d, h) \in D \times H$. We defined the first partition with size $m_{0}=10$ time-slots and the rest with $m=5$ time-slots. Also, every time that the program $P$ is solved in lines 14, 20 and 29, we stop it at the first solution found, to speed up the execution.

\section{The Parallel Multi-Start Iterated Local Search (PMILS)}

The proposed Parallel Multi-Start Iterated Local Search (PMILS), shown in Figure 1, employs a shared-memory manager/works strategy. The central idea of the PMILS is to exploit diversification/intensification and cooperation among threads. The algorithm has a manager thread (the diversifier agent) that shares information with $\mathrm{N}$ worker threads (the intensifier agents) via two communication buffers, "InputBuffer" and "OutputBuffer". All threads are Iterated Local Search [Lourenço et al., 2003] metaheuristics.

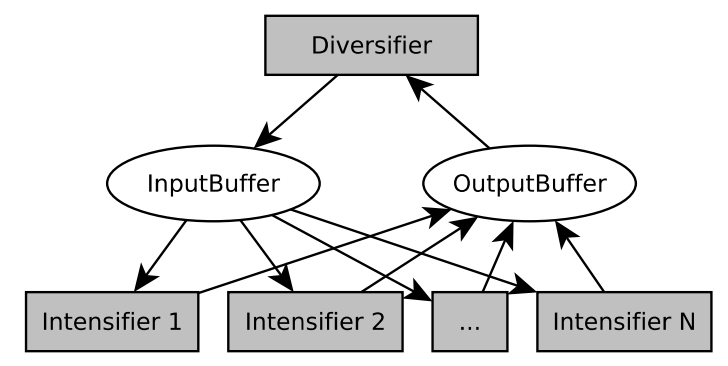

Figure 1: Diagram of communication among the agent threads of the PMILS algorithm.

The PMILS pseudo-code is shown in Algorithm 2. The diversifier agent starts at an initial solution and at each iteration, puts its local minimum into the input buffer (lines 10-14) to be further explored by the intensifier agents. The intensifiers get solutions from the input buffer (line 3 ) and intensify the search in their nearby neighborhoods (line 6). When an intensifier reaches its timelimit intTimeLim, it puts its best solution into the output buffer (line 7) to be analyzed by the diversifier. When the diversifier retrieves a solution $S_{r}$ from the output buffer (line 15), it checks for acceptance. If $S_{r}$ improves its best solution $S^{*}$, then the diversifier restarts its search from $S_{r}$ (line 19). Otherwise, $S_{r}$ is discarded.

The difference between the diversifier and the intensifier threads is that, while the diversifier walks through the solution space (lines 6-7) by only restarting from the global best solution when an improved solution is found (line 19). The intensifiers explore the nearby neighborhood of the local minima found by the diversifier, by always restarting from their private best solutions, see line 3 of the ILS procedure.

The timetable solutions used by the PMILS algorithm are represented by non-negative integer arrays $S$, where $S_{c d h}$ stores the teacher $t \in T$ assigned to teach to class $c \in C$ on period $h \in H$ of day $d \in D$. The neighborhood operator is the TQ operator proposed by Saviniec et al. [2013], which is based on the idea of Kempe Chain Interchanges [Lü et al., 2011] and explores moves by exchanging conflicting teachers between two time-slots $(d, h) \in T S$, with $T S=D \times$ 
$H$. The local search procedure is a first improvement strategy that explores all pairs of time-slots $(p, q) \in T S \times T S$, for $p \neq q$.

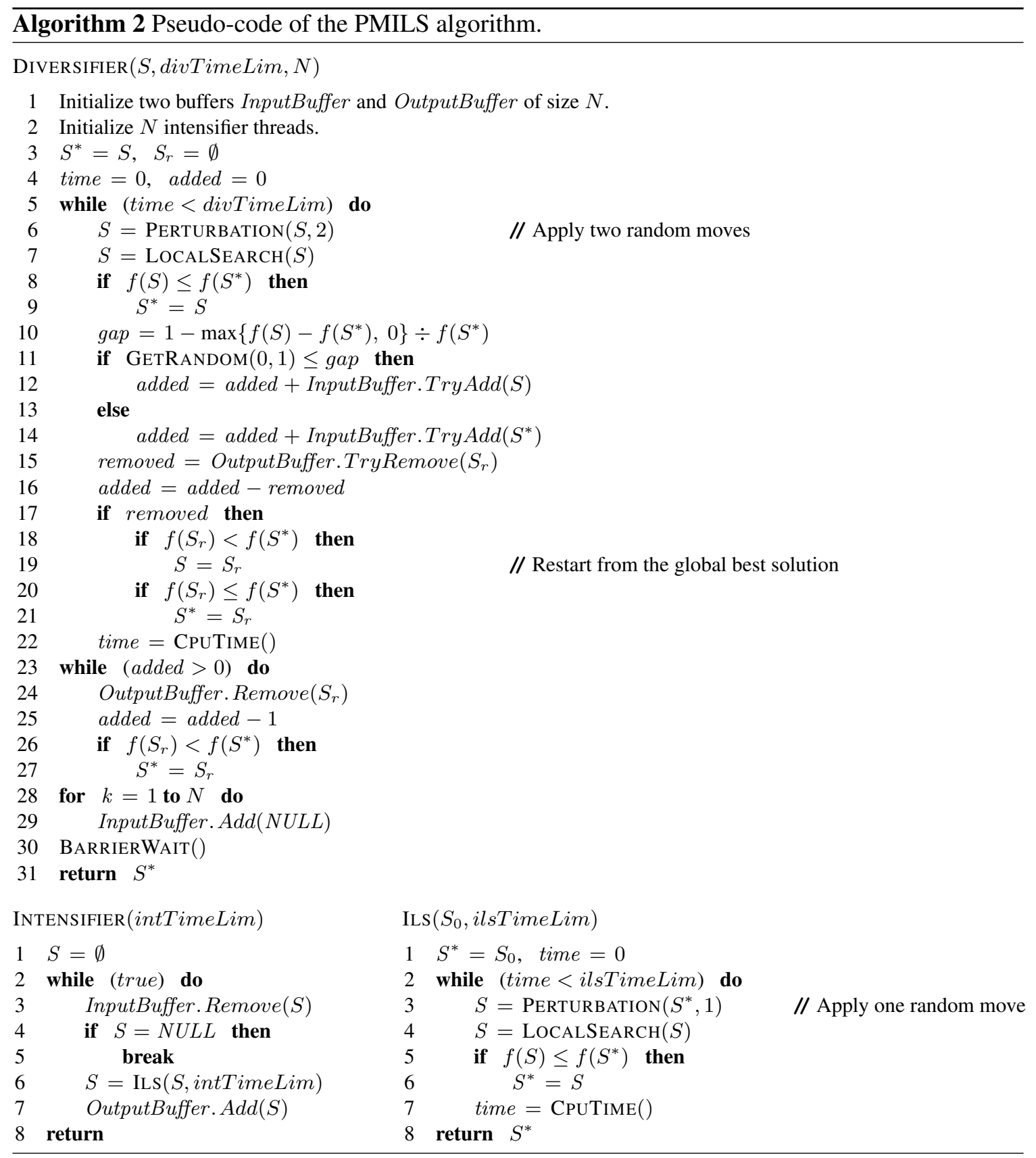

\section{Computational experiments}

We tested the proposed approach with two variants of the problem described in Section 2. The first variant HSTP-A is the problem introduced by Souza [2000], for which we show the results in Section 4.1. The second variant HSTP-B is a modification of the problem proposed by Souza [2000], for which we show the results in Section 4.2. The algorithms were coded in $\mathrm{C}++$ and compiled with GCC on Linux operating system. To implement parallelism, we employed the Pthreads library available in the GCC compiler. The MIP model and the PRF heuristic were implemented with callable libraries of CPLEX 12.6.

\section{Results in the HSTP-A}

This problem was introduced by Souza [2000]. The problem is a relaxation of the problem described in Section 2, for which hard constraints (6) are dropped. 
In this experiment, we tested the proposed approach in the set of seven instances ${ }^{1}$ proposed by Souza [2000]. The instances, shown in Table 1, have optimal solutions known, which is a contribution of several previous researches using heuristic solutions [Souza et al., 2003; Santos et al., 2005; Saviniec et al., 2013; Dorneles et al., 2014] and column generation lower bounds [Santos et al., 2012]. According to Santos et al. [2005], the objective function weighting parameters for these instances are: $\alpha_{7}=1, \alpha_{8}=3, \alpha_{9}=9$. For this experiment, we set the algorithm PMILS with parameters $N=3$ intensifiers, intTimeLim $=2$ seconds and total execution time (the two phases) of 625 seconds.

Table 1: Features of the instances proposed by Souza [2000].

\begin{tabular}{rrrrrrrr}
\hline ID & $|C|$ & $|T|$ & $|D|$ & $|H|$ & $\sum_{r \in R} \widetilde{\theta}_{r}$ & $\sum_{r \in R} \widetilde{\pi}_{r}$ & Optimal values \\
\hline 1 & 3 & 8 & 5 & 5 & 75 & 21 & 202 \\
2 & 6 & 14 & 5 & 5 & 150 & 29 & 333 \\
3 & 8 & 16 & 5 & 5 & 200 & 4 & 423 \\
4 & 12 & 23 & 5 & 5 & 300 & 41 & 652 \\
5 & 13 & 31 & 5 & 5 & 325 & 71 & 762 \\
6 & 14 & 30 & 5 & 5 & 350 & 63 & 756 \\
7 & 20 & 33 & 5 & 5 & 500 & 84 & 1017 \\
\hline
\end{tabular}

Table 2: Results of 25 runs of our approach compared to previous methods in the instances of Souza [2000].

\begin{tabular}{|c|c|c|c|c|c|c|c|c|c|}
\hline \multirow[b]{2}{*}{ ID } & \multicolumn{2}{|c|}{ PRF } & \multicolumn{3}{|c|}{ PMILS } & \multicolumn{2}{|c|}{ ILS } & \multicolumn{2}{|c|}{ F8 } \\
\hline & Time (s) & Median & Time (s) & Median & Best & Time (s) & Best & Time (s) & Best \\
\hline 1 & 0.12 & 358 & 7.00 & 202 & 202 & 900 & 202 & 600 & 202 \\
\hline 2 & 0.56 & 668 & 11.93 & 333 & 333 & 900 & 333 & 600 & 333 \\
\hline 3 & 0.77 & 700 & 529.01 & 426 & 423 & 900 & 423 & 21600 & 423 \\
\hline 4 & 6.04 & 824 & 19.00 & 652 & 652 & 900 & 652 & 600 & 652 \\
\hline 5 & 4.27 & 1345 & 9.87 & 762 & 762 & 900 & 762 & 600 & 762 \\
\hline 6 & 3.08 & 1425 & 13.80 & 756 & 756 & 900 & 756 & 1800 & 759 \\
\hline 7 & 13.29 & 1872 & 18.23 & 1017 & 1017 & 900 & 1017 & 1800 & 1017 \\
\hline
\end{tabular}

The results are shown in Table 2. The table compares the results of our approach with best results of two previous approaches. The Iterated Local Search (ILS) proposed by Saviniec et al. [2013] and the best version (F8) of the Fix-and-Optimize heuristics proposed by Dorneles et al. [2014]. The best solutions are shown in bold font. We observe that our PRF heuristic is very fast to construct initial solutions. The constructed solutions have an average optimality gap of 41.45 $\%$. The PMILS algorithm found similar results in quality of solutions compared with the two other approaches. However, PMILS outperforms the previous approaches in computational time. It finds the same quality of solutions much faster than the previous approaches ${ }^{2}$.

\section{Results in the HSTP-B}

This is the problem described in Section 2. In this problem, we used the 34 instances proposed in Saviniec et al. [2015]. The instances are described in Table 3. The objective function weighting parameters were set to $\alpha_{7}=1, \alpha_{8}=3, \alpha_{9}=9$.

In this experiment, our approach is compared with the solver GOAL [Fonseca et al., 2014], that is the winner of the Third International Timetabling Competition [Post et al., 2016] devoted to high school timetabling problems, ITC2011 ${ }^{3}$. We compare our approach with the latest version of GOAL [Fonseca et al., 2016], which employs a three-phase approach. The first phase constructs an initial solution via the KHE software libraries [Kingston, 2015]. The second phase

\footnotetext{
${ }^{1}$ Available at the website "http://labic.ic.uff.br/Instance/index.php?dir= SchoolTimetabling"

${ }^{2}$ Our approach was run in a machine with inferior hardware resources than the machines described by Saviniec et al. [2013] and Dorneles et al. [2014]. We used a Notebook with CPU Intel Core i3 (2.3 GHz) and 2 GB of RAM, running Linux Mint 17.1.

${ }^{3}$ https://www.utwente.nl/ctit/hstt/
} 
employs a parallel Variable Neighborhood Search (PVNS) metaheuristic and the third phase refines the output of PVNS with Fix-and-Optimize MIP heuristics. The experiment was made on a server with 2 CPU Intel Xeon E5-2680v2 (2.8 GHz) and 128 GB of RAM, running Red Hat Enterprise Linux 6.5. We set the algorithm PMILS with $N=19$ intensifiers and the parallel VNS of GOAL with 20 threads. The additional parameters of GOAL were set with recommended values (alg-timelimit $=62$, form-timelimit $=62$, initial-soln $=\mathrm{KHE}$, algorithm $=$ SVNS, formulation $=$ FIXOPT, formfixopt-nresources $=5$ and formfixopt-optinarow $=5$ ).

The results are shown in Table 4. The best solutions are shown in bold font. We observe that our approach outperforms GOAL in quality of solutions for most of the tested instances. The algorithm PMILS only performed worse than GOAL for instance 8. This instance has a large number of teachers' unavailable periods, which considerably reduces the feasible region and it is expected that the Fix-and-Optimize MIP heuristics implemented in GOAL perform better than our local search. The columns LB and UB of Table 4 show the results of CPLEX after a time-limit of 3 hours. The CPLEX was initialized with a solution found by running the PMILS algorithm during 625 seconds. The CPLEX was able to improve the solution of PMILS only for the restricted instance 8 . The last column of Table 4 presents the best gaps found for these instances.

In this experiment, the PRF heuristic was also able to construct initial feasible solutions very fast. The constructed solutions have an average optimality gap of $37.89 \%$.

Table 3: Features of the instances proposed by Saviniec et al. [2015].

\begin{tabular}{|c|c|c|c|c|c|c|c|}
\hline ID & Instance & $|C|$ & $|T|$ & $|D|$ & $|H|$ & $\sum_{r \in R} \widetilde{\theta}_{r}$ & $\sum_{r \in R} \tilde{\pi}_{r}$ \\
\hline 1 & CL-CEASD-2008-V-A & 12 & 27 & 5 & 5 & 300 & 132 \\
\hline 2 & CL-CEASD-2008-V-B & 12 & 27 & 5 & 5 & 300 & 132 \\
\hline 3 & CL-CECL-2011-M-A & 13 & 31 & 5 & 5 & 325 & 144 \\
\hline 4 & CL-CECL-2011-M-B & 13 & 31 & 5 & 5 & 325 & 143 \\
\hline 5 & CL-CECL-2011-N-A & 9 & 28 & 5 & 5 & 225 & 107 \\
\hline 6 & CL-CECL-2011-V-A & 14 & 29 & 5 & 5 & 350 & 164 \\
\hline 7 & CM-CECM-2011-M & 20 & 51 & 5 & 5 & 500 & 234 \\
\hline 8 & CM-CECM-2011-N & 8 & 30 & 5 & 5 & 200 & 96 \\
\hline 9 & CM-CECM-2011-V & 13 & 34 & 5 & 5 & 325 & 142 \\
\hline 10 & CM-CEDB-2010-N & 5 & 17 & 5 & 5 & 125 & 60 \\
\hline 11 & CM-CEUP-2008-V & 16 & 35 & 5 & 5 & 400 & 192 \\
\hline 12 & CM-CEUP-2011-M & 16 & 38 & 5 & 5 & 400 & 192 \\
\hline 13 & CM-CEUP-2011-N & 3 & 15 & 5 & 5 & 75 & 36 \\
\hline 14 & CM-CEUP-2011-V & 16 & 34 & 5 & 5 & 400 & 169 \\
\hline 15 & FA-EEF-2011-M & 4 & 12 & 5 & 5 & 100 & 42 \\
\hline 16 & JNS-CEDPII-2011-M & 8 & 19 & 5 & 5 & 200 & 85 \\
\hline 17 & JNS-CEDPII-2011-V & 7 & 21 & 5 & 5 & 175 & 73 \\
\hline 18 & JNS-CEJXXIII-2011-M & 5 & 18 & 5 & 5 & 125 & 60 \\
\hline 19 & JNS-CEJXXIII-2011-N & 4 & 15 & 5 & 5 & 100 & 48 \\
\hline 20 & JNS-CEJXXIII-2011-V & 5 & 18 & 5 & 5 & 125 & 60 \\
\hline 21 & MGA-CEDC-2011-M & 19 & 37 & 5 & 5 & 475 & 210 \\
\hline 22 & MGA-CEDC-2011-V & 12 & 31 & 5 & 5 & 300 & 131 \\
\hline 23 & MGA-CEGV-2011-M & 31 & 62 & 5 & 5 & 775 & 352 \\
\hline 24 & MGA-CEGV-2011-V & 32 & 75 & 5 & 5 & 800 & 357 \\
\hline 25 & MGA-CEJXXIII-2010-V & 16 & 35 & 5 & 5 & 400 & 192 \\
\hline 26 & MGA-CEVB-2011-M & 10 & 21 & 5 & 5 & 250 & 108 \\
\hline 27 & MGA-CEVB-2011-V & 9 & 20 & 5 & 5 & 225 & 97 \\
\hline 28 & NE-CESVP-2011-M-A & 18 & 45 & 5 & 5 & 450 & 212 \\
\hline 29 & NE-CESVP-2011-M-B & 18 & 44 & 5 & 5 & 450 & 212 \\
\hline 30 & NE-CESVP-2011-M-C & 18 & 45 & 5 & 5 & 450 & 211 \\
\hline 31 & NE-CESVP-2011-M-D & 18 & 45 & 5 & 5 & 450 & 211 \\
\hline 32 & NE-CESVP-2011-V-A & 16 & 44 & 5 & 5 & 400 & 183 \\
\hline 33 & NE-CESVP-2011-V-B & 16 & 43 & 5 & 5 & 400 & 184 \\
\hline 34 & NE-CESVP-2011-V-C & 16 & 43 & 5 & 5 & 400 & 182 \\
\hline
\end{tabular}


Table 4: Comparison between our approach and the solver GOAL [Fonseca et al., 2016] in 25 runs of 625 seconds.

\begin{tabular}{|c|c|c|c|c|c|c|c|c|c|}
\hline \multirow[b]{2}{*}{ ID } & \multicolumn{2}{|c|}{ PRF } & \multicolumn{2}{|c|}{ PMILS } & \multicolumn{2}{|c|}{ GOAL } & \multicolumn{2}{|c|}{ CPLEX (3 h) } & \multirow[b]{2}{*}{ Gap (\%) } \\
\hline & Time (s) & Median & Median & Best & Median & Best & LB & UB & \\
\hline 1 & 1.74 & 1158 & 710 & 703 & 730 & 716 & 682 & - & 2.99 \\
\hline 2 & 1.94 & 1146 & 713 & 706 & 730 & 716 & 682 & - & 3.40 \\
\hline 3 & 1.54 & 1514 & 751 & 746 & 770 & 762 & 726 & - & 2.68 \\
\hline 4 & 1.19 & 1583 & 743 & 738 & 768 & 749 & 725 & - & 1.76 \\
\hline 5 & 0.65 & 1253 & 631 & 631 & 648 & 642 & 629 & - & 0.32 \\
\hline 6 & 1.84 & 1521 & 777 & 773 & 814 & 800 & 771 & - & 0.26 \\
\hline 7 & 6.34 & 1583 & 1256 & 1245 & 1312 & 1297 & 1203 & - & 3.37 \\
\hline 8 & 1.53 & 805 & 697 & 678 & 682 & 676 & 675 & 675 & 0.00 \\
\hline 9 & 2.53 & 1002 & 822 & 816 & 838 & 829 & 804 & - & 1.47 \\
\hline 10 & 0.29 & 688 & 298 & 298 & 300 & 298 & 298 & - & 0.00 \\
\hline 11 & 3.64 & 1391 & 999 & 978 & 1052 & 1024 & 953 & - & 2.56 \\
\hline 12 & 5.39 & 1184 & 1037 & 1028 & 1048 & 1034 & 1014 & - & 1.36 \\
\hline 13 & 0.36 & 281 & 273 & 273 & 273 & 273 & 273 & - & 0.00 \\
\hline 14 & 4.64 & 1098 & 950 & 943 & 971 & 949 & 929 & - & 1.48 \\
\hline 15 & & & & Infeas & & & & & \\
\hline 16 & 0.61 & 870 & 483 & 481 & 498 & 490 & 475 & - & 1.25 \\
\hline 17 & 0.4 & 911 & 461 & 458 & 468 & 462 & 455 & - & 0.66 \\
\hline 18 & 0.31 & 762 & 319 & 319 & 324 & 319 & 316 & - & 0.94 \\
\hline 19 & 0.21 & 591 & 254 & 254 & 254 & 254 & 251 & - & 1.18 \\
\hline 20 & 0.3 & 784 & 325 & 325 & 328 & 325 & 312 & - & 4.00 \\
\hline 21 & 7.65 & 1266 & 1067 & 1061 & 1107 & 1091 & 1044 & - & 1.60 \\
\hline 22 & & & & Infeas & & & & & \\
\hline 23 & 11.8 & 2519 & 1893 & 1865 & 2013 & 1971 & 1793 & - & 3.86 \\
\hline 24 & 11.5 & 2665 & 2065 & 2047 & 2171 & 2128 & 1965 & - & 4.01 \\
\hline 25 & 4.02 & 1333 & 940 & 924 & 987 & 966 & 909 & - & 1.62 \\
\hline 26 & 1.34 & 860 & 575 & 572 & 603 & 584 & 570 & - & 0.35 \\
\hline 27 & 1.27 & 675 & 554 & 552 & 566 & 560 & 551 & - & 0.18 \\
\hline 28 & 2.63 & 2009 & 1153 & 1138 & 1183 & 1167 & 1103 & - & 3.08 \\
\hline 29 & 2.66 & 1958 & 1145 & 1133 & 1187 & 1168 & 1094 & - & 3.44 \\
\hline 30 & 2.82 & 2041 & 1166 & 1153 & 1203 & 1175 & 1112 & - & 3.56 \\
\hline 31 & 3.11 & 1853 & 1164 & 1147 & 1195 & 1181 & 1111 & - & 3.14 \\
\hline 32 & 1.97 & 1928 & 1043 & 1033 & 1069 & 1063 & 998 & - & 3.39 \\
\hline 33 & 2.12 & 1901 & 1050 & 1038 & 1078 & 1060 & 997 & - & 3.95 \\
\hline 34 & 2.32 & 1811 & 1041 & 1029 & 1070 & 1056 & 979 & - & 4.86 \\
\hline
\end{tabular}

\section{Conclusions}

In this paper, we proposed an approach with constructive and improvement phases to solve high school timetabling problems. The approach applies a Proximity Relax-and-Fix heuristic to construct an initial feasible solution that is further improved with a Parallel Multi-Start Iterated Local Search. Our constructive heuristic solves a series of relaxed sub-problems to achieve a completely feasible solution. When infeasible partitions are chosen, the heuristic is able to step over by solving a proximity problem to find another feasible partition. Our improvement heuristic is designed to exploit the parallelism of multi-core machines. The algorithm is a system of cooperative threads that exploit diversification and intensification to achieve high-quality solutions during the search. The proposed approach showed to find good quality solutions and outperform the stateof-the-art algorithms for two variants of the problem at hand. These results show that parallel cooperative multi-start approaches, such the one proposed here, are promising tools for handling combinatorial problems such as the HSTP. Also, these approaches are scalable algorithms that can exploit the power of machines with a large number of cores.

\section{Acknowledgments}

This research was supported by FAPESP-Brazil. Grants: 2013/13563-3 and 2015/100322. We would like to thank professor George H. G. Fonseca for sharing the code of GOAL. 


\section{References}

Abramson, D. (1991). Constructing school timetables using simulated annealing: sequential and parallel algorithms. Management science, 37(1):98-113.

Abramson, D. and Abela, J. (1992). A parallel genetic algorithm for solving the school timetabling problem. Proceedings of the 15 Australian Computer Science Conference, Hobart, Australia, p. $1-11$.

Alba, E., Luque, G., and Nesmachnow, S. (2013). Parallel metaheuristics: recent advances and new trends. International Transactions in Operational Research, 20(1):1-48.

Di Gaspero, L., McCollum, B., and Schaerf, A. (2007). The second international timetabling competition (itc-2007): Curriculum-based course timetabling (track 3). Technical report, Technical Report QUB/IEEE/Tech/ITC2007/CurriculumCTT/v1. 0, Queen's University, Belfast, United Kingdom.

Dillenberger, C., Escudero, L. F., Wollensak, A., and Zhang, W. (1994). On practical resource allocation for production planning and scheduling with period overlapping setups. European Journal of Operational Research, 75(2):275 - 286. ISSN 0377-2217.

Dorneles, Á. P., de Araújo, O. C., and Buriol, L. S. (2014). A fix-and-optimize heuristic for the high school timetabling problem. Computers \& Operations Research, 52, Part A(0):29- 38 .

Fischetti, M. and Monaci, M. (2014). Proximity search for 0-1 mixed-integer convex programming. Journal of Heuristics, 20(6):709-731. ISSN 1572-9397.

Fonseca, G. H., Santos, H. G., and Carrano, E. G. (2016). Integrating matheuristics and metaheuristics for timetabling. Computers \& Operations Research, 74:108 - 117.

Fonseca, G. H., Santos, H. G., Toffolo, T. A., Brito, S. S., and Souza, M. J. (2014). Goal solver: a hybrid local search based solver for high school timetabling. Annals of Operations Research, $\mathrm{p}$. 1-21. ISSN 0254-5330.

Kingston, J. H. (2015). A software library for high school timetabling. URL http: / / sydney . edu.au/engineering/it/ jeff/khe/.

Lewis, R., Paechter, B., and McCollum, B. (2007). Post enrolment based course timetabling: A description of the problem model used for track two of the second international timetabling competition. Cardiff Business School.

Lourenço, H., Martin, O., and Stützle, T. (2003). Iterated local search. Handbook of metaheuristics, p. $320-353$.

Lü, Z., Hao, J., and Glover, F. (2011). Neighborhood analysis: a case study on curriculum-based course timetabling. Journal of Heuristics, 17(2):97-118.

McCollum, B., McMullan, P., Burke, E. K., Parkes, A. J., and Qu, R. (2007). The second international timetabling competition: Examination timetabling track. Technical report, Technical Report QUB/IEEE/Tech/ITC2007/-Exam/v4.0/17, Queen's University, Belfast, United Kingdom.

Pillay, N. (2014). A survey of school timetabling research. Annals of Operations Research, 218(1): 261-293. ISSN 1572-9338.

Post, G., Di Gaspero, L., Kingston, J. H., McCollum, B., and Schaerf, A. (2016). The third international timetabling competition. Annals of Operations Research, 239(1):69-75. 
Santos, H., Ochi, L., and Souza, M. (2005). A tabu search heuristic with efficient diversification strategies for the class/teacher timetabling problem. Journal of Experimental Algorithmics, 10: $2-9$.

Santos, H., Uchoa, E., Ochi, L., and Maculan, N. (2012). Strong bounds with cut and column generation for class-teacher timetabling. Annals of Operations Research, 194(1):399-412.

Saviniec, L., Constantino, A. A., Romão, W., and Santos, H. G. (2013). Solving the high school timetabling problem to optimality by using ils algorithms. Proceedings of the Brazilian Symposium on Operations Research, Sobrapo, Rio de Janeiro, Brazil, p. 3330-3341.

Saviniec, L., Santos, M. O., Costa, A. M., and Constantino, A. A. (2015). Multithreading iterated local search aplicado ao problema de horários escolares. Proceedings of the Brazilian Symposium on Operations Research, Sobrapo, Rio de Janeiro, Brazil, p. 826-837.

Silva, D. H. (2013). Métodos híbridos para o problema de dimensionamento de lotes com múltiplas plantas. Master's thesis, Programa de Pós-Graduação em Ciências de Computação e Matemática Computacional - Instituto de Ciências Matemáticas e de Computação - Universidade de São Paulo, São Carlos.

Souza, M. J. F. (2000). Programação de Horários em Escolas: Uma Aproximação por Metaheurísticas. $\mathrm{PhD}$ thesis, Programa de Pós-Graduação em Engenharia de Sistemas e Computação - Universidade Federal do Rio de Janeiro, Rio de Janeiro.

Souza, M., Ochi, L., and Maculan, N. (2003). A grasp-tabu search algorithm for solving school timetabling problems. Metaheuristics: Computer Decision-Making. Kluwer Academic Publishers, Boston, p. 659-672.

Srndic, N., Pandzo, E., Dervisevic, M., and Konjicija, S. (2009). The application of a parallel genetic algorithm to timetabling of elementary school classes: A coarse grained approach. In Information, Communication and Automation Technologies, 2009. ICAT 2009. XXII International Symposium on, p. 1-5. IEEE. 


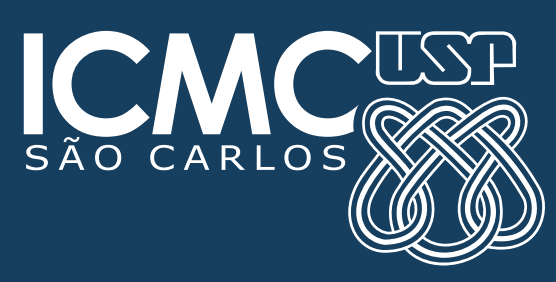

